\title{
ORGANIZATION AND MAINTENANCE OF MOLECULAR DOMAINS IN MYELINATED AXONS
}

\author{
Elizabeth Dunham Buttermore
}

A dissertation submitted to the faculty of the University of North Carolina at Chapel Hill in partial fulfillment of the requirements for the degree of Doctor of Philosophy in the Curriculum in Neurobiology

Chapel Hill

2012

Approved by:

Manzoor A. Bhat, $\mathrm{PhD}$

Glenn K. Matsushima, PhD

Ken D. McCarthy, PhD

Larysa H. Pevny, PhD

Benjamin D. Philpot, PhD 
(C)2012

Elizabeth Dunham Buttermore

ALL RIGHTS RESERVED 


\begin{abstract}
ELIZABETH DUNHAM BUTTERMORE: Organization and Maintenance of Molecular Domains in Myelinated Axons

(Under the direction of Manzoor A. Bhat)
\end{abstract}

Myelinated axons are segregated into molecular domains that are essential for action potential propagation. Each domain is characterized by clustering of specific molecular components and disruption of these domains, as seen in multiple sclerosis and ataxias, results in altered neuronal function. However, mechanisms responsible for domain organization remain elusive. We utilized mouse conditional knockouts to uncover mechanisms responsible for the organization and maintenance of the paranode, juxtaparanode, and axon initial segment (AIS).

Paranode disruption results in mislocalization of juxtaparanodal proteins and disorganization of the axonal cytoskeleton. Caspr and Caspr2, which localize to the paranode and juxtaparanode, contain binding sites for the cytoskeletal adaptor protein 4.1B. We generated $4.1 \mathrm{~B}$ null mice and showed that loss of $4.1 \mathrm{~B}$ resulted in severe disorganization of the juxtaparanode in both the peripheral (PNS) and central nervous system (CNS). At P30 PNS paranodes, loss of 4.1B disrupted Caspr localization and AGSJs. Loss of 4.1B at CNS paranodes resulted in progressive disruption of Caspr and AGSJs. Thus, 4.1B plays a role in interactions between the paranodal AGSJs and axonal cytoskeleton and is required for long-term maintenance of axonal domains. 
The cerebellar Purkinje AIS is targeted by basket axon collaterals that form the pinceau, which is critical for cerebellar function. Mechanistic details of pinceau organization are poorly understood. Loss of cytoskeletal adaptor protein AnkyrinG results in mislocalization of the cell adhesion molecule Neurofascin (Nfasc) at the Purkinje AIS and abnormal organization of the pinceau. We generated cell type-specific Nfasc null mice and found that Purkinje Nfasc is required for AIS maturation and for maintaining stable contacts between basket axon terminals and the Purkinje AIS during pinceau organization, while basket neuron Nfasc is required for proper basket axon collateral outgrowth and targeting to Purkinje soma/AIS. Disruption of the AIS and pinceau from loss of Nfasc leads to Purkinje neuron degeneration and ataxia. Together, the results presented in this dissertation elucidate mechanisms responsible for organization of the paranode, juxtaparanode, AIS, and pinceau. This knowledge will be critical for designing future therapeutic strategies to treat pathologies where restoration of axonal domains will be required to restore neuronal function. 


\section{DEDICATION}

I would like to dedicate this dissertation to my family, who has been there for me through every high and low of this journey.

To my husband, Nate Buttermore, who taught me to not take life too seriously and has been my best friend, shoulder to cry on, and biggest cheerleader of every accomplishment; not to mention my cook, maid and chauffeur throughout the busiest times of these past few years.

To my mom, Barb Dunham, who has been there for me to call on my bus ride home just about every day for the past 6 years. She has always been a voice of reason, support, and unconditional love that I will never be able to say thank you enough for.

To my dad, Roy Dunham, who helped my scientific career from before I even knew it was my direction in life. Countless hours were spent on science projects, college applications, and schoolwork along the way; thank you.

To my sister, Emily Jordan, who had confidence in my dreams before I even did and whose compassion for others is evident in everything she does.

To my in-laws, Megan, Alun, Elissa and Aaron Buttermore, thank you for allowing me to become a part of your family and for your loving encouragement and generosity along the way. 


\section{ACKNOWLEDGEMENTS}

The work presented in this dissertation would not have been possible without the support and guidance of my colleagues and friends at UNC Chapel Hill. First, I would like to thank my advisor, Manzoor Bhat, for always providing the resources and advice needed to keep my projects moving forward. I would also like to thank my committee members, Glenn Matsushima, Ken McCarthy, Larysa Pevny and Ben Philpot for their encouragement and input towards the completion of this work. I also thank the members of the Bhat lab, both past and present, including Swati Banerjee, Kevin Blauth, Yu-Chi Chen, Liz Fisher, James Green, Rosa Mino, Raehum Paik, Anil Pillai, and Courtney Thaxton for the unquantifiable time and support they have given to me during lab meetings, practice presentations and countless questions I have asked them. I am also grateful to Alan Fanning for helpful insight along the way and Bonnie Taylor-Blake for assistance with cryo-sectioning and management of $5^{\text {th }}$ floor NRB. I would also like to thank the Curriculum in Neurobiology, including Denise Kenney, Bob Rosenberg, Aldo Rustioni, and Bill Snider for helping to organize the framework and path that allowed me to reach this point in my career.

Parts of this work were done in collaboration with Ben Philpot at UNC Chapel Hill and Christian Hansel at the University of Chicago. I also would like to specifically thank Mike Wallace, the graduate student in Ben's lab who helped us with the biocytin injection experiments, and Claire Piochon, the postdoc in Christian's lab who performed 
the Purkinje neuron electrophysiological recordings. They each also contributed to the writing and editing of the results sections that came from their efforts.

I also want to thank the many core facility members who have helped me along the way. Specifically, Vicky Madden in the Microscopy Services Laboratory, Vladimir Ghukasyan in the Neuroscience Confocal and Multiphoton Imaging Facility, and Sheryl Moy and Randy Nonneman in the Mouse Behavioral Phenotyping Laboratory.

Finally, I would like to thank all my friends, whose support, advice and constant encouragement have helped me achieve my goals while making my life all the more interesting. 


\section{PREFACE}

Portions of this dissertation have been published or are in preparation to be published. Thank you to all the co-authors and those who contributed reagents and time to these publications:

Chapter 1 is a slightly revised version of Buttermore, E.D. and Bhat, M.A. (2012). Organization and Maintenance of Molecular Domains in Myelinated Axons. Manuscript in preparation.

Chapter 2 is a slightly revised version of Buttermore, E.D, Dupree, J.L., Cheng, J., An, X., Tessarollo, L., and Bhat, M.A. (2011). The cytoskeletal adaptor protein band 4.1B is required for the maintenance of paranodal axo-glial septate junctions in myelinated axons. Journal of Neuroscience. 31(22): 8013-8024. Permission to include this article in its entirety in my $\mathrm{PhD}$ dissertation was obtained from The Journal of Neuroscience as explained at http://www.jneurosci.org/site/misc/about.xhtml\#permission.

Chapter 3 is a revised version of Buttermore, E.D., Piochon, C., Wallace, M., Philpot, B., Hansel, C., and Bhat, M.A. (2012). Pinceau organization in the cerebellum requires distinct functions of Neurofascin in Purkinje and basket neurons during postnatal development. Journal of Neuroscience. 32(14) 4724-4742. Permission to include this article in its entirety in my $\mathrm{PhD}$ dissertation was obtained from The Journal of Neuroscience as explained at http://www.jneurosci.org/site/misc/about.xhtml\#permission. 


\section{TABLE OF CONTENTS}

List of Tables...........................................................

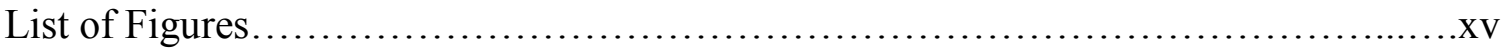

List of Abbreviations...............................................................

Chapters:

1. Introduction: Organization and maintenance of molecular domains in myelinated axons................................................. 1

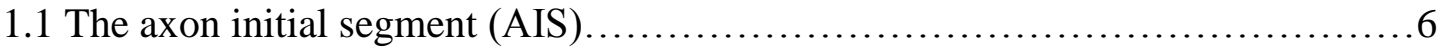

1.1.1 Formation of the AIS .............................................

1.1.2 The AIS is a barrier for axonal transport.......................... 10

1.1.3 Functional maturation of the AIS .................................11

1.1.4 Plasticity of the AIS .......................................... 12

1.1.5 Regulation of AIS function.................................... 13

1.1.6 AIS in disease and injury.................................... 17

1.2 The node of Ranvier.................................................. 19

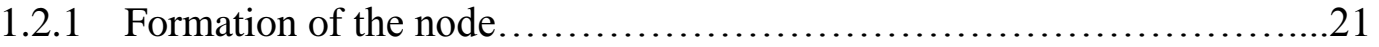

1.2.2 The node is a barrier against invading paranodes.....................25

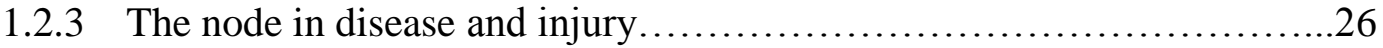

1.3 The paranode........................................................27

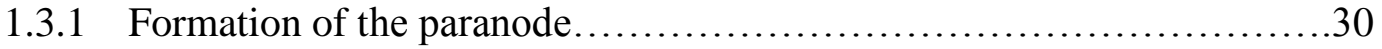


1.3.2 Role of axonal cytoskeleton in axo-glial septate junction (AGSJ) organization.

1.3.3 Role of lipid rafts in AGSJ organization.................................

1.3.4 The paranode is a fence against the invading juxtaparanodal complex....32

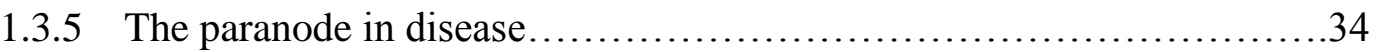

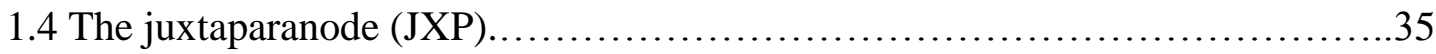

1.4.1 Role of axonal cytoskeleton in JXP organization......................36

1.4.2 Role of JXP potassium channels in action potential propagation...........37

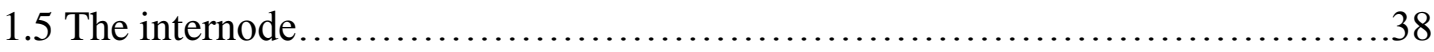

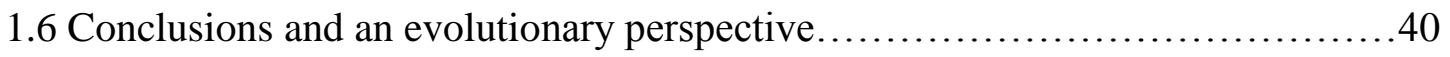

2. The cytoskeletal adaptor protein band $4.1 B$ is required for the maintenance of paranodal axo-glial septate junctions in myelinated axons .............43

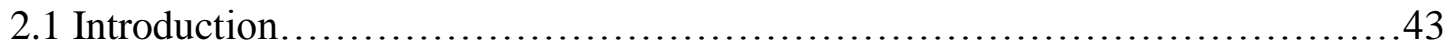

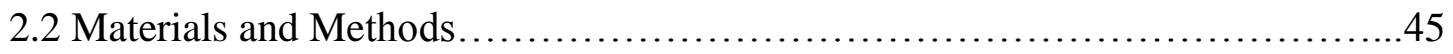

2.2.1 Animals..........................................................

2.2.2 Generation of 4.1B/Epb4.1l3 conditional mutants......................45

2.2.3 Generation of 4.1B antibodies.......................................46

2.2.4 Tissue preparation, antibodies and immunostaining.....................46

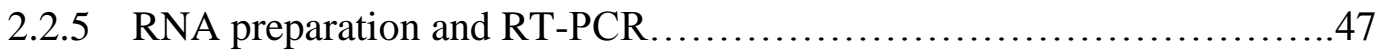

2.2.6 Immunoblotting and immunoprecipitation............................48

2.2.7 Transmission Electron Microscopy (TEM) ...........................49

2.2.8 Image analysis................................................... 49

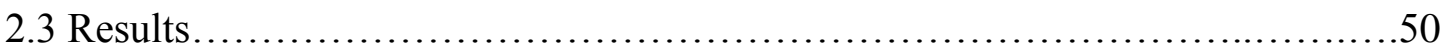

2.3.1 Developmental expression of 4.1B and generation of $4.1 B$ null mutants....................................................... 50 
2.3.2 4.1B is required for the organization and stabilization of the paranodal and juxtaparanodal regions in myelinated axons.

2.3.3 Molecular interactions between paranodal Caspr and complex formation between juxtaparanodal Caspr2, $\mathrm{K}_{\mathrm{V}} 1.2$ and 4.1B.

2.3.4 4.1B is critical for the stability of the paranodal axo-glial septate junctions

2.3.5 4.1R is not expressed at the PNS paranodes or enriched in $4.1 B^{-/-}$mutants

2.4 Discussion

2.4.1 Isoform specific functions of 4.1B in PNS and CNS myelinated axons................................................. 77

2.4.2 Organization of axonal domains and localization of 4.1B..............79

2.4.3 Role of axonal cytoskeleton in stabilizing axon-glial interactions and septate junctions.

2.4.4 Evolutionary role of cytoskeletal proteins in stabilizing axo-glial septate junctions

3. Pinceau organization in the cerebellum requires distinct functions of Neurofascin in Purkinje and basket neurons during postnatal development.....................83

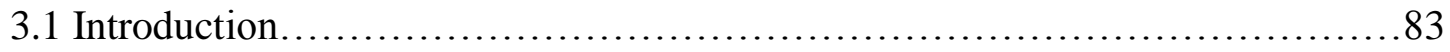

3.2 Materials and Methods...................................................... 85

3.2.1 Animals..................................................... 85

3.2.2 Tissue specific deletion of the $N f a s c$ gene........................... 85

3.2.3 Antibodies.................................................... 86

3.2.4 Tissue preparation and immunostaining............................86

3.2.5 Quantification and analysis................................... 87

3.2.6 Transmission Electron Microscopy (TEM) ..........................89

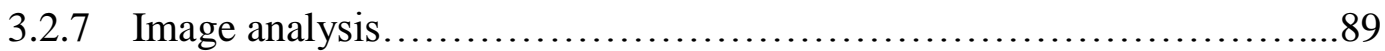




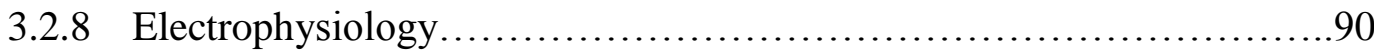

3.2.9 Behavioral testing ............................................... 92

3.2.10 Basket cell dye-injections......................................92

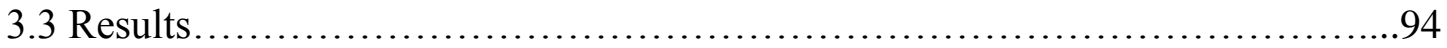

3.3.1 Developmental organization of the cerebellar pinceau...................94

3.3.2 Nfasc is expressed in cerebellar basket axon terminals

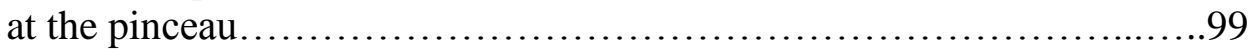

3.3.3 Nfasc is required for the maturation and maintenance of the Purkinje axon initial segment.........................................105

3.3.4 Nfasc is required in both basket neurons and Purkinje neurons for the organization of the pinceau.................................110

3.3.5 Nfasc is required in basket neurons for proper axon collateral outgrowth and targeting during pinceau formation....................118

3.3.6 Nfasc is required for proper basket axon collateral targeting and compaction at the pinceau.....................................123

3.3.7 Nfasc-deficient Purkinje neurons lack spontaneous activity and receive reduced inhibitory input from basket neurons.

3.3.8 Loss of Nfasc in Purkinje and basket neurons leads to progressive ataxia and neurodegeneration............................133

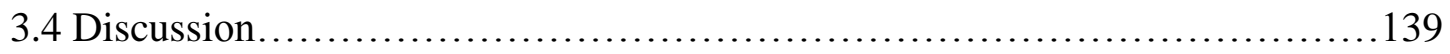

3.4.1 Neurofascin in adhesive interactions between Purkinje and basket neurons.

3.4.2 Loss of Nfasc in basket and/or Purkinje neurons and consequences on pinceau organization........................................... 141

3.4.3 Basket neuron inhibitory input to Purkinje neurons and the role of pinceau...................................................

3.4.4 Ataxia and neurodegeneration in Nfasc mutants.......................143

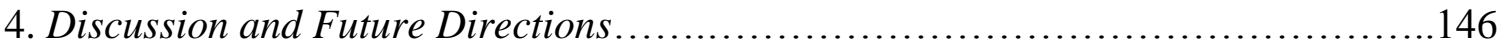


4.1 Axo-glial septate junctions link myelinating glia to the axonal cytoskeleton....147

4.2 Future directions for elucidating new paranodal proteins important for AGSJ stabilization............................................ 150

4.3 Different mechanisms are responsible for AIS maturation versus initial formation................................................... 153

4.4 Future directions for elucidating the primary organizer of the AIS..............154

4.5 Nfasc is required in both Purkinje and basket neurons for pinceau

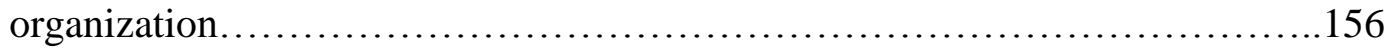

4.6 The pinceau modulates Purkinje neuron function......................... 158

4.7 Purkinje neuron degeneration, ataxia, and cerebellar disease.................160

4.8 Future directions for elucidating Nfasc binding partners and the mechanistic role for Nfasc in basket axon outgrowth........................163

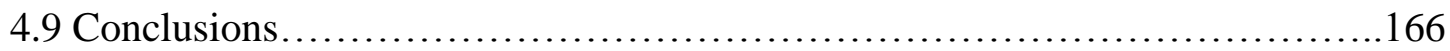

References............................................................ 168 


\section{List of Tables}

Table 1.1 Molecular components of molecular domains in myelinated axons.........4

Table 2.1 Phenotype differences between two independently generated

$4.1 B$ mutant alleles...............................................58 


\section{List of Figures}

Figure 1.1 Molecular domains in myelinated axons..............................

Figure 1.2 Molecular organization and maturation of the AIS .................... 8

Figure $1.3 \quad$ Organization of the cerebellar pinceau.............................. 16

Figure 1.4 Molecular components of the PNS node...........................20

Figure 1.5 Molecular organization of the paranode and AGSJs...................29

Figure $1.6 \quad$ Molecular organization of the JXP....................................

Figure 2.1 Primary Structure and Subcellular Localization of 4.1B and Generation of $4.1 B^{\text {Flox }}$ Mice..................................53

Figure 2.2 Loss of 4.1B Disrupts Paranodal and Juxtaparanodal Organization in the PNS Myelinated Axons............................57

Figure 2.3 Mislocalization of Juxtaparanodal Components and Destabilization of the Paranodes in the CNS Myelinated Axons of 4.1B Mutants.

Figure 2.4 Multiple 4.1B Isoforms in Sciatic Nerves and Molecular Interactions between 4.1B, Caspr and Caspr2. 63

Figure 2.5 4.1B is Required for the Stability of Paranodal Axo-glial Septate Junctions in Sciatic Nerve Myelinated Axons..................66

Figure 2.6 4.1B is Required for the Stability of Paranodal Axo-glial Septate Junctions in the Spinal Cord Myelinated Axons..................70

Figure 2.7 4.1R is not Enriched at Sciatic Nerve Paranodes in 4.1B Mutants.........74

Figure 2.8 Paranodal and juxtaparanodal proteins are not disrupted in the $\beta$-Actin-Cre; $4.1 B^{\text {Flox }}$ background strains.

Figure 2.9 Enrichment of 4.1B at the paranodal and juxtaparanodal domains is severely affected in Caspr and Cgt Mutants...

Figure 3.1 Molecular organization of the cerebellar pinceau during postnatal development. ...

Figure 3.2 Ubiquitous Ablation of Nfasc 100 
Figure 3.3 Purkinje and basket-specific ablation of Neurofascin reveals its localization to both Purkinje and basket neurons...................103

Figure 3.4 Early postnatal loss of Nfasc disrupts Purkinje AIS maturation and stabilization........................................ 108

Figure 3.5 Ablation of Nfasc disrupts basket axon targeting and pinceau organization.............................................. 114

Figure 3.6 Pinceau formation is not affected in Brevican and $\mathrm{NrCAM}$ mutants......117

Figure 3.7 Loss of Nfasc in basket neurons causes mistargeting of basket axon collaterals...

Figure 3.8 Cerebellar pinceau disorganization and infiltration of glial processes in Nfasc-deficient Purkinje and basket neuron cerebella

Figure 3.9 Purkinje neuron-specific ablation of Nfasc abolishes Purkinje neuron spontaneous activity and reduces inhibitory inputs to Purkinje neurons

Figure 3.10 Parallel Fiber (PF) inputs are unchanged in Purkinje neuronspecific Nfasc mutants

Figure 3.11 Purkinje neuron-specific loss of Nfasc results in ataxia.... 134

Figure 3.12 Ablation of Nfasc in Purkinje neurons leads to progressive Purkinje neuron degeneration 


\section{List of Abbreviations}

\begin{tabular}{|c|c|}
\hline $4.1 \mathrm{~B}$ & 4.1 brain \\
\hline $4.1 B^{\text {Flox }}$ & $4.1 B$ floxed allele for conditional $4.1 B$ ablation \\
\hline $4.1 \mathrm{G}$ & 4.1 global \\
\hline $4.1 \mathrm{~N}$ & 4.1 neuronal \\
\hline $4.1 \mathrm{R}$ & 4.1 erythrocyte \\
\hline ACSF & artificial cerebral spinal fluid \\
\hline AGSJs & axo-glial septate junctions \\
\hline AIS & axon initial segment \\
\hline ALS & amyotrophic lateral sclerosis \\
\hline AMPA & 2-amino-3-(5-methyl-3-oxo-1,2- oxazol-4-yl) propionic acid \\
\hline Ank/ANK & Ankyrin \\
\hline ANOVA & analysis of variance \\
\hline APV & ( $2 R$ )-amino-5-phosphonovaleric acid, NMDA receptor antagonist \\
\hline BAC & bacterial artificial chromosome \\
\hline$\beta$-Act-Cre & $\beta$-Actin-Cre \\
\hline Bcan & Brevican \\
\hline Calb & Calbindin \\
\hline CAM & cell adhesion molecule \\
\hline Caspr/CASP & Contactin-associated protein \\
\hline Caspr2 & Contactin-associated protein-2 \\
\hline $\mathrm{CF}$ & climbing fibers \\
\hline CGT & ceramide galactosyltransferase \\
\hline
\end{tabular}




\begin{tabular}{|c|c|}
\hline CMT & Charcot-Marie Tooth disease \\
\hline CNS & central nervous system \\
\hline Cont & Contactin \\
\hline Cora & Coracle \\
\hline CRMP-2 & collapsin response mediator protein- 2 \\
\hline CTD & c-terminal domain \\
\hline DRG & dorsal root ganglion \\
\hline EBP50 & ezrin binding protein- 50 \\
\hline $\mathrm{ECM}$ & extracellular matrix \\
\hline EDTA & Ethylene-diamine-tetra-acetic acid \\
\hline EGTA & Ethylene-glycol-tetra-acetic acid \\
\hline EM & electron microscopy \\
\hline EPSC & excitatory postsynaptic current \\
\hline ES cells & embryonic stem cells \\
\hline FERM & 4.1/ezrin/radixin/moesin \\
\hline GABA & $\gamma$-aminobutyric acid \\
\hline GAD65 & Glutamate decarboxylase- 65 \\
\hline GFP & green fluorescent protein \\
\hline GPI & Glycosylphosphatidylinositol \\
\hline HEPES & hydroxylethyl piperazeethanesulfonic acid \\
\hline HRP & horse-radish peroxidase \\
\hline Ig & immunoglobulin \\
\hline IKK & inhibitory $\kappa \mathrm{B}$ kinase \\
\hline
\end{tabular}




\begin{tabular}{|c|c|}
\hline IP & immunoprecipitation \\
\hline IPSC & inhibitory postsynaptic current \\
\hline JXP & juxtaparanode \\
\hline $\mathrm{K}_{\mathrm{V}}$ & potassium channels \\
\hline MAG & myelin-associated glycoprotein \\
\hline MAGUK & membrane-associated guanylate kinase \\
\hline MAPK & mitogen activated protein kinase \\
\hline MBP & myelin basic protein \\
\hline mIPSC & mini inhibitory postsynaptic current \\
\hline MS & multiple sclerosis \\
\hline $\mathrm{Na}_{\vee} 1 . \mathrm{x}$ & sodium channels \\
\hline NBQX & $\begin{array}{l}\text { 2,3-dihydroxy-6-nitro-7-sulfamoyl-benzo-quinoxaline-2,3-dione } \\
\text { AMPA receptor antagonist }\end{array}$ \\
\hline Necl-X & Nectin-like protein \\
\hline Nfasc/NFAS & Neurofascin \\
\hline $\mathrm{Nfasc}^{\mathrm{NF155}}$ & $155 \mathrm{kDa}$ isoform of Nfasc \\
\hline $\mathrm{Nfasc}^{\mathrm{NF} 186}$ & $186 \mathrm{kDa}$ isoform of Nfasc \\
\hline Nfasc ${ }^{\text {Flox }}$ & Nfasc floxed allele for conditional $N f a s c$ ablation \\
\hline $\mathrm{NF}-\kappa \mathrm{B}$ & nuclear factor kappa-light-chain-enhancer of activated B cells \\
\hline NMDA & N-Methyl-D-aspartate \\
\hline node & node of Ranvier \\
\hline $\mathrm{NrCAM}$ & neuron-related cell adhesion molecule \\
\hline NrxIV & Neurexin IV \\
\hline Nrg1 & Neuregulin 1 \\
\hline
\end{tabular}




\begin{tabular}{|c|c|}
\hline P\#\# & postnatal day \#\# \\
\hline Parv & Parvalbumin \\
\hline Pcp2 & Purkinje cell protein 2 \\
\hline PCR & polymerase chain reaction \\
\hline PBS & phosphate buffer saline \\
\hline PDZ & $\begin{array}{l}\text { Postsynaptic density protein 95/Drosophila disc large tumor } \\
\text { suppressor/Zonula occludens-1 protein }\end{array}$ \\
\hline PF & parallel fibers of the cerebellum \\
\hline $\mathrm{pI \kappa \textrm {B } \alpha}$ & phosphorylated inhibitor of $\kappa \mathrm{B} \alpha$ \\
\hline PLP & proteolipid protein \\
\hline pNfl & phosphorylated neurofilaments \\
\hline PNS & peripheral nervous system \\
\hline PSD-93 & postsynaptic density protein-93 \\
\hline PSD-95 & postsynaptic density protein-95 \\
\hline RNAi & RNA interference \\
\hline RT & room temperature \\
\hline RT-PCR & reverse transcription polymerase chain reaction \\
\hline SAB & spectrin/actin binding \\
\hline SDS & sodium dodecyl sulfate \\
\hline SEM & standard error of the mean \\
\hline shRNA & short hairpin RNA \\
\hline sIPSC & spontaneous inhibitory postsynaptic current \\
\hline Tag1 & transiently expressed axonal surface glycoprotein-1 \\
\hline TEM & transmission electron microscopy \\
\hline
\end{tabular}


TTX tetrodotoxin, Nav channel blocker

U1-U3 unique domains 1-3 of the family of protein 4.1

vGlut vesicular glutamate transporter

WT wild type

x $g$ times gravity 


\section{Chapter 1}

\section{Introduction: Organization and Maintenance of Molecular Domains in Myelinated Axons}

Over a century ago, Ramon y Cajal first proposed the idea that there is directionality involved in nerve conduction and neuronal communication (Ramon y Cajal, 1911). He further suggested that there needed to be a form of insulation around axons to allow for the fast conduction of action potentials. A few decades later, it was discovered that myelin, made from glial cells, insulated axons with periodic breaks where nodes of Ranvier (nodes) form to allow for saltatory conduction (Huxley and Stampfli, 1949; Tasaki, 1959). In the peripheral nervous system (PNS), Schwann cells are the glia that can either individually myelinate the axon from one neuron, or ensheath the axons of many neurons (Jessen and Mirsky, 2005). In the central nervous system (CNS), oligodendrocytes are the glia that myelinate regions of many different axons from many neurons at once (Simons and Trotter, 2007). Review of more recent studies revealed that this myelination created polarized domains adjacent to the nodes (Bhat, 2003; Salzer, 2003). However, the molecular players responsible for the organization of axonal domains are only now beginning to be elucidated.

The molecular domains in myelinated axons include the axon initial segment (AIS), where various ion channels are clustered and action potentials are initiated, the node, where sodium channels are clustered and action potentials are propagated, the 
paranode, where myelin loops make contact with the axolemma, the juxtaparanode (JXP), where delayed-rectifier potassium channels are clustered, and the internode, where myelin is most compactly wrapped (Fig. 1.1). Each of these domains contains a unique subset of proteins that are critical for its function and the proper segregation of these domains allows for normal neuronal communication (Table 1.1). However, the roles of these proteins in axonal domain organization are still being elucidated. Thus, I undertook studies to elucidate mechanisms responsible for the organization of axonal domains in myelinated axons.

At the paranode, myelin loops make contact with the paranodal axolemma through axo-glial septate junctions (AGSJs), which are also critical for the organization of the axonal cytoskeleton. However, it was not known how the AGSJs interact with the axonal cytoskeleton. I addressed this question by examining the cytoskeletal adaptor protein 4.1B in the role of the axonal cytoskeleton in AGSJ formation and stabilization. While paranode organization requires myelination, the AIS forms intrinsically, independent of myelin (Alessandri-Haber et al., 2002; Winckler et al., 1999; Zhang and Bennett, 1998). However, the mechanisms responsible for AIS organization are poorly understood. To address this question, I investigated the role of Neurofascin (Nfasc) in AIS formation and stabilization. Further, I sought to understand the role of Nfasc in the organization of the cerebella pinceau, a structure that is critical for regulating AIS function. The results from these studies are presented in this dissertation and reveal novel mechanisms involved in axonal domain organization. First, I highlight a few of the important interactions for axonal domain organization and their role in maintaining proper neuronal communication. 


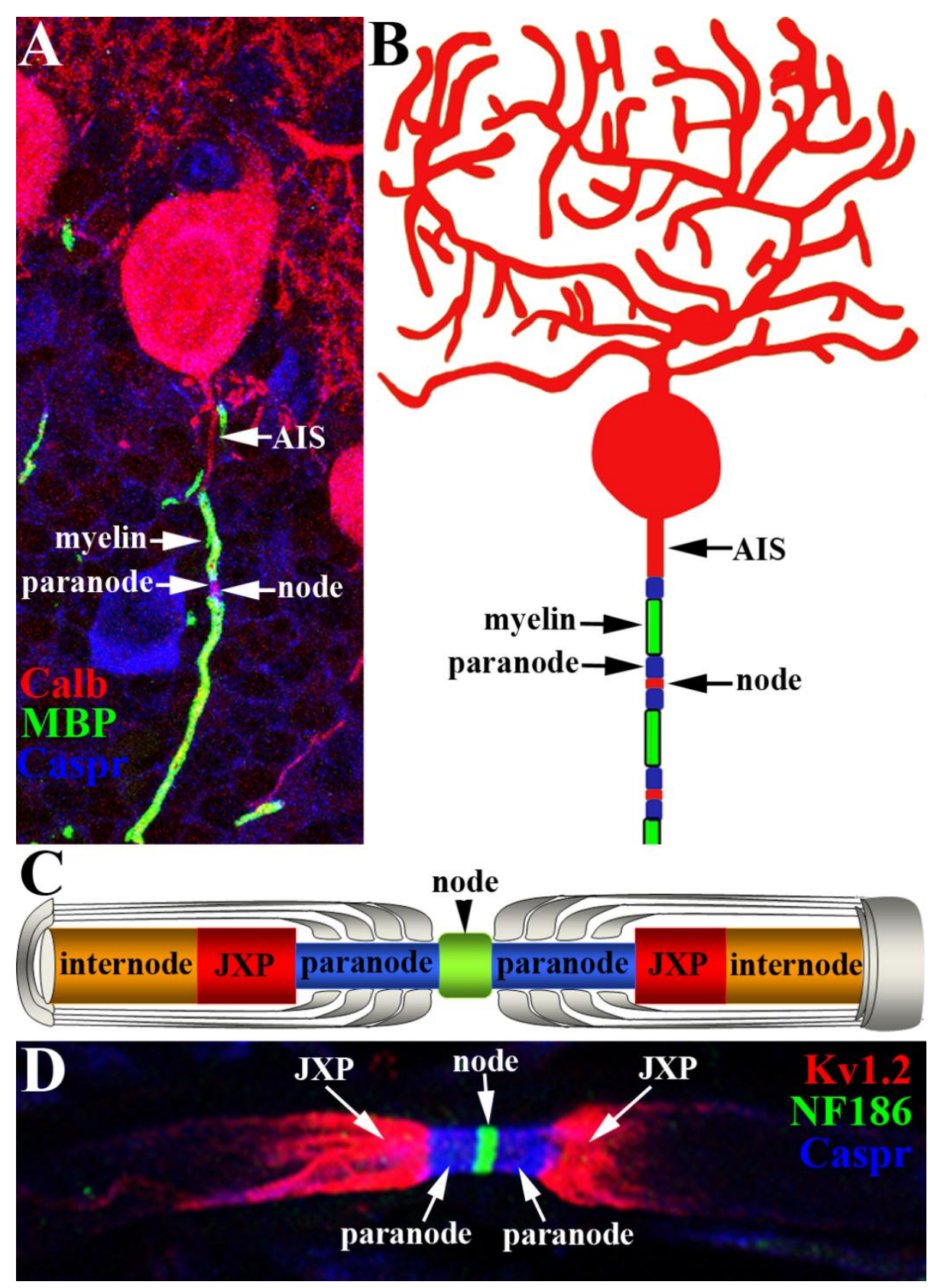

\section{Figure 1.1 Molecular domains in myelinated axons}

(A) Immunostaining of 1-month-old mouse cerebellar Purkinje neuron immunostained with Calbindin (Calb, red), myelin basic protein (MBP, green), and Contactin-associated protein (Caspr, blue). (B) Schematic of a Purkinje neuron highlighting the AIS, myelinwrapped internode, paranode, and node. (C) Schematic of nodal area highlighting the internode (orange), JXP (red), paranode (blue), and node (green). (D) Immunostaining of a 1-month-old mouse sciatic nerve fiber immunostained against potassium channels to label the JXP (red), Caspr to label the paranode (blue), and the 186kDa isoform of Nfasc to label the node (green). 


\begin{tabular}{|c|c|c|c|}
\hline Protein & Abbreviation & $\begin{array}{l}\text { Expressed } \\
\text { in }\end{array}$ & $\begin{array}{l}\text { Known functions in molecular } \\
\text { domains of myelinated axons }\end{array}$ \\
\hline $\begin{array}{l}\text { Neurofascin } \\
186 \mathrm{kDa}\end{array}$ & Nfasc $^{\text {NF186 }}$ & $\begin{array}{l}\text { Neuron at } \\
\text { AIS and } \\
\text { node }\end{array}$ & $\begin{array}{l}\text { Cell adhesion molecule important } \\
\text { for formation of the node and } \\
\text { maturation/stabilization of the AIS. }\end{array}$ \\
\hline AnkyrinG & AnkG & $\begin{array}{l}\text { Neuron at } \\
\text { AIS and } \\
\text { node }\end{array}$ & $\begin{array}{l}\text { Cytoskeletal adaptor protein that } \\
\text { links membrane proteins to } \\
\text { spectrin-actin cytoskeleton. } \\
\text { Important for formation of the AIS } \\
\text { and stabilization of the node. }\end{array}$ \\
\hline $\begin{array}{l}\text { Voltage-gated } \\
\text { sodium } \\
\text { channels }\end{array}$ & Nav1.x & $\begin{array}{l}\text { Neuron at } \\
\text { AIS and } \\
\text { node }\end{array}$ & $\begin{array}{l}\text { Sodium ion influx responsible for } \\
\text { action potential initiation and } \\
\text { propagation. }\end{array}$ \\
\hline $\begin{array}{l}\text { Voltage-gated } \\
\text { potassium } \\
\text { channels }\end{array}$ & $K_{V} 1 . x$ & $\begin{array}{l}\text { Neuron at } \\
\text { AIS and } \\
\text { JXP }\end{array}$ & $\begin{array}{l}\text { Potassium ion out flux to modulate } \\
\text { neuronal excitability and axonal } \\
\text { membrane potential. }\end{array}$ \\
\hline $\begin{array}{l}\text { Neuron-related } \\
\text { cell adhesion } \\
\text { molecule }\end{array}$ & NrCAM & $\begin{array}{l}\text { Neuron at } \\
\text { AIS and } \\
\text { node }\end{array}$ & $\begin{array}{l}\text { Cell adhesion molecule important } \\
\text { for neurite outgrowth and } \\
\text { intercellular adhesion. }\end{array}$ \\
\hline $\begin{array}{l}\text { Contactin- } \\
\text { associated } \\
\text { protein } 2\end{array}$ & Caspr2 & $\begin{array}{l}\text { Neuron at } \\
\text { AIS and } \\
\text { JXP }\end{array}$ & $\begin{array}{l}\text { Cell adhesion molecular important } \\
\text { for the organization of the JXP and } \\
\text { intercellular adhesion. }\end{array}$ \\
\hline $\begin{array}{l}\text { Transiently } \\
\text { expressed } \\
\text { axonal surface } \\
\text { glycoprotein-1 }\end{array}$ & Tag-1 & $\begin{array}{l}\text { Neuron at } \\
\text { AIS and } \\
\text { JXP and } \\
\text { glial cell at } \\
\text { JXP }\end{array}$ & $\begin{array}{l}\text { GPI-anchored cell adhesion } \\
\text { molecule important for organization } \\
\text { of the JXP and intercellular } \\
\text { adhesion. }\end{array}$ \\
\hline$\beta I V$-spectrin & $\beta I V$-spectrin & $\begin{array}{l}\text { Neuron at } \\
\text { AIS and } \\
\text { node }\end{array}$ & $\begin{array}{l}\text { Cytoskeletal scaffolding protein } \\
\text { that links actin to membrane-bound } \\
\text { proteins at the AIS and node. }\end{array}$ \\
\hline $\begin{array}{l}\text { Postsynaptic } \\
\text { density protein - } \\
93 \text { and }-95\end{array}$ & $\begin{array}{l}\text { PSD93/ } \\
\text { PSD95 }\end{array}$ & $\begin{array}{l}\text { Neuron at } \\
\text { AIS and } \\
\text { JXP }\end{array}$ & $\begin{array}{l}\text { MAGUK proteins that scaffold ion } \\
\text { channels and receptors at the AIS } \\
\text { and JXP. }\end{array}$ \\
\hline Gliomedin & GLDN & $\begin{array}{l}\text { Schwann } \\
\text { cell at node }\end{array}$ & $\begin{array}{l}\text { Helps cluster Nfasc }{ }^{\mathrm{NF} 186} \text { at the PNS } \\
\text { node. }\end{array}$ \\
\hline $\begin{array}{l}\text { Ezrin binding } \\
\text { protein } 50\end{array}$ & EBP50 & $\begin{array}{l}\text { Schwann } \\
\text { cell at node }\end{array}$ & $\begin{array}{l}\text { ERM-family protein that localizes } \\
\text { to the node and binds actin } \\
\text { cytoskeleton. }\end{array}$ \\
\hline $\begin{array}{l}\text { Contactin- } \\
\text { associated } \\
\text { protein }\end{array}$ & Caspr & $\begin{array}{l}\text { Neuron at } \\
\text { paranode }\end{array}$ & $\begin{array}{l}\text { Cell adhesion molecule that is one } \\
\text { of the required components of the } \\
\text { AGSJs. }\end{array}$ \\
\hline Contactin & Cont & $\begin{array}{l}\text { Neuron at } \\
\text { paranode }\end{array}$ & $\begin{array}{l}\text { GPI-anchored cell adhesion } \\
\text { molecule that is one of the required } \\
\text { components of the AGSJs. }\end{array}$ \\
\hline $\begin{array}{l}\text { Neurofascin } \\
155 \mathrm{kDa}\end{array}$ & Nfasc $^{\text {NF155 }}$ & $\begin{array}{l}\text { Glial cell at } \\
\text { paranode }\end{array}$ & $\begin{array}{l}\text { Cell adhesion molecule localized to } \\
\text { glial membrane and is a component }\end{array}$ \\
\hline
\end{tabular}




\begin{tabular}{|c|c|c|c|}
\hline & & & of the paranodal AGSJs. \\
\hline $\begin{array}{l}\alpha \mathrm{II}-\text { and } \beta \mathrm{II}- \\
\text { spectrin }\end{array}$ & $\begin{array}{l}\alpha I I / \beta I I- \\
\text { spectrin }\end{array}$ & $\begin{array}{l}\text { Neuron at } \\
\text { paranode }\end{array}$ & $\begin{array}{l}\text { Cytoskeletal binding proteins that } \\
\text { function to link the actin } \\
\text { cytoskeleton to the membrane- } \\
\text { bound AGSJs. }\end{array}$ \\
\hline Protein 4.1B & 4.1B & $\begin{array}{l}\text { Neuron at } \\
\text { paranode } \\
\text { and JXP }\end{array}$ & $\begin{array}{l}\text { Cytoskeletal adaptor protein } \\
\text { important for organization of JXP } \\
\text { and stabilization of paranodal } \\
\text { AGSJs. }\end{array}$ \\
\hline AnkyrinB & AnkB & $\begin{array}{l}\text { Neuron at } \\
\text { paranode }\end{array}$ & $\begin{array}{l}\text { Cytoskeletal adaptor protein that } \\
\text { links membrane proteins to the } \\
\text { spectrin-actin cytoskeleton at the } \\
\text { paranode. }\end{array}$ \\
\hline $\begin{array}{l}\text { Ceramide } \\
\text { galactosyltrans- } \\
\text { ferase }\end{array}$ & CGT & $\begin{array}{l}\text { Glial cell at } \\
\text { paranode }\end{array}$ & $\begin{array}{l}\text { Enzyme important for lipid } \\
\text { biosynthesis that is important for } \\
\text { paranode stabilization. }\end{array}$ \\
\hline $\begin{array}{l}\text { Myelin } \\
\text { associated } \\
\text { glycoprotein }\end{array}$ & MAG & $\begin{array}{l}\text { Oligo- } \\
\text { dendrocyte } \\
\text { at the } \\
\text { internode }\end{array}$ & $\begin{array}{l}\text { Glycoprotein localized to the cell } \\
\text { membrane that is important for } \\
\text { myelination. }\end{array}$ \\
\hline $\begin{array}{l}\text { Nectin-like-x } \\
\text { protein }\end{array}$ & Necl-x & $\begin{array}{l}\text { Neuron and } \\
\text { glia at } \\
\text { internode }\end{array}$ & $\begin{array}{l}\text { Cell adhesion molecule important } \\
\text { for intermodal adhesion. }\end{array}$ \\
\hline Protein 4.1G & $4.1 \mathrm{G}$ & $\begin{array}{l}\text { Schwann } \\
\text { cells at the } \\
\text { internode }\end{array}$ & $\begin{array}{l}\text { Cytoskeletal adaptor protein } \\
\text { important for internode and JXP } \\
\text { organization. }\end{array}$ \\
\hline $\begin{array}{l}\text { Proteolipid } \\
\text { protein }\end{array}$ & PLP & $\begin{array}{l}\text { Oligo- } \\
\text { dendrocyte } \\
\text { at internode }\end{array}$ & $\begin{array}{l}\text { Transmembrane protein that forms } \\
\text { homomeric complex with itself } \\
\text { across myelin loops to stabilize } \\
\text { compact myelin. }\end{array}$ \\
\hline $\begin{array}{l}\text { Myelin basic } \\
\text { protein }\end{array}$ & MBP & $\begin{array}{l}\text { Glial cell at } \\
\text { internode }\end{array}$ & $\begin{array}{l}\text { Important for myelination and } \\
\text { maintaining paranodal and nodal } \\
\text { organization. }\end{array}$ \\
\hline $\begin{array}{l}\text { Myelin protein } \\
\text { zero }\end{array}$ & P0 & $\begin{array}{l}\text { Schwann } \\
\text { cell at } \\
\text { internode }\end{array}$ & $\begin{array}{l}\text { Glycoprotein that is critical for PNS } \\
\text { myelin structure. }\end{array}$ \\
\hline
\end{tabular}

Table 1.1 Molecular components of molecular domains in myelinated axons.

This table is an incomplete listing of the molecular components of molecular domains in myelinated axons, including their function for domain organization. It highlights the best understood components of the AIS, node, paranode, JXP, and internode. 


\subsection{The axon initial segment (AIS)}

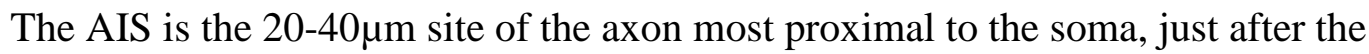
axon hillock (Fig. 1.1) (Hedstrom and Rasband, 2006). All of the excitatory and inhibitory inputs to the neuron are summated at the AIS and a decision is made whether or not to fire an action potential. Clustered to the AIS are ion channels, including sodium $\left(\mathrm{Na}^{+}\right)$channels responsible for action potential initiation, $\mathrm{Na}_{\mathrm{V}} 1.6$, and potassium channels, $\mathrm{K}_{\mathrm{V}} 1.1 / 1.2$ and $\mathrm{KCNQ} 2 / 3$, which are important for modulating neuronal excitability and regulating action potential frequency (Table 1.1; Fig. 1.2) (Johnston et al., 2008; Kole et al., 2008; Pan et al., 2006). There are also several cell adhesion molecules (CAMs), belonging to the L1 subfamily of immunoglobulin (Ig) CAMs, including the $186 \mathrm{kDa}$ isoform of Nfasc $\left(\mathrm{Nfasc}^{\mathrm{NF} 186}\right)$, neuron-related cell adhesion molecule (NrCAM), Contactin-associated protein-2 (Caspr2), and transiently expressed axonal surface glycoprotein-1 (Tag1) (Table 1.1; Fig. 1.2) (Brummendorf et al., 1998; Davis et al., 1996; Dodd et al., 1988; Grumet, 1997; Hedstrom et al., 2007; Volkmer et al., 1992). Intracellularly at the AIS, there are adaptor and scaffolding proteins, including AnkyrinG (AnkG), $\beta I V$-spectrin, and postsynaptic density protein 93 (PSD-93) (Table 1.1; Fig. 1.2) (Jenkins and Bennett, 2001; Kole et al., 2008). The adaptor protein AnkG binds membrane bound proteins, including voltage-gated sodium channels and CAMs and links them to the underlying actin/spectrin cytoskeleton through scaffolding proteins, including $\beta$ IV-spectrin (Fig. 1.2) (Boiko et al., 2007; Zhou et al., 1998). Nfasc ${ }^{\mathrm{NF} 186}$ and NrCAM are able to link to AnkG through a conserved motif that is mediated by tyrosine phosphorylation, such that the unphosphorylated motif can bind with AnkG (Davis et al., 1996; Garver et al., 1997; Lustig et al., 2001; Zhang et al., 1998). The accumulation of 
these complexes is necessary for clustering and stabilization of $\mathrm{Na}_{\mathrm{V}}$ channels at the AIS (Buttermore et al., 2012; Hedstrom et al., 2007; Zhou et al., 1998; Zonta et al., 2011). Interestingly, recent reports show that the AIS itself is segregated into proximal and distal domains based on the localization of different sodium channel isoforms with different voltage-sensitivities, such that $\mathrm{Na}_{\mathrm{V}} 1.2$ clusters at the proximal AIS and $\mathrm{Na}_{\mathrm{V}} 1.6$ is enriched at the distal AIS (Fig. 1.2) (Buttermore et al., 2012; Hu et al., 2009). Electrophysiological studies from the same group show that sodium channels localized to the distal AIS are activated at a lower voltage threshold than sodium channels at the proximal AIS (Hu et al., 2009). The authors suggest that the distal sodium channels, which have a higher-voltage threshold, are responsible for action potential initiation and the proximal sodium channels, which have a lower-voltage threshold, are responsible for action potential back-propagation. Thus, the segregation of these sodium channel isoforms and the precise organization of the AIS are critical for regulating axonal activity. Therefore, it is important to understand how the AIS is formed and maintained. 

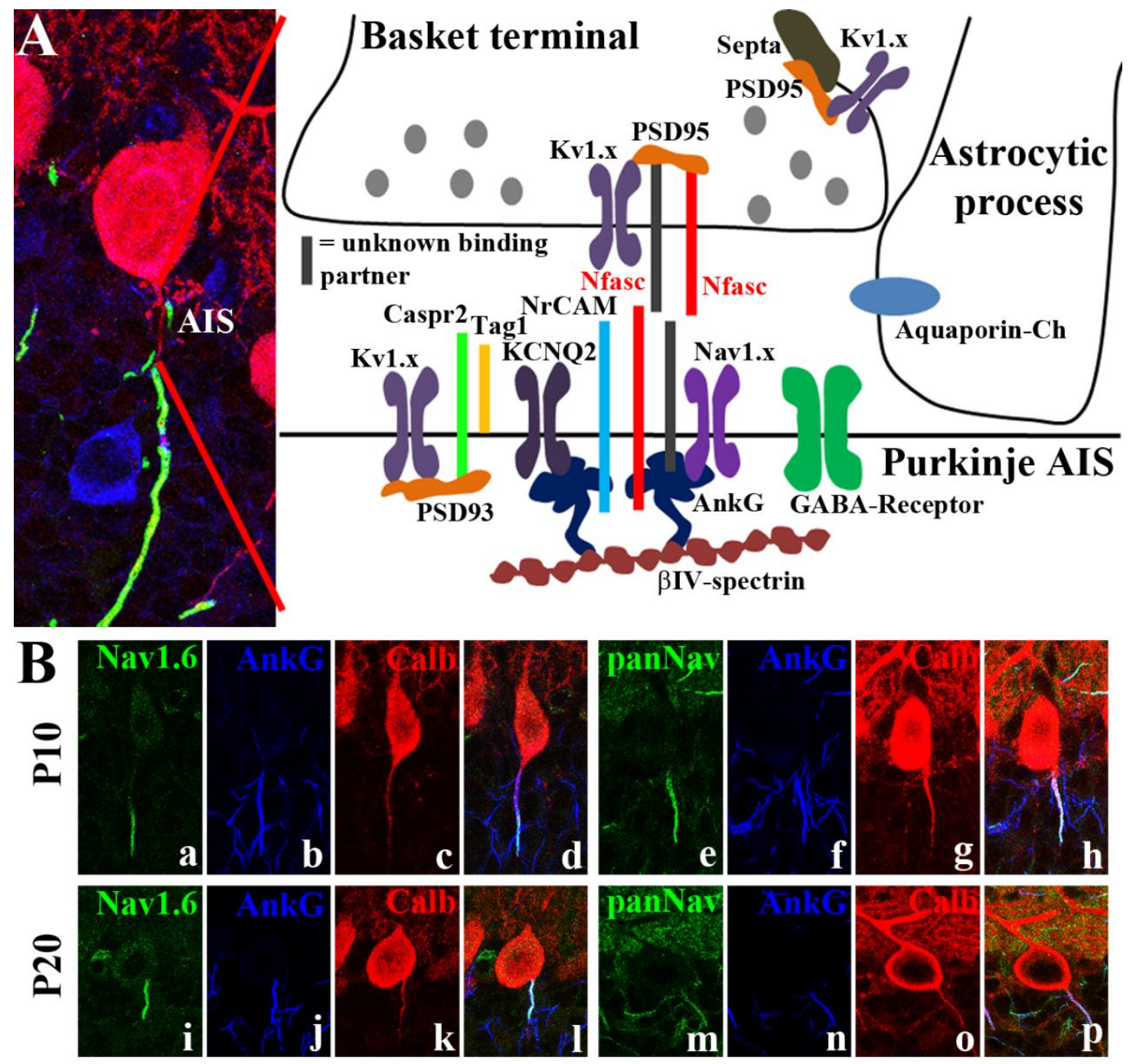

Figure 1.2 Molecular organization and maturation of the AIS

(A) Molecular components of the AIS, including the ion channels, CAMs and cytoskeletal scaffolding proteins that function at the AIS. The diagram also highlights some of the known components of the cerebellar pinceau (discussed below).

(B) Cerebellar sections of P10 and P20 wild type mice immunostained against Nav1.6 (a, i), panNav (e, m), AnkG (b, f, j, n), Calb (c, g, k, o) and merged (d, h, l, p). These immunostainings reveal the developmental maturation of the AIS, as Nav1.6 is distally localized to the AIS at P10 (a) and is fully localized to the AIS at P20 (i). 


\subsubsection{Formation of the AIS}

While the organization of most axonal domains requires intercellular communication, the organization of the AIS occurs intrinsically through the localization of the "AIS master regulator," AnkG (Bennett and Baines, 2001). Ankyrins are known for their ability to stabilize groups of membrane-bound proteins to specific molecular domains for efficient signaling and interactions between cells (Bennett and Baines, 2001). The critical role for AnkG in AIS organization was discovered in several independent in vitro and in vivo studies (Hedstrom et al., 2007; Zhou et al., 1998). In the first, knockdown of AnkG using shRNA in cultured hippocampal neurons resulted in failure of all other AIS components to cluster at the AIS (Hedstrom et al., 2007). Importantly, knockdown of other AIS components, including Nfasc, NrCAM and $\beta I V-$ spectrin, did not disrupt the enrichment of the other AIS components. Further, genetic ablation of $A n k G$ in the cerebellum resulted in loss of clustering of Nfasc and ion channels at the Purkinje AIS (Zhou et al., 1998). Together, these results point to a role for AnkG as the master regulator of the AIS. Importantly, loss of AnkG also results in disrupted axonal polarity, with the formation of spines and localization of dendritic proteins in Purkinje neuron AISs lacking AnkG (Sobotzik et al., 2009). One study showed that AnkG is also required for AIS stability by knocking down AnkG in mature cultured hippocampal neurons that already formed an AIS prior to shRNA treatment (Hedstrom et al., 2008). In these adult AnkG knockdown neurons, AIS markers were no longer clustered at the AIS and the process that had been the axon contained both axonal and dendritic markers, while the other processes only contained dendritic markers. Further, in vivo ablation of $A n k G$ from Purkinje neurons did not disrupt the ability of the 
Purkinje neuron to form an axon, but the axonal projection did contain dendritic spines (Sobotzik et al., 2009). Therefore, without AnkG present, the AIS does not form properly and axonal identity is lost. The signals responsible for AnkG clustering at the AIS are the focus of ongoing studies. One hypothesis suggests that phosphorylated inhibitor of kappa B alpha $(\kappa \mathrm{B} \alpha)$ may function as a cofactor in AnkG trafficking to the AIS (Rasband, 2010; Sanchez-Ponce et al., 2008; Schultz et al., 2006), but further studies are required to confirm these results in vivo.

\subsubsection{The AIS is a barrier for axonal transport}

The loss of axonal polarity and invasion of dendritic markers found in Purkinje neurons that lack AnkG reflects a role of the AIS as a sieve, preventing the diffusion of dendritic or somatic proteins into the axon. It also reflects the idea that dendritic fate of neuronal projections is the default choice and AnkG and the AIS are needed to maintain the barrier that maintains axonal identity. The role of the AIS as a diffusion barrier was first discovered when it was found that some membrane proteins diffused through the AIS slower than others (Winckler et al., 1999). Further, disruption of the F-actin cytoskeleton prevented this decreased mobility, suggesting that the AIS barrier depends on the interaction of membrane proteins with the cytoskeleton. Another more recent study followed individual fluorescently-tagged unsaturated phospholipids and found their diffusion is blocked in the AIS membrane at the same time in development that the clustering of AIS proteins occurs (Nakada et al., 2003). Importantly, the barrier function of the AIS restricts both membrane-bound and cytoplasmic proteins from diffusing into the axon. A more recent study found that larger dextrans could not diffuse into the axon 
after AIS formation, while smaller dextrans could diffuse in (Song et al., 2009). As in the previous studies, disruption of the F-actin cytoskeleton disrupted the function of the AIS barrier for cytoplasmic diffusion. Importantly, these authors also found that axonal entry of kinesin superfamily motor proteins was dependent on the cargo they carry, as dendritic cargos were prevented from entering the axon after the AIS was formed (Song et al., 2009). These results show that the AIS is not simply a structure for action potential initiation, it is also critical for maintaining axonal identity.

\subsubsection{Functional maturation of the AIS}

Though the initial organization of the AIS requires AnkG localization to this domain, our data shows that the maturation of the AIS depends on $\mathrm{Nfasc}^{\mathrm{NF} 186}$ expression (Buttermore et al., 2012). The developmental switch of $\mathrm{Na}_{\mathrm{V}} 1.2$ to $\mathrm{Na}_{\mathrm{V}} 1.6$ at the AIS is critical for AIS function in the adult myelinated axons (Boiko et al., 2003). In our recent work, we show that Purkinje neuron-specific ablation of $\mathrm{Nfasc}^{\mathrm{NF} 186}$ prevents the maturation of the Purkinje AIS (Buttermore et al., 2012). In the absence of Nfasc ${ }^{\mathrm{NF} 186}$, the mature voltage-gated sodium channel isoform, $\mathrm{Na}_{\vee} 1.6$, fails to become enriched at the Purkinje AIS, as it does at the wild-type Purkinje AIS. This suggests that different mechanisms are responsible for the initial formation and secondary maturation of the AIS. Further, recent studies completed by our lab and others have shown that an intact Purkinje AIS is not required for induction of Purkinje neuron firing (Buttermore et al., 2012; Zonta et al., 2011). However, the waveform of the resulting action potential is altered (Zonta et al., 2011). In addition, spontaneous firing of the Purkinje cell is disrupted with loss of the AIS (Buttermore et al., 2012; Zonta et al., 2011). These results 
show that functional maturation of the AIS is required for normal neuronal firing.

Fascinating recent studies have also shown that the AIS is a plastic structure that changes in response to neuronal activity (Grubb and Burrone, 2010a; Kuba et al., 2010).

Therefore, it is possible that the mechanisms responsible for changes in AIS structure during plastic changes are similar to those responsible for the maturation of the AIS.

\subsubsection{Plasticity of the AIS}

The discovery that the AIS is a plastic structure reiterates the importance of the AIS in the regulation of neuronal activity. Previous studies eluded to the idea that the AIS is not a static structure, as the tuning frequency, AIS size and location of the AIS differ between neuronal subtypes and brain regions, depending on the requirement of the individual neuron within its circuit (Fried et al., 2009; Grubb et al., 2011; Konishi, 2003; Kuba et al., 2006; Lorincz and Nusser, 2008). However, the first direct evidence of AIS plasticity was not revealed until more recently in two separate studies. In one study, auditory deprivation led to an increase in AIS length in auditory neurons of the avian brainstem (Kuba et al., 2010). In the other study, evidence for AIS plasticity was uncovered through recent technological advances that allow for live image of a functioning AIS through imaging of ion flux (Grubb and Burrone, 2010a; Grubb et al., 2011). This study revealed that the AIS can shift its position distally along the axon in vitro when it is exposed to increased excitatory activity over a long period (Grubb and Burrone, 2010a). This shift in position of the AIS occurred after two days of prolonged stimulation and required voltage-gated calcium channel activity. These results suggest that the AIS is able to react to its environment so that it can control its firing rate, in this 
case decreasing the probability of firing by moving further down this axon. Thus, the AIS is able to help the axon regulate neuronal activity. However, it remains to be seen whether this AIS plasticity is found in older adult stages as well. It may be that the AIS becomes more stable in adult stages. $\mathrm{Nfasc}^{\mathrm{NF} 186}$ has also been shown to be critical for the long-term stabilization of the AIS (Zonta et al., 2011). It is likely that mechanisms are also in place to maintain the proper organization of the AIS, as its proper functioning is critical for normal neuronal activity.

\subsubsection{Regulation of AIS function}

While cell-autonomous mechanisms may be responsible for the organization and plasticity of the AIS, the AIS is frequently targeted by inhibitory interneurons to aid in modulating neuronal firing. Examples of this can be found in the targeting of chandelier cells to the pyramidal AIS in the cortex and basket cell targeting to the Purkinje AIS in the cerebellum. These interactions have been shown to be critical for proper function of the targeted neuron. For example, the function of the cerebellum is to regulate motor coordination and the Purkinje cell summates all inputs to the cerebellum and is the sole output source of the cerebellum, so its activity must be tightly regulated (Palay and Palay, 1974). This restriction is achieved, in part, by the basket cell, which is an inhibitory interneuron in the molecular layer that sends projections to the Purkinje AIS (Fig. 1.3). An extraordinary feature of the basket cell is its axon, which splits into many axonal collaterals that innervate 5 to 7 Purkinje cells. Basket axon collaterals extend around the Purkinje soma, synapsing on the Purkinje AIS and forming the pinceau (Fig. 1.3). Inhibition of the Purkinje AIS is achieved through $\gamma$-aminobutyric acid- (GABA)-ergic 
synapses that basket axons form with the Purkinje neuron, releasing the inhibitory neurotransmitter GABA to dampen Purkinje cell excitability (Purves et al., 2004). A single basket cell innervates several Purkinje cells, allowing for coordinated modulation of a group of Purkinje cells (Ango et al., 2004; Cobb et al., 1995). Potassium channels $\left(\mathrm{K}_{\mathrm{V}} 1.2\right)$ are also enriched at the basket axon terminals of the pinceau (Fig. 1.3) (Laube et al., 1996). Importantly, the pinceau has been shown to play a critical role in Purkinje neuron function. One study found that disruption of pinceau organization results in ataxia, a consequence of Purkinje neuron dysfunction (Xie et al., 2010). In this study, the authors discovered a mouse with a mutation in $\mathrm{K}_{\mathrm{V}} 1.2$ channels and found this disrupted the pinceau structure and cerebella activity, resulting in ataxia. Interestingly, loss of $\mathrm{K}_{\mathrm{V}} 1.2$ function in basket interneurons in the cerebellum resulted in hyperactive basket neurons, which resulted in decreased Purkinje neuron firing. So it seems that $\mathrm{K}_{\mathrm{V}} 1.2$ channels in basket neurons are functioning to dampen neuronal excitability at the pinceau. Upon re-expression of the potassium channels, the ataxia phenotype was partially rescued, reiterating the importance of the inhibitory balance at the pinceau on Purkinje neuron function.

While the Purkinje neuron depends on the pinceau for proper function, the Purkinje AIS also plays an important role in the developmental formation and long-term maintenance of the pinceau (Ango et al., 2004; Buttermore et al., 2012; Zonta et al., 2011). In one study, the authors found that ablation of $A n k G$ resulted in mislocalization of Nfasc distally down the Purkinje axon, with basket axons following aberrantly localized Nfasc (Ango et al., 2004). In addition, expression of a dominant-negative Nfasc construct in Purkinje neurons prevented the clustering of synaptic marker glutamate decarboxylase- 
65 (GAD65) at the pinceau (Ango et al., 2004). More recently, it was shown that ablation of $N$ fasc in adult neurons results in destabilization of the pinceau after 16 weeks (Zonta et al., 2011). Finally, our recent studies utilized cell-specific ablation of Nfasc to show that Nfasc is required in both Purkinje neurons and basket neurons for pinceau organization (Buttermore et al., 2012). We found that Nfasc in the Purkinje AIS is required for the stabilization and maintenance of the interaction between the basket axon terminals and the Purkinje axon. We also showed that in the basket axon, Nfasc is likely required for proper basket axon branching and outgrowth towards the Purkinje AIS. Interestingly, a role for Nfasc in GABAergic synapse organization was also found in the developing hippocampus, where Nfasc induces clustering of gephyrin, a postsynaptic scaffolding protein required for recruiting GABA receptors, at the future AIS (Burkarth et al., 2007). In addition, in the adult dentate gyrus, Nfasc stabilizes GABAergic synaptic components (Kriebel et al., 2011). Further, Nfasc has also been shown to cluster the extracellular matrix (ECM) molecule Brevican to the extracellular space surrounding the AIS, suggesting the AIS plays a role in the organization of the ECM (Hedstrom et al., 2007). The ECM surrounding the AIS may be important for buffering ions in the area, or for maintaining synaptic contact, as perineuronal nets have been shown to function elsewhere in the brain (Celio et al., 1998; Hedstrom et al., 2007). Together, these data reveal a conserved mechanism in which AIS organization is critical for targeting of GABAergic synapses for proper regulation of the postsynaptic neuron. Therefore, although the AIS is the one axonal domain that forms independent of other cellular influence, its proper function relies heavily on synaptic input and modulation from its extracellular environment. 

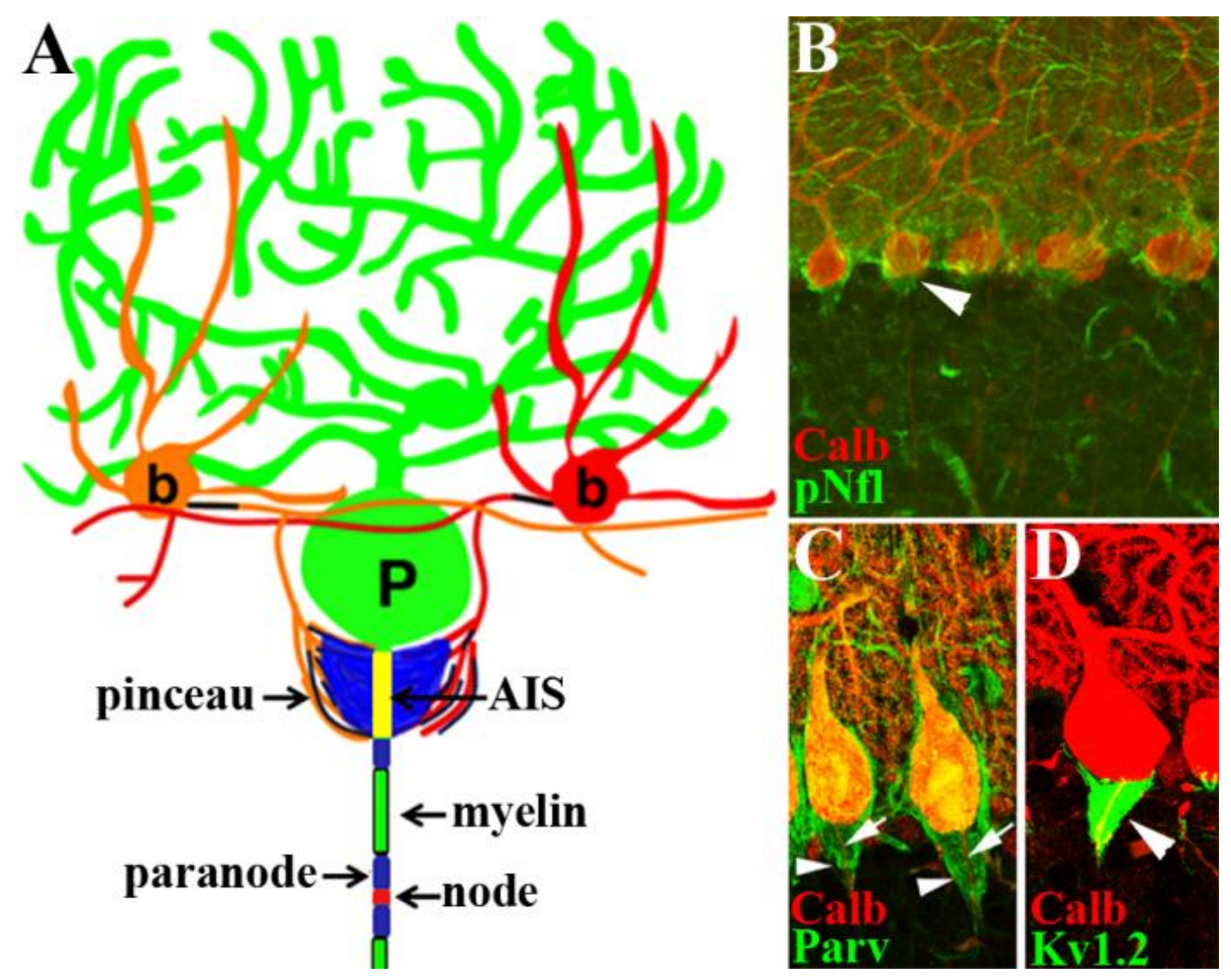

\section{Figure 1.3 Organization of the cerebellar pinceau}

(A) Drawing of the cerebellar pinceau highlighting the Purkinje neuron (P) in green, basket neurons (b) in red and orange, potassium channels in blue, and the AIS in yellow. (B) Low magnification image of a cerebellar section immunostained against Calb (red) and phosphorylates neurofilaments (pNfls, green). The pNfls label the basket axons and the pinceau (arrowhead).

(C) High magnification image of cerebellar section immunostained against Calb (red) and Parv (green). Parv is localized to both Purkinje and basket neurons and highlights the basket collaterals that form the cone-shaped pinceau (arrowhead) at the Purkinje AIS (arrow).

(D) Cerebellar section immunostained against Calb (red) and $\mathrm{K}_{\mathrm{V}} 1.2$ (green) to label the inner core of the pinceau (arrowhead) at the Purkinje AIS. 


\subsubsection{AIS in disease and injury}

The importance of in vivo genetic models in supplement to in vitro culture systems for elucidating the mechanisms by which the AIS is organized and maintained are underlined by a recent report that found the AIS is a target for injury during ischemia (Schafer et al., 2009). This study found that during stroke, the protease calpain proteolyzed AnkG and $\beta I V$-spectrin, which disrupted the AIS, resulting in loss of neuronal polarity. This damage to the AIS resulted independently of axonal degeneration or programmed cell death, showing that the AIS is a targeted site for axonal damage. Dysfunction of the AIS has also been found in diseased brain states. In one study, the AISs of pyramidal neurons in the hippocampus of Angelman Syndrome mice were found to be elongated and intrinsic membrane properties were altered (Kaphzan et al., 2011). The initial resting potential of neurons in the Angelman Syndrome mice was more hyperpolarized, due to increased sodium/potassium channel pumps. In addition, action potential amplitude and its maximal rate of rise were larger in Angelman Syndrome mice, due to the increased $\mathrm{Na}_{\mathrm{V}} 1.6$ and AnkG found at the AIS in the hippocampus, but not somatosensory cortex. Together, these alterations resulted in decreased neuronal excitability (Kaphzan et al., 2011). Therefore, it is critical to maintain the balance of ion channel expression at the AIS for proper neuronal function. Further, we need to understand how the AIS is formed and maintained to begin thinking about therapies for AIS dysfunction and injury.

In schizophrenic patients, researchers have found a decrease in GABA release at the synapses between chandelier neurons and the cortical pyramidal AIS (Lewis et al., 2005; Rasband, 2010). While the mechanisms responsible for this decrease in GABA 
transmission have not been elucidated, another study found that there is a decrease in AnkG in the superficial cortical layers of schizophrenic patients (Cruz et al., 2009). And as previously stated, a decrease in AnkG disrupts localization of AIS components, including Nfasc, and disruption of Nfasc alters GABA receptor clustering and targeting of GABAergic synapses to the AIS (Ango et al., 2004; Burkarth et al., 2007; Buttermore et al., 2012; Cruz et al., 2009). Therefore, it is possible that alterations in AIS stability and function may play a role in the imbalance of neuronal activity that is found in schizophrenic patients.

While imbalances in excitatory and inhibitory transmission throughout the brain are thought to be at the root of psychiatric disorders such as autism and schizophrenia, this imbalance can also cause other problems, such as epilepsy and seizures. It is well known that sodium channel mutations can cause epilepsy. One study found that the reason for the seizure activity in animals with $\beta_{1}$-subunit $\mathrm{Na}_{\mathrm{V}}$ channel mutations was that the mutation resulted in altered subcellular localization of the channels, resulting in hyper-excitable neurons (Meadows et al., 2002). Importantly, another study found that AnkG and Nav1.6 levels were increased at the AIS in an animal model for epilepsy (Chen et al., 2009). Therefore, a conserved mechanism for seizure activity seems to include an increase in sodium channel activity, possibly at the AIS. Each of these examples reiterates the fact that a balance of ion channel function is required within the axon, including the AIS, for proper neuronal function. Therefore, we must continue to elucidate the mechanisms responsible for ion channel localization and stabilization to the AIS so that therapies can be generated. 


\subsection{The node of Ranvier (node)}

Like the AIS, nodes are domains without myelin that are enriched with voltagegated sodium channels, critical for action potential propagation, though they are only about $1 \mu \mathrm{m}$ long (Fig. 1.1) (Hedstrom and Rasband, 2006; Rasband, 2006; Salzer, 2003; Thaxton and Bhat, 2009; Waxman and Ritchie, 1993). Action potentials initiated in the AIS jump from node to node by way of sodium channel currents from the channels clustered to these domains. Therefore, the segregation of sodium channels to the node is a critical process during the development of myelinated axons. Interestingly, many of the proteins that localize to the AIS are also localized to the node, including Nav1.2, Nav1.6, KCNQ2/3, $\beta I V$-spectrin, AnkG, NrCAM, and Nfasc ${ }^{\mathrm{NF} 186}$ (Table 1.1) (Berghs et al., 2000; Boiko et al., 2001; Komada and Soriano, 2002; Salzer, 2003; Volkmer et al., 1992). Similar to the AIS, AnkG interacts with $\mathrm{Na}_{\mathrm{v}} 1.6, \mathrm{CAMs}$ and the underlying cytoskeleton through $\beta I V$-spectrin at the node (Fig. 1.4) (Bennett and Lambert, 1999). Also similar to the AIS, there is a developmental switch in $\mathrm{Na}_{\mathrm{V}}$ channel isoform expression, from $\mathrm{Na}_{\mathrm{V}} 1.2$ in immature nodes to Nav1.6 in mature nodes (Boiko et al., 2001; Kaplan et al., 2001). However, the mechanism of node organization is quite different than that of the AIS.

The spacing of nodes, as well as their assembly, is largely coordinated with the help of myelinating glia (Court et al., 2004; Susuki and Rasband, 2008; Thaxton et al., 2011). In some regions of the nervous system, the spacing of nodes is critical for their function. For example, in the avian brainstem the spacing between nodes and the diameter of axons are controlled so that interaural time differences can be detected (Jeffress, 1948; Rasband, 2010; Seidl et al., 2010). In addition, the spacing of nodes is coordinated to allow for decreased membrane capacitance and increased membrane 
resistance, the necessary ingredients for saltatory conduction (Hille, 2001). For these reasons, many studies have focused on understanding how nodes are organized.

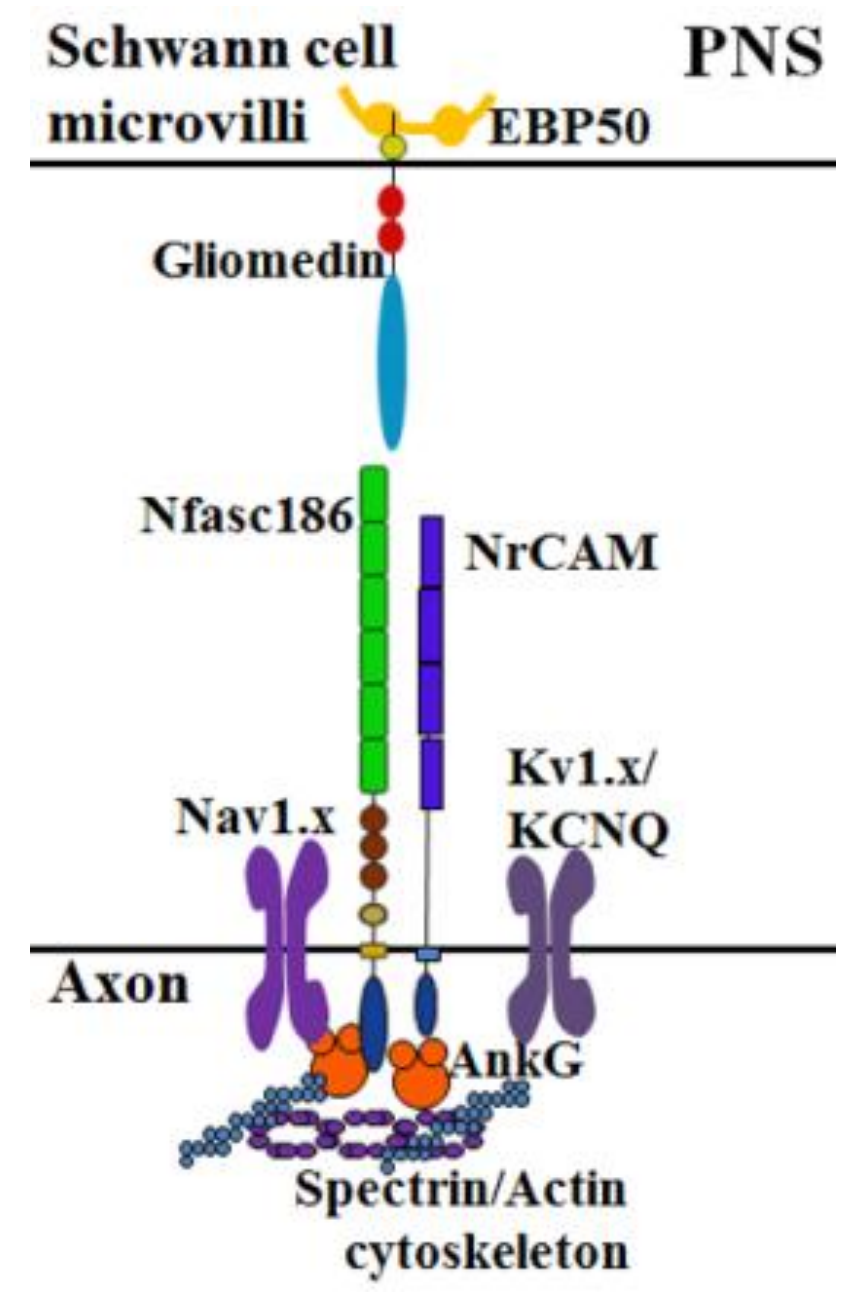

Figure 1.4 Molecular components of the PNS node

Diagram highlights the major molecular components of the PNS node. In the CNS, oligodendrocytes do not have microvilli and the binding partner for Nfasc is unknown. 


\subsubsection{Formation of the node}

While the AIS forms intrinsically, depending on the localization of AnkG before sodium channels and CAMs become clustered, the node develops with the aid of external signals (Eshed et al., 2005; Pedraza et al., 2001). Interestingly, the mechanisms of node formation in the PNS versus CNS are thought to occur differently, likely due to the differences in glial contribution to the process. In the PNS, Schwann cells produce proteins that localize to Schwann cell microvilli, protrusions that extend from the Schwann cell towards the nodal gap, and proteins that are secreted to become part of the ECM (Fig. 1.4) (Eshed et al., 2007; Eshed et al., 2005). The nodal ECM contains various glycoproteins that are important for stabilizing Schwann cell microvilli at the node (Melendez-Vasquez et al., 2005; Saito et al., 2003). It is generally agreed upon that initial binding of the Schwann cell protein gliomedin to neuronal $\mathrm{Nfasc}^{\mathrm{NF} 186}$ allows for the recruitment and stabilization of other nodal proteins (Eshed et al., 2005; Lambert et al., 1997; Schafer et al., 2006). Knockdown of gliomedin resulted in disrupted clustering of nodal proteins, including $\mathrm{Nav}$ channels and $\mathrm{Nfasc}^{\mathrm{NF186}}$ (Dzhashiashvili et al., 2007). Interestingly, one study found that ectopic gliomedin clusters could form along internodes of cultured Schwann cell-myelinated dorsal root ganglion (DRG) neurons when the extracellular domain of $\mathrm{Nfasc}^{\mathrm{NF186}}$ was added to the media (Eshed et al., 2005). The extracellular domain of $\mathrm{Nfasc} \mathrm{NF}^{\mathrm{N} 186}$ contains six immunoglobulin domains, three

fibronectin type III repeats, and a mucin domain (Thaxton and Bhat, 2009). $\mathrm{Nfasc}^{\mathrm{NF186}}$ is then able to recruit AnkG to the nodal domain through its FIGQY motif (Garver et al., 1997). $\mathrm{Na}_{\mathrm{V}}$ channels in turn can bind to AnkG and their $\beta$-subunit can also bind to Nfasc (Brechet et al., 2008; Lemaillet et al., 2003; Malhotra et al., 2000; Rasband, 2008). The 
role for Nfasc ${ }^{\mathrm{NF} 186}$ at PNS nodes has been further verified by in vitro knockdown studies that show loss of Nfasc ${ }^{\mathrm{NF} 186}$ disrupts $\mathrm{Na}_{\mathrm{V}}$ channel clustering at the node (Dzhashiashvili et al., 2007). This study also showed that the external domain of $\mathrm{Nfasc}^{\mathrm{NF} 186}$ is required for its role in nodal organization and $\mathrm{Na}_{\mathrm{v}}$ channel accumulation, reiterating the likely role of glia in nodal organization. In addition, knockdown of $\mathrm{Nfasc}^{\mathrm{NF} 186}$ cannot be rescued by $\mathrm{Nfasc}^{\mathrm{NF} 186}$ missing its AnkG-binding domain, reiterating its role for the recruitment of AnkG and subsequently Nav channels (Dzhashiashvili et al., 2007).

While the organization of the PNS node is generally agreed upon, the formation and stabilization of CNS nodes is not as well understood. Perinodal astrocytes in the CNS are the proposed equivalent of Schwann cell microvilli in the PNS. Perinodal astrocytes fill the extracellular space adjacent to CNS nodes and are thought to interact with nodal proteins (Black and Waxman, 1988; Hildebrand et al., 1993). However, a CNS equivalent of gliomedin has yet to be found. One candidate for this role is Brevican, an ECM protein that localizes to the nodal region and is critical for organizing the CNS nodal ECM (Bekku et al., 2009). Ablation of Brevican in mouse did not reveal major deficits in nodal organization (Brakebusch et al., 2002), but it is possible that other proteins function with Brevican to organize the node and the other components compensate in the knockout. In addition, oligodendrocytes secrete proteins important for clustering nodal proteins prior to the start of myelination (Kaplan et al., 2001). More research is needed to determine the extent to which each of these contributions is needed for CNS node formation.

Previous studies have examined the role of several nodal proteins in the organization and stabilization of the CNS node. For example, deficiencies in $\beta I V$-spectrin resulted in alterations to axon shape, namely in vesicle-filled membrane protrusions in 
the nodal area (Yang et al., 2004). In addition, loss of $\beta$ IV-spectrin decreases the levels of AnkG and $\mathrm{Na}_{\mathrm{V}}$ channels at the node, resulting in disrupted axonal conduction and suggesting it is important for stabilizing this complex (Komada and Soriano, 2002; Lacas-Gervais et al., 2004). Nfasc null mice show disrupted PNS and CNS nodes, but they die at P6, when the nervous system is not fully developed (Sherman et al., 2005). These Nfasc null mice lack both major Nfasc isoforms that are expressed in the nervous system, namely Nfasc ${ }^{\mathrm{NF} 155}$ that is expressed at the paranode of myelinating glia and $\mathrm{Nfasc}^{\mathrm{NF} 186}$ that is expressed at the node and AIS. Loss of both isoforms results in complete disorganization of both paranodes and nodes, which is fatal. To overcome this problem, one group utilized transgenic $\mathrm{Nfasc}^{\mathrm{NF} 186}$ expression in the $\mathrm{Nfasc}$ null mice and found that AnkG and Nav1.6 were able to localize to the node, independent of paranode formation (Zonta et al., 2008). Importantly, this same group found that transgenic rescue of the glial Nfasc isoform, $\mathrm{Nfasc} \mathrm{NF155}^{\mathrm{n}}$, which is localized to the paranode, also rescued nodal components in the CNS (Zonta et al., 2008). This controversial result suggests that formation of the paranode is responsible for formation of the CNS node. However, this contradicts the fact that the node remains intact in paranodal mutant mice, including Caspr, Contactin and Nfasc ${ }^{N F 155}$ null mice (Bhat et al., 2001; Boyle et al., 2001; Pillai et al., 2009). In addition, freeze-fracture electron microscopy (EM) studies showed that early nodal differentiation takes place before paranodal AGSJ formation (Tao-Cheng and Rosenbluth, 1983). Contributing to the debate, more recent work showed that with in vitro co-cultures of Schwann cells and DRGs from wild type and Nfasc null mice, paranodes are responsible for $\mathrm{Na}_{\mathrm{V}}$ channel clustering at mature nodes, independent of Nfasc $^{\mathrm{NF} 186}$ (Feinberg et al., 2010). However, these studies were completed in vitro, which 
is lacking many of the environmental cues that may be critical for nodal organization in vivo. Further support for a paranodal role in node stabilization comes from studies that show loss of AGSJs results in destabilization of $\mathrm{Na}_{\mathrm{v}}$ channels (Ishibashi et al., 2002; Poliak and Peles, 2003; Rios et al., 2003; Rosenbluth et al., 2003). To definitively answer this question, a study needed to be completed in which the node was disorganized while the paranodes remained completely intact.

Recent work from our lab finally addressed this issue in vivo by examining a conditional neuronal knockout of $N f a s c$, so that the organization of the node could be examined developmentally in the absence of $\mathrm{Nfasc}^{\mathrm{NF} 186}$ (Thaxton et al., 2011). In this study, loss of $\mathrm{Nfasc}^{\mathrm{NF} 186}$ resulted in the disruption of nodal clustering, while paranodal and juxtaparanodal domains formed normally. This work showed that $\mathrm{Nfasc}^{\mathrm{NF186}}$ is required for the organization of the PNS and CNS node, independent of paranodes, and paranodes are not sufficient for nodal coordination. In addition, this study reiterates that $\mathrm{Nfasc}^{\mathrm{NF1} 186}$ plays a much different role at the AIS than at the node, in that nodal loss of $\mathrm{Nfasc}^{\mathrm{NF186}}$ also disrupts clustering of AnkG, NrCAM, and the PNS-specific proteins gliomedin and ezrin binding protein 50 (EBP50) (Thaxton et al., 2011). This agrees with previous results that show $\mathrm{Nfasc}^{\mathrm{NF186}}$ can localize to the nodes independently of its interaction with AnkG and that it arrives at the presumptive node prior to other nodal components (Dzhashiashvili et al., 2007; Koticha et al., 2006; Lambert et al., 1997; Lustig et al., 2001; Thaxton et al., 2011). Further, a recent study showed that the fibronectin type-III domain of $\mathrm{Nfasc}^{\mathrm{NF} 186}$ is required for its interaction with gliomedin and thus clustering at the node (Labasque et al., 2011). In addition, $\mathrm{Nfasc}^{\mathrm{NF} 186}$ can interact directly with the $\beta$-subunit of $\mathrm{Nav}_{\mathrm{v}}$ channels, allowing them to be clustered by 
$\mathrm{Nfasc}^{\mathrm{NF} 186}$ at the node (Ratcliffe et al., 2001). Though it is clear that $\mathrm{Nfasc}^{\mathrm{NF} 186}$ is critical for organizing the node, the mechanism of node formation is still elusive. A few pieces of the mechanistic puzzle have begun to materialize based on recent work in which the authors of one study transected axons and removed them from their soma before inducing myelination in vitro (Zhang et al., 2012). The researchers then looked to see which nodal components were able to cluster, thus determining if each component was already localized to the axonal membrane, or needed to be transported from the soma. They found that CAMs were already expressed on the axolemma surface and just needed to be trapped by Schwann cell ligands during myelination to begin to accumulate at the node.

On the other hand, ion channels and cytoskeletal components of the node require transport from the soma to become targeted at the node. In addition, the study found that the recycling of nodal components in mature nodes requires transport from the soma. Together, these data suggest that $\mathrm{Nfasc}^{\mathrm{NF} 186}$ interacts with the extracellular environment or myelinating glial cells to become clustered at the node and this signals for the clustering of other components being trafficked along the axon.

\subsubsection{The node is a barrier against invading paranodes}

A well-known function of the paranode is to act as a barrier for the potassium channels in the JXP to remain separated from the sodium channels at the node (see below for more details). And as already mentioned, the AIS is thought to function as a fence to keep somato-dendritic proteins out of the axon. It should come as no surprise then, that recent work from our lab found a role for the node as a barrier to prevent neighboring paranodes from invading in on each other (Thaxton et al., 2011). When $\mathrm{Nfasc}^{\mathrm{NF} 186}$ was 
conditionally ablated from neurons early in development, the node failed to organize and flanking paranodes moved in on each other. EM studies revealed that paranodal loops that usually abut the nodal space had moved in and overlapped each other, covering the nodal gap (Thaxton et al., 2011). Without this nodal gap, saltatory conduction is disrupted, as $\mathrm{Na}_{\mathrm{V}}$ channel current cannot pass from the extracellular environment.

\subsubsection{The node in disease and injury}

Normal nodal function is critical for action potential propagation and neuronal communication. One only needs to look at the phenotype of mice with disrupted nodes to understand how disruption affects the motor system of mice, let alone other physiological processes (Thaxton et al., 2011). However, the developmental organization of the node is not the only time to worry about node organization. Nodes are also destroyed in many disease states, including multiple sclerosis (MS). In MS, sodium channels clusters are no longer stable at the nodes (Coman et al., 2006; Craner et al., 2004). This disruption of sodium channels is thought to contribute to the pathology of axonal degeneration, which results in loss of function in MS patients (Craner et al., 2004). The mechanisms responsible for disruption of nodal sodium channels are not well understood. However, auto-antibodies have been discovered to Nfasc in MS patients (Mathey et al., 2007). Studies have also been completed in in vitro co-cultures of DRGs and Schwann cells that found addition of antibodies that block the function of CAMs resulted in loss of $\mathrm{Na}_{\mathrm{V}}$ channels and AnkG accumulation at the nodes (Lustig et al., 2001). Therefore, it is possible that in MS patients the auto-antibodies are preventing $\mathrm{Nfasc}^{\mathrm{NF} 186}$ from functioning properly at the node, resulting in destabilization of $\mathrm{Na}_{\mathrm{V}}$ channels from this 
domain. In other autoimmune disorders, including acute motor axonal neuropathy

(AMAN), the nodes are specifically altered and this leads to disruption in action potential propagation (Lonigro and Devaux, 2009; Susuki et al., 2007). Further, in one study the authors injected autoantibodies found in patients with AMAN into rats and found that nodal and paranodal proteins were disrupted and axonal conductance was severely altered (Susuki et al., 2011). This disruption in conduction eventually led to axonal degeneration. Therefore, it is important to definitively understand how the node is organized and maintained so that treatments can begin to be elucidated for these devastating disorders.

\subsection{The paranode}

As myelinating glia wrap around the axon, the cytoplasm of the glial cell is pushed to the edges, forming loops, which then attach to the axolemma (Fig. 1.1). These loops are known as paranodal loops and they attach to the axolemma adjacent to the node through the formation of AGSJs (Salzer, 2003; Thaxton and Bhat, 2009). The AGSJs are very similar to the invertebrate septate junctions that form between ensheathing glial cells and axon bundles of the invertebrate nervous system (Banerjee et al., 2006b; Rosenbluth, 1995). AGSJs are electron-dense structures that form a ladder-like distribution along the membrane of the glial paranodal loops and the axonal membrane (Fig. 1.5) (Schnapp et al., 1976; Wood, 1959). The AGSJs are made by three known components, including Contactin-associated protein (Caspr) and Contactin (Cont) on the axonal side, which bind to each other in $c i s$, and the $155 \mathrm{kDa}$ isoform of Nfasc, $\mathrm{Nfasc} \mathrm{N}^{\mathrm{NF} 155}$ on the glial side (Table 1.1; Fig. 1.5) (Charles et al., 2002; Einheber et al., 1997; Menegoz et al., 1997; Peles et al., 1997; Rios et al., 2000; Tait et al., 2000). Loss of any of these three components 
results in disruption of the AGSJs (Bhat et al., 2001; Boyle et al., 2001; Pillai et al., 2009). Cont is a Glycosylphosphatidylinositol- (GPI-) anchored protein and Caspr is a transmembrane protein, so the AGSJs communicate with the axon through interactions with the Caspr c-terminus (Bhat, 2003). Within the axon at the paranode there is an accumulation of several cytoskeletal scaffolding proteins, including $\alpha$ II- and $\beta$ II-spectrin, protein 4.1B and the adaptor protein AnkB (Table 1.1) (Garcia-Fresco et al., 2006; Ogawa et al., 2006; Ohara et al., 2000; Thaxton and Bhat, 2009). The c-terminus of Caspr contains a 4.1/ezrin/radixin/moesin (FERM) binding domain, which allows it to interact with protein 4.1B (Denisenko-Nehrbass et al., 2003; Gollan et al., 2002; Menegoz et al., 1997; Peles et al., 1997; Poliak et al., 1999). Caspr has been shown to interact in a complex with 4.1B, spectrin and actin, suggesting the AGSJs anchor the paranodal loops to the axonal cytoskeleton (Buttermore et al., 2011; Garcia-Fresco et al., 2006).

The paranode was first viewed as a fence, preventing the mixing of ion channels in the JXP to the ion channels in the node, in the frog brain (Rosenbluth, 1976). More recent studies have conclusively shown that the formation of the AGSJs is critical for potassium channels maintaining their localization to the JXP (Fig. 1.5) (Bhat et al., 2001; Boyle et al., 2001; Dupree et al., 1999; Poliak et al., 2001). Some have called the paranode the Achilles' heel of the axon because it is an area of transition along the axonal microtubules and that can lead to problems with axonal transport and eventually axonal degeneration (Sousa and Bhat, 2007). Though no functional clustering of ion channels exists at the paranode, the function of the paranode as a fence is a critical determinant of axonal function that must be maintained and therefore it is important to understand how the paranode is formed and stabilized. 


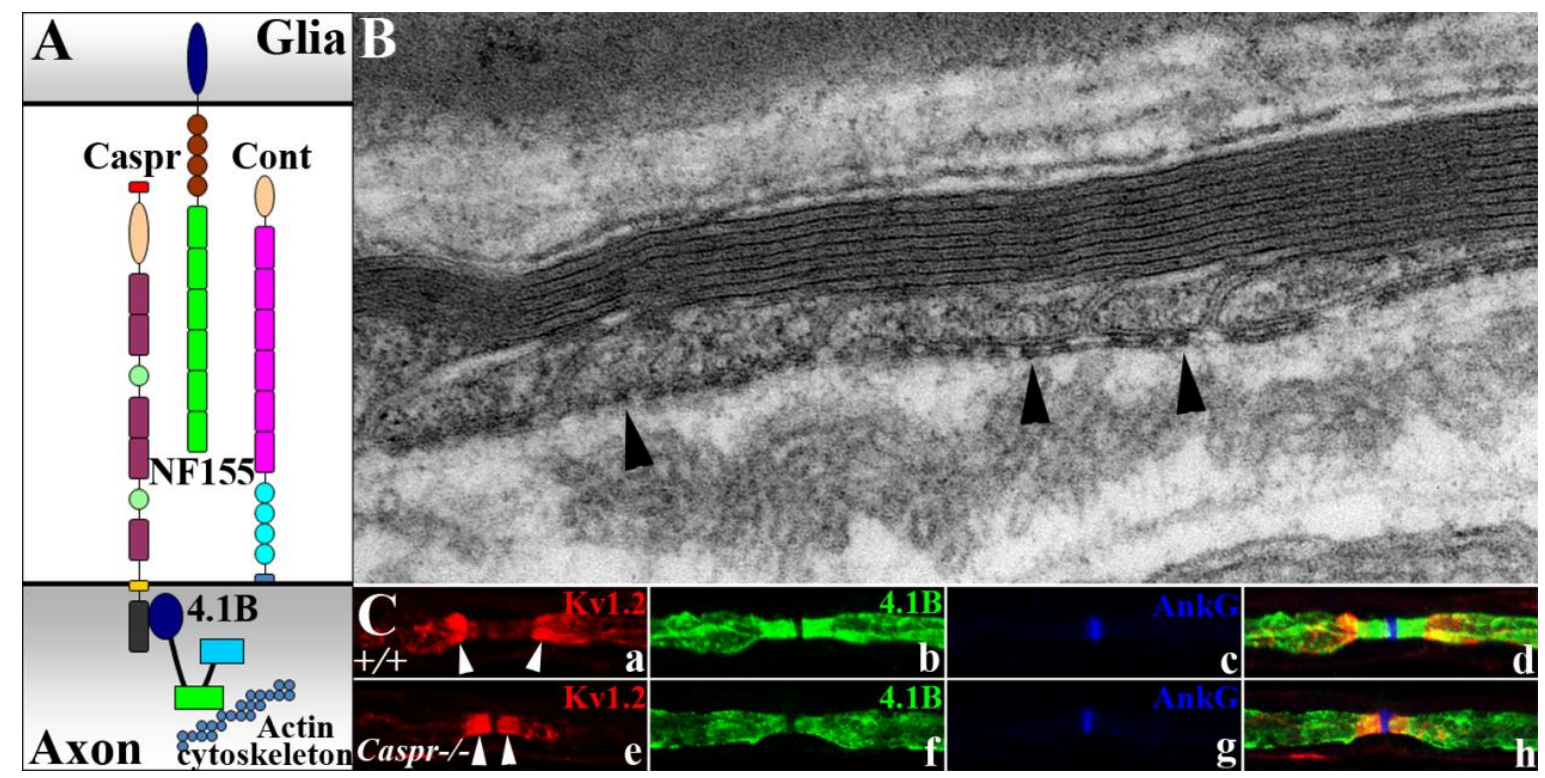

Figure 1.5 Molecular organization of the paranode and AGSJs

(A) Schematic of paranodal domain highlighting the AGSJ components (Caspr, NF155, and Cont) along with protein 4.1B.

(B) Electron micrograph of P30 WT spinal cord at the paranode. The paranodal loops attach to the axonal membrane through the electron-dense AGSJs (arrowheads).

(C) Immunostaining of wild-type (+/+; a-d) and Caspr-/- (e-h) sciatic nerve fibers with antibodies for $\mathrm{K}_{\mathrm{V}} 1.2$ (red), 4.1B (green), and AnkG (blue). This immunostaining highlights the importance of the paranodal AGSJs for segregation of potassium channels to the JXP $\left(\mathrm{K}_{\mathrm{V}} 1.2\right.$, a, arrowheads). In the Caspr-/- the potassium channels become localized next to the node ( $\mathrm{K}_{\mathrm{V}} 1.2$, e, arrowheads). 


\subsubsection{Formation of the paranode}

While the clustering of nodal proteins requires interactions with external cues, and the clustering of AIS proteins does not, the contribution from external sources in the organization of the paranode is a little less clear. An in vitro study showed that Caspr must form a complex with Cont to be transported from the endoplasmic reticulum to the plasma membrane (Faivre-Sarrailh et al., 2000). On the other hand, ablation of Caspr, or deletion of the Caspr c-terminus, also leads to the loss of Cont from the paranode (Bhat et al., 2001; Gollan et al., 2002). Further, in the absence of Caspr, Cont localizes to CNS nodes (Rios et al., 2000). Thus, Caspr and Cont are interdependent for stable localization at the paranode. However, it is not clear whether $\mathrm{Nfasc}^{\mathrm{NF155}}$ pulls Caspr/Cont to the paranode, or whether the Caspr/Cont complex stabilizes the paranodal loops. Glialspecific ablation of $\mathrm{Nfasc}^{\mathrm{NFI55}}$ also results in loss of AGSJs and paranodal organization (Pillai et al., 2009), so Caspr/Cont can't form paranodes in the absence of $\mathrm{Nfasc}^{\mathrm{NF155}}$.

\subsubsection{Role of axonal cytoskeleton in axo-glial septate junction (AGSJ) organization}

Several mechanisms have been proposed for paranodal organization. It was previously not clear whether the paranodal cytoskeletal components recruit the AGSJ to the paranodal membrane, or whether they function only to stabilize the complex. The cytoskeletal protein 4.1B becomes diffusely localized along the axon in paranodal mutants, suggesting 4.1B requires its interaction with Caspr and the AGSJs to become enriched at the paranode (Fig. 1.5) (Gollan et al., 2002). In addition, recent work shows that the paranodal proteins Caspr and Cont are able to localize to the paranode, but do not remain stabilized there, in the absence of protein 4.1B (Buttermore et al., 2011; 
Cifuentes-Diaz et al., 2011). When 4.1B was lost, the paranodes became broken and EM showed that the electron-dense AGSJs were destabilized and eventually lost, resulting in the pulling off of paranodal loops from the axolemma (Buttermore et al., 2011). These data support earlier findings that Caspr forms a complex with the axonal cytoskeleton through its interactions with protein 4.1B, $\alpha$ II-spectrin, $\beta$ II-spectrin, AnkB and actin (Denisenko-Nehrbass et al., 2003; Garcia-Fresco et al., 2006; Gollan et al., 2002; Ogawa et al., 2006). This suggests that the AGSJs form in the absence of their interaction with the axonal cytoskeleton, but must remain attached for long-term stabilization.

\subsubsection{Role of lipid rafts in AGSJ organization}

While the complex that links Caspr to the axonal cytoskeleton is beginning to be elucidated, the scaffolding components within the glial paranodal loops that stabilize $\mathrm{Nfasc}^{\mathrm{NF} 155}$ at the paranode are not known. It is possible that lipid rafts play a role in this

organization during myelination (Simons et al., 2000). In addition to $\mathrm{Nfasc}^{\mathrm{NF} 155}$, several proteins are critical for proper AGSJ formation, including ceramide galactosyltransferase (CGT), proteolipid protein (PLP), myelin-basic protein (MBP), myelin-associated glycoprotein (MAG), 2',3'-cyclic nucleotide 3'-phosphodiesterase and Nkx6-2 (Coetzee et al., 1996a; Garcia-Fresco et al., 2006; Honke et al., 2002; Klugmann et al., 1997; Lappe-Siefke et al., 2003; Rasband et al., 2005; Rosenbluth, 1981; Southwood et al., 2004; Trapp et al., 1989). CGT is an enzyme that helps synthesize the myelin lipids galactocerebroside and sulfatide (Coetzee et al., 1996b; Dupree et al., 1999; Dupree and Popko, 1999; Marcus et al., 2002; Marcus et al., 2006). Ablation of CGT resulted in disruption of AGSJs and loss of the segregation of nodal sodium channels and 
juxtaparanodal potassium channels (Dupree et al., 1999; Marcus et al., 2002). These data, along with subcellular fractionation studies of $\mathrm{Nfasc}^{\mathrm{NF} 155}$, help to solidify the idea that lipid rafts form in the paranodal loops and help organize the glial paranodal components to stabilize $\mathrm{Nfasc}^{\mathrm{NF} 155}$ (Schafer et al., 2004). It is clear from all of these data that the paranode organizes through a coordinated effort of glial and neuronal signals, each required for the proper establishment of the AGSJs.

\subsubsection{The paranode is a fence against the invading juxtaparanodal complex}

As previously mentioned, the role of the paranode is to provide a fence that keeps the potassium channels in the JXP separate from the sodium channels in the node (Fig. 1.5). Loss of any AGSJ component results in dissolution of the AGSJs (Bhat et al., 2001; Boyle et al., 2001; Pillai et al., 2009). When the AGSJs are lost, the gap between the glial paranodal loop membrane and the axolemma widens. However, the paranodal loops do not completely lift away from the axonal membrane causing unraveling of the myelin because other adhesive complexes and extracellular support help maintain the myelin. For example, the PNS basal lamina may help maintain the myelin in the absence of AGSJs (Poliak and Peles, 2003). However, loss of the AGSJs has grave consequences for axonal conductance and axonal survival. Mice that have lost Caspr do not live past 30 days and have severe deficits in axonal conduction velocity and amplitude (Bhat et al., 2001). This study revealed that the hyperpolarization waveform of the action potential is also disrupted in Caspr null sciatic nerves. These disruptions are likely due to the loss of segregation of potassium channels at the JXP, as seen in all paranodal mutants (Fig. 1.5) (Bhat et al., 2001; Dupree et al., 1999; Garcia-Fresco et al., 2006; Pillai et al., 2009). 
In addition to its role as a fence to separate nodal sodium channels and juxtaparanodal potassium channels, the paranodal complexes are also critical for maintaining the organization of the axonal cytoskeleton. Further examination of the Caspr and Nfasc ${ }^{N F 155}$ null axonal organization reveals the presence of swellings along the axons (Garcia-Fresco et al., 2006; Pillai et al., 2009). EM of these swellings revealed complete disorganization of the normally parallel arrays of axonal cytoskeleton. In addition, organelle accumulation was found in the paranodal region flanking these large swellings, a sign of disrupted axonal transport that eventually leads to axon degeneration (Garcia-Fresco et al., 2006). The paranodal region is highly susceptible to disrupted axonal transport (Sousa and Bhat, 2007). The swellings that result from disrupted domain organization also contain increased levels of phosphorylated neurofilaments, a sign that the cytoskeleton is disrupted (Pillai et al., 2009). Importantly, the presence of swellings and cytoskeletal abnormalities are found prior to axonal degeneration in many neuropathies, suggesting a common mechanism of neuronal destruction in these pathologies (Fabrizi et al., 2007; Lappe-Siefke et al., 2003; Rodriguez and Scheithauer, 1994). And in Purkinje neurons, the accumulation of organelles is an indicator of axonal degeneration (Palay and Palay, 1974). These studies also show that the function of the link between the AGSJs and the axonal cytoskeleton is not just to anchor the paranodal loops, but also to organize and stabilize the axonal cytoskeleton. And this function of the AGSJs is critical for maintaining axonal health and stability. 


\subsubsection{The paranode in disease}

Demyelination is a major contributor to disease progression and axonal degeneration in diseases such as Charcot-Marie Tooth (CMT) disease and MS. These disorders result from various causes, including genetic mutations of a Connexin isoform in CMT disease and autoimmune disruption in MS, but both result in axonal domain disorganization (Berger et al., 2006; Lubetzki et al., 2005; Nave et al., 2007; Oguievetskaia et al., 2005; Shy, 2006; Trapp and Nave, 2008). In addition to the disruption of nodes, MS patients also present with disrupted paranodal organization, as shown by loss of Caspr enrichment and disrupted potassium channel localization (Coman et al., 2006; Howell et al., 2006; Wolswijk and Balesar, 2003). Nfasc ${ }^{\mathrm{NF} 155}$ levels are also decreased at the paranodes of MS patients, with decreased lipid raft-association (Maier et al., 2007; Maier et al., 2005). Destabilization of these paranodal proteins resulted in disruption of the AGSJs, as shown by the movement of potassium channels into the paranodal region (Howell et al., 2006). As previously mentioned, auto-antibodies to Nfasc are found in MS patients and may play a role in the disruption of this protein at the node (Nfasc ${ }^{\mathrm{NF186}}$ ) and paranode (Nfasc ${ }^{\mathrm{NF155}}$ ) (Mathey et al., 2007). Further, axonal swellings that result from disorganization of axonal domains are also found in neurodegenerative diseases, including amyotrophic lateral sclerosis (ALS), CMT, Wallerian degeneration, Alzheimer's disease and cerebrospinal ataxia (Brownlees et al., 2002; Collard et al., 1995; Stokin et al., 2005). Based on the swellings found in these diseases and the swellings seen at the paranodes of AGSJ mutant mice, this may represent a conserved response to axonal distress and an early sign of axonal degeneration. In the early stages of ALS, the AIS diameter is increased (Sasaki and 
Maruyama, 1992), so this may be a conserved mechanism of a diseased axon. Each of these studies points to the disruption of axonal domains as part of the pathology that leads to demyelination and axonal degeneration. Therefore, future studies should be focused on trying to rebuild the lost paranodal interactions.

\subsection{The juxtaparanode (JXP)}

The JXP flanks the paranode and delayed rectifier potassium channels, including $\mathrm{K}_{\mathrm{V}} 1.1$ and $\mathrm{K}_{\mathrm{V}} 1.2$, are enriched at the JXP (Fig. 1.1; Table 1.1; Fig. 1.6) (Rhodes et al., 1997; Wang et al., 1993). Potassium channels form a complex with the axonal, transmembrane CAM Caspr2 at the JXP (Fig. 1.6) (Poliak et al., 1999). In addition, the GPI-anchored CAM transient axonal glycoprotein (Tag1) is localized to both the glial and axonal membranes at the JXP, forming a complex with Caspr2 (Table 1.1; Fig. 1.6) (Poliak et al., 2003; Traka et al., 2002; Traka et al., 2003). Compact myelin is required for potassium channel localization and stabilization at the JXP (Baba et al., 1999).

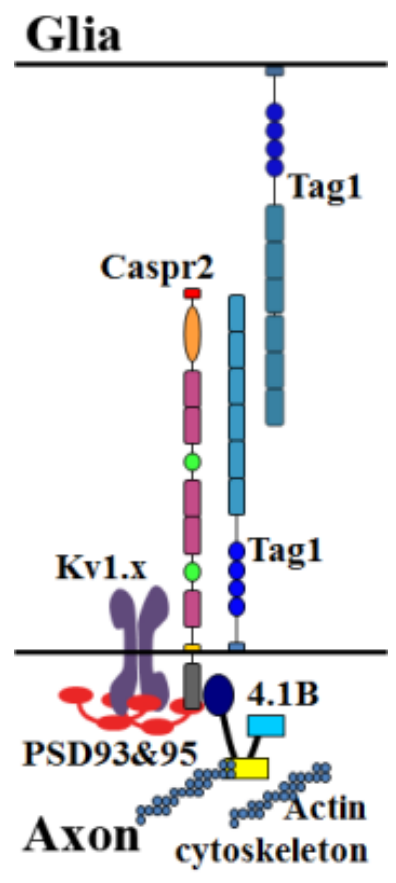

Figure 1.6 Molecular organization of the JXP

Schematic of the JXP domain that highlights the key molecular components that are critical for JXP organization and function. 


\subsubsection{Role of axonal cytoskeleton in JXP organization}

Importantly, individual loss of Caspr2 and Tag1 each result in loss of potassium channel clustering at the JXP (Poliak et al., 2003). Within the axon at the JXP there are several post-synaptic density scaffolding proteins, including PSD93 and PSD95 (Baba et al., 1999; Horresh et al., 2008). However, ablation of these scaffolding proteins did not disrupt the localization of potassium channels to this domain, or disrupt binding of Caspr2 with potassium channels (Horresh et al., 2008; Rasband et al., 2002).

Interestingly, this is in contrast with the role for PSD proteins at the AIS, in that PSD93, but not Caspr2 or Tag1, are required for clustering of potassium channels at the AIS (Ogawa et al., 2008). Further, the Caspr2 c-terminus contains a region, just like Caspr, that mediates its binding to protein 4.1B (Denisenko-Nehrbass et al., 2003; Gollan et al., 2002; Menegoz et al., 1997; Peles et al., 1997; Poliak et al., 1999). In one study, the authors used GST-fusion proteins of the Caspr2 c-terminus with mutations in the PDZbinding motif and in the 4.1-binding domain and found that only Caspr2 lacking its 4.1 binding domain was unable to interact with the membrane associated guanylate kinases, suggesting that the interaction with 4.1 may be more important for JXP organization (Horresh et al., 2008). Protein 4.1B is enriched at the JXP, as it is at the paranode, and loss of 4.1B results is diffusion of potassium channels, Caspr2, and PSD95 from the JXP (Buttermore et al., 2011; Cifuentes-Diaz et al., 2011; Horresh et al., 2010). While 4.1B is required for the clustering of JXP components, it is not required to maintain the interaction between Caspr2 and $\mathrm{K}_{\mathrm{V}} 1$ channels (Buttermore et al., 2011). 


\subsubsection{Role of JXP potassium channels in action potential propagation}

As previously described, the localization of potassium channels to the JXP also requires the proper formation of the paranode, as disrupted paranodes result in movement of potassium channels to sit next to the node (Bhat et al., 2001; Boyle et al., 2001;

Garcia-Fresco et al., 2006; Pillai et al., 2009). This means that the segregation of potassium channels involves sorting mechanisms at the paranodal axolemma to separate the $\mathrm{K}_{\mathrm{V}} 1$ channels and JXP CAMs from the CAMs at the paranode, then the formation of complexes to anchor them properly with the underlying cytoskeleton (Poliak and Peles, 2003). The importance of segregating the potassium channels to the JXP lies in the role potassium channels play in the action potential. Delayed rectifier potassium channels are responsible for the repolarization event that brings the membrane potential back down after it has been depolarized (Benatar, 2000). Additionally, the potassium channels bring the membrane potential a bit past the resting membrane potential, in a hyperpolarized state, that helps prevent backpropagation of action potentials (Purves et al., 2004). While disrupted potassium channel localization does not disrupt conduction velocity of action potentials, it does make the nerve more hyperexcitable (Cifuentes-Diaz et al., 2011). When potassium channels were mislocalized in $4.1 B$ null mice, spontaneous and evoke repetitive activity was found in knockout, but not control axon fibers, a sign up hyperactivity (Cifuentes-Diaz et al., 2011). These results agree with previous studies that show potassium channels help modulate neuronal activity and disruption of potassium channel function leads to neuronal hyperexcitability and in some cases, disorders such as epilepsy (Lopantsev et al., 2003; Sutherland et al., 1999; Watanabe et al., 2000). Together, these studies underlie the importance of maintaining axonal domain 
organization for the proper balance of neuronal activity levels. When any one domain is disrupted, the normal neuronal firing properties are altered and this can have devastating consequences.

\subsection{The internode}

While the internode is most well known as the area of tightly wrapped myelin, it is not a region devoid of axon-glial interactions. The myelin is so compact at the internode that the space between the axon and the membrane is only about $12-13 \mathrm{~nm}$ (Maurel et al., 2007). Interestingly, the link between the myelin and the axon at the internode was disrupted by proteases, suggesting that there are transmembrane proteins localized to this area that help to stabilize the interaction (Maurel et al., 2007; Yu and Bunge, 1975). Also, along the internode of mature myelinating glial cells, there are areas of cytoplasm channels, known as Schmidt-Lanterman incisures, where links form between the adaxonal membrane (the glial membrane that contacts the axon) and the abaxonal membrane (the glial membrane that contacts the ECM) (Poliak and Peles, 2003). The proposed role of the incisures is in transport of materials across the myelin to allow for maintenance of the myelin and growth, if needed (Arroyo et al., 2001; Ghabriel and Allt, 1981). In order for these structures to compact and organize properly, there must be cell surface proteins responsible for communicating between to the two cells.

A few proteins have been discovered that are localized to the internode and/or incisures and could play a role in their organization. First, the CAM MAG is localized to Schmidt-Lanterman incisures, as well as along the internode (Trapp, 1990; Trapp and Quarles, 1984; Trapp et al., 1984). Since MAG is an Ig superfamily CAM, like Caspr and 
Caspr2, it could play a role in axo-glial interactions at the internode (Lai et al., 1987; Salzer et al., 1987). Studies have shown that MAG mutant mice have normal myelination, but have small alterations along the internodal space between the myelin and axon (Li et al., 1994; Montag et al., 1994). The other proteins that are also localized to the myelin-axon interface at the internode include several transmembrane Nectin-like (Necl) proteins (Maurel et al., 2007; Sakisaka and Takai, 2004). Similar to other CAMs involved in axo-glial interactions, including Caspr and Caspr2, the Necl proteins belong to the Ig- superfamily and contain binding domains for 4.1 proteins and PDZ binding motifs (Ogita and Takai, 2006; Takai et al., 2003). Interestingly, Necl-2 has been shown to function in adhesion both homophilically and heterophilically, and can interact with a truncated form of protein 4.1B (Biederer et al., 2002; Masuda et al., 2002; Shingai et al., 2003; Yageta et al., 2002). In the PNS, Necl-1 and Nec1-2 are expressed within the axon and Necl-4 and Necl-2 are expressed within Schwann cells (Maurel et al., 2007).

Immunohistochemical and binding analyses revealed that along the internode, Necl-4 and Necl-1 interact to help maintain a stable interaction (Maurel et al., 2007). In addition, at the Schmidt-Lanterman incisures, several homophilic and heterophilic interactions occur between the various Necl proteins (Maurel et al., 2007).

Interestingly, a recent paper found protein $4.1 \mathrm{G}$ is a novel component of internode organization (Ivanovic et al., 2012). 4.1G is expressed in Schwann cells and was shown to colocalize with Necl-4 (Horresh et al., 2010; Ivanovic et al., 2012). Interestingly, in $4.1 G^{-/}$sciatic nerve fibers, Necl-4 failed to properly cluster at the Schmidt-Lanterman incisures (Ivanovic et al., 2012). Further, the authors found that in the absence of 4.1G, paranodal proteins appeared in broken segments adjacent to the paranode and JXP 
components, such as $\mathrm{K}_{\mathrm{V}} 1.2$ channels, accumulated by the broken paranodal segments and were no longer properly localized along the inner mesaxon. This study reiterates the fact that there are still other molecular components yet to be discovered that play critical roles in the organization and maintenance of axonal domains, including the internode.

In addition to adhesion between the axon and myelinating glia, the myelin must sense signals from the axon for proper myelination. For example, during development the axon diameter must be read by the myelinating glial cell so that the proper number of myelin wraps is achieved (Smith et al., 1982). At the completion of this process, there is a conserved ratio of axon diameter and myelin thickness, known as the g ratio.

Interestingly, the g ratio is determined differently by Schwann cells and oligodendrocytes. In the PNS, but not CNS, myelin thickness is disrupted in mice with altered levels of phosphorylated neurofilaments, which help organize the axon cytoskeleton (Elder et al., 2001). Studies have also shown that the g ratio can be disrupted by altering expression of Neuregulin 1 (Nrg1), revealing an interaction between the glial protein tyrosine kinase receptor ErbB2 and Nrg1 in myelin wrapping (Michailov et al., 2004; Sousa and Bhat, 2007). These data reveal that although the myelin is very compactly wrapped around the internode, CAMs and their binding partners still play an important role in axo-glial interactions at the internode.

\subsection{Conclusions and an evolutionary perspective}

Myelin proteins and the need to insulate axons evolved separately several times, including in Annelids, Arthropods and Chordates, highlighting the importance of its function for efficient neuronal transmission (Hartline, 2008; Roots, 2008). Interestingly, 
the mechanisms responsible for clustering ion channels at axonal domains evolved prior to myelination (Hill et al., 2008). For example, the structural amino acid motif in sodium channels that allows them to link to the actin-spectrin cytoskeleton through AnkG evolved in early chordates, allowing for the clustering of sodium channels at the AIS before nodes evolved (Garrido et al., 2003; Hill et al., 2008; Lemaillet et al., 2003; Rasband, 2008). Further, this AnkG binding motif is also conserved in potassium channels that localize to the AIS and node (Pan et al., 2006; Rasband, 2008). However, the potassium channel motif evolved concurrent with myelination, as potassium channels are needed for proper saltatory conduction (Hill et al., 2008). Importantly, nodes are not found in invertebrate nervous systems. There are structures similar to the vertebrate AIS. However, the action potential initiation site in invertebrate axons occurs at variable distances from the soma and one neurite can split into both axon and dendrite distally from the soma (Maniar et al., 2012; Meyrand et al., 1992; Rolls et al., 2007). Interestingly, axonal domains in C. elegans are analogous to the vertebrate AIS, including the clustering of AnkG and microtubule-stabilizing protein UNC-33, which is the C. elegan protein collapsing response mediator protein-2 (CRMP-2) (Maniar et al., 2012). In vertebrates CRMP-2 is important for axon specification (Fukata et al., 2002a; Fukata et al., 2002b; Inagaki et al., 2001; Kimura et al., 2005; Maniar et al., 2012). Thus, the mechanisms responsible for sodium channel clustering and axonal organization appear to be conserved through evolution.

In addition to evolutionarily conserved motifs on AIS and nodal ion channels, the paranodal AGSJs are also high conserved through evolution (Banerjee et al., 2006b). Septate junctions are prominent in the epithelia and nervous system of invertebrates, but 
are only found at the vertebrate AGSJs (Banerjee et al., 2006b). Importantly, the three main components of the AGSJs are highly conserved in invertebrates, such as in Drosophila, where orthologs for Caspr/Cont/Nfasc ${ }^{\mathrm{NF} 155}$ are Neurexin IV/Cont/Neuregulin (Banerjee et al., 2006b; Faivre-Sarrailh et al., 2004). In addition, Neurexin IV contains a 4.1-binding sequence in its c-terminus (Banerjee et al., 2006b; Baumgartner et al., 1996; Bellen et al., 1998; Bhat, 2003; Bhat, 2004) and the 4.1 family ortholog Coracle colocalizes with Neurexin IV at septate junctions (Fehon et al., 1994). Comparison between the mutational studies in vertebrates and invertebrates reveals a common mechanism whereby the junctions are stabilized through their interaction with 4.1 proteins to the underlying cytoskeleton (Baumgartner et al., 1996; Buttermore et al., 2011; Fehon et al., 1994; Horresh et al., 2010; Lamb et al., 1998; Ward et al., 1998). The conserved functions of septate junctions and ion channel clustering across evolution reiterate the importance of axonal domain organization for maintenance of neuronal function. Thus, the more we know about the organization and maintenance of the AIS, node, paranode, JXP and internode, the better chance we have to treat and prevent the disorders that target these domains. And if we can understand how to maintain the fence function of the AIS, node and paranode, then we may be able to restore ion channel localization in demyelination. This knowledge could then also eventually contribute to the prevention of axonal degeneration that often accompanies these disorders. Together, these studies reveal that the ability of neurons to communicate in complex networks involves intricate wiring during development and maintenance of domain segregation throughout the life of each neuron. 


\section{Chapter 2}

\section{The Cytoskeletal Adaptor Protein Band 4.1B is Required for the Maintenance of Paranodal Axo-Glial Septate Junctions in Myelinated Axons}

\subsection{Introduction}

A fundamental characteristic of myelinated axons is their organization into distinct molecular domains that allows for saltatory action potential propagation. These domains include nodes, where voltage-gated sodium channels are enriched; paranodes, where myelin loops establish AGSJs with the axolemma, and JXPs, where delayedrectifier potassium channels are clustered (Bhat, 2003; Salzer, 2003; Thaxton and Bhat, 2009). Genetic ablation of Caspr resulted in loss of paranodal AGSJs and disorganization of the paranodal axonal cytoskeleton (Bhat et al., 2001; Garcia-Fresco et al., 2006). Disruption of paranodal AGSJs in Cgt, Caspr, Cont and Nfasc ${ }^{N F 155}$ mutants permitted juxtaparanodal components to move alongside nodal sodium channels, indicating that AGSJs serve a fence function at paranodes (Bhat et al., 2001; Boyle et al., 2001; Dupree et al., 1999; Pillai et al., 2009). Another member of the Caspr family, Caspr2, is required for the organization of the juxtaparanodal domain and localization of potassium channels (Poliak et al., 2003). The stabilization of Caspr and Caspr2, and their associated complexes, is thought to depend on cytoskeletal adaptor proteins that link these complexes with axonal and glial cytoskeleton for their long-term stability. 
It was previously reported that the extracellular domain of Caspr is sufficient for membrane targeting, while the intracellular domain is required for Caspr stabilization at the paranodes (Gollan et al., 2002). Interestingly, both Caspr and Caspr2 contain 4.1binding sequences, suggesting that their interactions may be physiologically relevant (Denisenko-Nehrbass et al., 2003; Girault et al., 1998). Several members of the 4.1 protein family are expressed in the nervous system (Ohara et al., 2000; Parra et al., 2000; Yamakawa et al., 1999). Among these, only 4.1B is enriched at paranodal and juxtaparanodal domains in myelinated axons (Denisenko-Nehrbass et al., 2003; Ohara et al., 2000). The presence of a FERM and a spectrin-actin binding domain make 4.1B an ideal candidate to link Caspr and Caspr2-dependent complexes with the underlying axonal cytoskeleton. Disruption of paranodal AGSJs in Caspr and Cont mutants alters 4.1B localization (Garcia-Fresco et al., 2006; Gollan et al., 2002). However, Caspr2 mutants showed normal 4.1B localization (Traka et al., 2003). Recent studies showed that loss of 4.1B does not affect paranodal Caspr localization in sciatic nerves; and in vivo deletion of 4.1B-binding region in Caspr and Caspr2 did not affect Caspr localization, but affected Caspr2 localization (Horresh et al., 2010).

Here we report that 4.1B is essential for proper Caspr localization and maintenance of the paranodal and juxtaparanodal domains. We demonstrate that loss of 4.1B leads to destabilization of AGSJs at the paranodes and complete disorganization of the juxtaparanodal complexes. We also show that $4.1 B$ mutants generated previously (Yi et al., 2005) have proper localization of paranodal proteins even after one year in the PNS, and that 4.1R is not involved in paranodal organization or rescue of $4.1 \mathrm{~B}$ mutant paranodes. Together, our results establish an important role of 4.1B as a cytoskeletal 
adaptor protein that links axo-glial junctional complexes with axonal cytoskeleton to ensure long-term stability and maintenance of axonal domains in myelinated axons.

\subsection{Materials and Methods}

\subsubsection{Animals}

All animal experiments were carried out according to UNC-IACUC approved guidelines for ethical treatment of laboratory animals. 4.1B/Epb4.1l3 knockout mice described previously (Yi et al., 2005) and transgenic mice expressing Cre recombinase under the ubiquitous $\beta$-Actin promoter $(\beta$-Actin-Cre) were purchased from Jackson Labs (Maine, USA).

\subsubsection{Generation of 4.1B/Epb4.113 Conditional Mutants}

The 4.1B/Epb4.1l3 locus spans $\sim 133 \mathrm{~kb}$ of genomic DNA and generates several alternatively spliced mRNAs (referwww.ensembl.org/Mus_musculus). To conditionally ablate the $4.1 B$ gene, a targeting construct was generated in which loxP sites were targeted to flank exon 6. Using BAC recombineering technology, a BAC clone $p B A C$ B.6 from $129 A B 2.2$ BAC library containing the $4.1 B$ locus was targeted such that a neo cassette flanked by $F R T$, FRT-loxP sites was inserted in intron 5 and a second loxP site was inserted in intron 6. Prior to ES cell targeting, the functionality of the loxP sites, as well as the expected restriction enzyme digestion patterns in the targeting vector, were confirmed. The targeting construct was linearized with NotI followed by electroporation into embryonic stem (ES) cells. The targeted ES cells were screened by Southern blotting 
with a 500bp probe upstream of the targeting vector in the $4.1 B$ gene. Two independent targeted clones were used for generating 4.1B-floxed mice using standard procedures.

\subsubsection{Generation of 4.1B Antibodies}

cDNA sequences encoding a portion of the U3 domain of 4.1B (amino acids 655793) were PCR amplified from a mouse cDNA library, cloned into $p E T 28(a+)$ vector (Novagen), and expressed in E.coli BL21(DE3). The recombinant protein was purified over a Ni-Sepharose column and used as an antigen to immunize rabbits and guinea pigs, as described previously (Bhat et al., 2001).

\subsubsection{Tissue Preparation, Antibodies and Immunostaining}

The following antisera were previously described: guinea pig and rabbit antiCaspr (Bhat et al., 2001; Pillai et al., 2009; Thaxton et al., 2010), guinea pig anti-NF186 and rat anti-pan Nfasc (Pillai et al., 2009; Thaxton et al., 2010), rat anti-AnkG, rabbit anti-Caspr2 (Thaxton et al., 2010), rabbit polyclonal anti-4.1R and 4.1N (Kang et al., 2009) and rabbit and guinea pig anti-4.1B antibodies (this study). Other primary antibodies used include: mouse anti-potassium channels ( $\mathrm{K}_{\mathrm{v}} 1.1, \mathrm{~K}_{\mathrm{V}} 1.2$, NeuroMab), mouse anti-PSD95 (NeuroMab), and rabbit anti- $\alpha$-Tubulin (Cell Signaling). Secondary antibodies used for immunofluorescence were: Alexa Fluor conjugated -488, -568, and 647 (Molecular Probes, Invitrogen). The horse-radish peroxidase (HRP)-conjugated secondary antibodies were purchased from Jackson Immunologicals.

Tissues used for immunostaining were prepared as previously described (Thaxton et al., 2010). Briefly, sciatic nerves were removed from anesthetized litter-mate wild type 
and mutant mice and fixed in $4 \%$ paraformaldehyde in phosphate buffer saline (PBS) for 30 minutes at $4^{\circ} \mathrm{C}$. The nerves were washed with PBS several times and either stored at $4^{\circ} \mathrm{C}$ or immediately teased. The nerves were teased into individual fibers in PBS, mounted on glass slides, and dried overnight at room temperature, followed by storage at $-80^{\circ} \mathrm{C}$ or immediate processing by treatment with acetone at $-20^{\circ} \mathrm{C}$ for 20 minutes and several washes with PBS, followed by immunostaining. For spinal cord sections, wild type and mutant mice were deeply anesthetized and transcardially perfused with saline buffer followed by ice cold 4\% paraformaldehyde in PBS. The spinal cord was dissected out and post-fixed in $4 \%$ paraformaldehyde overnight at $4{ }^{\circ} \mathrm{C}$. The tissues were rinsed several times in PBS and sectioned to $30 \mu \mathrm{m}$ using a Vibratome (Leica). The spinal cord sections were then immediately immunostained as previously described (Garcia-Fresco et al., 2006; Pillai et al., 2009).

\subsubsection{RNA preparation and RT-PCR}

Dorsal root ganglia (DRG) from $\beta$-Act-Cre $; 4.1 B^{\text {Flox }}, 4.1 B^{-/}$and control littermate mice of either sex were collected and simultaneously processed in RNAlater Stabilization Reagent (Qiagen). Total RNA was isolated using QIAshredder columns (Qiagen) and RNeasy Mini Kit (Qiagen). RNA concentration was estimated and equal amount of RNA were used for reverse transcription followed by PCR amplification using the MyTaq OneStep RT-PCR Kit (Bioline). Primers used for PCR were as follows: 3'; 
4.1R: 5'-AAGAGTTTAGCGGCTGAAGC-3' and 5'-CGGGCTTCTGGGAGGCTT3'; and Actin: 5'- GCTCCGGCATGTGCAA-3' and 5'AGGATCTTCATGAGGTAGT-3’.

\subsubsection{Immunoblotting and Immunoprecipitation}

Sciatic nerves from littermate wild type and mutants of either sex were excised and crushed using a glass homogenizer in ice cold lysis buffer $(50 \mathrm{mM}$ Tris- $\mathrm{HCl}, \mathrm{pH} 7.5$, $150 \mathrm{mM} \mathrm{NaCl}, 10 \mathrm{mM}$ EDTA, $1 \%$ Triton X-100, 1\% SDS, and a protease cocktail tablet). The lysate was incubated on ice for $30 \mathrm{~min}$ and centrifuged at $16,000 \mathrm{x} g$ for $20 \mathrm{~min}$ at $4^{\circ} \mathrm{C}$. The supernatant was used for protein estimation and immunoblotting.

Spinal cords from littermate wild type and mutants of either sex were excised and either directly processed or frozen at $-80^{\circ} \mathrm{C}$. Spinal cords were homogenized using a glass mortar and pestle on ice with $1 \mathrm{ml}$ ice-cold homogenization buffer $(50 \mathrm{mM}$ Tris-HCl, $\mathrm{pH}$ 7.5, $50 \mathrm{mM} \mathrm{NaCl}, 320 \mathrm{mM}$ sucrose, $10 \mathrm{mM}$ EDTA, $\mathrm{pH} 8.0$, and a protease inhibitor cocktail tablet). The homogenate was centrifuged at $1000 \mathrm{x} g$ for 10 minutes at $4^{\circ} \mathrm{C}$. The supernatant was collected and subjected to further centrifugation at $100,000 \mathrm{x} g$ for 30 minutes at $4^{\circ} \mathrm{C}$. The resulting pellet was then re-suspended in lysis buffer $(50 \mathrm{mM}$ Tris$\mathrm{HCl}, \mathrm{pH} 7.5,50 \mathrm{mM} \mathrm{NaCl}, 10 \mathrm{mM}$ EDTA, $1 \%$ Triton $\mathrm{X}-100$, and a protease inhibitor cocktail tablet) and incubated for 30 minutes at $4{ }^{\circ} \mathrm{C}$ with occasional trituration followed by centrifugation at $100,000 \times g$ for 30 minutes at $4^{\circ} \mathrm{C}$ and the supernatant was collected as the final lysate. Protein concentrations of the lysate were determined using the Lowry Assay (BC assay, BioRad). 
For immunoprecipitation (IP), immobilized Protein A-Sepharose beads (Thermo Scientific) were washed with PBS and incubated with either primary antibodies for 2-4 hours at $4^{\circ} \mathrm{C}$ (antibody-bound beads) or $500 \mu \mathrm{g}$ total protein for $1-2$ hours at $4^{\circ} \mathrm{C}$ (preclear beads). The antibody-bound beads were washed once and the lysate from the preclear beads was added and rotated overnight at $4{ }^{\circ} \mathrm{C}$. After brief centrifugation, the supernatant was collected as the post-IP material. The pre-clear and IP beads were washed three times with wash buffer (50mM Tris- $\mathrm{HCl}, \mathrm{pH} 7.5,50 \mathrm{mM} \mathrm{NaCl})$. Lysate, pre-clear beads, IP beads and post-IP material were resolved on SDS-PAGE and transferred onto nitrocellulose membranes, followed by immunoblotting procedures described above.

\subsubsection{Transmission Electron Microscopy (TEM)}

TEM of age-matched wild type, $\beta$-Act-Cre $; 4.1 B^{\text {Flox }}$ and $4.1 B^{-/-}$mutant mice of either sex was carried out essentially as described previously (Garcia-Fresco et al., 2006; Pillai et al., 2009; Thaxton et al., 2010).

\subsubsection{Image analysis}

Confocal images were captured with a BioRad Radiance 2000 laser-scanning system attached to a Zeiss Axioplan2 microscope. Scanning parameters were optimized for wild type tissues and maintained for scanning the mutant tissues. The immunofluorescence images shown are Z stacks of $4-8$ sections with a scan step of $0.25 \mu \mathrm{m}$. Adobe Photoshop was utilized for assembling all figures. 


\subsection{Results}

\subsubsection{Developmental Expression of 4.1B and Generation of 4.1B null mutants}

Members of the 4.1 protein family are cytoskeletal adaptors that link membrane proteins to the underlying cytoskeleton (Hoover and Bryant, 2000; Peters et al., 1998). 4.1B, a member of this family, is expressed in the nervous system and is highly enriched at the paranodal and juxtaparanodal domains in myelinated axons (Ohara et al., 2000). Based on 4.1B subcellular localization and its binding with Caspr and Caspr2 (Denisenko-Nehrbass et al., 2003; Ohara et al., 2000), it was hypothesized that 4.1B links AGSJs or scaffolds with the axonal cytoskeleton. The primary structure of $4.1 \mathrm{~B}$ contains a 4.1/ezrin/radixin/moesin (FERM) domain, a spectrin/actin binding domain (SAB), a Cterminal domain (CTD) and three unique regions U1-U3 (Fig. 2.1A). We generated antibodies to the U3 unique region of 4.1B (Fig. 2.1A, black bar) and carried out immunostaining of teased sciatic nerve fibers (PNS) and spinal cord sections (CNS) to establish the subcellular localization of 4.1B in the PNS and CNS (Fig. 2.1B, C). As shown in Fig. 2.1B, 4.1B is enriched at the paranodes and JXPs (Ba, red arrows) and overlaps with the paranode-specific protein, Caspr (Fig. 2.1Bb). A merged image (Fig. $2.1 \mathrm{Bc}$ ) clearly shows that $4.1 \mathrm{~B}$ is enriched in the juxtaparanodal region (red arrow) and is not expressed at the nodes (Fig. 2.1Bc, arrow). Similarly, in the CNS, 4.1B is enriched at the paranodes and JXPs, but is not present at the nodes (Fig, 2.1Ca-c). To determine when 4.1B expression begins during postnatal $(\mathrm{P})$ development, we carried out immunoblot analysis of 4.1B from P2 to P24 (Fig. 2.1D). This revealed that two major isoforms of $4.1 \mathrm{~B}(145 \mathrm{kDa}$ and $125 \mathrm{kDa})$ are expressed in the spinal cord throughout early postnatal development. We immunoblotted the lysates against Caspr, which revealed that 
Caspr is also expressed throughout postnatal development, as has been reported previously (Bhat et al., 2001).

$4.1 B$ mouse mutants have been previously generated using conventional methods (Yi et al., 2005). As these presumed 4.1B null mutants did not reveal any developmental or behavioral deficits, concerns were raised whether some 4.1B protein isoforms might still be expressed from the disrupted $4.1 B$ locus. To address this issue, we took advantage of the detailed $4.1 B$ genomic information to generate a floxed allele of $4.1 B$ that would essentially ablate all isoforms of 4.1B upon Cre-mediated recombination (Fig. 2.1E; refer Materials and Methods section). A targeting construct was generated in which exon 6 (which encodes a portion of the FERM domain) was flanked with loxP sites (Fig. 2.1E) and deletion of exon 6 would cause a frameshift resulting in a premature stop codon in exon 7 (Fig. 2.1E, red asterisk). ES cell clones that carried a properly targeted 4.1B floxed allele were identified by Southern analysis (Fig. 2.1F). After germline transmission, mice were genotyped using a combination of primers (Fig. 2.1E), which revealed various genotypes (Fig. 2.1G). Homozygous $4.1 B^{\text {Flox }}$ mice were born with expected Mendelian frequency and did not reveal any obvious behavioral or development deficits (data not shown). After Cre-mediated recombination using the ubiquitous $\beta$ Actin-Cre (Act-Cre), littermate mice with a wild type genotype (+/+) or $\beta$-ActCre $; 4.1 B^{\text {Flox }}$ were analyzed by immunoblotting. As shown in Fig. $2.1 \mathrm{H}$, spinal cord lysates from $\beta$-Act-Cre;4.1 $B^{\text {Flox }}$ show complete loss of 145 and $125 \mathrm{kDa} 4.1 \mathrm{~B}$ protein isoforms, which were present in the wild type lysates, indicating that 4.1B was ablated in $\beta$-Act-Cre; $4.1 B^{\text {Flox }}$ mice. To ensure that 4.1B was also absent in the PNS myelinated axons, we carried out immunostaining against 4.1B, paranodal Caspr and nodal AnkG 
(ANK) in teased sciatic nerves from littermate wild type $(+/+)$ and $\beta$-Act-Cre; $4.1 B^{\text {Flox }}$ mice (Fig. 2.1I, J). As shown in Fig. 2.1J, 4.1B immunoreactivity is completely absent in $\beta$-Act-Cre;4.1B ${ }^{\text {Flox }}$ fibers (Fig. 2.1Ja, red, compare with Ia, Id). Interestingly, the paranodal localization of Caspr in $\beta$-Act-Cre $; 4.1 B^{\text {Flox }}$ sciatic nerve fibers was severely altered, displaying punctate and stretched localization (Fig. 2.1Jb, d, green, compare with Ib, d). The nodal ANK was not affected (Fig. 2.1Jc, d, arrows, compare with Ic, Id). To further confirm that this altered Caspr localization reflected a disorganization of other paranodal proteins, we immunostained $\beta$-Act-Cre; $4.1 B^{\text {Flox }}$ sciatic nerve fibers against Neurofascin (NFAS, which recognizes both NF155 and NF186), Caspr and ANK. The wild type fibers revealed a typical paranodal localization of NF155 (Fig. 2.1Ka, NFAS, green arrowheads) and Caspr (Fig. 2.1 Kb, red arrowheads) at the paranodes and ANK (Fig. 2.1Kc, d, blue, arrow) at the nodes and all in the merged image (Fig. 2.1Kd). In $\beta$ Act-Cre; $4.1 B^{\text {Flox }}$ sciatic nerve fibers both Caspr and NF155 localization at the paranodes was disrupted, and their distribution stretched away from the paranodes (Fig. 2.1La, b, d, asterisks). The ANK localization was normal at the nodes (Fig. 2.1Lc, d, blue, arrow). Together, these results demonstrate that we have generated $4.1 \mathrm{~B}$ null mutants and that loss of $4.1 \mathrm{~B}$ in $\beta$-Act-Cre; $4.1 B^{F l o x}$ mice affects the localization of paranodal proteins in myelinated axons. These results are in contrast to Horresh et al. (2010), which reported that paranodal proteins are not altered in $4.1 B$ mutants (see below). 


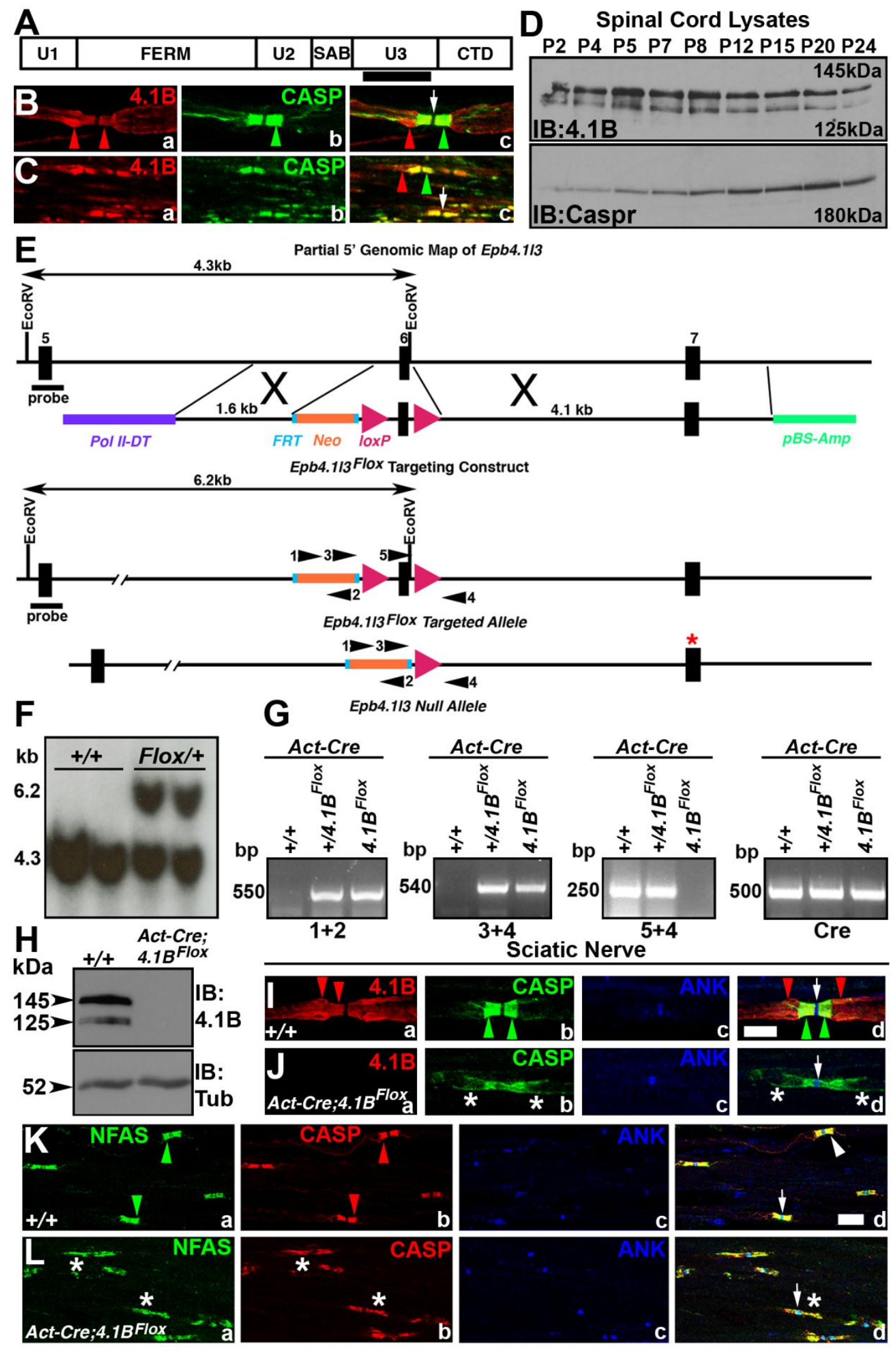


Figure 2.1 Primary Structure and Subcellular Localization of 4.1B and Generation of $4.1 B^{\text {Flox }}$ Mice.

(A) Schematic of the primary structure of mouse 4.1B (deduced from GenBank accession number AF152247) (Parra et al., 2000) shows a Four-point one (4.1) protein, Ezrin, Radixin, Moesin (FERM) domain, a spectrin/actin binding (SAB) domain, a C-Terminal Domain (CTD) and three unique (U1, U2, U3) domains that are specific to each 4.1 protein. The black bar represents a region in the $\mathrm{U} 3$ domain of 4.1B that was used to generate 4.1B antibodies. (B, C) Wild type teased sciatic nerve fibers (B) and spinal cord sections (C) immunostained with anti-4.1B (red) and Caspr (green) showing enrichment of 4.1B at the paranodes and the JXPs (Ba, Ca, red arrowheads) and Caspr at the paranodes (Bb, $\mathbf{C b}$, green arrowheads). Note that 4.1B extends beyond the paranodal area (Bc, Cc, red arrowheads). (D) Immunoblots of wild type spinal cord lysates showing developmental expression of 4.1B (upper panel) and Caspr (lower panel).

(E) A partial genomic map of the 4.1B (Epb4.113) locus showing exons 5-7. A targeting construct with loxP sites flanking exon 6. Cre-mediated recombination of the loxP sites resulted in the deletion of exon 6, which inserted a premature stop codon in exon 7 (red asterisk). (F) Southern blot analysis confirming the targeted $4.1 B^{F l o x}$ allele. (G) PCR amplification of genomic DNA from wild type $(+/+)$, heterozygous Floxed $\left(+/ 4.1 B^{F l o x}\right)$, homozygous Floxed $\left(4.1 B^{F l o x}\right)$, and $\beta$-Act-Cre-expressing (Act-Cre) mice. Primer combinations used are shown in (E). (H) Immunoblot analysis of wild type $(+/+)$ and $\beta$ Act-Cre;4.1B $B^{\text {Flox }}\left(4.1 B^{\text {Act-Flox }}\right.$ ) spinal cord lysates using anti-4.1B (upper panel) and $\alpha$ Tubulin (Tub, lower panel). Note the loss of both isoforms of 4.1B in $4.1 B^{\text {Act-Flox }}$ mutants (right lane). (I) Immunofluorescence of teased sciatic nerve fibers from wild type $(+/+)$ (Ia-d) showing typical localization of 4.1B at the paranodes/JXPs (Ia, Id, red arrows), Caspr at the paranodes (CASP, green, Ib, Id, green arrows), and AnkG at the nodes (Ic, Id, blue, arrow). (J) Immunofluorescence of teased sciatic nerve fibers from $4.1 B^{\text {Act-Flox }}$ mutants (Ja-d) showing loss of 4.1B (red, Ja) with disrupted localization of Caspr in the paranodal region (Jb, green asterisks) but normal AnkG localization at the node (Jc, Jd, arrow). (K) Immunofluorescence of teased sciatic nerve fibers from wild type $(+/+)$ (Kad) showing typical localization of NFAS at the paranodes (green arrowheads) and nodes (Ia, Id, green), Caspr at the paranodes (Kb, Kd, red), and AnkG at the nodes (Kc, Kd, blue, arrow). In Kd, arrowhead points to merge of NFAS and CASP at the paranodes and arrow points to the node. (L) Immunofluorescence of teased sciatic nerve fibers from 4.1B $B^{\text {Act-Flox }}$ mutants (Ja-d) showing disrupted NFAS (Ja, green, asterisks) and Caspr localization at the paranodal region (Jb, red, asterisks). AnkG localization at the node is not affected $(\mathbf{L c}, \mathbf{L d})$. Note that localization of both paranodal proteins NFAS and CASP is disorganized. Scale bars: I-L $10 \mu \mathrm{m}$. 


\subsection{B is Required for the Organization and Stabilization of the Paranodal and Juxtaparanodal Regions in Myelinated Axons}

To determine the consequences of loss of 4.1B on axonal domain organization, we decided to analyze both $4.1 B$ mutants, i.e., $\beta$-Act-Cre; $4.1 B^{\text {Flox }}$ (this study) and $4.1 B^{-/}$ generated previously (Yi et al., 2005). To date, no detailed phenotypic characterization of myelinated axons in any $4.1 B$ mutants has been carried out. We examined the localization of well-characterized paranodal and juxtaparanodal proteins in teased sciatic nerve fibers and spinal cord sections (Figs. $2.2 \& 2.3)$. In wild type $(+/+)$ sciatic nerve fibers, 4.1B is enriched at the paranodes and JXPs and also localizes along the length of the internode (Fig. 2.2Aa, red). Caspr served as a marker for the paranodes (Fig. 2.2Ab, green) and the cytoskeletal adaptor protein AnkG(ANK) (Fig. 2.2Ac, blue) was used as a nodal marker. A merged image of all the markers is shown in Fig. 2.2Ad (arrow points at the node). In

one month old $\beta$-Act-Cre; $4.1 B^{\text {Flox }}$ sciatic nerves, $4.1 \mathrm{~B}$ is absent (Fig. 2.2Ba), while AnkG (Fig. 2.2Bc) localization remains intact at the nodes. The Caspr localization is elongated and disrupted at the paranodes in $\beta$-Act-Cre $; 4.1 B^{\text {Flox }}$ sciatic nerves (Fig. 2.2Bb, d, asterisks). At four months, in $\beta$-Act-Cre;4.1 $B^{\text {Flox }}$ sciatic nerves, 4.1B is absent as expected (Fig. 2.2Ca) and AnkG (Fig. 2.2Cc) remains at the nodes. The Caspr localization at the paranodes in 4 month old $\beta$-Act-Cre; $4.1 B^{\text {Flox }}$ sciatic nerves is broken and occasionally stretches towards the JXPs. Further examination of one month and four month old $\beta$-Act-Cre; $4.1 B^{\text {Flox }}$ sciatic nerves revealed that all juxtaparanodal markers $\mathrm{K}_{\mathrm{V}} 1.1$ (Fig. 2.2Ea, d; 2.2Fa, d), PSD95 (data not shown) and Caspr2 (data not shown) were diffuse, mislocalized and failed to localize properly at the JXPs, compared to wild type fibers (Fig. 2.2Da, d; data not shown). In one month old $\beta$-Act-Cre;4.1 $B^{\text {Flox }}$ sciatic 
nerves, paranodal Caspr (Fig. 2.1L b, d; Fig. 2.2Bb, d; Eb, d; asterisks) and NF155 (Fig. 2.1La, d; asterisks) localization were altered and showed diffuse distribution at the paranodes, often stretching into the juxtaparanodal areas. Nodal markers NF186 (Fig. 2.2Ec, d) and ANK (Fig. 2.1L c, d; Fig. 2.2Bc, d) were not affected. In addition, Caspr (Fig. 2.2Cb, d; Fb, d; Hb, d; asterisks) and NF155 (Fig. 2.2Ha, d; arrowhead) localization at the paranodes in four month old $\beta$-Act-Cre $; 4.1 B^{\text {Flox }}$ sciatic nerves were relatively more compact than at one month, but still contained broken and disrupted areas (Hd, arrowhead). These results indicate that loss of 4.1B affects juxtaparanodal organization and proper localization of axo-glial junctional components at the paranodes (Table 1). Next, we examined localization of paranodal Caspr, juxtaparanodal $\mathrm{K}_{\mathrm{V}} 1.1$ and nodal ANK in the sciatic nerves of 3 month $4.1 B^{-/-}$mice (Yi et al., 2005). As expected, 4.1B is absent at the paranodes and JXPs in $4.1 B^{-/-}$mutant fibers (Fig. 2.2Ja, red). Interestingly, as observed by Horresh et al. (2010), Caspr (Fig. 2.2Jb, d; Lb, d, green) localization at the paranodes was not affected in $4.1 B^{-/}$mutant fibers. $\mathrm{K}_{\mathrm{V}} 1.1$ localization at the JXPs is compromised in $4.1 B^{-/-}$mutants (Fig. 2.2La, d, red; asterisks), as is also seen in $\beta$-Act-Cre; $4.1 B^{\text {Flox }}$ mutants (Fig. 2.2Ea, d; Fa, d, red). These data indicate that in $4.1 B^{-/}$mutants, localization of the juxtaparanodal proteins is compromised, while the paranodal components are not affected (Table 1). 

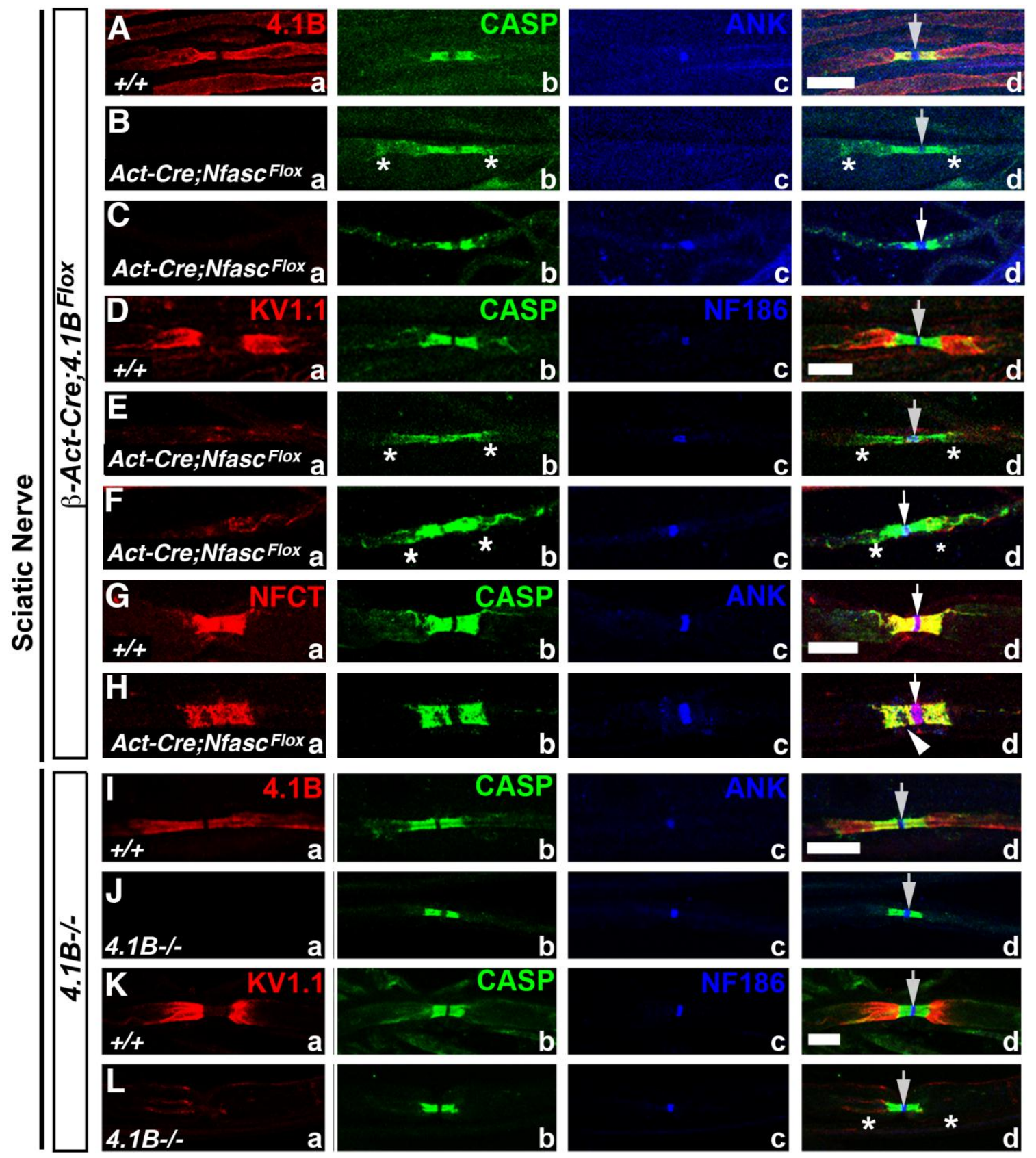
d
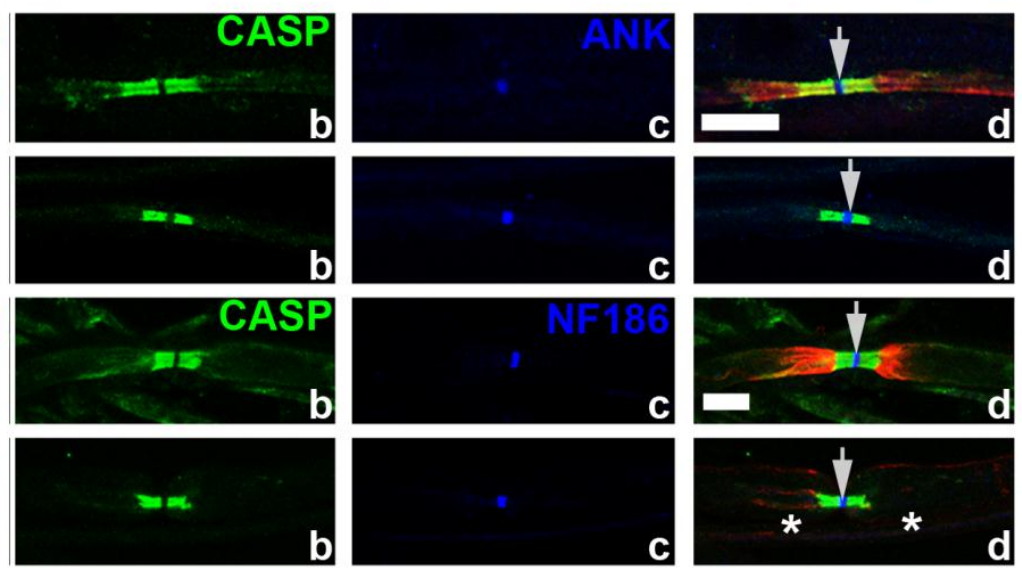

Figure 2.2 Loss of 4.1B Disrupts Paranodal and Juxtaparanodal Organization in the PNS Myelinated Axons.

(A-H) P30 wild type (+/+, Aa-d, Da-d, Ga-d), P30 $\beta$-Act-Cre;4.1B ${ }^{\text {Flox }}$ (Ba-d, Ea-d), and 4month old $\beta$-Act-Cre;4.1B ${ }^{\text {Flox }}$ (Ca-d, Fa-d, Ha-d) sciatic nerve fibers immunostained against 4.1B (Aa, Ba, Ca, red), KV1.1 (Da, Ea, Fa, red), Caspr (Ab-Hb, green), NFct (Ga, Ha), AnkG (Ac-Cc, blue), NF186 (Dc-Hc, blue) and merged images (Ad-Hd). Note that 4.1B is absent from the mutant fibers $(\mathbf{B a}, \mathbf{C a})$ and $\mathrm{K}_{\mathrm{V}} 1.1(\mathbf{E a}, \mathbf{d} ; \mathbf{F a}, \mathbf{d})$ is either undetectable at the JXPs or severely mislocalized. In all $4.1 B$ mutant panels, Caspr (Bb, d; $\mathbf{C b}, \mathbf{d} ; \mathbf{E b}, \mathbf{d} ; \mathbf{F b}, \mathbf{d} ; \mathbf{H b}, \mathbf{d}$, asterisks) and NF155 (NFCT, Ha, d, arrowhead) fail to 
localize properly at the paranodes. Nodal AnkG or NF186 are not affected (Ac,d-Hc,d, arrows).

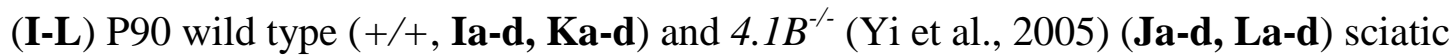
nerve fibers immunostained against 4.1B (Ia, Ja, red), KV1.1 (Ka, La, red), Caspr (IbLb, green), AnkG (Ic, Jc, blue), NF186 (Kc, Lc, blue) and merged images (Id-Ld). 4.1B is absent from the mutant fibers, as expected $(\mathbf{J a}, \mathbf{J d})$ and $\mathrm{K}_{\mathrm{V}} 1.1$ is either undetectable at the JXPs or severely mislocalized (La, Ld, asterisks). However, Caspr localizes properly at the paranodes (Jb, Jd, Lb, Ld, asterisks). Nodal AnkG or NF186 are not affected (Jc,d-Lc,d, arrows). All scale bars: $10 \mu \mathrm{m}$.

Table 2.1 Phenotypic differences between two independently generated $4.1 \mathrm{~B}$ mutant alleles

Phenotype

Sciatic Nerve

Paranodal Proteins

Paranodal AGSJs

JXP proteins

Spinal Cord

Paranodal Proteins

Paranodal AGSJs

JXP proteins
$\beta$-Act-Cre $; 4.1 B^{\text {Flox a }}$

Abnormal localization ${ }^{\mathrm{c}}$

Missing transverse septa

Destabilized AGSJs

Abnormal localization

Abnormal localization $^{\mathrm{e}}$

Missing transverse septa

Destabilized AGSJs

Abnormal localization
$4.1 B^{-/-b}$

Normal localization $^{\mathrm{d}}$

No missing transverse septa

Normal AGSJs

Abnormal localization

Normal localization $^{f}$

Missing transverse septa

Destabilized AGSJs

Abnormal localization

4.1R Localization

Not detected at the paranodes Not detected at the paranodes $4.1 R$ mRNA Levels in DRGs No change

No change

a $4.1 B$ mutants generated in this study. ${ }^{\mathrm{b}} 4.1 \mathrm{~B}$ mutants generated by Yi et al. (2005)

${ }^{c}$ Paranodal proteins Caspr and NF155 show abnormal localization with stretched morphology and gaps. ${ }^{\mathrm{d}}$ Paranodal proteins Caspr and NF155 show normal localization and morphology. ${ }^{\mathrm{e}}$ Paranodal protein Caspr shows normal localization at P30 but at P130, the paranodal areas show gaps and abnormal morphology. ${ }^{\mathrm{f}}$ Paranodal protein Caspr shows relatively normal localization and morphology. 
We also analyzed the localization of paranodal and juxtaparanodal proteins in the spinal cords of $\beta$-Act-Cre $; 4.1 B^{\text {Flox }}$ and $4.1 B^{-/-}$mutants. In wild type $(+/+)$spinal cord sections, 4.1B is enriched at the paranodes and the JXPs (Fig. 2.3Aa, red). In addition, Caspr (Fig. 2.3Ab, Db, green), AnkG (Fig. 2.3Ac, blue), and NF186 (Fig. 2.3Dc, blue) are localized at the paranodes and nodes, respectively (Fig. 2.3Ad, Dd). As in the PNS, $4.1 \mathrm{~B}$ is absent in one month and four month old $\beta$-Act-Cre; $4.1 B^{\text {Flox }}$ spinal cord myelinated axons (Fig. 2.3Ba, Ca, red). However, the paranodal localization of Caspr (Fig. 2.3Bb, d; Eb, d, green) was not disrupted in one month old $\beta$-Act-Cre; $4.1 B^{\text {Flox }}$ spinal cord axons. Interestingly, in four month old $\beta$-Act-Cre; $4.1 B^{\text {Flox }}$ spinal cord myelinated axons, Caspr (Fig. 2.3Cb, d; Fb, d, green) localization was not normal. There were gaps between paranodal Caspr and the nodal proteins AnkG and NF186 (Fig. 2.3Cc, d; Fc, d, arrowheads). Again, the juxtaparanodal $\mathrm{K}_{\mathrm{V}} 1.2$ was mislocalized in both one month and four month old $\beta$-Act-Cre; $4.1 B^{\text {Flox }}$ mutant spinal cord axons (Fig. 2.3Ea, d; Fa, d, red, asterisks). AnkG and NF186 were both maintained at the nodes in all $\beta$-Act-Cre;4.1B ${ }^{\text {Flox }}$ mutants (Fig. 2.3Bc, d; Cc, d; Ec, d; Fc, d, respectively). Analysis of $4.1 B^{-/-}$mutant spinal cord axons also revealed absence of $4.1 \mathrm{~B}$ in $4.1 \mathrm{~B}^{-/-}$mutants (Fig. 2.3Ha, red). Paranodal Caspr localization remained relatively unaffected (Fig. 2.3Hb, d; Jb, d, green). The juxtaparanodal $\mathrm{K}_{\mathrm{V}} 1.1$ was mislocalized (Fig. 2.3Ja, d, red, arrowheads). Nodal AnkG (Fig. 2.3Hc, d, blue) and NF186 (Fig. 2.3Jc, d, blue) were not affected. Together, the immunohistochemical results indicate that 4.1B is critical for proper organization of the juxtaparanodal region in both the PNS and the CNS, and that 4.1B is required for the stabilization of the paranodes in the PNS, and progressively in the CNS (Table 1). 

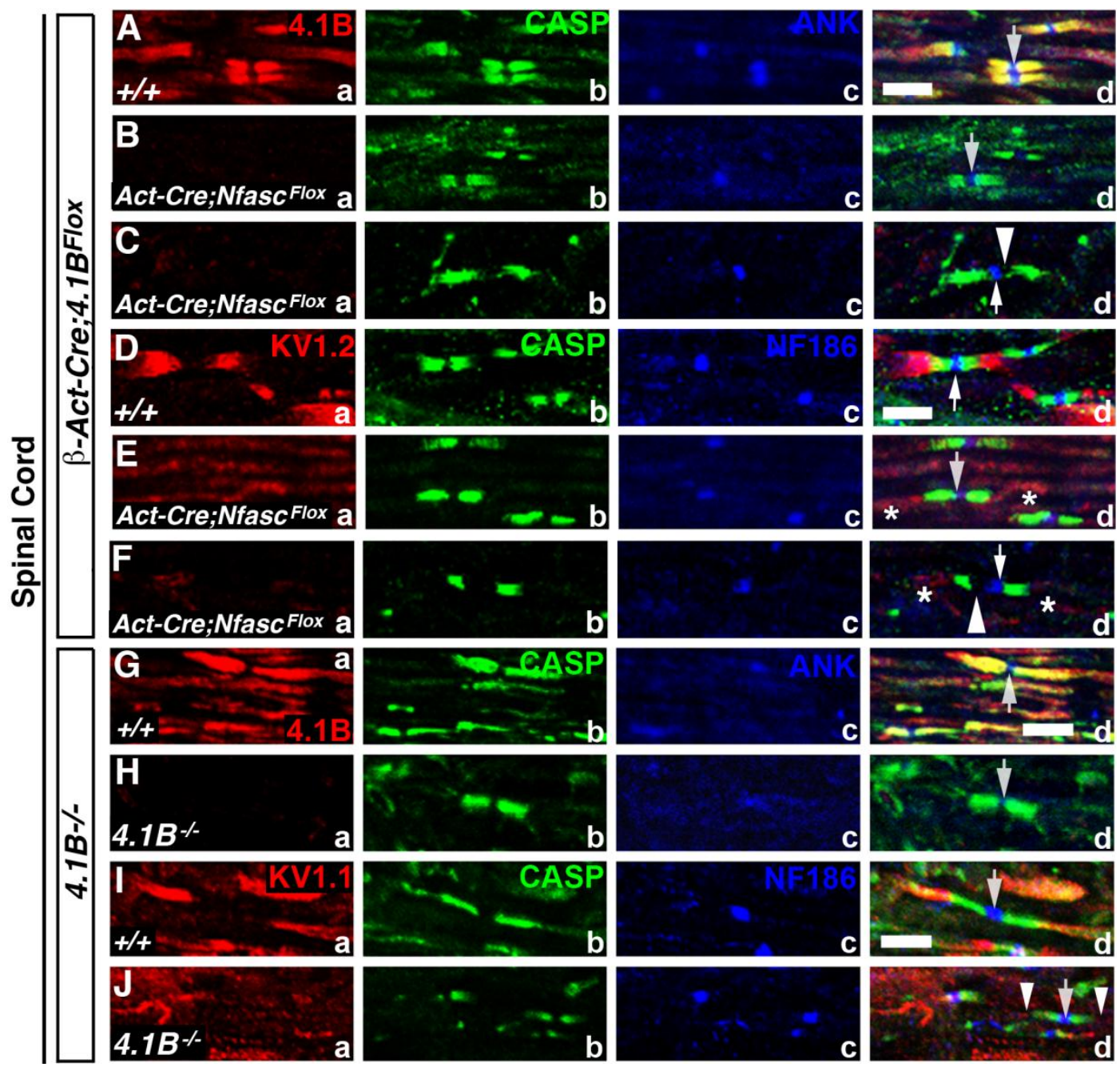

Figure 2.3 Mislocalization of Juxtaparanodal Components and Destabilization of the Paranodes in the CNS Myelinated Axons of 4.1B Mutants.

(A-F) P30 wild type (+/+, Aa-d, Da-d), P30 $\beta$-Act-Cre; $4.1 B^{\text {Flox }}$ (Ba-d, Ea-d), and 4 month old $\beta$-Act-Cre; $4.1 B^{F l o x}$ (Ca-d, Fa-d) spinal cord sections immunostained against 4.1B (Aa, Ba, Ca, red), KV1.2 (Da, Ea, Fa, red), Caspr (Ab-Fb, green), AnkG (Ac, Bc, Cc, blue), NF186 (Dc, Ec, Fc, blue) and merged images (Ad-Fd). Note that 4.1B is absent from the mutant fibers $(\mathbf{B a}, \mathbf{C a})$ and $\mathrm{K}_{\mathrm{V}} 1.1(\mathbf{E a}, \mathbf{d} ; \mathbf{F a}, \mathbf{d})$ is either undetectable at the JXPs or severely mislocalized (Ed, Fd, asterisks). In P30 4.1 $B^{F l o x}$ mutants, Caspr localization in the CNS paranodes is not severely affected. In 4 month old $4.1 B^{\text {Flox }}$ mutants, gaps form between the node and Caspr localization (Cd, Fd, arrowheads). (G-J) P90 wild type (+/+, Ga-d, Ia-d) and $4.1 B^{-/}$(Ha-d, Ja-d) spinal cord sections immunostained against 4.1B (Ga, Ha, red), $\mathrm{K}_{\mathrm{V}} 1.1$ (Ia, Ja, red), Caspr (Gb-Jb, green), AnkG (Gc, Hc, blue), NF186 (Ic, Jc, blue) and merged images (Gd-Jd). Note that 4.1B is absent as expected (Ha) and $\mathrm{K}_{\mathrm{V}} 1.1(\mathbf{J a}, \mathbf{J d})$ is either undetectable at the JXPs or severely mislocalized (Jd, arrowheads). Caspr localization in the CNS paranodes in $4.1 B$ ${ }^{\prime}{ }^{-}$is not severely affected. All scale bars: $5 \mu \mathrm{m}$. 


\subsubsection{Molecular Interactions Between Paranodal Caspr and Complex Formation Between Juxtaparanodal Caspr2, $\mathrm{K}_{\mathrm{v}} 1.2$ and 4.1B}

To further investigate the striking phenotypic differences between the PNS and CNS myelinated fibers, we immunoblotted sciatic nerve and spinal cord lysates from wild type $(+/+), 4.1 B^{-/-}$and $\beta$-Act-Cre $; 4.1 B^{F l o x}$ mutants (Fig. 2.4A, B). Surprisingly, we discovered that the wild type sciatic nerves express close to 8 isoforms of $4.1 \mathrm{~B}$, some with molecular weights exceeding $180 \mathrm{kDa}$, in contrast to the CNS, where only two major isoforms (145 and $125 \mathrm{kDa}$ ) are expressed (compare Fig. 2.4Aa with Fig. 2.4Ba). All of these 4.1B isoforms were absent in both $4.1 B$ mutants (Fig. 2.4Aa, Ba). Immunoblotting against Caspr did not reveal any significant changes in the relative Caspr levels between wild type and $4.1 B$ mutants (Fig. 2.4Ab, Bb). For protein loading control, membranes were immunoblotted against $\alpha$-Tubulin (Tub) (Fig. 2.4Ac, Bc). We also wanted to determine whether disruption of the paranodal axo-glial junctions in Caspr (Bhat et al., 2001) and Cgt (Dupree et al., 1999) mutants affected 4.1B protein levels in the spinal cords. As shown in Fig. 2.4Ca, 4.1B protein levels are relatively unchanged between wild type and the paranodal mutants. Similarly, Caspr levels are not affected in $4.1 B$ and $C g t$ mutants (Fig. $2.4 \mathrm{Cb}$ ). For protein loading control, membranes were immunoblotted against $\alpha$-Tubulin (Fig. 2.4Cc). These data show that sciatic nerves express multiple forms of 4.1B and that loss of 4.1B or Caspr only affects the subcellular localization of Caspr and 4.1B, respectively, and that stable expression of each of these proteins is not dependent on the other paranodal proteins.

Our immunohistochemical data showed that 4.1B is required for the localization of potassium channel $\mathrm{K}_{\mathrm{V}} 1.1$ and Caspr2 to the juxtaparanodal region (Figs. $2.2 \& 2.3$ ). 
Previous studies revealed that a possible mechanism for potassium channel retention at the juxtaparanodal region could be due to molecular interactions between Caspr2 and potassium channels (Horresh et al., 2010; Poliak et al., 2003). We therefore examined whether $4.1 \mathrm{~B}$ is required for Caspr 2 interactions with potassium channels. It is established that 4.1B interacts with Caspr (Denisenko-Nehrbass et al., 2003; GarciaFresco et al., 2006; Ogawa et al., 2006), and Caspr2 (Denisenko-Nehrbass et al., 2003; Horresh et al., 2010). As shown in Fig. 2.4Da, b, immunoprecipitation with anti-Caspr antibodies using spinal cord lysates, co-precipitated 4.1B. Additionally, in the wild type spinal cord lysates, IPs with anti-Caspr2 antibodies co-precipitated potassium channels $\mathrm{K}_{\mathrm{V}} 1.2$ (Fig. 2.4Ea, a'). Caspr2 and $\mathrm{K}_{\mathrm{V}} 1.2$ still formed a molecular complex in the absence of $4.1 \mathrm{~B}$ in $\beta$-Act-Cre; $4.1 B^{\text {Flox }}$ (Fig. 2.4Eb, b') and $4.1 B^{-/-}$(Fig. 2.4Ec, c') spinal cord lysates. These results suggest that $4.1 \mathrm{~B}$ is critical for the maintenance of the juxtaparanodal complex of Caspr2 and potassium channels and not the formation of this molecular complex. Together, our results demonstrate that $4.1 \mathrm{~B}$ is a critical component of the paranodal and juxtaparanodal domains and that expression of multiple isoforms in the PNS versus the CNS may allow for differential function of 4.1B in axonal domain organization. 
Sciatic nerve

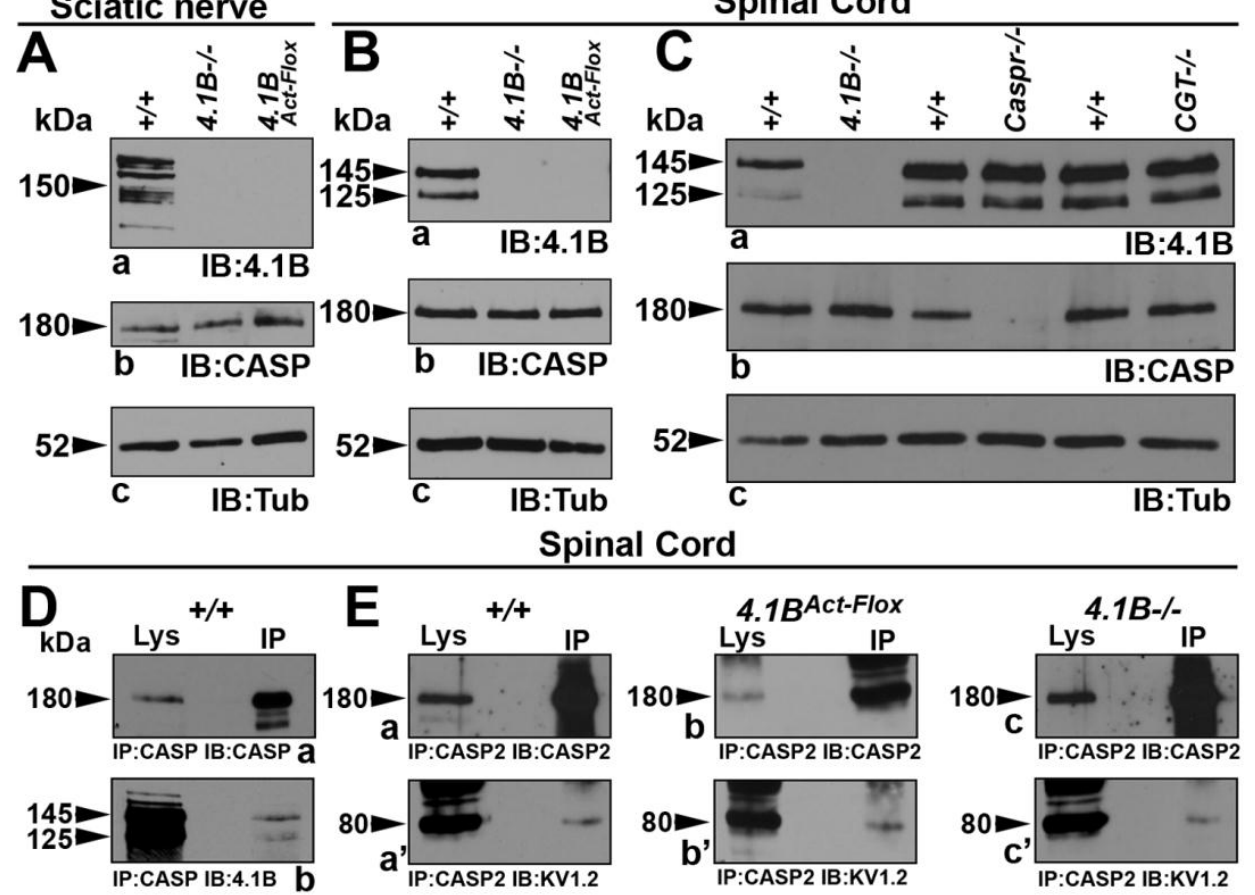

Figure 2.4 Multiple 4.1B Isoforms in Sciatic Nerves and Molecular Interactions between 4.1B, Caspr and Caspr2.

(A) Immunoblot analysis of wild type $(+/+), 4.1 B^{-/-}$and $\beta$-Act-Cre $; 4.1 B^{\text {Flox }}\left(4.1 B^{\text {Act-Flox }}\right)$ sciatic nerve lysates against 4.1B (Aa), Caspr (Ab) and Tubulin (Ac) reveal expression of approximately 8 isoforms of $4.1 \mathrm{~B}$ in the sciatic nerves, which are absent in both $4.1 \mathrm{~B}$ mutants. Caspr levels are unchanged in $4.1 B$ mutants.

(B) Immunoblot analysis of wild type $(+/+), 4.1 B^{-/-}$and $\beta$-Act-Cre; $4.1 B^{\text {Flox }}\left(4.1 B^{\text {Act-Flox }}\right)$ spinal cord lysates against 4.1B (Ba), Caspr $(\mathbf{B b})$ and Tubulin $(\mathbf{B c})$ reveal expression of 2 major isoforms in the CNS, which are absent in both $4.1 B$ mutants. Note that Caspr levels are not affected in $4.1 B$ mutants.

(C) Immunoblot analysis of wild type $(+/+), 4.1 B^{-/}$, Caspr $^{-/}$, and $\mathrm{Cgt}^{-/}$spinal cord lysates against 4.1B (Ca), Caspr $(\mathbf{C b})$ and Tubulin $(\mathbf{C c})$ showed that the levels of 4.1B and Caspr are not affected in Caspr and $4.1 B$ mutants, respectively. Cgt mutants also did not show any changes in $4.1 \mathrm{~B}$ expression.

(D) Immunoprecipitation (IP) analysis using anti-Caspr antibodies (Da) with wild type $(+/+)$ spinal cord lysates co-IPs 4.1B (Db).

(E) IP analysis using anti-Caspr2 antibodies with wild type $(+/+$, Ea, a'), $\beta$-ActCre $; 4.1 B^{\text {Flox }}\left(4.1 B^{\text {Act-Flox }}, \mathbf{E b}, \mathbf{b}^{\prime}\right)$ and $4.1 B^{-/}$(Ec, $\left.\mathbf{c}^{\prime}\right)$ spinal cord lysates co-IPs $\mathrm{K}_{\mathrm{V}} 1.2$ in all the genotypes suggesting that Caspr2 interactions with $\mathrm{K}_{\mathrm{V}} 1.2$ are independent of $4.1 \mathrm{~B}$. 


\subsection{B is Critical for the Stability of the Paranodal Axo-Glial Septate Junctions}

Our immunofluorescence studies revealed striking juxtaparanodal disorganization in both the PNS and the CNS in $\beta$-Act-Cre; $4.1 B^{\text {Flox }}$ and $4.1 B^{-/-}$mutants (Figs. 2.2 and 2.3). In addition, we found severe paranodal destabilization in the PNS, and progressive paranodal disruption in the CNS, of $\beta$-Act-Cre;4.1B ${ }^{\text {Flox }}$ mice (Fig. 2.3). To further investigate the role of 4.1B in axonal domain organization, we used electron microscopy to specifically look at the ultrastructural architecture of the paranodal region in wild type, $\beta$-Act-Cre $; 4.1 B^{F l o x}$ and $4.1 B^{-/}$mutant sciatic nerve and spinal cord myelinated axons (Figs. 2.5 and 2.6). A low magnification electron micrograph of P30 littermate wild type $\left(+/+\right.$, Fig. 2.5A) and $\beta$-Act-Cre;4.1B $B^{\text {Flox }}$ mutant (Fig. 2.5B) sciatic nerve myelinated axons, at the level of the nodal/paranodal region, shows a typical domain distinction between the nodal and the paranodal region. At this magnification, the wild type and $\beta$ Act-Cre; $4.1 B^{\text {Flox }}$ mutant fibers look essentially similar with respect to the presence of individual domains. At a higher magnification, the paranodal AGSJs show characteristic electron-dense septa between the myelin loops and the axolemma in wild type fibers (Fig. $2.5 \mathrm{C}$, arrowheads). In one month old $\beta$-Act-Cre $; 4.1 B^{F l o x}$ mutant fibers, a few electrondense septa can be found (Fig. 2.5D, arrowhead), while most septa appear diffuse and illdefined (Fig. 2.5D, asterisks), suggesting that septa may be undergoing destabilization. In three month old $\beta$-Act-Cre $; 4.1 B^{\text {Flox }}$ mutant fibers, the electron density of the septa is diffuse and fuzzy (Fig. 2.5E, F, asterisks). In comparison, ultrastructural analysis of previously generated $4.1 B^{-/}$mutants revealed no clear differences in the ultrastructural morphology of the paranodal axo-glial septa at three months (Fig. $2.5 \mathrm{H}$, compare with Fig. 2.5G, arrowheads). However, at 6 months and one year time points, septa were 
missing in the sciatic nerve paranodal regions (data not shown) in $4.1 B^{-/-}$mutants. Quantification of the percentage of paranodes with a normal array of septa in the sciatic nerve of each genotype at three months revealed that in the wild type mice $90 \%$ of paranodes contain a full complement of septa. In sciatic nerve fibers of three month old $\beta$-Act-Cre $; 4.1 B^{\text {Flox }}$ mice, only $60 \%$ of the paranodes counted retained a full complement of septa, while $75 \%$ of paranodes in three month old $4.1 \mathrm{~B}^{-/-}$sciatic nerves contained a normal set of septa. In $\beta$-Act-Cre $; 4.1 B^{\text {Flox }}$ mice paranodes often displayed severe morphological defects, which occluded visualization of missing septa suggesting that paranodal disorganization was more severe than were included in the quantification. Together, these results are consistent with our immunostaining results (Fig. 2.2), which showed disrupted localization of the paranodal proteins in $\beta$-Act-Cre $; 4.1 B^{\text {Flox }}$ mutant sciatic nerve fibers. 


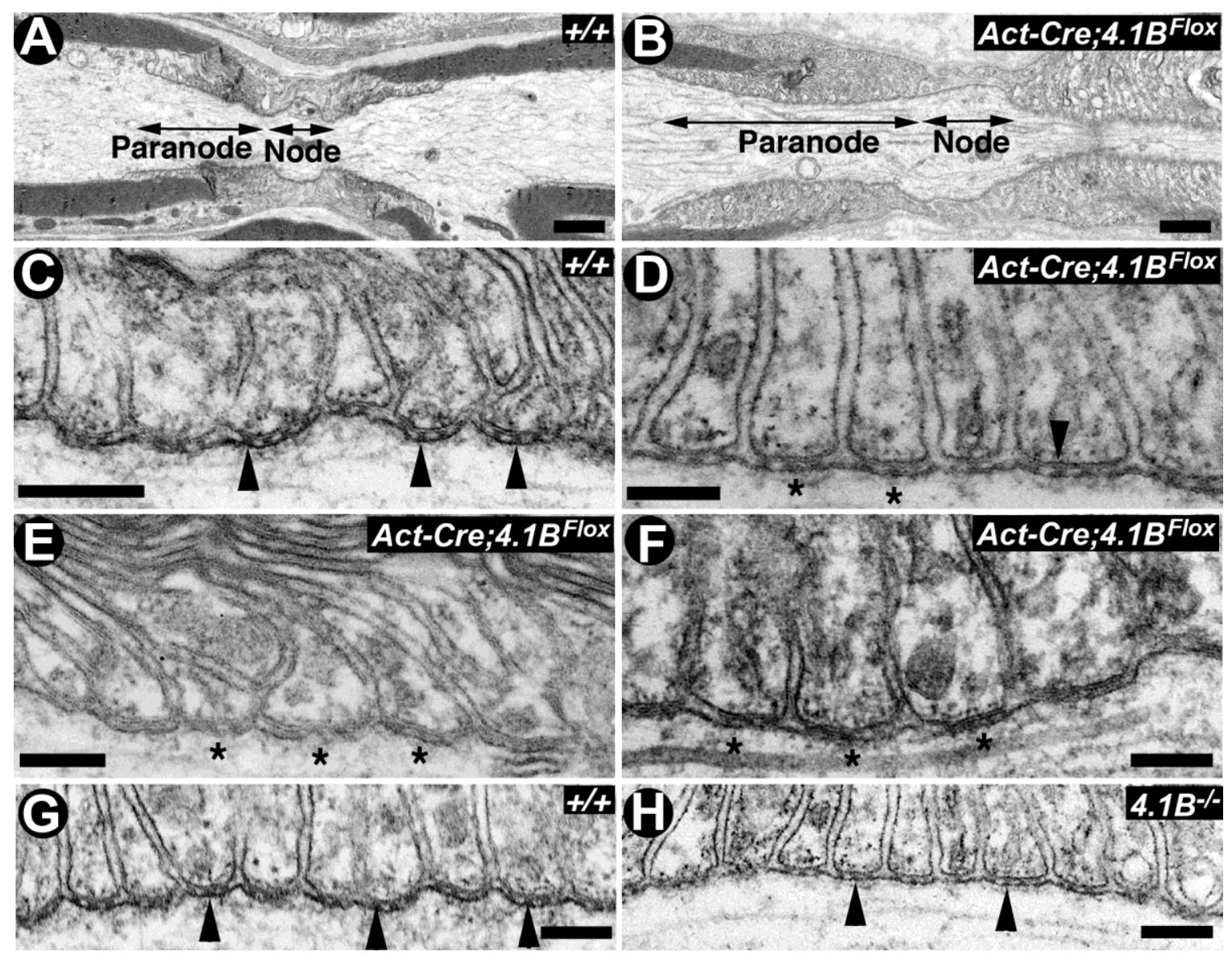

Figure 2.5 4.1B is Required for the Stability of Paranodal Axo-glial Septate Junctions in Sciatic Nerve Myelinated Axons.

(A-D) Electron micrographs of longitudinal sections through the nodal/paranodal regions of sciatic nerves from $\mathrm{P} 30$ wild type $(\mathbf{A}, \mathbf{C},+/+)$ and $\beta$-Act-Cre $; 4.1 B^{\text {Flox }}(\mathbf{B}, \mathbf{D})$. At low magnification, the paranodal myelin loops in the $\beta$-Act-Cre $; 4.1 B^{\text {Flox }}$ mutant fibers are properly arrayed and show normal profiles as in the wild type. At a higher magnification, the wild type $(+/+)$ paranodal loops are tightly apposed and indent the axon, with the characteristic transverse septa ( $\mathbf{C}$, black arrowheads). In $\beta$-Act-Cre $; 4.1 B^{\text {Flox }}$ mutant fibers, the paranodal septa display a fuzzy, ill-defined appearance (D, asterisks).

$(\mathbf{E}, \mathbf{F})$ Electron micrographs of paranodal regions of P90 $\beta$-Act-Cre $; 4.1 B^{\text {Flox }}$ sciatic nerves. Septa are less electron-dense than wild type septa, a sign of destabilization (asterisks).

$(\mathbf{G}, \mathbf{H})$ Electron micrographs of longitudinal sections through the paranodal regions of sciatic nerves from P90 wild type $(\mathbf{J},+/+)$ and $4.1 B^{-/-}$generated by Yi et al. (2005) (K). The paranodal septa in $4.1 \mathrm{~B}^{-/}$mutants display similar morphology and density as is seen in the wild type (+/+) axons. Scale bars: A, B, $1 \mu \mathrm{m} ; \mathbf{C}-\mathbf{H}, 0.2 \mu \mathrm{m}$. 
Next, we analyzed the spinal cord myelinated axons in one month and three month old wild type and $\beta$-Act-Cre;4.1B ${ }^{\text {Flox }}$ mutants. As shown in Fig. 2.6A, wild type spinal cord myelinated axons display a uniform arrangement of septa between the myelin loops and the axolemma (Fig. 2.6A, arrowheads). The fibers from one month old $\beta$-ActCre $; 4.1 B^{\text {Flox }}$ mutant spinal cords revealed the presence of axo-glial septa in some regions (Fig. 2.6B, arrowhead) and complete loss of septa in other regions at the same paranodes (Fig. 2.6B, C, D, asterisks). In the areas where septa are missing, the axolemma showed clear detachments from the myelin loops and loss of electron densities (Fig. 2.6B-D, white arrows). At three months, some electron-densities are still found in $\beta$-Act-Cre $; 4.1 B^{\text {Flox }}$ mutant spinal cords (Fig. 2.6F, arrowhead). However, many paranodal loops were detached from the axonal membrane (Fig. 2.6F, G, arrows). In addition, larger areas were found with missing septa (Fig. 2.6H, white arrow), and detached axolemma (Fig. 2.6G,H, asterisks). Detachment of paranodal loops abutting the node agrees with immunohistochemical data revealing gaps between the nodal proteins and Caspr in 4 month old $\beta$-Act-Cre $; 4.1 B^{\text {Flox }}$ mutant spinal cords (Fig. 2.3Cd, Fd, arrowheads). The $\beta$ Act-Cre $; 4.1 B^{\text {Flox }}$ mutant fibers also revealed accumulation of smooth endoplasmic reticulum (SER) at the nodal/paranodal regions (Fig. 2.6E), suggesting a disruption of the local axonal cytoskeleton, as has been previously observed in Caspr, Cgt (Einheber et al., 2006; Garcia-Fresco et al., 2006) and $N f a s c^{N F 155}$ (Pillai et al., 2009) mutants. These data suggest that $4.1 \mathrm{~B}$ is not required for the initial organization of the paranodal axo-glial septa, but for their stabilization.

To determine the paranodal defects in the spinal cord myelinated axons in $4.1 \mathrm{~B}^{-/}$ mutants, we analyzed them at P90, 6 months and one year time points. As shown in Fig. 
2.6I, P90 wild type spinal cord myelinated axons showed proper densities and arrangement of the septa (arrowheads). In contrast, P90 $4.1 B^{-/-}$mutants showed myelinated axons with normal septa (Fig. 2.6J, arrowheads) and regions with abnormal or missing septa (Fig. 2.6J, asterisks, white arrow). By 6 months, $4.1 B^{-/-}$mutant myelinated axons revealed a more prominent loss of septa with increased spacing between the paranodal lateral loops and the axolemma indicating a progressive deterioration of the paranode with age (Fig. 2.6K, asterisks). In these fibers, destabilization of septa becomes obvious as the densities of these fibers looked diffuse (Fig. 2.6K, arrowheads). Analysis of one year old $4.1 B^{-/}$mutants spinal cord myelinated axons revealed further destabilization of the paranodal regions, with essentially all septa lost and the axolemma detaching from the myelin loops (Fig. 2.6L, M, asterisks). The one year old $4.1 B^{--}$mutant spinal cord myelinated axons also revealed accumulation of organelles, including mitochondria and SER at the nodal/paranodal regions suggesting a disruption of the axonal cytoskeleton, as observed in the sciatic nerves (Fig. 2.6N, arrowheads, data not shown). To directly compare the phenotypes in the two $4.1 B$ null mice, the number of paranodes with a complete set of septa at three months were quantified. In wild type spinal cords, $94 \%$ of paranodes contain a full set of septa. In $\beta$-Act-Cre $; 4.1 B^{\text {Flox }}$ spinal cords, only $36 \%$ of the paranodes retain a complete set of septa, while $50 \%$ of the paranodes in $4.1 \mathrm{~B}^{-/-}$spinal cords maintain a complete set of septa. Interestingly, in the spinal cord of $\beta$-Act-Cre; $4.1 B^{\text {Flox }}$ mice, loss of septa resulted in destabilization of paranodal loops, especially in the region abutting the node (Fig. 2.6F). Quantification revealed that $72 \%$ of paranodes in wild type spinal cords contain a complete set of loops contacting the paranodal axolemma. In the $\beta$-Act-Cre $; 4.1 B^{\text {Flox }}$ spinal cords, only $33 \%$ of 
paranodes maintained loops in contact with the axolemma. In comparison, in $4.1 B^{-/}$ spinal cords, $71 \%$ of paranodes retained a complete set of loops contacting the axolemma. These data suggest that loss of $4.1 \mathrm{~B}$ in $\beta$-Act-Cre; $4.1 \mathrm{~B}^{\text {Flox }}$ spinal cords results in destabilization of the septa and lifting of the paranodal loops from axolemma.

Together, the ultrastructural analyses in $\beta$-Act-Cre $; 4.1 B^{F l o x}$ and $4.1 B^{-/-}$mutants demonstrate that 4.1B is critical for the stabilization of paranodal AGSJs, and that the long-term stability of the AGSJs is dependent on 4.1B, as it provides a scaffolding link with the paranodal axonal cytoskeleton (Table 1). 


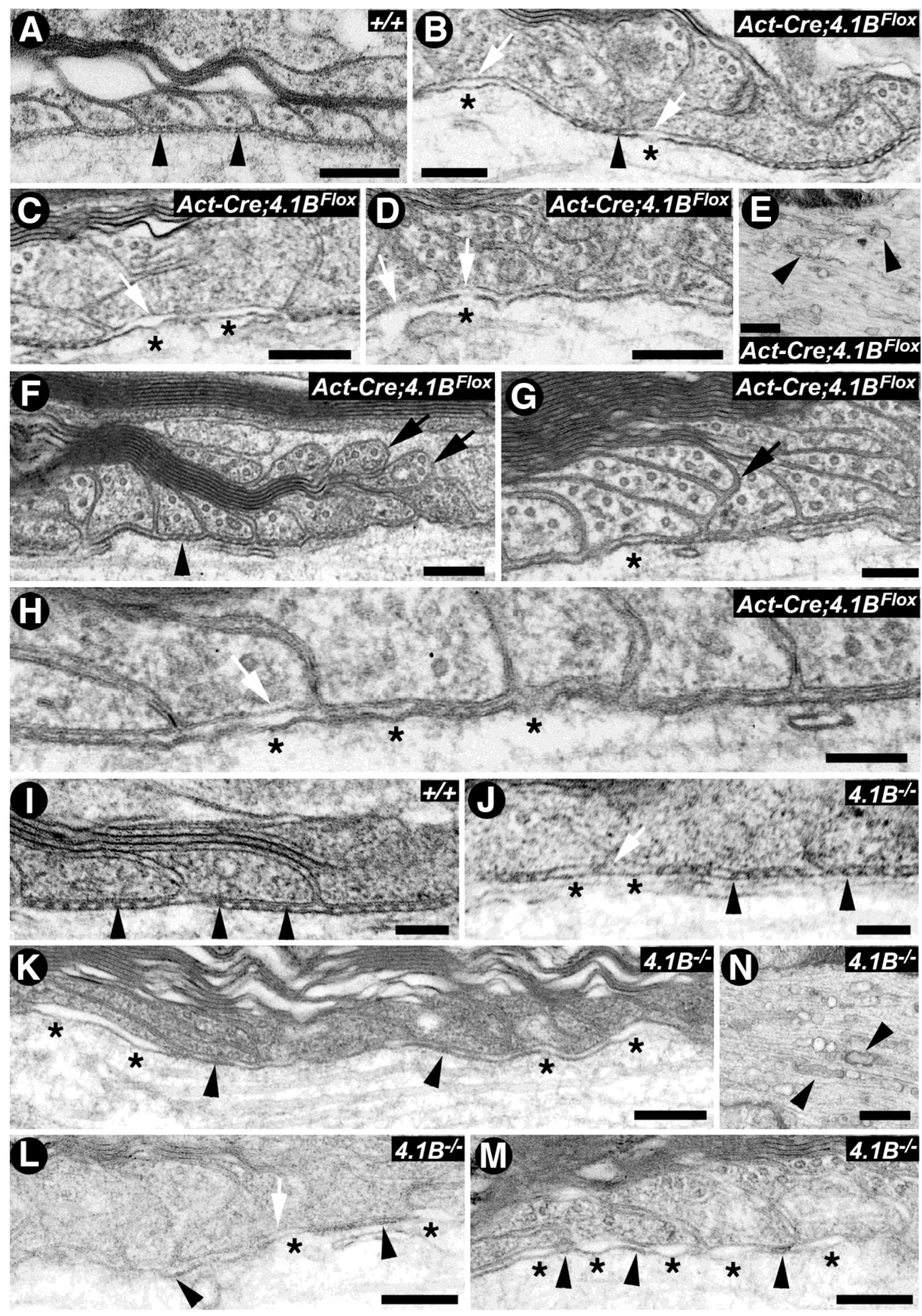


Figure 2.6 4.1B is Required for the Stability of Paranodal Axo-glial Septate Junctions in the Spinal Cord Myelinated Axons.

(A-D) Electron micrographs of the paranodal regions of spinal cord myelinated fibers from P30 wild type $(+/+)$ shows paranodal loops tightly apposed to and indent the axon, with the characteristic septa (A, black arrowheads). In $\beta$-Act-Cre $; 4.1 B^{\text {Flox }}$ mutant spinal cords, the paranodal region displays regions with normal septa (B, arrowheads) and regions where septa are essentially absent $(\mathbf{B}, \mathbf{C}, \mathbf{D}$, asterisks). White arrows point to detached axolemma from the paranodal loops $(\mathbf{B}, \mathbf{C}, \mathbf{D})$ or breaks in the axolemma $(\mathbf{D})$.

(E) $\beta$-Act-Cre; $4.1 B^{\text {Flox }}$ mutant myelinated axons often display accumulation of SER and mitochondria at the nodal/paranodal region, as has been previously observed in Caspr ${ }^{-/}$ mutants.

(F-H) Electron micrographs of the paranodal regions of spinal cord myelinated fibers from P90 $\beta$-Act-Cre; $4.1 B^{\text {Flox }}$ mutants show lifting off of paranodal loops (F, G, arrows), stretches of missing septa $(\mathbf{G}, \mathbf{H})$, and detachment of the axolemma ( $\mathbf{G}, \mathbf{H}$, asterisks), while some septa also remain at this time point (F, arrowhead).

(I-M) Electron micrographs of the paranodal regions of spinal cord myelinated fibers from P90 wild type $(\mathbf{I},+/+)$, P90 4.1B ${ }^{-/}$mutants $(\mathbf{J})$, P180 4.1B $B^{-/}$mutants $(\mathbf{K})$ and P365 $4.1 B^{-/}$mutants $(\mathbf{L}, \mathbf{M})$. The spinal cords of P90 $4.1 B^{-/}$mutants show paranodal regions with normal septa (arrowheads) and regions with abnormal or missing septa (J, K, asterisks). In P365 4.1 $B^{-/}$spinal cords, majority of the paranodes did not display septa with normal morphology, but displayed diffuse electron densities (L, arrowheads). Large regions of the paranodes had essentially lost all the septa (M, asterisks) and displayed thinner axolemma.

(N) $4.1 B^{-/}$myelinated axons from one year old mutants also displayed accumulation of SER and mitochondria at the nodal/paranodal regions (arrowheads), as observed in P30 $\beta$ Act-Cre; $4.1 B^{\text {Flox }}$ mutants (refer panel E). Scale bars: A-D, $0.2 \mu \mathrm{m} ; \mathbf{E}, \mathbf{N}, 0.5 \mu \mathrm{m} ; \mathbf{F}-\mathbf{H}$, $0.1 \mu \mathrm{m} ; \mathbf{I}-\mathbf{M}, 0.2 \mu \mathrm{m}$. 


\subsection{R is Not Expressed at the PNS Paranodes or Enriched in 4.1B ${ }^{-/-}$Mutants}

One of the underlying reasons suggested to explain a lack of phenotype at the PNS paranodes in $4.1 B^{-/}$mutants was that $4.1 \mathrm{R}$ was expressed at the mutant paranodes and not at the wild type paranodes (Horresh et al., 2010). We wanted to determine whether $\beta$-Act-Cre; $4.1 B^{\text {Flox }}$ mutant sciatic nerves also had increased $4.1 \mathrm{R}$ expression at the paranodes, and as controls we used $4.1 B^{-/}$mutants. Immunostaining of wild type $(+/+), \beta$-Act-Cre $; 4.1 B^{\text {Flox }}$ and $4.1 B^{-/-}$sciatic nerve fibers against $4.1 \mathrm{R}$ did not reveal expression of 4.1R at paranodes in any of the genotypes analyzed (Fig. 2.7Aa, c-Fa, c, red, arrowheads). Caspr localization was normal in the wild type and $4.1 B^{-/-}$mutant fibers and disrupted in $\beta$-Act-Cre $; 4.1 B^{\text {Flox }}$ mutant fibers (data not shown). These data show that 4.1R is not expressed at the paranodes and thus cannot possibly compensate for loss of 4.1B at the paranodes. To test the specificity of our 4.1R antibody, we carried out immunoblot analysis of sciatic nerves from wild type and $4.1 R^{-/}$mice and confirmed that 4.1R was missing in $4.1 R^{-/}$sciatic nerves (Fig. 2.7G) (Shi et al., 1999). We also carried out immunoblot analysis of sciatic nerves and spinal cords from littermate wild type and $\beta$-Act-Cre $; 4.1 B^{\text {Flox }}$ and $4.1 B^{-/-}$mutants. As shown in Fig. $2.7 \mathrm{H}$ and 2.7I, no significant differences, and especially no increases, were observed in 4.1R levels between wild type and both $4.1 B$ mutants. Immunoblotting against $\alpha$-Tubulin (Tub) was used as a loading control. To confirm these results, we also completed RT-PCR with RNA extracted from dorsal root ganglia of littermate wild type and $\beta$-Act-Cre $; 4.1 B^{\text {Flox }}$ and $4.1 B^{-/-}$mutants (Fig. 2.7J). Primers targeted to amplify DNA from exons 5 through 8 were used to show deletion of exon 6 in the $\beta$-Act-Cre $; 4.1 B^{\text {Flox }}$ mice (Fig. 2.7J). The genotype of $4.1 B^{-/-}$ mutants were confirmed using primers designed to exons 2 through 3 , resulting in loss of 
the band in this mutant only (data not shown). Importantly, 4.1R levels were uniform in all genotypes (Fig. 2.7J). Actin was used as a house-keeping gene control. Together the immunostaining, immunoblot, and RT-PCR analyses in two independent $4.1 B$ mutants show that $4.1 \mathrm{R}$ is not expressed at the paranodes in the wild type fibers and that $4.1 \mathrm{R}$ does not get enriched at $4.1 B$ mutant paranodes (Table 1). 

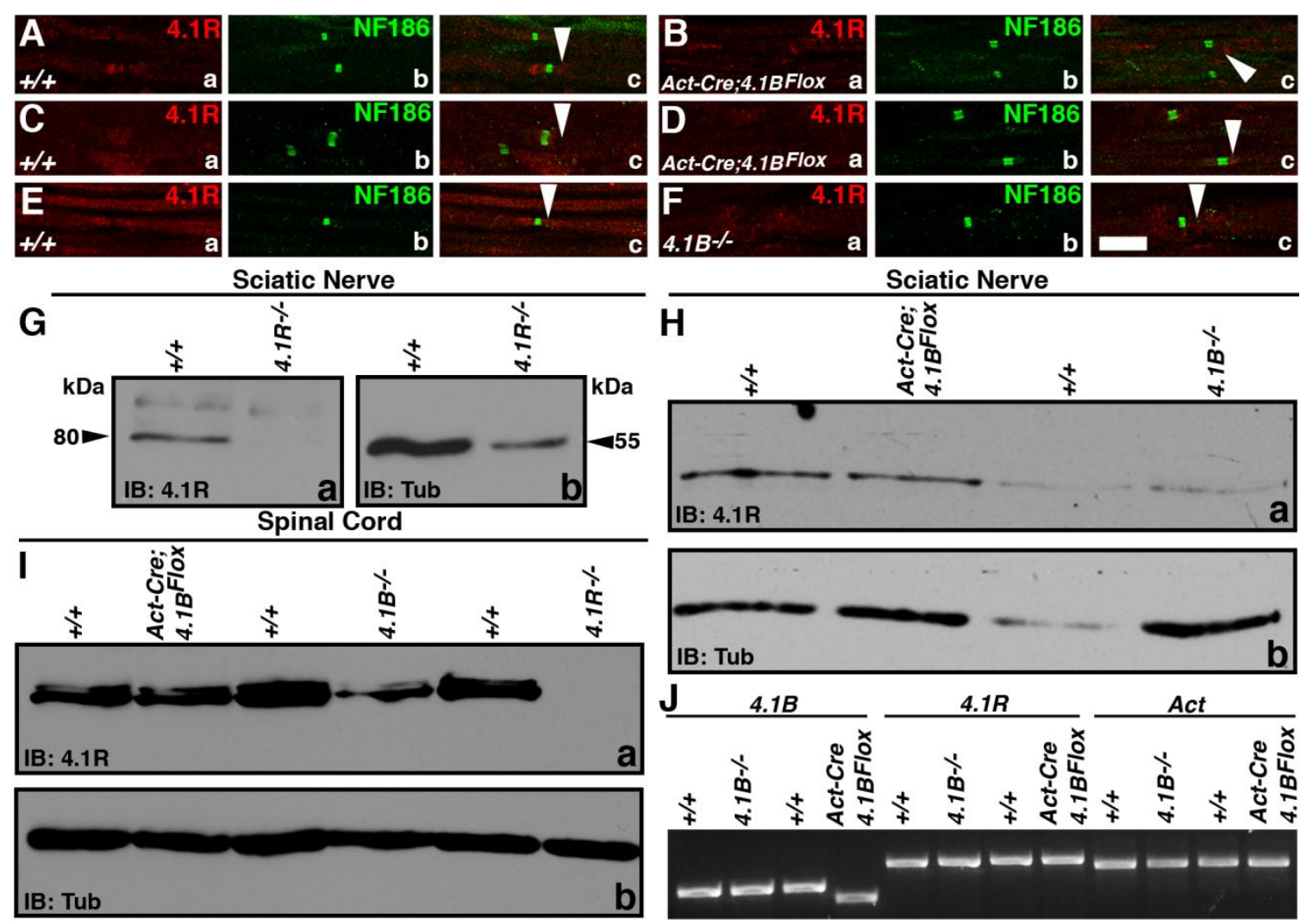

Sciatic Nerve

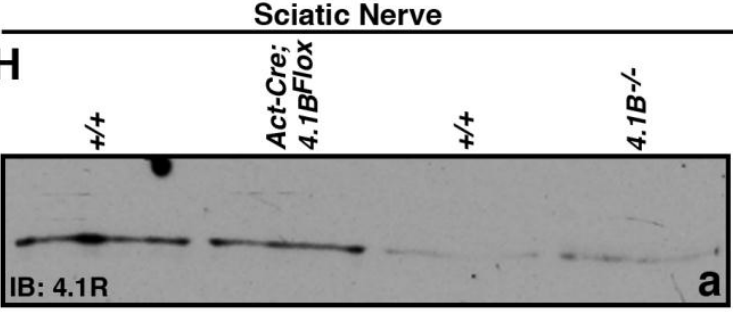

Figure 2.7 4.1R is not Enriched at Sciatic Nerve Paranodes in 4.1B Mutants.

(A-F) P30 wild type (+/+, Aa-d; Ca-d; Ea-d), $\beta$-Act-Cre;4.1B $B^{\text {Flox }}\left(\right.$ Act-Cre;4.1B ${ }^{\text {Flox }}$, Bad; Da-d), and $4.1 B^{-/-}$(Fa-d) sciatic nerves immunostained against 4.1R (Aa, c-Fa, c, red, arrowheads) and NF186 (Ab-Fb, green) and in merged images (Ac-Fc). Note that 4.1R is not expressed or enriched at mutant paranodes in any of the genotypes.

(G) Immunoblot analysis of wild type $(+/+)$ and $4.1 R^{-/-}$sciatic nerve lysates against $4.1 \mathrm{R}$ (Ga) and $\alpha$-Tubulin (Tub, Gb) reveal the specificity of the $4.1 \mathrm{R}$ antibody with the $80 \mathrm{kDa}$ 4.1R present in wild type lysate, but not $4.1 R^{-/-}$lysate $(\mathbf{G a})$.

(H) Immunoblot analysis of wild type $(+/+), \beta$-Act-Cre $; 4.1 B^{\text {Flox }}\left(4.1 B^{\text {Act-Flox }}\right)$, and $4.1 B^{-/-}$ sciatic nerve lysates against 4.1R (Da) and $\alpha$-Tubulin (Tub, Db) reveal no change in 4.1R levels in either of the $4.1 B$ mutants, compared to wild type.

(I) Immunoblot analysis of wild type $(+/+), \beta$-Act-Cre; $4.1 B^{\text {Flox }}\left(4.1 B^{\text {Act-Flox }}\right)$, and $4.1 B^{-/-}$ spinal cord lysates against 4.1R (Ia) and $\alpha$-Tubulin (Tub, Ib) reveal no change in $4.1 \mathrm{R}$ expression in both $4.1 B$ mutants (asterisks), compared to wild type.

(J) RT-PCR analyses of wild type (+/+), $\beta$-Act-Cre;4.1B ${ }^{\text {Flox }}$ (Act-Cre; $4.1 B^{\text {Flox }}$ ), and $4.1 B$ - dorsal root ganglia utilizing primers specific to $4.1 B, 4.1 R$ and Actin (Act) reveal no change in the levels of $4.1 R$ mRNA in either of the $4.1 B$ mutants, compared to wild type. Scale bars: $10 \mu \mathrm{m}$. 


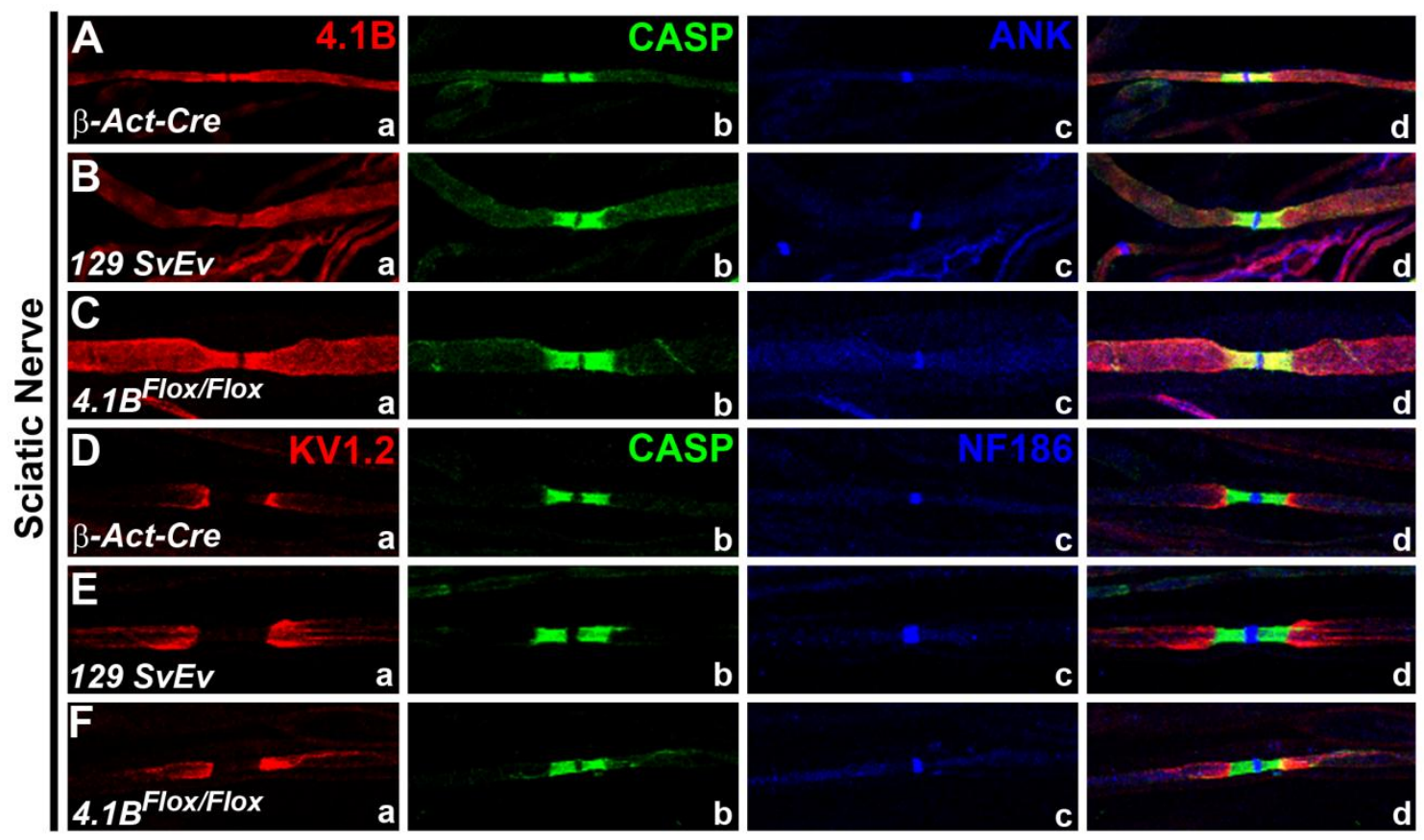

Figure 2.8 Paranodal and juxtaparanodal proteins are not disrupted in the $\beta$-ActinCre $;$ 4.1B ${ }^{\text {Flox }}$ background strains.

(A) $\beta$-Actin-Cre sciatic nerves immunostained against 4.1B (Aa, red), Caspr (Ab, green), nodal AnkG (Ac, blue), and merged image (Ad). (B) $129 S v E v$ sciatic nerves immunostained against 4.1B (Ba, red), Caspr (Bb, green), AnkG (Bc, blue) and merged image (Bd). (C) $4.1 B^{\text {Flox/Flox }}$ sciatic nerves immunostained against 4.1B (Ca, red), Caspr $(\mathbf{C b}$, green), AnkG (Cc, blue) and merged image $(\mathbf{C d})$. Note that $4.1 \mathrm{~B}$ is enriched at the paranodes and JXPs and uniformly at lower levels throughout the axon. Caspr is also localized properly to the paranode and AnkG to the node in all fibers. (D) $\beta$-Actin-Cre sciatic nerves immunostained against $\mathrm{K}_{\mathrm{V}} 1.2$ (Da, red), Caspr (Db, green), nodal NF186 (Dc, blue), and merged image (Dd). (E) $129 S v E v$ sciatic nerves immunostained against $\mathrm{K}_{\mathrm{V}} 1.2$ (Ea, red), Caspr (Eb, green), NF186 (Ec, blue) and merged image (Ed). (F) $4.1 B^{\text {Flox/Flox }}$ sciatic nerves immunostained against $\mathrm{K}_{\mathrm{V}} 1.2$ (Fa, red), Caspr (Fb, green), NF186 (Fc, blue) and merged image $(\mathbf{F d})$. Note that $\mathrm{K}_{\mathrm{V}} 1.2$ is localized properly at the JXPs, Caspr at the paranodes, and NF186 at the nodes in all fibers. 


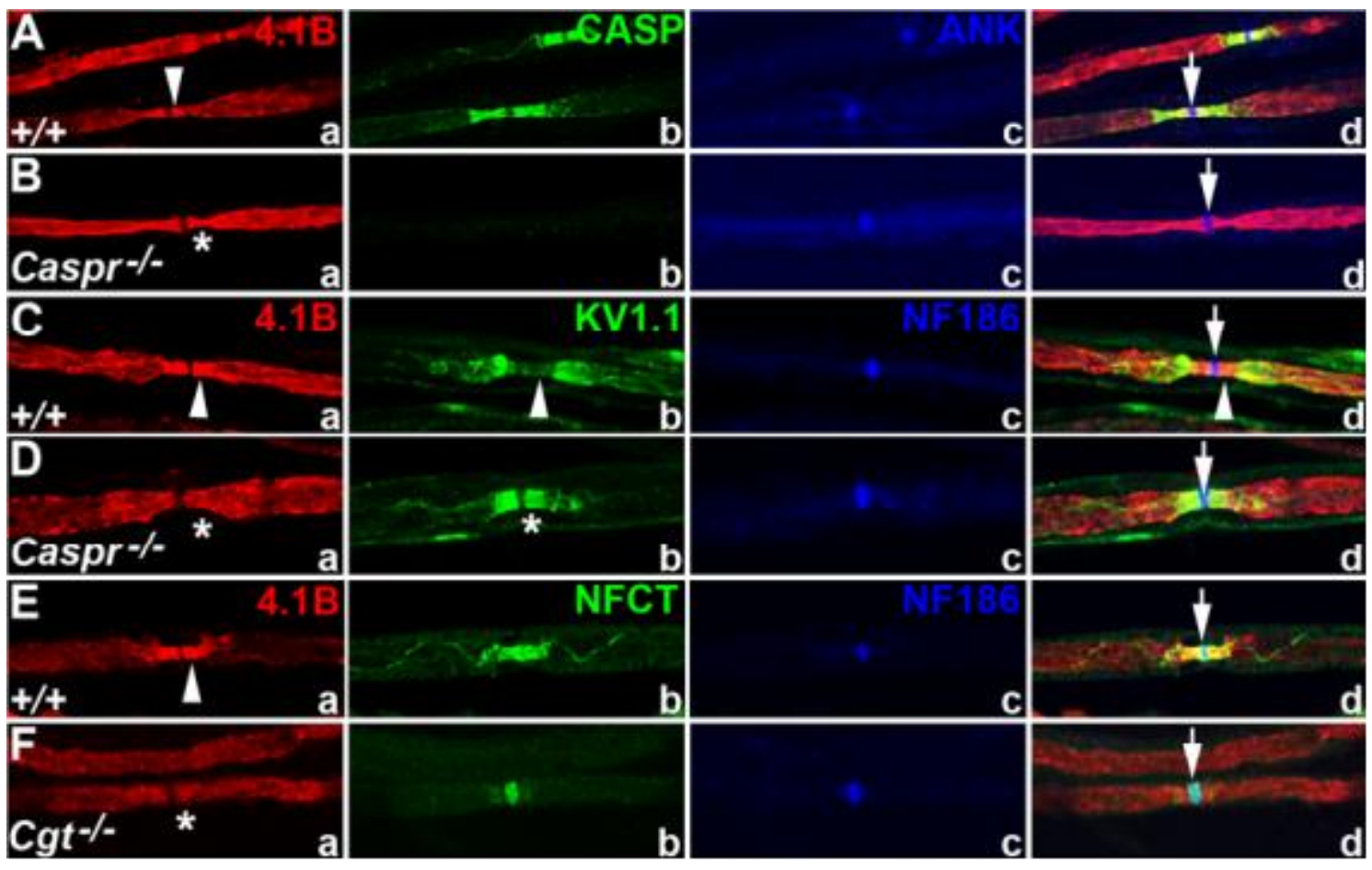

Figure 2.9 Enrichment of 4.1B at the paranodal and juxtaparanodal domains is severely affected in Caspr and Cgt Mutants.

(A) Wild-type sciatic nerves immunostained against 4.1B (Aa, red), Caspr (Ab, green), nodal AnkG (Ac, blue), and merged image (Ad). Note that 4.1B is enriched at the paranodes and JXPs, and uniformly at lower levels throughout the axon. (B) Caspr ${ }^{-1}$ sciatic nerves immunostained against 4.1B (Ba, red), Caspr (Bb, green), AnkG (Bc, blue) and merged image (Bd). Note that 4.1B enrichment is lost at the paranodal/juxtaparanodal regions. It is uniformly expressed along the length of the axon. (C) Wild-type sciatic nerves immunostained against 4.1B (Ca, red,), $\mathrm{K}_{\mathrm{V}} 1.1$ (Cb, green), nodal NF186 (Cc, blue) and merged image (Cd). (D) Caspr-/- sciatic nerves immunostained against 4.1B (Da, red), KV1.1 (Db, green), NF186 (Dc, blue) and in merged image (Dd). Note that 4.1B is localized evenly along the length of the axon, and $\mathrm{K}_{\mathrm{V}} 1.1$ has re-localized to the paranodes, as has been seen in all paranodal AGSJ mutants. (E) Wild-type sciatic nerves immunostained against 4.1B (Ea, red), Nfasc (Eb, green), NF186 (Ec, blue), and in merged image (Ed). (F) $\mathrm{Cgt}^{-/}$sciatic nerves immunostained against 4.1B (Fa, red), Nfasc (Fb, green), NF186 (Fc, blue), and in merged image (Fd). Note that 4.1B is uniformly expressed along the axon and paranodal NF155 is disrupted at the paranodes. 


\subsection{Discussion}

The organization of specialized membrane environments that create cellular diversification depends on linkage of cellular membranes with the underlying cytoskeleton (Jekely, 2007). The 4.1 protein family functions to link the plasma membrane with the actin cytoskeleton (Chen et al., 2005; Hoover and Bryant, 2000; Sun et al., 2002). In the nervous system, the cell-adhesion molecules, Caspr and Caspr2 contain 4.1-binding sites, which are required for their localization to the paranodes and JXPs, respectively (Horresh et al., 2010; Poliak et al., 2003; Traka et al., 2003). When Caspr expression is genetically ablated, AGSJs fail to form, leading to loss of axonal domain segregation and disrupted paranodal axonal cytoskeleton (Bhat et al., 2001; Garcia-Fresco et al., 2006), suggesting that paranodal AGSJs are linked to axonal cytoskeleton. In this study, phenotypic analyses of $4.1 B$ mutants showed that $4.1 \mathrm{~B}$ is required for the stability of paranodal AGSJs in myelinated axons.

\subsubsection{Isoform Specific Functions of 4.1B in PNS and CNS Myelinated Axons}

Phenotypic analysis of $\beta$-Act-Cre $; 4.1 B^{\text {Flox }}$ mutant sciatic nerves showed paranodal defects as early as P30. The CNS paranodal defects at P30 were relatively mild, but at P90 transverse septa were often lost and paranodal loops coming off the axolemma, indicating an important role for 4.1B in AGSJ stabilization. Phenotypic analysis of the previously reported $4.1 B$ mutants (Yi et al., 2005) did not show disorganized Caspr localization or loss of paranodal septa in the PNS at P90, but revealed progressive destabilization of CNS paranodal septa at later stages of development. These variable phenotypes between $4.1 B$ mutants could be attributed to the nature of $4.1 B$ mutations, their genetic 
backgrounds or the multiple splice isoforms of 4.1B. Strain genetic backgrounds could be a contributing factor to phenotypic discrepancies between the two $4.1 B$ mutants. The $4.1 B^{-/}$mice are on a $C 57 B L 6$ background, while the $\beta$-Act-Cre $; 4.1 B^{F l o x}$ mice are on a mixed background of C57BL6 and $129 \mathrm{SvEv} . \beta$-Act-Cre; $4.1 B^{\text {Flox }}$ mice show a consistent phenotype throughout all mixed lines. We also examined the localization of 4.1B, Caspr, AnkG, $\mathrm{K}_{\mathrm{V}} 1.2$, and NF186 in each background strain and did not observe any disruptions in the localization of these proteins or the morphology of the paranodes (Fig. 2.8). These results suggest that the paranodal phenotypes observed in $\beta$-Act-Cre; $4.1 B^{\text {Flox }}$ mice are due to loss of 4.1B and not due to genetic background of the strains used.

In agreement with earlier studies that showed two 4.1B isoforms in brain lysates (145 $\mathrm{kDa}$ and 125kDa) (Denisenko-Nehrbass et al., 2003), we also observed the same isoforms in the spinal cord. Differential alternative splicing in the PNS versus CNS may allow 4.1B to function in a tissue-specific manner. The expression of close to 8 different isoforms in the PNS may explain the variation in phenotype seen between PNS and CNS, as well as the differences between $\beta$-Act-Cre; $4.1 B^{F l o x}$ and $4.1 B^{-\alpha}$ mutants. The phenotypic differences may lie in the nature of the $4.1 B$ mutations, as specific isoforms may play different roles in domain organization and stability in the CNS and the PNS. In $4.1 B^{-/-}$ mutants, exon 3 was disrupted by insertion of a puromycin resistance cassette (Yi et al., 2005). It is possible that some compensatory isoforms or splice variants remain in $4.1 B^{-/}$ mutants, which may not be detected by our antibodies directed to the U3 region of 4.1B, or by antibodies directed to the U2 region of 4.1B (Kang et al., 2009; data not shown). We believe that our targeted deletion of a common exon 6 , which encodes the middle portion of the FERM domain, generates a $4.1 B$ null mutation. Further evidence for a $4.1 B$ 
null mutation lies in the strong paranodal disorganization phenotype displayed by $\beta$-ActCre $; 4.1 B^{F l o x}$ compared to that observed in $4.1 B^{-/}$mutants, even at one year of age. In addition, our immunohistochemical, immunoblot, and RT-PCR analyses did not reveal compensatory expression of $4.1 \mathrm{R}$ at the paranodes, or an increase in $4.1 \mathrm{R}$ in the sciatic nerves of $4.1 B$ mutants, as was reported in Horresh et al. (2010). Together, our data demonstrate that 4.1B is critically required for the stabilization of the paranodal AGSJs and the juxtaparanodal axo-glial scaffolds in myelinated axons.

\subsubsection{Organization of Axonal Domains and Localization of 4.1B}

A key question that remains unresolved is whether 4.1B is required in the formation of paranodal and juxtaparanodal domains, or for their stability. In the absence of 4.1B, paranodal AGSJs are formed (Figs. 2.5 and 2.6), suggesting that 4.1B is not required for the formation, but for the stability of AGSJs (Bhat et al., 2001; DenisenkoNehrbass et al., 2003; Horresh et al., 2010; Poliak et al., 2003). Furthermore, disruption of paranodal AGSJs in Caspr and Cgt mutants (Fig. 2.9), as well as Cont (Gollan et al., 2002) mutants results in diffuse localization of $4.1 \mathrm{~B}$ along the axon, suggesting that $4.1 \mathrm{~B}$ requires AGSJs to become enriched at these domains. Paranodal localization of 4.1B may also be aided through its interactions with $\alpha \mathrm{II}$ and $\beta \mathrm{II}$ spectrin, which are enriched at the paranodal region (Garcia-Fresco et al., 2006; Ogawa et al., 2006). These observations indicate that paranodal and juxtaparanodal proteins initially cluster independently of 4.1B, and once clustered through axon-glial interaction mechanisms, 4.1B interactions ensure that these axo-glial adhesion complexes are stabilized. 


\subsubsection{Role of Axonal Cytoskeleton in Stabilizing Axon-Glial Interactions and}

\section{Septate Junctions}

The molecular identities of the complexes responsible for stabilizing axonal domains over the life of the axon are not well understood. At the paranodes, AGSJs are

organized by interactions between Caspr, Cont and Nfasc ${ }^{\mathrm{NF} 155}$ (Bhat et al., 2001; Boyle et al., 2001; Pillai et al., 2009). Loss of Caspr leads to disorganization of the paranodal region and axonal cytoskeleton, suggesting that AGSJs are linked to the axonal cytoskeleton (Garcia-Fresco et al., 2006; Ogawa et al., 2006). Paranodal disorganization in $\beta$-Act-Cre $; 4.1 B^{\text {Flox }}$ mutants provides further evidence that AGSJs are linked to axonal cytoskeleton and that 4.1B is required to ensure stability of the paranodal region. Support for the role of $4.1 \mathrm{~B}$ as a cytoskeletal adaptor protein comes from transgenic and rescue studies that show Caspr lacking its 4.1-binding domain is able to cluster at paranodes, but fails to establish a barrier to prevent juxtaparanodal components from entering paranodal space (Horresh et al., 2010). Further, Caspr with a deleted C-terminus can reach, but fails to stabilize at paranodes, suggesting that the C-terminus is essential for Caspr stabilization (Gollan et al., 2002). Protein 4.1B likely stabilizes the paranodes through direct interactions with Caspr and other cytoskeletal proteins such as AnkB and $\alpha$ II- and $\beta I I-s p e c t r i n$ (Garcia-Fresco et al., 2006; Ogawa et al., 2006). Interestingly, domain disorganization in $4.1 B$ mutants did not result in conduction velocity differences in P30 wild type, $\beta$-Act-Cre $; 4.1 B^{\text {Flox }}$, or $4.1 B^{-/}$sciatic nerve fibers (data not shown). In addition, neither $4.1 B$ mutants display obvious behavioral deficits, as of 4 months of age.

However, precise electrophysiological recordings of individual axons and behavioral tests for motor function could reveal deficits in older $4.1 B$ mutants. 
The JXP is established by interactions between Caspr2 and TAG-1 (Poliak et al., 2003; Traka et al., 2003). In this region no axo-glial junctions are formed, but a loose axo-glial scaffold is established that moves towards the paranode in paranodal mutants (Bhat et al., 2001; Boyle et al., 2001; Dupree et al., 1999; Pillai et al., 2009). These findings suggest that the JXP is not anchored with axonal cytoskeleton even though Caspr2 also has 4.1B-binding sequences (Denisenko-Nehrbass et al., 2003; Horresh et al., 2010). The juxtaparanodal region clusters potassium channels, which are lost in Caspr2 and Tag- 1 mutants (Poliak et al., 2003; Traka et al., 2003). Interestingly, both the $\beta$-ActCre $; 4.1 B^{\text {Flox }}$ and $4.1 B^{-/-}$mutants revealed disorganization of juxtaparanodal proteins, with loss of potassium channel and Caspr2 localization. This suggests that formation and/or stabilization of the juxtaparanodal axo-glial scaffold, including clustering of potassium channels, critically depends on 4.1B, thus highlighting the role of axonal cytoskeletal adaptor proteins in the stability of axonal domains.

\subsubsection{Evolutionary Role of Cytoskeletal Proteins in Stabilizing Axo-glial Septate junctions}

The founding member and Drosophila ortholog of the vertebrate Caspr family, Neurexin IV (Nrx IV), contains a 4.1-binding sequence in its C-terminus (Banerjee et al., 2006b; Baumgartner et al., 1996; Bellen et al., 1998; Bhat, 2003; Bhat, 2004). An ortholog of the vertebrate 4.1 family that co-localizes with Nrx IV at septate junctions is Coracle (Cora) (Fehon et al., 1994). Null mutants of $n r x I V$ and cora show mislocalization of Cora and Nrx IV, respectively, and fail to establish septate junctions (Banerjee et al., 2006a; Baumgartner et al., 1996; Fehon et al., 1994; Lamb et al., 1998; 
Ward et al., 1998). Furthermore, mutations in the FERM domain of Cora disrupt its membrane localization, suggesting that the interaction between Cora and its transmembrane-binding partners is required for localization of Cora at the membrane. Together, our data provide convincing evidence that 4.1B is critically required for proper maintenance of axonal domains in myelinated axons by acting as a molecular adaptor between membrane components at the paranodal and juxtaparanodal regions and the underlying axonal cytoskeleton. These interactions are central to the long-term stability of axonal domains and thus saltatory nerve conduction. 


\section{Chapter 3}

\section{Pinceau Organization in the Cerebellum Requires Distinct Functions of Neurofascin in Purkinje and Basket Neurons During Postnatal Development}

\subsection{Introduction}

Development of neuronal circuits is controlled by signaling mechanisms that coordinate precise targeting of presynaptic axons to their postsynaptic targets (Kolodkin and Tessier-Lavigne, 2011; Lu et al., 2009). The GABAergic interneurons frequently target inhibitory synapses to the soma or AIS of principal cells (Ango et al., 2004; Freund and Buzsaki, 1996; Somogyi et al., 1998). In the cerebellum, basket interneurons target the Purkinje soma and AIS (Ango et al., 2004; Li et al., 1992; Somogyi and Hamori, 1976; Somogyi et al., 1983). Since Purkinje neurons are the sole cerebellar output source, their activity must be strictly regulated. Genetic mutations resulting in Purkinje neuron dysfunction lead to cerebellar dysfunction and ataxia (Gold et al., 2003; Hamilton et al., 1996; Levin et al., 2006; Perkins et al., 2010; Rinaldo and Hansel, 2010; Wang and Zoghbi, 2001). Cerebellar basket axons branch into many axonal collaterals that form the basket-shaped pinceau at the Purkinje soma/AIS (Ramon y Cajal, 1911). The pinceau inhibitory synapses modulate the frequency of Purkinje neuron action potential spikes (Sakaba, 2008). However, the mechanisms responsible for the organization of the pinceau and targeting of basket axon collaterals to Purkinje soma/AIS are not well understood. 
The Purkinje AIS is enriched in immunoglobulin-like domain-containing CAMs, including Nfasc (Brummendorf et al., 1998; Huang, 2006), which bind to AnkG, an adaptor protein that links membrane proteins to the spectrin/actin cytoskeleton (Bennett and Baines, 2001; Boiko et al., 2007; Zhou et al., 1998). Other components of the Purkinje AIS required for action potential initiation are the voltage gated ion channels, including sodium $\left(\mathrm{Na}_{\mathrm{V}}\right)$ channels (Benton and Raman, 2009; Catterall, 1981; Khaliq and Raman, 2006; Raman and Bean, 1999). The mechanisms that underlie the enrichment and maintenance of ion channels at the AIS are still poorly understood.

Previous studies showed that loss of AnkG from the Purkinje AIS resulted in mislocalization of Nfasc, altered basket axon targeting and disrupted pinceau synapses (Ango et al., 2004). Purkinje neuron expression of dominant negative Nfasc also disrupted pinceau synapses implicating a role for Nfasc in the formation of GABAergic pinceau synapses (Ango et al., 2004). Recent studies used an inducible Cre recombinase to ablate Nfasc in adult neurons and revealed slow destabilization of the Purkinje AIS, causing deficits in Purkinje physiology (Zonta et al., 2011). However, these studies did not address the role of Nfasc in the developmental organization of the Purkinje AIS or basket axon targeting to form the pinceau.

Here, we show that Purkinje neuron-specific ablation of Nfasc results in failure of the Purkinje AIS to mature and basket axon collaterals fail to establish proper pinceau at the Purkinje soma/AIS. Consequently, basket neuron inhibitory input to Purkinje neurons is significantly reduced. Further, Nfasc ablation in both Purkinje and basket neurons revealed Nfasc expression in basket neurons and caused abnormal basket axon collateral branching and targeting to the Purkinje soma/AIS, resulting in more severe pinceau 
disorganization. Together, our results establish that Nfasc performs distinct functions in Purkinje and basket neurons to coordinate cerebellar pinceau organization during postnatal development.

\subsection{Materials and Methods}

\subsubsection{Animals}

All animal experiments were carried out according to UNC-IACUC approved guidelines for ethical treatment of laboratory animals. Parv-Cre and Tau ${ }^{m G F P / L a c Z}$ mice (data not shown) (Hippenmeyer et al., 2005) were generously provided by Dr. William Snider (University of North Carolina). Pcp2-Cre mice (Barski et al., 2000) and $\beta$-ActinCre mice (Thaxton et al., 2011) were obtained from Jackson Labs (Maine, USA). Gad67GFP transgenic mice (Ango et al., 2004) were generously provided by Dr. Patricia Maness (University of North Carolina).

\subsubsection{Tissue specific deletion of the Nfasc Gene}

The $N f a s c^{F l o x}$ mice utilized in this study were previously described (Pillai et al., 2009; Thaxton et al., 2011). Genomic DNA was extracted from tail and cerebellar samples using the REDExtract-N-Amp Tissue PCR kit (Sigma-Aldrich, USA). The primer sequences that were used to identify specific genotypes were: Pcp2-Cre 5'GGCCAATGTCTGACCAAATAC -3' and 5'- CTCCCACCGTCAGTACGTGAGAT3'; Parv-Cre 5'- CAGCCTCTGTTCCACATACACTCC- 3' and 5'TCACTCGAGAGTACCAAGCAGGCAGGAGATATC- 3'; Tau ${ }^{m G F P / L a c Z} 5$ '- 
$3^{\prime}$.

\subsubsection{Antibodies}

The following antisera were previously described: Guinea pig anti-NF186 and rat anti-pan Nfasc (Pillai et al., 2009; Thaxton et al., 2010), rat anti-AnkG (Thaxton et al., 2010), and rabbit anti-Brevican (John et al., 2006). Other primary antibodies used here include: rabbit anti-Calbindin (Calb) and mouse anti-Calbindin (Sigma), mouse antiParvalbumin (Parv) (Millipore) and mouse anti-phospho-neurofilaments (pNfl) (Covance, SMI 31). The monoclonal antibodies mouse anti-Nav1.6 (K87A,10), mouse anti- $\mathrm{K}_{\mathrm{V}} 1.1$ (K20/78), mouse anti-K $\mathrm{V}_{\mathrm{V}} 1.2(\mathrm{~K} 14 / 16)$ and mouse anti-PSD95 (K28/43) were obtained from UC Davis/NIH NeuroMab Facility. Secondary antibodies used for immunofluorescence were: Alexa Fluor conjugated -488, -568, and -647 (Molecular Probes, Invitrogen).

\subsubsection{Tissue Preparation and Immunostaining}

Tissues used for immunostaining were prepared essentially as previously

described (Buttermore et al., 2011). Briefly, for cerebellar sections, wild type and mutant mice of either gender were deeply anesthetized and transcardially perfused with saline buffer followed by ice cold 4\% paraformaldehyde in PBS. The cerebellum was dissected out and post-fixed in $4 \%$ paraformaldehyde overnight at $4{ }^{\circ} \mathrm{C}$. The tissues were rinsed several times in PBS and sectioned to $30 \mu \mathrm{m}$ using a Vibratome (Leica). The cerebellar sections were then immediately immunostained, beginning with permeabilization in ice- 
cold acetone for 20min followed by washing in PBS and blocking in buffer (5\% BSA, $1 \%$ NGS, and $0.2 \%$ Triton X-100, in PBS) for $1 \mathrm{hr}$. Cerebellar sections were then incubated with primary antibodies overnight in blocking buffer, followed by washing in PBS, incubation with secondary antibodies for $1 \mathrm{hr}$ at room temperature (RT) and another round of washing in PBS. Sections were mounted in VectaShield (Vector Labs) before imaging.

For $\mathrm{Na}_{\mathrm{V}}$ antibodies, wild type and mutant mice, of either gender, were deeply anesthetized and transcardially perfused with saline buffer followed by ice cold $2 \%$ paraformaldehyde in PBS. The cerebellum was dissected out and post-fixed in $2 \%$ paraformaldehyde for two hours on ice. The tissues were rinsed several times in PBS before being placed in $30 \%$ sucrose overnight for two nights. The tissues were then blocked and frozen in M-1 Embedding Matrix (Shandon, Thermo Scientific) and sectioned to $20 \mu \mathrm{m}$ using a cryostat (Bright Instrument Company LTD). The cerebellar sections were then immediately immunostained as described above.

\subsubsection{Quantification and analysis}

Quantification of basket axon collaterals at the Purkinje soma and AIS at P20 was completed utilizing three independent wild type, Pcp2-Cre;Nfasc ${ }^{\text {Flox }}$ and ParvCre;Nfasc ${ }^{\text {Flox }}$ age-matched mice of either gender. Cerebellar tissue was processed as described above and immunostaining with pNfl and Calb allowed visualization of basket axon collaterals surrounding the Purkinje soma and AIS. Purkinje neurons from each mouse were selected at random and the number of basket axon collaterals surrounding 
the Purkinje soma and AIS were counted. The average number of basket collaterals was calculated for each genotype, age and location (soma versus AIS).

Quantification of compact versus broken/non-compact pinceau, immunostained by $\mathrm{K}_{\mathrm{V}} 1.2$ channels at $\mathrm{P} 16, \mathrm{P} 20$ and $\mathrm{P} 30$ was completed utilizing three independent wild type, Pcp2-Cre;Nfasc ${ }^{\text {Flox }}$, and Parv-Cre;Nfasc ${ }^{\text {Flox }}$ age-matched mice of either gender. Cerebellar tissue sections were immunostained with $\mathrm{K}_{\mathrm{V}} 1.2$ and Calb, and Purkinje neurons were randomly selected to score and count the pinceau phenotype. In addition, the presence of ectopic $K_{V} 1.2$ clusters around the Purkinje soma was also quantified for each genotype.

Neurons were selected randomly for both pNfl and $\mathrm{K}_{\mathrm{V}} 1.2$ quantification by looking at the immunostaining in the Calb channel alone and at the Purkinje AISs. If a full Purkinje AIS was visible, a confocal image was taken of both Calb and the other channel for counting ( $\mathrm{pNfl}$ or $\mathrm{K}_{\mathrm{V}} 1.2$ ). Images were then used for quantitation.

Quantification of synapse formation on the bottom half of the Purkinje soma closest to the granule-cell layer of one-month-old wild type, Pcp2-Cre;Nfasc ${ }^{\text {Flox }}$ and Parv-Cre; Nasc $^{\text {Flox }}$ cerebella was completed utilizing three separate EM grids from one mouse of each genotype and of either gender. The average number of synapses per Purkinje soma-base was calculated.

Quantification of Purkinje neuron death in 5 month-old Parv-Cre;Nfasc ${ }^{\text {Flox }}$ compared to wild type cerebella was completed by immunostaining cerebellar sections with Calb to elucidate Purkinje neurons. The total number of Purkinje neurons present in each of the following cerebellar loops: I, II, III, IV, V, VIa, VIb, and VIII were counted 
in five independent mice of either gender per genotype. The average number of Purkinje neurons per loop was calculated for each genotype.

In the quantification of pNfl (Fig. 3.5), pinceau $\mathrm{K}_{\mathrm{V}} 1.2$ channels (Fig. 3.5) and EM synapse counting (Fig. 3.8) "n" refers to the number of Purkinje cells/pinceau counted. The cells were counted from 3-4 separate animals for each genotype. In counting Purkinje neuron death (Fig. 3.12), "n" refers to the number of cerebellar loops that were counted from five separate animals for each of the three genotypes. For statistical analysis, we used the Student's t-test (unpaired) and ANOVA test (single factor).

\subsubsection{Transmission Electron Microscopy (TEM)}

TEM of age-matched wild type, Pcp-2-Cre; Nfasc $^{\text {Flox }}$ and Parv-Cre;Nfasc ${ }^{\text {Flox }}$ mice of either gender was carried out essentially as described previously (Garcia-Fresco et al., 2006; Pillai et al., 2009; Thaxton et al., 2010).

\subsubsection{Image analysis}

Confocal images were captured with a BioRad Radiance 2000 laser-scanning system attached to a Zeiss Axioplan2 microscope. Scanning parameters were optimized for wild type tissues and maintained for scanning the mutant tissues. The immunofluorescence images shown are Z stacks of 4-8 sections with a scan step of $0.25 \mu \mathrm{m}$. Adobe Photoshop software was used for processing and assembling of all figures. 


\subsubsection{Electrophysiology}

Slice preparation:

Experiments were performed on both wild type and Pcp2-Cre;Nfasc ${ }^{\text {Flox }}$ mutant littermate mice, aged between 3 months and 6 months. At these developmental time points Purkinje neuron degeneration is not observed in Pcp2-Cre; fasc $^{\text {Flox }}$ mutants. Animals were first anesthetized with halothane and then rapidly decapitated. The cerebellar vermis was immediately removed and cooled to $4^{\circ} \mathrm{C}$ in artificial CSF (ACSF) containing (in mM): $124 \mathrm{NaCl}, 5 \mathrm{KCl}, 1.25 \mathrm{Na}_{2} \mathrm{HPO}_{4}, 1 \mathrm{MgSO}_{4}, 2 \mathrm{CaCl}_{2}, 26 \mathrm{NaHCO}_{3}$ and $10 \mathrm{D}$-glucose, bubbled with $95 \% \mathrm{O}_{2}$ and $5 \% \mathrm{CO}_{2}$. Parasagittal slices of the cerebellar vermis $(190 \mu \mathrm{m})$ were prepared with a vibratome (VT-1000S; Leica). Slices were then incubated at least $1 \mathrm{hr}$ at RT in oxygenated ACSF.

Somatic whole cell patch-clamp recordings:

Throughout recording, slices were continuously perfused with ACSF, at room temperature to improve the quality of clamp. Patch-clamp recordings from the Purkinje soma were performed using an EPC-10 amplifier (HEKA Electronics, Lambrecht/Pfalz, Germany). Currents were filtered at $3 \mathrm{kHz}$, digitized at $5-10 \mathrm{kHz}$, and acquired using Patchmaster software (HEKA). For recording action potentials, patch pipettes (2-5 M 2$)$ were filled with a solution containing (in mM): $120 \mathrm{~K}$-gluconate, $9 \mathrm{KCl}, 10 \mathrm{KOH}, 3.48$ $\mathrm{MgCl}_{2}, 10 \mathrm{HEPES}, 4 \mathrm{NaCl}, 4 \mathrm{Na}_{2} \mathrm{ATP}, 0.4 \mathrm{Na}_{3} \mathrm{GTP}$, and 17.5 sucrose (pH 7.25-7.35). For spontaneous inhibitory postsynaptic current (sIPSC) and mini inhibitory postsynaptic current (mIPSC) recordings, the internal solution contained (in $\mathrm{mM}$ ): $150 \mathrm{CsCl}, 4.6$ $\mathrm{MgCl}_{2}, 10 \mathrm{HEPES}, 1$ EGTA, $0.1 \mathrm{CaCl}_{2}, 4 \mathrm{Na}_{2} \mathrm{ATP}, 0.4 \mathrm{Na}_{3} \mathrm{GTP}$. Purkinje neurons were 
voltage-clamped at holding potentials in the range of -65 to $-70 \mathrm{mV}$. At this potential and with the CsCl-based internal solution, excitatory postsynaptic currents (EPSCs) and IPSCs were recorded as inward currents. To specifically isolate the excitatory inward currents from inhibitory inward current, NBQX $(10 \mu \mathrm{M})$ and APV $(50 \mu \mathrm{M})$ were added to the ACSF throughout the sIPSCs and mIPSCs recordings in order to block AMPA and NMDA receptors. mIPSCs, corresponding to responses to single synaptic release, were measured under action potential suppression by $1 \mu \mathrm{M}$ TTX, a voltage-dependent $\mathrm{Na}+$ channel blocker. For EPSPs/EPSCs recordings with the K-Gluconate based internal solution, Picrotoxin $(200 \mu \mathrm{M})$ was added in the ACSF throughout recordings. To activate PFs, glass electrodes filled with ACSF were placed in the upper molecular layer. The input and series resistances were monitored throughout experiments by applying hyperpolarizing voltage steps $(-10 \mathrm{mV})$ at the end of each sweep. Recordings were excluded if series or input resistances varied by $>15 \%$ over the course of the experiments.

Analysis

Data were analyzed with Excel (Microsoft) or Igor (Wavemetrics). sIPSCs and mIPSCs were analyzed using Patcher's Power Tool program with a threshold of 10pA for events detection. All data are expressed as the mean +/- S.E.M. For statistical analysis, we used the Student's t-test (paired/unpaired) and the Mann-Whitney U test, when appropriate. 


\subsubsection{Behavioral testing}

Ten wild type and ten Pcp2-Cre;Nfasc ${ }^{F l o x}$ mice of either gender were tested at one month, two months, three months and four months in the following tests.

\section{Locomotor activity}

Exploratory activity in a novel environment was assessed by one-hour trials in an open field chamber $(40 \mathrm{~cm} \times 40 \mathrm{~cm} \times 30 \mathrm{~cm}$ ) crossed by a grid of photobeams (VersaMax system, AccuScan Instruments). Counts were taken of the number of photobeams broken during the trial in five-minute intervals, with separate measures for ambulation (total distance traveled) and rearing movements.

Rotarod

Mice were tested for motor coordination and learning on an accelerating rotarod (Ugo Basile, Stoelting Co., Wood Dale, IL). For each test session, animals were given two trials, with 45 seconds between each trial. Rpm (revolutions per minute) was set at an initial value of 3 , with a progressive increase to a maximum of $30 \mathrm{rpm}$ across five minutes (the maximum trial length). Measures were taken for latency to fall from the top of the rotating barrel.

\subsubsection{Basket cell dye-injections}

Parasagittal slice preparation

Mice were anesthetized with pentobarbital $(40 \mathrm{mg} / \mathrm{kg})$ and decapitated after disappearance of corneal reflexes, in compliance with University of North Carolina 
guidelines. Brains were rapidly removed and immersed in ice-cold dissection buffer (in mM: $87 \mathrm{NaCl}, 2.5 \mathrm{KCl}, 1.25 \mathrm{NaH}_{2} \mathrm{PO}_{4}, 26 \mathrm{NaHCO}_{3}, 75$ sucrose, 10 dextrose, 1.3 ascorbic acid, $7 \mathrm{MgCl}_{2}$, and $0.5 \mathrm{CaCl}_{2}$ ) bubbled with $95 \% \mathrm{O}_{2}-5 \% \mathrm{CO}_{2}$. The cerebellum was dissected and $250 \mu \mathrm{m}$ parasagittal slices were prepared using a vibrating microtome (Leica VT1000S). Slices were allowed to recover for $20 \mathrm{~min}$ in a $35^{\circ} \mathrm{C}$ submersion chamber filled with oxygenated artificial cerebrospinal fluid (ACSF) (in mM; $124 \mathrm{NaCl}$, $3 \mathrm{KCl}, 1.25 \mathrm{Na}_{2} \mathrm{PO}_{4}, 26 \mathrm{NaHCO}_{3}, 1 \mathrm{MgCl}_{2} 2 \mathrm{CaCl}_{2}$ and 20 glucose) and then kept at room temperature for $>40$ min until use.

\section{Biocytin fills of cerebellar basket neurons}

Slices were placed in a submersion chamber, maintained at $30^{\circ} \mathrm{C}$ and perfused at 2 $\mathrm{mL} / \mathrm{min}$ with oxygenated ACSF (as described above). Cells were visualized using a Ziess Axioskop microscope equipped with infrared differential interference contrast (IR-DIC) optics. Patch pipettes were pulled from thick-walled borosilicate glass (P2000, Sutter Instruments Novato, CA). Open tip resistances were between 2.5-5 $\mathrm{M} \Omega$ when pipettes were filled with the internal solution containing (in mM): $100 \mathrm{~K}$-Gluconate, $20 \mathrm{KCl}, 0.2$ EGTA, 4 Mg-ATP, 0.3 Na-GTP, 10 HEPES, 10 Na-phosphocreatine, 0.5\% neurobiotin (Vector Labs) and 0.025 Alexa-488 or 568 with $\mathrm{pH}$ adjusted to 7.25 with $1 \mathrm{M} \mathrm{KOH}$ and osmolarity adjusted to $\sim 295$ mOsm by addition of sucrose. Voltage-clamp recordings were performed in the whole-cell configuration using patch-clamp amplifier (Multiclamp 700A, Molecular Devices). Pipette seal resistances were > $1 \mathrm{G} \Omega$, and pipette capacitive transients were minimized prior to breakthrough. Basket neurons were filled for 15-20 min before the slice was removed and placed in $4 \%$ paraformaldehyde (in $0.1 \mathrm{M}$ PBS) 
overnight. The cerebellar sections were processed for immunostaining as described above.

\section{Quantification and analysis}

Two independent wild type, Pcp2-Cre;Nfasc ${ }^{\text {Flox }}$, and Parv-Cre;Nfasc ${ }^{\text {Flox }}$ mice of either gender at P10 and P20 were utilized for biocytin dye-injection experiments. A minimum of three basket cells were injected in three cerebellar sections from each genotype. Healthy looking basket neurons were randomly selected for injections based on their location above the Purkinje neurons. All clearly stained basket cells were imaged and z-stacks merged. The number of primary branches forming off the main axon for each basket neuron were counted and averaged for each genotype and age and "n" refers to the number of basket cells counted for each genotype. For statistical analysis, we used the Student's t-test (unpaired) and ANOVA test (single factor).

\subsection{Results}

\subsubsection{Developmental Organization of the Cerebellar Pinceau}

The cerebellar interneurons migrate through the white matter tracks of the cerebellum before moving in the vicinity of the Purkinje neuron layer and differentiating into basket and stellate interneurons in the molecular layer (Ango et al., 2004; Palay and Palay, 1974; Sudarov et al., 2011; Zhang and Goldman, 1996). Axon collaterals from 5-7 basket neurons contribute to the formation of the pinceau, and each basket neuron can innervate several Purkinje neurons, allowing for precise gating of Purkinje neuron output. To establish the developmental organization and localization of various molecular 
components at the pinceau, we utilized a combination of molecular markers to follow the developmental timeline of pinceau organization, including the unique enrichment of potassium channels at the core of the pinceau. We carried out immunostaining of wild type cerebellar sections against Parv to label both Purkinje neurons and molecular layer interneurons, including basket neurons (Bastianelli, 2003). We also immunostained for Calb as a specific marker for Purkinje neurons (Nordquist et al., 1988), phosphorylated neurofilaments ( $\mathrm{pNfl}$ ) as a marker for basket neuron collaterals, and potassium channels $\left(K_{V} 1.2\right)$ to label the core of the pinceau formed by basket axon terminals that target the Purkinje AIS. As shown in Fig. 3.1, co-immunostaining against Parv and Calb at P10 shows Parv expression in basket neurons (b) (Fig. 3.1Aa-b) in the vicinity of the Purkinje neurons. Note that Parv is also expressed in Purkinje neurons (merged yellow color) (Fig. 3.1 Ab), but Calb is not expressed in basket neurons. At this stage, Parv immunoreactivity at the developing pinceaux is not expanded (Fig. 3.1Ab, arrow). Triple immunostaining of $\mathrm{P} 10$ cerebellar sections using antibodies against Calb, $\mathrm{pNfl}$ and $\mathrm{K}_{\mathrm{V}} 1.2$ showed that basket neuron collaterals are beginning to target the Purkinje AIS (Fig. 3.1Bb, merged image Fig. 3.1Bd, arrow), but the $\mathrm{K}_{\mathrm{V}} 1.2$ immunoreactivity is not yet detectable at the developing pinceau (Fig. 3.1Bc, arrowhead, merged image Fig. 3.1Bd). At P12, basket neuron collaterals reach the Purkinje AIS, as visualized by both Parv (Fig. 3.1Ca) and pNfl (Fig. 3.1Db, arrowhead). At P12, $\mathrm{K}_{\mathrm{V}} 1.2$ begins to label the developing pinceau (Fig. 3.1Dc, arrowhead) around the Purkinje AIS (merged image Fig. 3.1Dd). At P16, the structure of the pinceaux begins to take shape and Parv localization broadens around the Purkinje AIS (Fig. 3.1Ea, merged image Fig. 3.1Eb, arrow) and more basket axon collaterals begin to target the Purkinje AIS, as revealed by immunostaining against pNfl 
(Fig. 3.1Fb, arrowhead). $\mathrm{K}_{\mathrm{V}} 1.2$ also begins to show increasing enrichment at the pinceaux (Fig. 3.1Fc, arrowhead). By P20, the pinceaux takes the characteristic coneshape and its development is almost complete, as revealed by Parv and pNfl localization (Fig. 3.1Ga, b, arrow; Fig. 3.1Hb, arrowhead) and the unique enrichment of $\mathrm{K}_{\mathrm{V}} 1.2$ channels (Fig. 3.1Hc, arrowhead; merged image Fig. 3.1Hd, arrow). By P30, the general developmental organization of the pinceaux is complete (Fig. 3.1 Ia, b; Ja-d), but the pinceaux becomes larger and the density of $\mathrm{K}_{\mathrm{V}}$ channels increases by P30 to P90 (Fig. 3.1Ka, b; La-d) (Sotelo, 2008). We also analyzed BAC transgenic Gad67-GFP mice which express GFP in cerebellar interneurons, including basket neurons and some Purkinje neurons (Ango et al., 2004). Immunostaining of Gad67-GFP cerebella at various postnatal developmental stages using anti-GFP to label basket axon collaterals and anti-Calb further supported the developmental timeline of the pinceau organization as revealed by various antibody markers in the wild type cerebella (Fig. 3.1M-Q). Based on the above immunohistochemical analysis, we have schematized the organization of the cerebellar pinceaux during postnatal development (Fig. 3.1R). Together, our data reveal distinct features of pinceau development in which basket axon collaterals target the Purkinje soma and the AIS and only the inner core of the pinceaux becomes enriched with potassium channels, which surrounds the Purkinje AIS. 


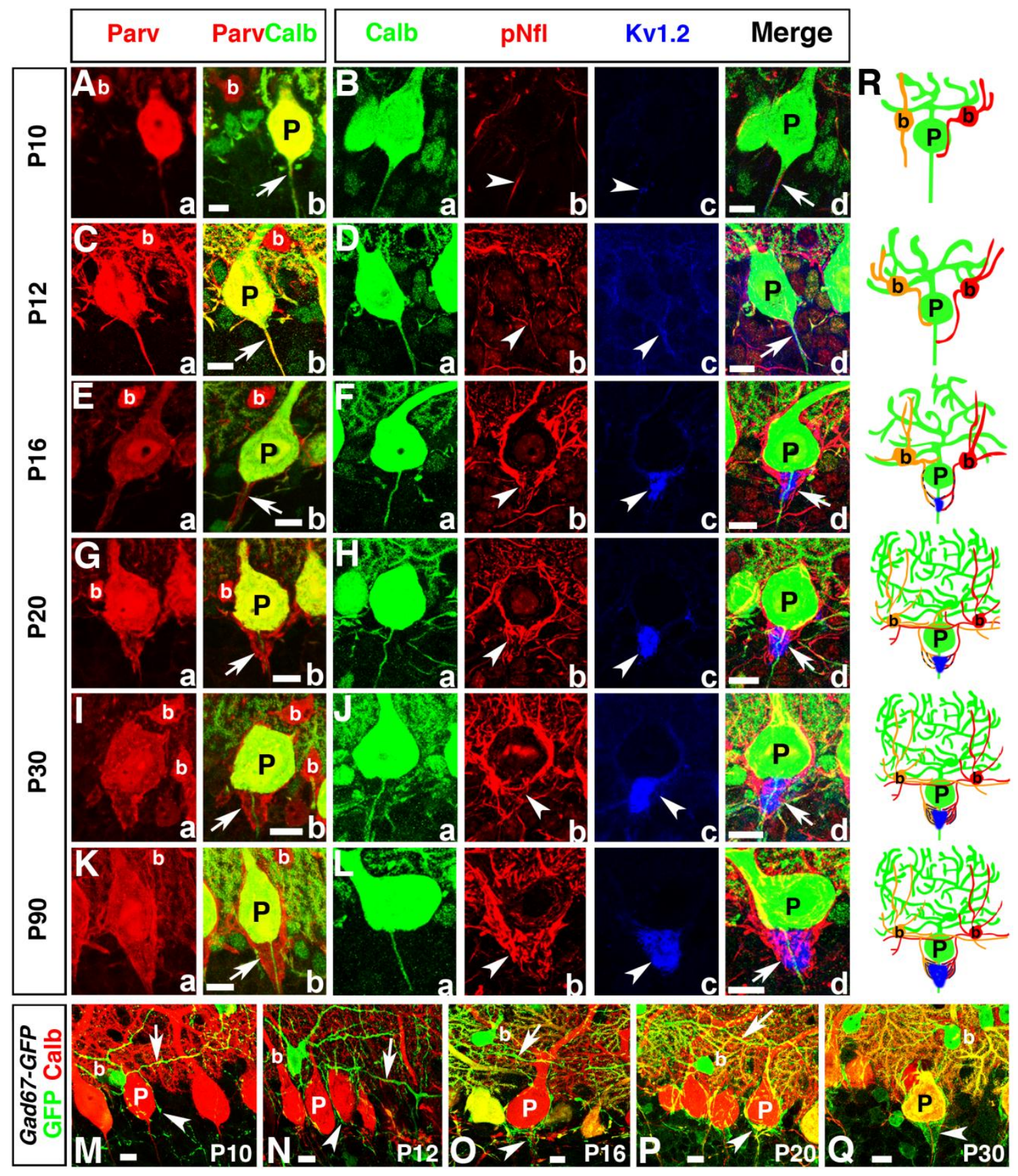

Figure 3.1 Molecular organization of the cerebellar pinceau during postnatal development.

Wild type cerebellar sections from ages P10-P90 (indicated on left) and immunostained against Parv, Calb, pNfl, and potassium channels ( $\left.\mathrm{K}_{\mathrm{V}} 1.2\right)$.

(A, B) At P10, very few basket neuron ("b"=basket neuron) collaterals have reached Purkinje neuron ("P") AIS's (arrows).

(C, D) At P12, a few basket neuron (b) collaterals have arrived at the Purkinje AIS (arrowhead) and faint traces of $\mathrm{K}_{\mathrm{V}} 1.2$ channels can be seen around the Purkinje AIS. 
(E, F) By P16, more basket neuron (b) collaterals surround the Purkinje (P) AIS and $\mathrm{K}_{\mathrm{V}} 1.2$ channels are enriched at the basket neuron terminals.

(G, H) At P20, the cone-shape of the pinceau is formed, as evident by Parv (Gb, arrow), pNfl (Hb, arrowhead, Hd, arrow) and $\mathrm{K}_{\mathrm{V}} 1.2$ clustering (Hc, arrowhead).

(I, J) At P30 the pinceau is fully mature ( $\mathrm{Ib}, \mathrm{Jd}$, arrows, $\mathrm{Jb}$, Jc arrowheads).

$(\mathrm{K}, \mathrm{L})$ At $\mathrm{P} 90$, the structure of the pinceau does not display major anatomical changes compared to P30 (Kb, Ld, arrows, Lb, Lc, arrowheads).

(M-Q) Cerebellar sections from Gad67-GFP BAC transgenic mice at ages P10 (M), P12 $(\mathrm{N})$, P16 (O), P20 (P) and P30 (Q) immunostained against GFP (green) and Calb (red).

The main basket axons (arrows) give off collaterals (arrowheads) that extend towards the Purkinje AIS begins at P10, (M, arrowhead) and form the cone-shape pinceau P30 (Q, arrowhead).

(R) Representative drawings of each stage of pinceau development. Purkinje neurons (P) in green, basket neurons (b) in red and orange and potassium channels in blue. Scale bars: $10 \mu \mathrm{m}$ 


\subsubsection{Nfasc is Expressed in Cerebellar Basket Axon Terminals at the Pinceau}

The anatomical features and the organization of the pinceau between basket axon collaterals and the Purkinje soma/AIS have raised fundamental questions about what precisely dictates the establishment of the pinceaux. Earlier studies showed that loss of AnkG at the Purkinje AIS led to mislocalization of Nfasc at the Purkinje AIS and disrupted organization of the pinceau, implicating Nfasc in pinceau organization (Ango et al., 2004). It was recently reported that loss of Nfasc in adult mice, using inducible Thyl$\mathrm{Cre}$, led to progressive disorganization of the Purkinje AIS and the pinceau (Zonta et al., 2011). While these studies highlight the role of Nfasc in Purkinje neurons for pinceau formation and AIS maintenance, it remains to be determined if Nfasc is functionally required in basket neurons during pinceau assembly. We addressed this issue by specifically ablating Nfasc in Purkinje, and in both Purkinje and basket neurons using cell type-specific Cre recombinase. To analyze the role of Purkinje neuron Nfasc in pinceau formation, we conditionally ablated Nfasc in Purkinje neurons using Purkinje-cell protein 2 (Pcp2)-Cre (Barski et al., 2000; Berrebi et al., 1991) and previously generated Nfasc ${ }^{\text {Flox }}$ mice (Pillai et al., 2009; Thaxton et al., 2011). We first confirmed the specificity of the Nfasc antibody by immunostaining cerebellar tissues from Actin-Cre; $\mathrm{NasC}^{\mathrm{Flox}}$ mice, which are null for Nfasc. This immunostaining showed that Nfasc is absent in ActinCre;Nfasc ${ }^{\text {Flox }}$ mice at P5, the age at which it clearly localizes to the Purkinje AIS in wild type cerebella (compare Fig. 3.2B to Fig. 3.2A). Further, we confirmed that Nfasc and AnkG co-localized at the Purkinje and basket AIS at P10 and P20 (Fig. 3.2C-D). We also confirmed that ablation of Nfasc in the cerebellum started from P0 using PCR analysis of Pcp2-Cre;Nfasc ${ }^{\text {Flox }}$ mice during postnatal development (Fig. 3.2E). 


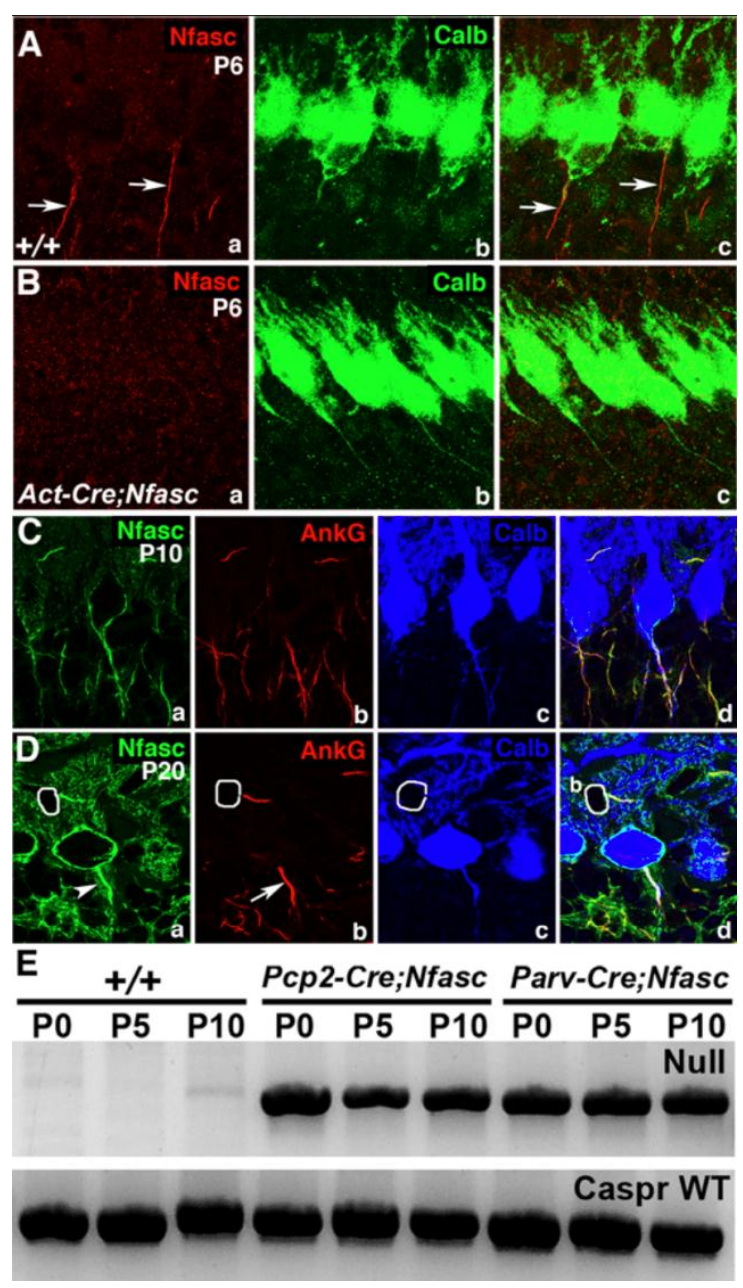

\section{Figure 3.2 Ubiquitous Ablation of Nfasc.}

Wild type (A) and Actin-Cre; Nfasc $^{\text {Flox }}$ (B) P5 cerebellar sections immunostained against Nfasc (a, red), Calb (b, green), and merged (c) show expression of Nfasc at the Purkinje AIS in wild type cerebella and complete loss of Nfasc in Actin-Cre; Nfasc ${ }^{\text {Flox }}$ cerebella, confirming the specificity of the Nfasc antibodies.

Wild type P10 (C) and P20 (D) cerebellar sections immunostained against Nfasc (a, green), AnkG (b, red), Calb (c, blue) and merged (d) show co-localization of Nfasc and AnkG at the Purkinje and basket AIS at P10, while Nfasc additionally begins to localize around the Purkinje soma. At P20, Nfasc and AnkG remain co-localized at the basket (b) and Purkinje AIS (Db, arrow), and Nfasc now extends around the entire Purkinje soma and is localized to the basket axon terminals surrounding the Purkinje AIS (Da, arrowhead).

(E) PCR amplification using primers to amplify the excision product of the Nfasc allele from wild type, Pcp2-Cre; fasc $^{\text {Flox }}$ and Parv-Cre;Nfasc ${ }^{\text {Flox }}$ cerebella (Thaxton et al., 2011). Primers to amplify wild type Caspr were used as a control. Note that Credependent recombination in the Nfasc locus is observed at P0 in Pcp2-Cre;Nfasc ${ }^{\text {Flox }}$ and Parv-Cre; Nfasc $^{\text {Flox }}$ cerebella. 
To ensure that Nfasc was specifically ablated in Purkinje neurons, we carried out immunostaining against $\mathrm{Nfasc}$ and Calb in wild type (+/+) and Pcp2-Cre;Nfasc ${ }^{\text {Flox }}$ mice during postnatal development. At P10, in wild type cerebella, Nfasc is enriched at the Purkinje AIS and slightly at the base of the Purkinje soma (Fig. 3.3Aa, arrow), as well as at the basket neuron (b) AIS (Fig. 3.3Aa, arrowhead; merged image Fig. 3.3Ab). In P10 Pcp2-Cre;Nfasc ${ }^{\text {Flox }}$ cerebella, Nfasc is undetectable at the Purkinje cell AIS and soma (Fig. 3.3Ba, arrow), but is present at the basket (b) AIS (Fig. 3.3Ba, b, arrowheads). In P12 and P16 wild type cerebella, Nfasc becomes enriched in the Purkinje soma and AIS (Fig. 3.3Da, b; Ga, b), as well as at the basket (b) AIS (Fig. 3.3Da, b, arrowheads). In P12 and P16 Pcp2-Cre;Nfasc ${ }^{\text {Flox }}$ cerebella, Nfasc is absent from the Purkinje soma and AIS (Fig. 3.3Ea, b; Ha, b; arrows), but is present at the basket (b) AIS (Fig. 3.3Ea, b; Ha, b; arrowheads). Interestingly, as the basket axon collaterals reach the Purkinje AIS and pinceau begins to take shape, Nfasc labels the basket axon collaterals at the pinceau area that is distinct from its localization at the Purkinje AIS (Fig. 3.3Da, b; Ga, b; Ja, b; Ma, b; $\mathrm{Pa}, \mathrm{b}$; green arrowheads). As the pinceau matures from P20, P30 and into adulthood (P150), Nfasc is not only localized to the Purkinje soma and AIS, but is also found at the pinceau, indicating that it is expressed in the basket axon terminals (Fig. 3.3Ja, b; Ma, b; $\mathrm{Pa}, \mathrm{b}$, green arrowheads). Most importantly, Nfasc localization in the basket axon terminals is not affected in $P c p 2-C r e ; N f a s c^{F l o x}$ cerebella at any of the developmental time points analyzed (Fig. 3.3Ka, b; Na, b; Qa, b; green arrowheads). Together, these data show that Nfasc is expressed in both Purkinje and basket neurons, and that in addition to its localization at the Purkinje soma/AIS, Nfasc also localizes at the basket AIS and basket axon terminals. 
To verify that Nfasc is expressed in basket neurons and localizes to basket axon terminals at the pinceau, we utilized a Parvalbumin-Cre (Parv-Cre) mouse strain (Hippenmeyer et al., 2005) that expresses Cre in all Parv-expressing interneurons in the nervous system and generated Parv-Cre; Nfasc ${ }^{\text {Flox }}$ mice. Similar to Pcp2-Cre; Nfasc ${ }^{\text {Flox }}$ mice, we confirmed loss of Nfasc in Purkinje and basket neurons at P0 (Fig. 3.2E). We carried out immunostaining for Nfasc to confirm ablation of Nfasc in Parv-Cre; Nfasc $^{\text {Flox }}$ mice (Fig. 3.3C, F, I, L, O, R). Analysis of P10 (Fig. 3.3Ca, b), P12 (Fig. 3.3Fa, b), P16 (Fig. 3.3Ia, b), P20 (Fig. 3.3La, b), P30 (Fig. 3.3Oa, b) and P150 (Fig. 3.3Ra, b) ParvCre;Nfasc ${ }^{\text {Flox }}$ cerebella showed loss of Nfasc in both Purkinje and basket neurons. Most importantly, Nfasc-positive immunostaining observed in wild type and Pcp2Cre;Nfasc ${ }^{\text {Flox }}$ cerebella was absent from the basket axon terminals surrounding the Purkinje AIS (Fig. 3.3Ib, Lb, Ob and Rb, green arrowheads). Higher magnification images of the Purkinje AIS/pinceau area from wild type (Fig. 3.3M, P), Pcp2Cre;Nfasc $^{\text {Flox }}$ (Fig. 3.3N, Q) and Parv-Cre;Nfasc ${ }^{\text {Flox }}$ (Fig. 3.3L, O) cerebella show that Nfasc is absent from Purkinje neurons in both Pcp2-Cre;Nfasc ${ }^{\text {Flox }}$ (Fig. 3.3U, V, asterisks) and Parv-Cre; Nfasc $^{\text {Flox }}$ (Fig. 3.3W, X; asterisks) cerebella. They also show that Nfasc is present in basket axon terminals surrounding the Purkinje AIS in wild type (Fig. 3.3S, T, green arrowheads) and that this staining is present in Pcp2-Cre;Nfasc ${ }^{\text {Flox }}$ (Fig. 3.3U, V) and absent in Parv-Cre; Nfasc $^{\text {Flox }}$ (Fig. 3.3W, X; green arrowheads) cerebella. These data demonstrate that Nfasc is expressed in both Purkinje and basket neurons, and that Nfasc localizes to basket axon collaterals, which form the pinceau at the Purkinje AIS. 


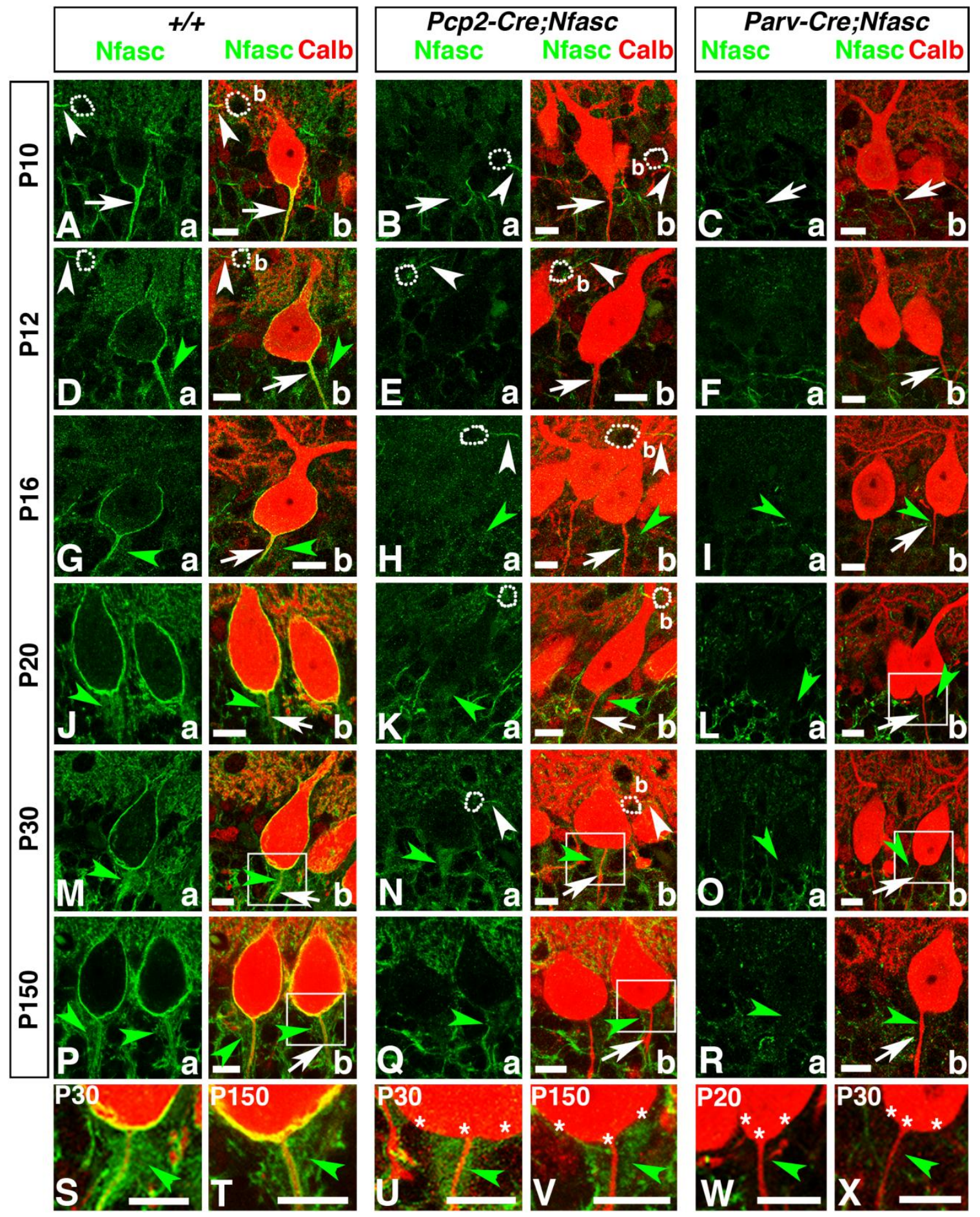

Figure 3.3 Purkinje and basket-specific ablation of Neurofascin reveals its localization to both Purkinje and basket neurons.

Wild type, Pcp2-Cre;Nfasc ${ }^{\text {Flox }}$ and Parv-Cre;Nfasc ${ }^{\text {Flox }}$ cerebellar sections from ages P10$\mathrm{P} 150$ as indicated on left (A-R) or within panel (S-X), and immunostained against Nfasc (a, green) and Calb (b, red). Note that in P10 wild type cerebella, Nfasc is localized to the Purkinje AIS (A, arrows) and basket (b) AIS (A, white arrowheads). At later stages, in 
wild type cerebella, Nfasc is localized around the entire Purkinje soma and AIS (D, G, J, $\mathrm{M}, \mathrm{P}$, arrows), as well as the basket AIS (D, white arrowhead). Nfasc is also localized to basket axon terminals as they form the pinceau in wild type cerebella (D, G, J, M, P, green arrowheads). In Pcp2-Cre; ffasc $^{\text {Flox }}$ cerebella, Nfasc is ablated from the Purkinje soma/AIS (B, E, H, K, N, Q, arrows), but remains at the basket AIS (B, E, H, N, white arrowhead) and pinceau (H, K, N, Q, green, arrowheads). In Parv-Cre;Nfasc ${ }^{\text {Flox }}$ cerebella, Nfasc is lost from the Purkinje soma/AIS (C, F, I, L, O, R, arrows), basket AIS and pinceau (I, L, O, R, green arrowheads). Higher magnification shows that Nfasc is present at the Purkinje soma/AIS and pinceau in wild type ( $\mathrm{S}, \mathrm{T}$, green arrowheads), is missing from the Purkinje soma/AIS in Pcp2-Cre; ffasc $^{F l o x}$ (U, V, stars), while remaining at the pinceau in Pcp2-Cre;Nfasc ${ }^{F l o x}$ (U, V, green arrowheads), and is missing from both the Purkinje soma/AIS (stars) and the pinceau (green arrowheads) in Parv-Cre;Nfasc Flox cerebella (W, X). Scale bars: $10 \mu \mathrm{m}$ 


\subsubsection{Nfasc is Required for the Maturation and Maintenance of the Purkinje Axon Initial Segment}

Studies examining the organization of the AIS in vivo and in vitro have led to a debate as to which proteins are critical in the initial organization of the AIS (Grubb and Burrone, 2010b). Loss of Nfasc globally does not affect initial clustering of AIS components AnkG, $\mathrm{Na}_{\mathrm{V}}$ channels and $\beta \mathrm{IV}$-Spectrin, but severely disrupts NrCAM localization at the AIS (Zonta et al., 2011). However, loss of Nfasc later in the adult life progressively leads to AIS disorganization (Zonta et al., 2011). RNAi knockdown of AnkG in in vitro hippocampal neuron cultures led to disorganization of the AIS and change in neuronal polarity, but AIS organization was relatively unaffected when Nfasc was knocked down (Hedstrom et al., 2008). These observations have suggested that initial AIS organization is dependent on AnkG, but independent of Nfasc, and that the maintenance of the AIS requires Nfasc. We wanted to address this question using the Pcp2-Cre;Nfasc ${ }^{\text {Flox }}$ and Parv-Cre;Nfasc ${ }^{\text {Flox }}$ mice to determine the consequences of loss of Nfasc in Purkinje neurons and how that affected the developmental organization of the AIS. At P10 in the wild type Purkinje neurons, AnkG localizes at the AIS and its expression extends distally from the AIS (Fig. 3.4Aa, b; arrowheads). This initial distribution of AnkG at the AIS is unaffected when Nfasc is lost in Pcp2-Cre;Nfasc ${ }^{\text {Flox }}$ and Parv-Cre;Nfasc ${ }^{\text {Flox }}$ mice (Fig. 3.4Ba, b; Ca, b, arrowheads). By P20, AnkG gets highly enriched at the AIS in the wild type (Fig. 3.4Da, b; arrowheads). In P20 Pcp2Cre;Nfasc ${ }^{\text {Flox }}$ and Parv-Cre;Nfasc ${ }^{\text {Flox }}$ cerebella AnkG fails to localize at the AIS and becomes mislocalized to the distal region of the AIS (Fig. 3.4Ea, b; Fa, b; arrowheads). By P30, in wild type cerebella, AnkG is concentrated at the AIS (Fig. 3.4Ga, b; 
arrowheads), whereas no AnkG is detectable in Pcp2-Cre; fasc $^{\text {Flox }}$ and ParvCre;Nfasc ${ }^{\text {Flox }}$ mutant AIS (Fig. 3.4Ha, b; Ia, b; arrows). These data suggest that Nfasc is not essential for initial localization of AnkG at the AIS, but is for its proper retention and stabilization at the AIS.

We next determined the consequences of loss of Nfasc on the developmental localization of $\mathrm{Na}_{\mathrm{V}}$ channels at the Purkinje AIS. It has been previously reported that the Purkinje AIS initially contains only Nav1.1 and later Nav1.6 becomes enriched at the AIS (Van Wart and Matthews, 2006). We carried out immunostaining using panNav and Nav1.6 specific antibodies. At P10, panNav staining showed proper clustering of $\mathrm{Na}_{\mathrm{v}}$ channels at the Purkinje AIS in Pcp2-Cre; ffasc $^{\text {Flox }}$ and Parv-Cre; ffasc $^{\text {Flox }}$ mutants (Fig. 3.4Ka, b; La, b; arrowheads), which was indistinguishable from that observed in the wild type (Fig. 3.4Ja, b; arrowheads). Interestingly, immunostaining against $\mathrm{Na}_{\mathrm{v}} 1.6$ at P10 showed localization of $\mathrm{Na}_{\mathrm{v}} 1.6$ distal to the AIS in the wild type cerebella (Fig. 3.4Ma, b; arrowheads; arrows point to AIS) and similar localization was observed in Pcp2Cre;Nfasc ${ }^{\text {Flox }}$ and Parv-Cre;Nfasc ${ }^{\text {Flox }}$ mutants (Fig. 3.4Na, b; Oa, b; arrowheads). These data indicate that $\mathrm{Na}_{\mathrm{v}} 1.6$ clustering initially does not occur at the AIS, but in the immediate distal region of the AIS. By P20, panNav immunostaining showed localization of $\mathrm{Na}_{\mathrm{V}}$ channels at the AIS in the wild type cerebella (Fig. 3.4Pa, b; arrowheads), but in Pcp2-Cre; fasc $^{\text {Flox }}$ and Parv-Cre; Nfasc $^{\text {Flox }}$ mutants, $\mathrm{Na}_{\mathrm{v}}$ channel localization was already severely affected with localization toward the distal regions from the AIS (Fig. 3.4Qa, b; Ra, b; arrowheads). Similarly, Nav1.6 immunostaining at P20 revealed that Nav1.6 becomes highly enriched at the wild type Purkinje AIS (Fig. 3.4Sa, b; arrowheads). In Pcp2-Cre;Nfasc ${ }^{\text {Flox }}$ and Parv-Cre;Nfasc ${ }^{\text {Flox }}$ mutants, by P20, Nav1.6 
remained mislocalized in the distal axonal region away from the AIS (Fig. 3.4Ta, b; Ua, b; arrowheads). At P30, both panNav and $\mathrm{Na}_{\mathrm{v}} 1.6$ are enriched at the wild type Purkinje AIS (Fig. 3.4Va, b; Ya,b; arrowheads), but are undetectable at the AIS as well as at the distal region of the AIS in P30 Pcp2-Cre;Nfasc ${ }^{\text {Flox }}$ and Parv-Cre; ffasc $^{\text {Flox }}$ mutants (Fig. 3.4Wa, b; Xa, b; Za, b; Z'a, b; arrows). Together, these data clearly show that the initial phase of AIS organization is independent of Nfasc, and that Nfasc is critically required for the second phase, i.e. the maturation of the AIS with the localization of Nav1.6, and the maintenance of the molecular components of the AIS. 


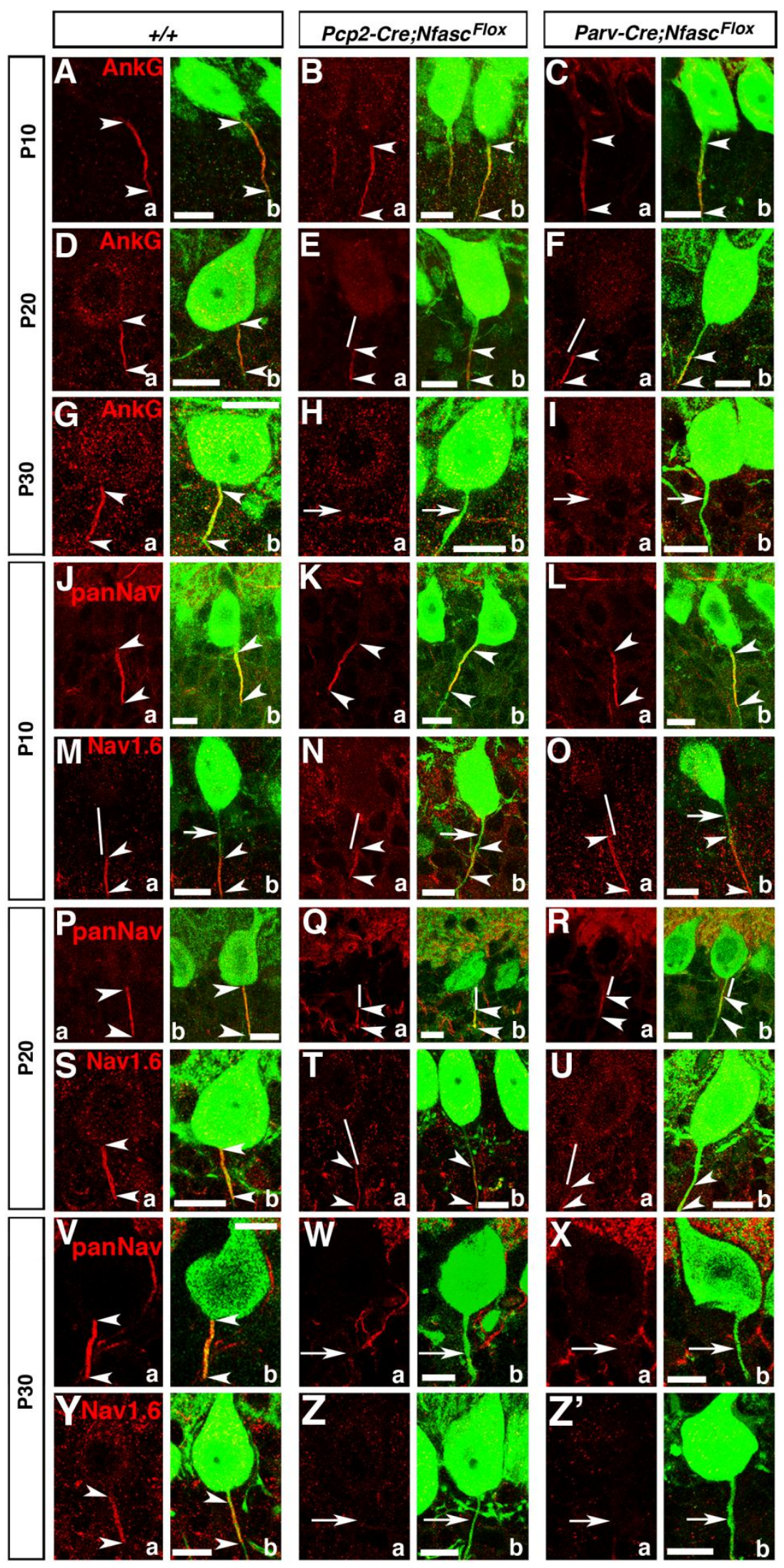


Figure 3.4 Early postnatal loss of Nfasc disrupts Purkinje AIS maturation and stabilization.

Wild type, Pcp2-Cre;Nfasc ${ }^{\text {Flox }}$ and Parv-Cre;Nfasc ${ }^{\text {Flox }}$ cerebellar sections from mice ages P10-30 immunostained against AnkG (A-I, a, red), panNav (J-L; P-R, V-X, a, red), Nav1.6 (M-O; S-U, Y-Z', a, red) and Calb (A-Z', b, green). AnkG is localized (between arrowheads) to the wild type Purkinje AIS at P10, P20 and P30. In both mutants, AnkG remains (between arrowheads) at the Purkinje AIS at P10, moves distally down the axon at P20 (E, F, between arrowheads) and is undetectable from the Purkinje axon by P30 (H, I, arrows). At P10 (J, between arrowheads), P20 (P, between arrowheads), and P30 (V, between arrowheads) panNav shows AIS localization in wild type Purkinje AIS.

Localization of panNa $\mathrm{V}_{\mathrm{V}}$ remains the same as in the wild type in both mutants at P10 (K, $\mathrm{L}$, between arrowheads), but at P20 panNav shows severely reduced immunostaining at the mutant AISs (Q, R, between arrowheads), and is completely missing from the P30 mutant AISs (W, X, arrows). Localization of Nav1.6 at the wild type AIS at P10 shows that Nav1.6 is located distally in the AIS (M, between arrowheads) and moves to fill the entire AIS at P20 and P30 (S, Y, between arrowheads). Localization of $\mathrm{Na}_{\mathrm{V}} 1.6$ remains the same as wild type in both mutants at P10 (N, O, between arrowheads) and remains distally localized at P20 (T, U, between arrowheads), and is undetectable at the AIS at P30 (Z, Z', arrows). The white line indicates the location of AIS in E, F, M-O, Q, R, T and U). Scale bars: $10 \mu \mathrm{m}$ 


\subsubsection{Nfasc is Required in both Basket Neurons and Purkinje Neurons for the Organization of the Pinceau}

A hallmark of the Purkinje neuron AIS is that the basket neuron collaterals establish a unique structure, the pinceau, at the AIS (refer Fig. 3.1). Our finding that, in addition to Nfasc localization at the Purkinje AIS, Nfasc is also expressed in the basket axon collaterals has raised an interesting question whether Nfasc is bidirectionally required for proper pinceau organization. Since Pcp2-Cre caused loss of Nfasc in Purkinje neurons and Parv-Cre resulted in the loss of Nfasc in both Purkinje and basket neurons, we compared the development of the pinceau and the phenotypes thereof between Pcp2-Cre;Nfasc ${ }^{\text {Flox }}$ and Parv-Cre;Nfasc ${ }^{\text {Flox }}$ mutants. We carried out triple immunostaining against pNfl, $\mathrm{K}_{\mathrm{V}} 1.2$ and Calb (refer Fig. 3.1) to determine the extent of pinceau disorganization in these mutants (Fig. 3.5). As noted earlier (Fig. 3.1) at P10 and P12, very few basket axon collaterals have reached the Purkinje AIS by these developmental stages and no significant differences were observed at the Purkinje AISs between wild type, Pcp2-Cre;Nfasc ${ }^{\text {Flox }}$ and Parv-Cre;Nfasc ${ }^{\text {Flox }}$ mutants (Fig. 3.5Ba-c; Ca-c; compare with Fig. 3.5Aa-c; Ea-c; Fa-c; compare with Fig. 3.5Da-c). At P16, in wild type cerebella many basket axon collaterals reach the Purkinje AIS (Fig. 3.1Ga, c) and $\mathrm{K}_{\mathrm{V}} 1.2$ channels become enriched at the pinceau (Fig. 3.1Gb, c, arrowhead). In P16 Pcp2-Cre; fasc $^{\text {Flox }}$ cerebella, $\mathrm{K}_{\mathrm{V}} 1.2$ channels get enriched at the basket axon terminals that have reached the Purkinje AIS, but fail to get properly organized to form a compact structure compared to the wild type (Fig. $3.5 \mathrm{Hb}$, c; arrowheads). In P16 Parv-

Cre;Nfasc ${ }^{\text {Flox }}$ cerebella, $\mathrm{K}_{\mathrm{V}} 1.2$ channels fail to get enriched at the Purkinje AIS and instead show small ectopic clusters near the Purkinje soma and regions distal to Purkinje 
AIS (Fig. 3.5Ib, c; arrowheads). Quantification of the percent of non-compact pinceau, based on $\mathrm{K}_{\mathrm{V}} 1.2$ localization, revealed that at P16,33.3\% of wild type pinceau were not compact ( $\mathrm{n}=48$ Purkinje cells from 4 separate animals), while this number increased to $82.5 \%$ in Pcp2-Cre;Nfasc ${ }^{\text {Flox }}$ cerebella ( $\mathrm{n}=42$ Purkinje cells from 4 separate animals) and 76.2\% in Parv-Cre; Nfasc $^{\text {Flox }}$ cerebella (n=40 Purkinje cells from 4 separate animals). In addition, quantification of ectopic $\mathrm{K}_{\mathrm{V}} 1.2$ clustering around the Purkinje soma at P16 revealed that $52.4 \%$ of Purkinje neurons in Parv-Cre;Nfasc ${ }^{\text {Flox }}$ cerebella had ectopic clusters, compared to $35.0 \%$ in Pcp2-Cre; Ffasc $^{\text {Flox }}$ and $8.3 \%$ in wild type cerebella at this age. In P20 Pcp2-Cre;Nfasc ${ }^{\text {Flox }}$ cerebella, $\mathrm{K}_{\mathrm{v}} 1.2$ channels get enriched at the basket axon terminals that have reached the Purkinje AIS area, but begin to form small unstructured $\mathrm{K}_{\mathrm{V}} 1.2$ clusters (Fig. 3.5Kb, c; arrowheads; compare with wild type Fig. 3.5Jb, c; arrowheads). In P20 Parv-Cre; Nfasc ${ }^{\text {Flox }}$ cerebella, $\mathrm{K}_{\mathrm{V}} 1.2$ channels either form tiny clusters or small stretched clusters near the Purkinje soma and regions distal to Purkinje AIS (Fig. 3.5Lb, c; arrowheads), or completely fail to form any $\mathrm{K}_{\mathrm{V}} 1.2$ clusters at the Purkinje AIS (data not shown). At P20, quantification of the percent of non-compact $\mathrm{K}_{\mathrm{V}} 1.2$ pinceau showed that $41.7 \%$ of pinceau were disorganized in Pcp2-Cre; ffasc $^{\text {Flox }}$ cerebella ( $n=36$ Purkinje cells from 4 separate animals) and 56.8\% were disorganized in Parv-Cre; Nasc $^{\text {Flox }}$ cerebella ( $\mathrm{n}=44$ Purkinje cells from 4 separate animals), compared to $14.0 \%$ in wild type cerebella ( $\mathrm{n}=43$ Purkinje cells from 4 separate animals). At P20, there were also ectopic $\mathrm{K}_{\mathrm{V}} 1.2$ clusters around $59.0 \%$ of Parv-Cre;Nfasc ${ }^{\text {Flox }}$ Purkinje neurons, $30.0 \%$ of Pcp2-Cre;Nfasc ${ }^{\text {Flox }}$ Purkinje neurons, and $18.6 \%$ of wild type Purkinje neurons. By P30, Pcp2-Cre; $N f a s c^{\text {Flox }}$ cerebella show basket axon collaterals that are mistargeted to the distal areas of the Purkinje AIS and form broken clusters of $\mathrm{K}_{\mathrm{V}} 1.2$ channels at the 
Purkinje AIS area (Fig. 3.5Nb, c; arrowheads; compare with wild type Fig. 3.5Mb, c; arrowhead). By P30, Parv-Cre;Nfasc ${ }^{\text {Flox }}$ cerebella show a total failure of $\mathrm{K}_{\mathrm{V}} 1.2$ channel clustering at the pinceau (Fig. 3.5Ob, c; arrowheads; compare with wild type Fig. 3.5Mb, c; arrowhead). In P30 Parv-Cre;Nfasc ${ }^{\text {Flox }}$ cerebella, $73.3 \%$ pinceau were disorganized ( $\mathrm{n}=86$ Purkinje cells from 4 separate animals), based on $\mathrm{K}_{\mathrm{V}} 1.2$ localization, compared to $70.9 \%$ in Pcp2-Cre;Nfasc ${ }^{\text {Flox }}$ cerebella (n=55 Purkinje cells from 4 separate animals) and $19.5 \%$ in wild type cerebella ( $\mathrm{n}=77$ Purkinje cells from 4 separate animals). Interestingly, ectopic clusters of $\mathrm{K}_{\mathrm{V}} 1.2$ around the Purkinje soma were not as high in P30 mutant cerebella, compared to $\mathrm{P} 16$, with ectopic $\mathrm{K}_{\mathrm{V}} 1.2$ clusters surrounding $24.4 \%$ of ParvCre;Nfasc ${ }^{\text {Flox }}$ Purkinje neurons, $14.6 \%$ of Pcp2-Cre;Nfasc ${ }^{\text {Flox }}$ Purkinje neurons and $2.6 \%$ of wild type Purkinje neurons.

We also analyzed 5-month old wild type, Pcp2-Cre;Nfasc ${ }^{\text {Flox }}$ and ParvCre;Nfasc ${ }^{\text {Flox }}$ mutant cerebella to determine the extent of pinceau defects at the Purkinje AIS. While the wild type Purkinje AIS showed proper targeting of basket axon collaterals and cone-shaped pinceau (Fig. 3.5Pa, b; arrowhead), Pcp2-Cre;Nfasc ${ }^{\text {Flox }}$ (Fig. 3.5Qa, b; arrowheads) and Parv-Cre;Nfasc ${ }^{\text {Flox }}$ (Fig. 3.5Ra, b; arrowheads) mutant cerebella revealed severely disorganized pinceau. In Parv-Cre;Nfasc ${ }^{\text {Flox }}$ mutants, PSD95 clustering at the pinceau was either completely absent or formed small patchy clusters at ectopic locations at the Purkinje soma or somewhere around the Purkinje AIS. Quantification analyses of the basket axon collaterals that targeted the Purkinje AIS and soma area in P20 Pcp2-Cre;Nfasc ${ }^{\text {Flox }}$ and Parv-Cre;Nfasc ${ }^{\text {Flox }}$ mutant cerebella are shown in Fig. 3.5S. This revealed that fewer basket axon collaterals surround the Purkinje AIS in both mutants, compared to wild type (WT: 14.42 axons per AIS, n=31 Purkinje cells from 3 
separate animals; Pcp2-Cre;Nfasc ${ }^{\text {Flox }}$ : 9.64 axons per AIS, $\mathrm{n}=22$ Purkinje cells from 3 separate animals, $\mathrm{p}=0.00008$; Parv-Cre;Nfasc ${ }^{\text {Flox }}$ : 10.35 axons per AIS, n=34 Purkinje cells from 3 separate animals, $\mathrm{p}=0.00014)$. However, no significant differences were found in the number of collaterals surrounding the soma in each genotype (WT: 17.55 axons per soma, $\mathrm{n}=31$ Purkinje cells from 3 separate animals; Pcp2-Cre;Nfasc ${ }^{\text {Flox }}: 17.09$ axons per soma, $\mathrm{n}=22$ Purkinje cells from 3 separate animals, $\mathrm{p}=0.73$; ParvCre; Nfasc ${ }^{\text {Flox }}: 20.35$ axons per AIS, $\mathrm{n}=34$ Purkinje cells from 3 separate animals, $\mathrm{p}=0.11$ ). A one-way ANOVA was also used to test for statistical difference in the mean number of basket axon terminals at the Purkinje AIS and at the Purkinje soma between the wild-type, Pcp2-Cre;Nfasc ${ }^{\text {Flox }}$, and Parv-Cre;Nfasc ${ }^{\text {Flox }}$ cerebella. The number of basket axon terminals at the Purkinje AIS differed significantly across the three genotypes, $\mathrm{F}(2,84)=13.05, \mathrm{p}=0.000012$. However, the number of basket axon terminals at the Purkinje soma did not differ significantly across the three genotypes, $F(2,84)=2.27$, $\mathrm{p}=0.109$. These data show that loss of Nfasc from either the Purkinje neuron or Purkinje and basket neurons results in decreased basket axon targeting to the Purkinje AIS. However, it is not clear from these results whether decreased collaterals are due to destabilization defects or targeting defects. 


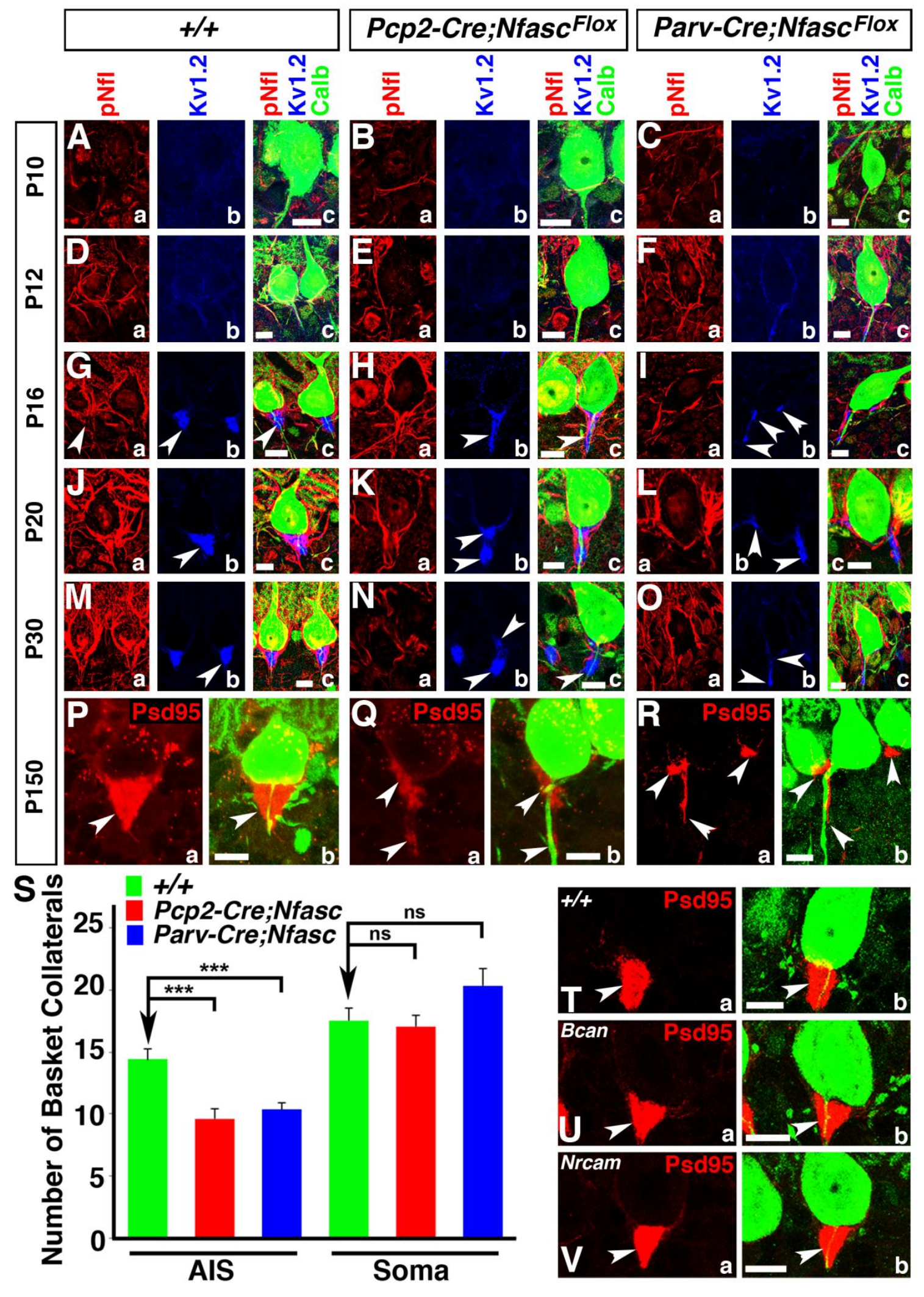


Figure 3.5 Ablation of Nfasc disrupts basket axon targeting and pinceau organization.

Wild type (A, D, G, J, M), Pcp2-Cre; ffasc $^{\text {Flox }}$ (B, E, H, K, N) and Parv-Cre;Nfasc ${ }^{\text {Flox }}$ (C, F, I, L, O) cerebellar sections from ages P10 (A-C), P12 (D-F), P16 (G-I), P20 (J-L) and P30 (M-O) immunostained against pNfl (a, red), $\mathrm{K}_{\mathrm{V}} 1.2$ (b, blue), and Calb (c, green, merged). Pinceau disruption becomes evident in Pcp2-Cre; fasc $^{\text {Flox }}$ cerebella at P16, when fewer collaterals appear to reach the Purkinje AIS (Ha, c) and $\mathrm{K}_{\mathrm{V}} 1.2$ does not become as enriched as at the wild type pinceau ( $\mathrm{Hb}, \mathrm{c})$. This trend continues at P20 and P30, where fewer collaterals reach the Purkinje AIS (Ka, Na) and potassium channels fail to cluster properly and appear as broken clusters compared to wild type (Kb, $\mathrm{Nb})$. In Parv-Cre; Nfasc $^{\text {Flox }}$ cerebella at P16, basket axon targeting is disrupted (Ia, c) and $\mathrm{K}_{\mathrm{V}} 1.2$ clusters ectopically around the soma and at random places along the Purkinje AIS (Ib, c). This disruption continues at P20 and P30 in Parv-Cre;Nfasc ${ }^{\text {Flox }}$ cerebella with altered basket axon targeting ( $\mathrm{La}, \mathrm{Oa}$ ) and ectopic $\mathrm{K}_{\mathrm{V}} 1.2$ clusters $(\mathrm{Lb}, \mathrm{Ob})$. Wild type $(\mathrm{P}, \mathrm{T})$,

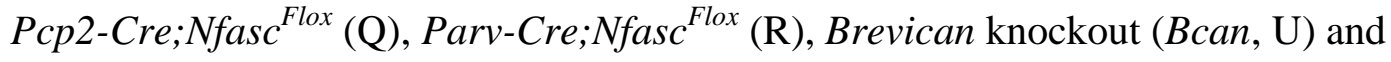
$\mathrm{NrCAM}$ knockout (V) cerebella immunostained against PSD95 (a, red) and Calb (b, green, merged). At P150, the wild type pinceau maintains its cone-shaped PSD95 localization $(\mathrm{P}, \mathrm{T})$, while it is completely disrupted in both Pcp2-Cre;Nfasc ${ }^{F l o x}(\mathrm{Q})$ and Parv-Cre; Nfasc ${ }^{\text {Flox }}$ cerebella (R). (S) Quantification of basket axon collateral targeting to the Purkinje AIS and soma in Pcp2-Cre;Nfasc ${ }^{\text {Flox }}$ and Parv-Cre;Nfasc ${ }^{\text {Flox }}$ cerebella. The pinceau remains intact in both $B c a n(\mathrm{U})$ and $\operatorname{NrCAM}(\mathrm{V})$ mutants. Scale bars: $10 \mu \mathrm{m}$. ns-not significant. $* * *_{-}-\mathrm{p}<0.001$. 
Next we determined whether the pinceau disorganization defects observed in Nfasc mutants are also seen in Brevican (Bcan) and Nrcam mutants. Brevican encodes a proteoglycan which localizes to Purkinje AIS and has been shown to interact with $\mathrm{Nfasc}^{\mathrm{NF186}}$ (Hedstrom et al., 2007). Interestingly, Brevican localization was not altered in either Pcp2-Cre;Nfasc ${ }^{\text {Flox }}$ or Parv-Cre;Nfasc ${ }^{\text {Flox }}$ cerebella (Fig. 3.6D-E, compare to Fig. 3.6C). Nrcam encodes a neuron glia-cell adhesion molecule ( $\mathrm{NrCAM})$ that is enriched at the Purkinje AIS and gets mislocalized in Nfasc mutants (Zonta et al., 2011). Both Brevican (Bcan) (Fig. 3.5Ua, b; Fig. 3.6Ga, b; Fig. 3.6Ja, b; arrowhead) and Nrcam (Fig. 3.5Va, b; Fig. 3.6Ha, b; Fig. 3.6Ka, b; arrowhead) null mutants revealed normal organization of Nfasc and the pinceau, as seen in the wild type cerebellum (Fig. 3.5Ta, b; Fig. 3.6Fa, b; Fig. 3.6I a, b; arrowhead), indicating that Brevican and $\mathrm{NrCAM}$ are not critically required for the localization of Nfasc or for the organization of the cerebellar pinceau. Together, our data suggest that loss of Nfasc in both basket and Purkinje neurons leads to more severe disorganization of the cerebellar pinceau than when Nfasc is lost only in the Purkinje neurons. 

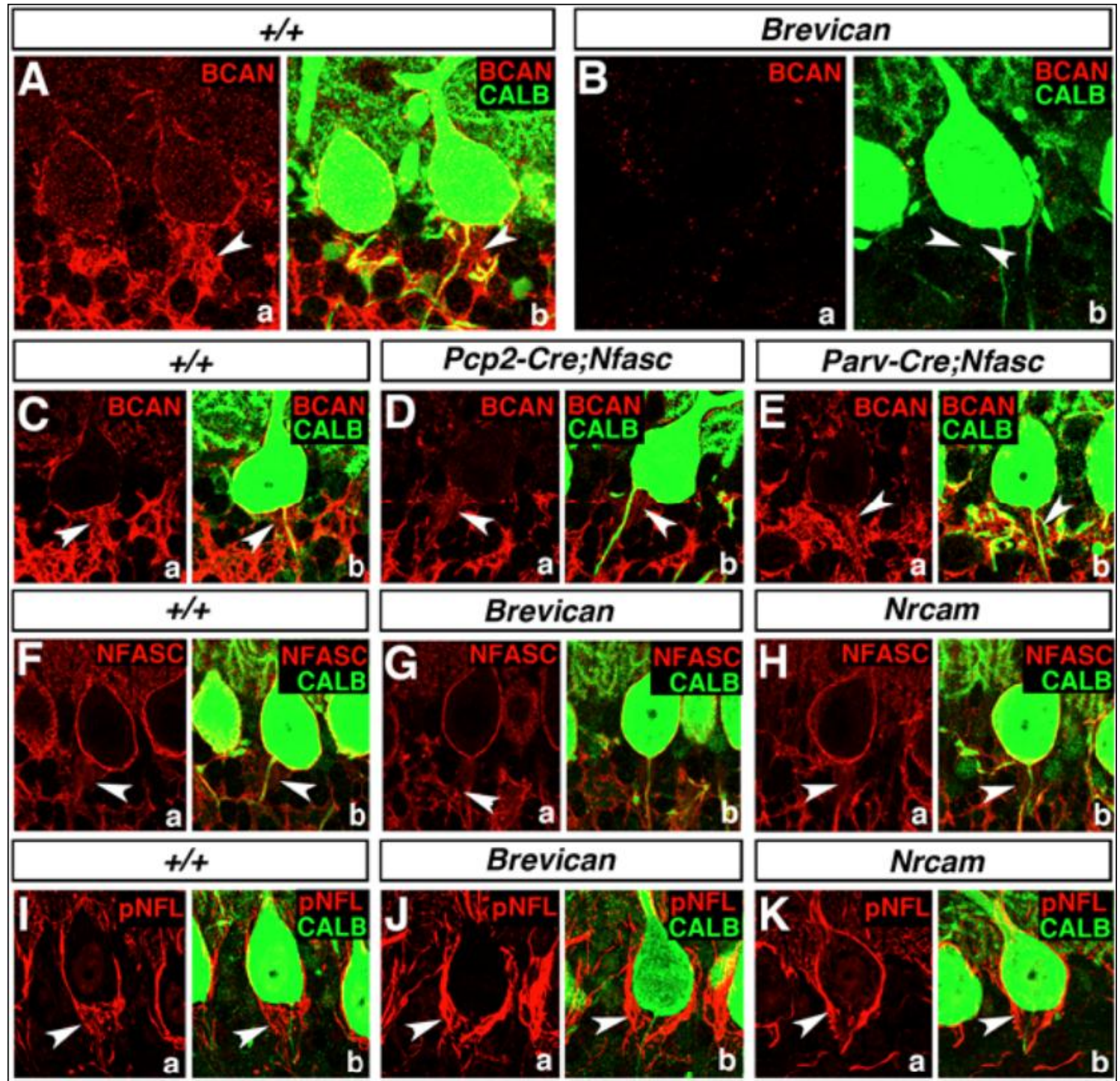

Figure 3.6 Pinceau formation is not affected in Brevican and $\mathbf{N r C A M}$ mutants. Wild type (A, C, F, I), Brevican-/- (Bcan, B, G, J), Pcp2-Cre;Nfasc ${ }^{\text {Flox }}$ (D), ParvCre;Nfasc ${ }^{\text {Flox }}$ (E), and NrCAM-/- (Nrcam, H, K) cerebellar sections immunostained against Brevican (Aa - Ea, red), Nfasc ( $\mathrm{Fa}-\mathrm{Ha}$, red), pNfl (Ia - Ka, red), and Calb (b, green, merged). Brevican is localized around the Purkinje soma and AIS in wild type (Aa, Ca) and is relatively unaffected around the Purkinje soma and AIS in Pcp2Cre; Nfasc $^{\text {Flox }}(\mathrm{Da})$ and Parv-Cre; Nfasc $^{\text {Flox }}$ (Ea) cerebella. Wild type Nfasc localization at the Purkinje soma and AIS (Fa) is maintained in both Brevican $(\mathrm{Ga})$ and $\operatorname{Nrcam}(\mathrm{Ha})$ mutant cerebella. Basket axon collateral targeting is also unaffected in Brevican (Ja) and Nrcam (Ka) mutant cerebella, as revealed by pNfl immunostaining. 


\subsubsection{Nfasc is Required in Basket Neurons for Proper Axon Collateral Outgrowth and Targeting during Pinceau Formation.}

Our immunohistochemical analyses indicated that loss of Nfasc in Purkinje and basket neurons led to a decrease in the number of basket axon collaterals that targeted to the Purkinje AIS, and that pinceau formation was severely compromised. This phenotype could be caused by defects in basket axon collateral guidance and/or outgrowth during cerebellar development, or due to inability of basket collaterals to form stable contacts at the Purkinje AIS. Earlier studies using chick have suggested that Nfasc interacts homophilically to induce neurite outgrowth (Pruss et al., 2004) and that alternative splicing of Nfasc allows for expression of multiple Nfasc isoforms, which either promote or inhibit neurite extension and cell adhesion (Koticha et al., 2005; Pruss et al., 2006). However, the in vivo role of Nfasc in neurite outgrowth and adhesion has not been examined. Since Nfasc is localized to the basket axon terminals and loss of Nfasc in Purkinje (Pcp2-Cre;Nfasc ${ }^{\text {Flox }}$ ) or Purkinje and basket neurons (Parv-Cre;Nfasc ${ }^{\text {Flox }}$ ) caused different pinceau phenotypes, we hypothesized that Nfasc may play a unique role in basket axon terminals for pinceau formation. To address whether loss of Nfasc in basket neurons had any consequences on the basket axon collateral outgrowth and/or stabilization of basket axon collaterals, we performed single-cell fills of individual basket cells with biocytin in wild type, Pcp2-Cre;Nfasc ${ }^{\text {Flox }}$ and Parv-Cre;Nfasc ${ }^{\text {Flox }}$ mutants to follow basket axon collateral targeting. Biocytin filled basket neurons were then immunostained to detect biocytin (refer Experimental Procedures for details). At P10, as previously observed by pNfl immunostaining (refer Fig. 3.1), few basket axon collaterals reach the Purkinje neuron layer and begin to extend toward the Purkinje soma and AIS 
(Fig. 3.7A, arrow). In P10 Pcp2-Cre; Nfasc ${ }^{\text {Flox }}$ cerebella, the main basket axon extends through the molecular layer towards the Purkinje cell layer (Fig. 3.7B, arrow), but branches extensively into collaterals (Fig. 3.7B, arrowheads), indicating that Purkinje soma/AIS-localized Nfasc is required for terminating basket axon extension and forming stable contacts at the Purkinje AIS. In P10 Parv-Cre;Nfasc ${ }^{\text {Flox }}$ cerebella, the basket axons branched excessively, and failed to extend normally around the Purkinje soma (Fig. 3.7C, arrowheads), indicating that basket axon/collateral outgrowth fails to occur properly in the absence of Nfasc in basket cells. As development continues, wild type basket axons extend more branches towards the Purkinje cell layer (Fig. 3.7D; arrowheads). Similar to the phenotype seen in P10 Pcp2-Cre;Nfasc ${ }^{\text {Flox }}$ cerebella, at P20, the basket axons appear to target correctly to the Purkinje cell layer areas, but branch excessively once they arrive at the Purkinje cell layer (Fig. 3.7E, G; arrowheads), indicating that Nfasc at the Purkinje soma/AIS may be needed to terminate collateral extensions and to form stable contacts at the AIS. Remarkably, in P20 Parv-Cre;NFasc ${ }^{\text {Flox }}$ cerebella, the basket axons appear completely lost, branching in multiple directions and seeming to circle around the molecular layer, with no clear targeting pattern (Fig. 3.7F, H; arrowheads). In many instances, the collaterals fail to target towards the Purkinje soma/AIS (Fig. 3.7H; asterisks). A quantitative analysis of the basket axon collateral outgrowth and targeting revealed that loss of Nfasc in basket neurons causes more primary axon collateral branches than when Nfasc is only absent in Purkinje neurons (Fig. 3.7I). (P10 WT: 9.55 branches per axon, $\mathrm{n}=11$ basket neurons from 2 separate animals; P10 Pcp2Cre;Nfasc ${ }^{\text {Flox }}: 10.17$ branches per axon, $\mathrm{n}=6$ basket neurons from 2 separate animals, p=0.77; P10 Parv-Cre; Nfasc $^{\text {Flox }}$ : 16.71 branches per axon, $\mathrm{n}=7$ basket neurons from 2 
separate animals, $\mathrm{p}=0.036 ; \mathrm{P} 20 \mathrm{WT}$ : 12.5 branches per axon, $\mathrm{n}=6$ basket neurons from 2 separate animals; P20 Pcp2-Cre;Nfasc ${ }^{\text {Flox }}: 13.91$ branches per axon, n=11 basket neurons from 2 separate animals, $\mathrm{p}=0.42$; $\mathrm{P} 20$ Parv-Cre; Nfasc $^{\text {Flox }}$ : 20.13 branches per axon, $\mathrm{n}=8$ basket neurons from 2 separate animals, $\mathrm{p}=0.0014)$. A one-way ANOVA was used to test for statistical difference in the mean number of branches off the primary basket axon in both P10 and P20 cerebella from the three genotypes. The number of branches coming off the basket axon differed significantly at both $\mathrm{P} 10, \mathrm{~F}(2,21)=4.86, \mathrm{p}=0.018$, and at $\mathrm{P} 20$, $\mathrm{F}(2,22)=7.61, \mathrm{p}=0.003$. A schematic of the pinceau organization in wild type, $P c p 2$ Cre; Nfasc $^{\text {Flox }}$ and Parv-Cre;Nfasc ${ }^{\text {Flox }}$ mutant cerebella reflects the phenotypes that are observed when Nfasc is lost in Purkinje, and Purkinje and basket neurons (Fig. 3.7J). Together, our results suggest that Nfasc function is required in both Purkinje and basket neurons for proper axon collateral extension and targeting to the Purkinje AIS and for their stabilization at the AIS to allow for the formation and organization of the pinceau during cerebellar development. 

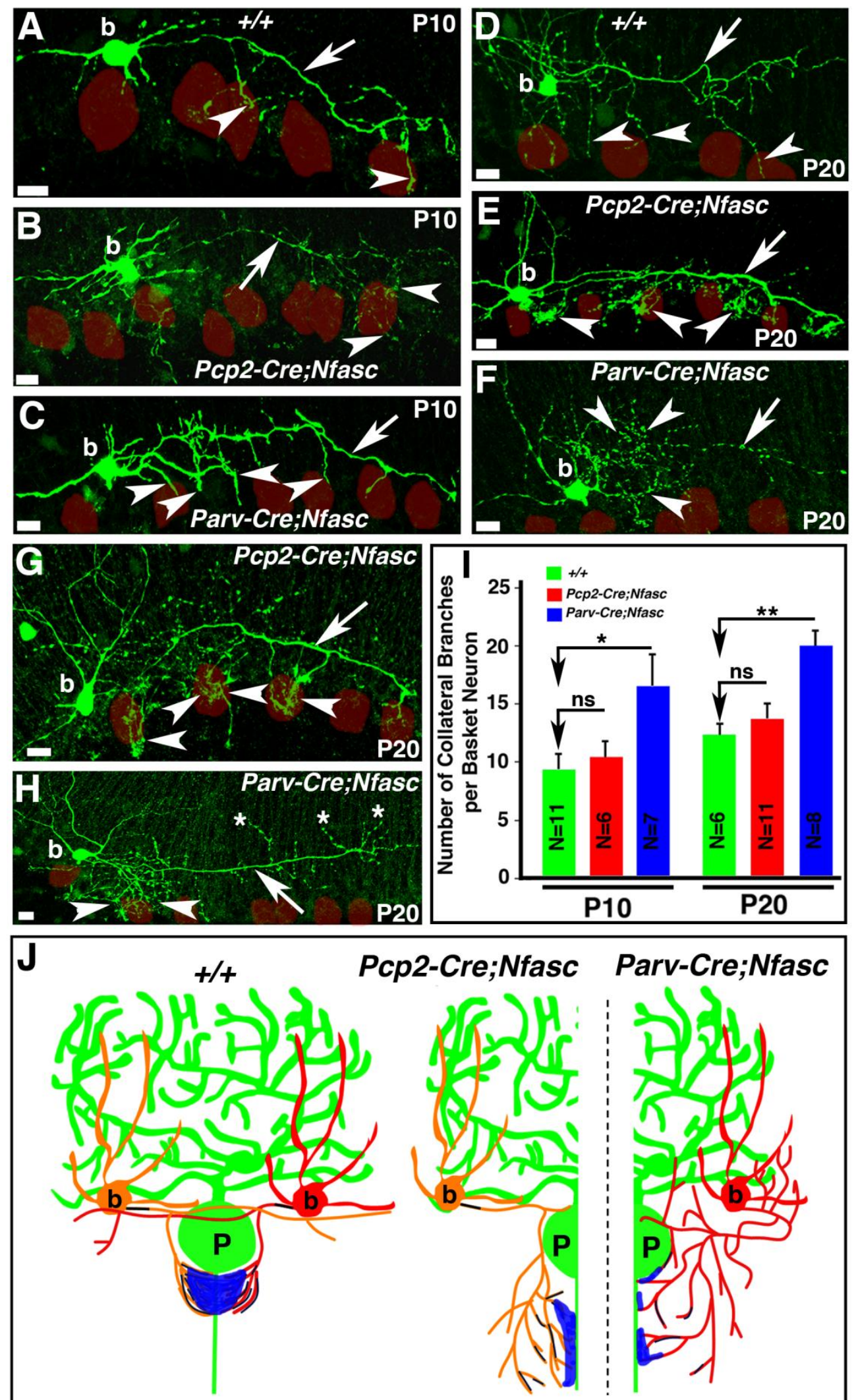
Figure 3.7 Loss of Nfasc in basket neurons causes mistargeting of basket axon collaterals.

Wild type (A, D), Pcp2-Cre;Nfasc ${ }^{\text {Flox }}$ (B, E, G), and Parv-Cre;Nfasc ${ }^{\text {Flox }}$ (C, F, H) cerebellar sections from ages P10 (A-C) and P20 (D-H) in which basket cells were injected with biocytin and immunostained with Alexa-conjugated Streptavidin (green). Note that basket axons project towards the Purkinje neuron layer in Pcp2-Cre;Nfasc ${ }^{\text {Flox }}$ mutants, but branch excessively at their terminals (B, E, G, arrowheads). In ParvCre; fasc $^{\text {Flox }}$ mutants, basket axons branch excessively from the beginning of the extension $(\mathrm{C}, \mathrm{F}, \mathrm{H}$, arrowheads) and sometimes point in opposite directions $(\mathrm{H}$, asterisks). Purkinje somas are outlined as red circles.

(I) Quantification of the number of primary basket terminal branches coming off the main basket axon branch at each time point reveal an increase in branching along the length of the Parv-Cre; fasc $^{\text {Flox }}$ basket axon compared to wild type and Pcp2-Cre; fasc $^{\text {Flox }}$ basket neurons.

(J) Representative drawings summarizing the pinceau structures observed in wild type, Pcp2-Cre; Nfasc ${ }^{\text {Flox }}$ and Parv-Cre; ffasc $^{\text {Flox }}$ cerebella. Scale bars: $10 \mu \mathrm{m}$. ns--not significant. $*_{--}$p $<0.05$. $*_{--}$p $<0.01$. 


\subsubsection{Nfasc is Required for Proper Basket Axon Collateral Targeting and Compaction at the Pinceau}

Several studies have proposed a role for Nfasc in GABAergic synapse formation at the AIS. Expression of a dominant-negative form of Nfasc in Purkinje cells disrupted pinceau synapse formation, based on GAD65 immunostaining (Ango et al., 2004). In addition, shRNA-knockdown of Nfasc in granular cells of the rat dentate gyrus resulted in a decrease of pre- and post-synaptic components (Kriebel et al., 2011). Although synaptic markers clearly define the cone-shape of the pinceau, only a few synapses are actually made by basket axons onto the Purkinje AIS at each pinceaux (Somogyi and Hamori, 1976; Sotelo, 2008). No ultrastructural analysis of the pinceau region has been carried out in AnkG mutants or animals that express transgenic dominant negative Nfasc (Ango et al., 2004) or in Nfasc adult mutants (Zonta et al., 2011). To determine the ultrastructural changes that occur at the pinceau as a result of loss of Nfasc in Purkinje, and Purkinje and basket neurons, we carried out EM analyses. EM analysis of one month wild type cerebella at the Purkinje AIS and the pinceau revealed a high density of basket (B) axon collaterals surrounding the Purkinje (P) soma and AIS and presence of synapses between basket collaterals and the Purkinje AIS (Fig. 3.8A, arrowheads). The Purkinje soma/AIS membrane is highlighted by the red line to distinguish it from the basket

collateral areas. In P30 Pcp2-Cre; ffasc $^{\text {Flox }}$ cerebella, we observed a decrease in the density of basket axons and clear gaps between the Purkinje soma/AIS and basket collaterals (Fig. 3.8B, arrows), but basket axons were able to form synapses with the Purkinje soma and AIS (Fig. 3.8B, arrowhead). In P30 Parv-Cre;Nfasc ${ }^{\text {Flox }}$ cerebella, we observed a further decrease of the basket collaterals at the pinceau and more gaps 
between the Purkinje soma/AIS and basket axon collaterals (Fig. 3.8C, arrows). Again, basket collaterals were able to form synapses with the Purkinje soma/AIS, indicating that loss of Nfasc in Purkinje and basket neurons does not abolish synapse formation (Fig. 3.8E, F, compare with wild type Fig. 3.8D). Quantification of the number of synapses formed on the Purkinje soma/AIS at one month revealed no significant differences in the total number of synapses between wild type, Pcp2-Cre;Nfasc ${ }^{\text {Flox }}$ and Parv-Cre;Nfasc ${ }^{\text {Flox }}$ mice (Wild type: 5.03 synapses per soma, $\mathrm{n}=105$ Purkinje neurons; Pcp2-Cre;Nfasc ${ }^{\text {Flox }}$ : 4.48 synapses per soma, n=75 Purkinje neurons; Parv-Cre;Nfasc ${ }^{\text {Flox }}: 4.13$ synapses per soma, $\mathrm{n}=68$ Purkinje neurons; $\mathrm{F}(2,245)=2.65, \mathrm{p}=0.073)$. These data suggest that basket axon collaterals retain their ability to form synapses with the Purkinje soma/AIS in the absence of Nfasc, indicating that Nfasc is not required for synapses formation. Next we analyzed 3 month old wild type cerebella, which showed that basket axon collaterals remain tightly compacted surrounding the Purkinje AIS area (Fig. 3.8G). However, at 3 months, both Pcp2-Cre; Nfasc $^{\text {Flox }}$ and Parv-Cre;Nfasc ${ }^{\text {Flox }}$ cerebella revealed more severe disorganization of the pinceau with glial processes, which may be from astrocytes undergoing gliosis, infiltrating the space between mistargeted basket axon collaterals (Fig. 3.8H, I; arrows). At 4 months, both mutants displayed more severe disorganization at the pinceau with increased infiltration of glial processes between mislocalized basket axon collaterals (Fig. 3.8K, L, N, O; arrows). Wild type cerebella at 4 months maintained a compact array of basket collaterals at the pinceau (Fig. 3.8J, M). Together, the ultrastructural analysis of Pcp2-Cre;Nfasc ${ }^{\text {Flox }}$ and Parv-Cre;Nfasc ${ }^{\text {Flox }}$ cerebella shows that loss of Nfasc disrupts the targeting and stabilization of the basket axon collaterals at the pinceau and does not affect Purkinje and basket neuron synapse formation. 


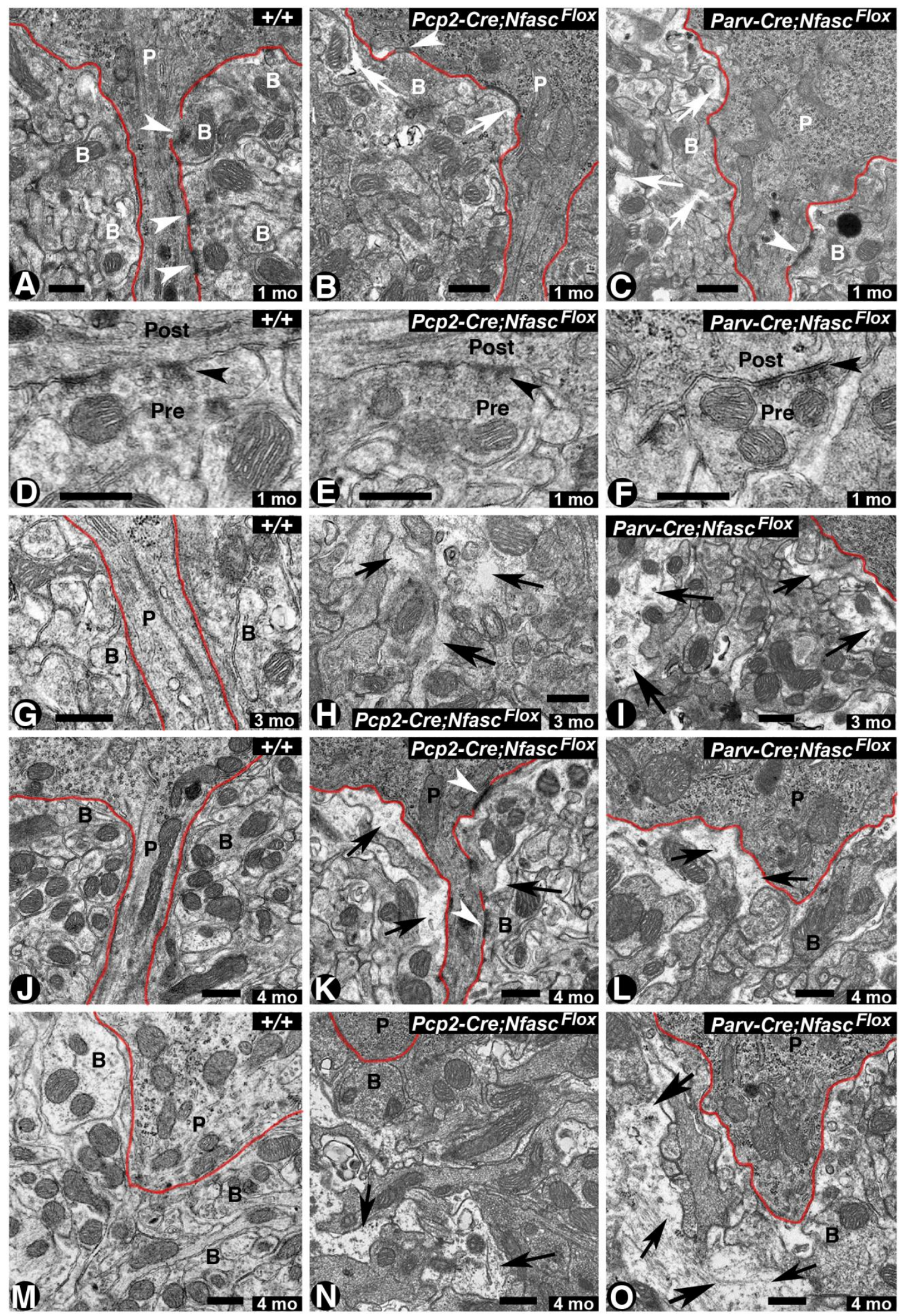


Figure 3.8 Cerebellar pinceau disorganization and infiltration of glial processes in Nfasc-deficient Purkinje and basket neuron cerebella.

(A-C) Electron micrographs of the pinceau region of cerebellar sections from one-month old wild type (+/+) pinceau show compact, electron-dense basket axon collaterals (B) surrounding the Purkinje soma/AIS highlighted by red line (A). Note the presence of synapses between Purkinje (P) AIS and basket collaterals (B) (A, arrowheads). In one month old Pcp2-Cre;Nfasc ${ }^{\text {Flox }}$ cerebella, the pinceau appears slightly disorganized, with spaces beginning to form between basket axon terminals (B) (B, arrows). Note the presence of a synapse between the basket terminal and the Purkinje soma (B, arrowhead). In one month old Parv-Cre; Nfasc ${ }^{\text {Flox }}$ cerebella the pinceau appears more disorganized with glial processes infiltrating spaces between basket axon terminals ( $\mathrm{C}$, arrows). (D-F) Electron micrographs at higher magnification showing synapses (black arrowheads) with presynaptic (basket terminal) and postsynaptic (Purkinje AIS) areas in the wild type (D), Pcp2-Cre;Nfasc ${ }^{\text {Flox }}$ (E) and Parv-Cre;Nfasc ${ }^{\text {Flox }}$ (F) cerebella. (G-I) Electron micrographs from cerebellar sections from 3-month old wild type cerebella show highly compact basket terminals surrounding the Purkinje AIS (G). Pcp2Cre; fasc $^{\text {Flox }}$ pinceau at 3 months shows increased infiltration of glial processes $(\mathrm{H}$, black arrows). A 3 month old Parv-Cre; fasc $^{\text {Flox }}$ pinceau shows increased disorganization of basket terminals surrounding the Purkinje soma/AIS (I, black arrows). (J-O) Electron micrographs of the pinceau from 4 month old wild type (J, M), Pcp2Cre; $\operatorname{Nfasc}^{\text {Flox }}(\mathrm{K}, \mathrm{N})$ and Parv-Cre;Nfasc ${ }^{\text {Flox }}(\mathrm{L}, \mathrm{O})$ cerebella. Wild type pinceau show compact basket axon terminals surrounding the Purkinje soma/AIS. The Pcp2Cre;Nfasc ${ }^{\text {Flox }}$ and Parv-Cre;Nfasc ${ }^{\text {Flox }}$ cerebella show severely disorganized basket terminals with increased infiltrating glial processes (black arrows). In some instances, in Parv-Cre; $\mathrm{Nfasc}^{\text {Flox }}$ cerebella the entire pinceau areas are completely disrupted (O, black arrows). Scale bars: $0.5 \mu \mathrm{m}$ 


\subsubsection{Nfasc-Deficient Purkinje Neurons Lack Spontaneous Activity and Receive Reduced Inhibitory Input from Basket Neurons}

Since loss of Nfasc resulted in a failure to assemble AIS molecular components, and also caused mistargeting of the basket axon collaterals at the Purkinje AIS, we wanted to address how loss of Nfasc affected the electrophysiological properties of the Purkinje neurons. In voltage-clamp mode, we tested if the spontaneous activity, defined as firing rate in the absence of any current injection, of Pcp2-Cre; $\mathrm{Ffasc}^{\text {Flox }}$ mutant Purkinje neurons was different from the control Purkinje neurons (Fig. 3.9A-B). In the control group, 13 of 19 wild type neurons $(68.4 \%)$ produced action potentials spontaneously, while only 6 of 18 Pcp2-Cre; fasc $^{\text {Flox }}$ neurons (33.3\%) fired spontaneously, the rest of them remaining silent (Fig. 3.9A). The mean firing rate across all wild type neurons was significantly different from the mean firing rate across all Pcp2-Cre;Nfasc ${ }^{\text {Flox }}$ mutant neurons $(24.6+/-5.6$ spikes/ sec, $\mathrm{n}=19$, and $7.7+/-3.3$ spikes/sec, $\mathrm{n}=18$, respectively, $\mathrm{p}=0.02$ ) (Fig. 3.9B). Comparing only the spontaneously

active neurons, wild type neurons fired at 36.1 +/- $5.9 \mathrm{~Hz}(\mathrm{n}=13)$ and Pcp2-Cre; Nfasc ${ }^{\text {Flox }}$ mutant cells fired at $23.2+/-6.1 \mathrm{~Hz}(\mathrm{n}=6, \mathrm{p}=0.17)$. No significant difference in the mean amplitude of action potentials was noticeable (control: $60.4+/-2.5 \mathrm{mV}, \mathrm{n}=13$, Pcp2Cre; fasc $^{\text {Flox }}$ mutant: $\left.61.0+/-2.9 \mathrm{mV}, \mathrm{n}=6\right)(\mathrm{p}=0.96)$. We hypothesized that the silent cells had a more hyperpolarized resting potential, preventing their depolarization to threshold. However, the resting potential of the 12 silent Pcp2-Cre;Nfasc ${ }^{\text {Flox }}$ mutant neurons $(-53.3+/-1.8 \mathrm{mV})$ was not significantly more hyperpolarized than the resting potential of the 13 spontaneously active control neurons $(-50.5+/-0.6 \mathrm{mV}, \mathrm{p}=0.14)$ (Fig. 3.9B). Thus, the trend to decreased spontaneous firing rate in the mutant neurons is not 
caused by a more hyperpolarized resting potential. Furthermore, no significant differences in neuronal input resistance, series resistance or capacitance was detected between the control and Pcp2-Cre; Nfasc ${ }^{\text {Flox }}$ genotypes (data not shown), indicating that the active membrane properties of mutant and wild type neurons were similar.

Next, to further test the firing properties of Pcp2-Cre;Nfasc ${ }^{\text {Flox }}$ mutant Purkinje neurons, we recorded action potentials triggered by depolarizing current steps in both control and Pcp2-Cre;Nfasc ${ }^{\text {Flox }}$ mutant Purkinje neurons (Fig. 3.9C-F). In current-clamp mode, cells were held at $-70 \mathrm{mV}$, and $500 \mathrm{msec}$ steps of 100 to $800 \mathrm{pA}$ were applied (by increments of $100 \mathrm{pA})$. Characteristic responses of wild type and Pcp2-Cre;Nfasc Flox mutant cells to 200, 400, 600 and 800 pA injections are illustrated in Fig. 3.9C. All cells responded to depolarizing injections with a train of action potentials, a single spike, or a burst. The mean threshold of current injection at which cells started to fire was not significantly higher in mutants than in control cells (control: $268.4+/-40.5 \mathrm{pA}, \mathrm{n}=19$, mutant: $427.8+/-75 \mathrm{pA}, \mathrm{n}=18, \mathrm{p}=0.23)$, indicating that Pcp2-Cre;Nfasc ${ }^{\text {Flox }}$ mutant cells are able to start to fire for similar intensity of depolarization. The number of spikes during each step was counted (Fig. 3.9D). The sustained firing rates were quantified as the number of spikes divided by the time between the first and the last spike; the firing rate for steps eliciting only a single spike was scored as 0 spikes/sec. Although the sustained firing rate was not different in control and mutant cells for every step (Fig. 3.9E), the number of spikes generated in mutant cells was significantly lower than in control cells for current injections above 300 pA. These data show that Pcp2Cre; fasc $^{\text {Flox }}$ mutant cells are able to fire repetitively like the control cells, but they are unable to fire more than about 10 spikes, resulting in a lower spike number (Fig. 3.9F). 
Because the loss of Nfasc expression results in altered targeting of the basket axon collaterals, we investigated the possibility that inhibitory inputs could be impaired in Pcp2-Cre; $N f a s C^{\text {Flox }}$ mutant Purkinje neurons. In the current-clamp mode, we recorded sIPSCs in Purkinje cells, in the presence of glutamatergic antagonists (NBQX and APV). In Pcp2-Cre; Nfasc ${ }^{\text {Flox }}$ mutants, the frequency of sIPSCs was significantly decreased, compared with control cells (WT: $1.5+/-0.2 \mathrm{~Hz}, \mathrm{n}=5$; Pcp2-Cre; ;fasc ${ }^{\text {Flox }}$ mutant: $0.2+/$ $0.04 \mathrm{~Hz}, \mathrm{n}=9, \mathrm{p}=0.001)$. The amplitude of sIPSCs was not significantly changed in $P c p 2-$ Cre; Nfasc ${ }^{\text {Flox }}$ mutant cells compared with control cells (WT: $32.1+/-3.4 \mathrm{pA}, \mathrm{n}=5 ;$ Pcp2Cre; Nfasc $^{\text {Flox }}$ mutant: $28.5+/-4.9 \mathrm{pA}, \mathrm{n}=9, \mathrm{p}=0.52$ ). The reduction in the frequency of inhibition observed in Pcp2-Cre; $N f a s C^{\text {Flox }}$ mutants in the sIPSCs recordings indicates that inhibitory inputs are impaired in mutant cells. To determine whether the decreased sIPSC reflected a decrease in spontaneous action potential firing in basket neurons or a decrease in the number or release probability of inhibitory inputs, we recorded mIPSCs in the presence of TTX $(1 \mu \mathrm{M})$ and glutamatergic antagonists (Fig. 3.9G-H). No significant difference was found in the mIPSC amplitude (WT: $27.4+/-4.4$ pA, n=11; Pcp2Cre; Nfasc $^{\text {Flox }}$ mutant: $35.5+/-6.4, \mathrm{n}=13, \mathrm{p}=0.57$; Fig. $\left.3.9 \mathrm{H}\right)$. However, the mIPSC frequency was decreased $[1.2+/-0.3 \mathrm{~Hz}$ in WT $(\mathrm{n}=11), 0.26+/-0.05 \mathrm{~Hz}(\mathrm{n}=13)$ in mutant cells ( $\mathrm{p}=0.026$ ] (Fig. 3.9H). These data indicate that inhibitory inputs onto Pcp2Cre;Nfasc ${ }^{\text {Flox }}$ mutant Purkinje neurons are decreased in number or have a decreased rate of presynaptic release. 


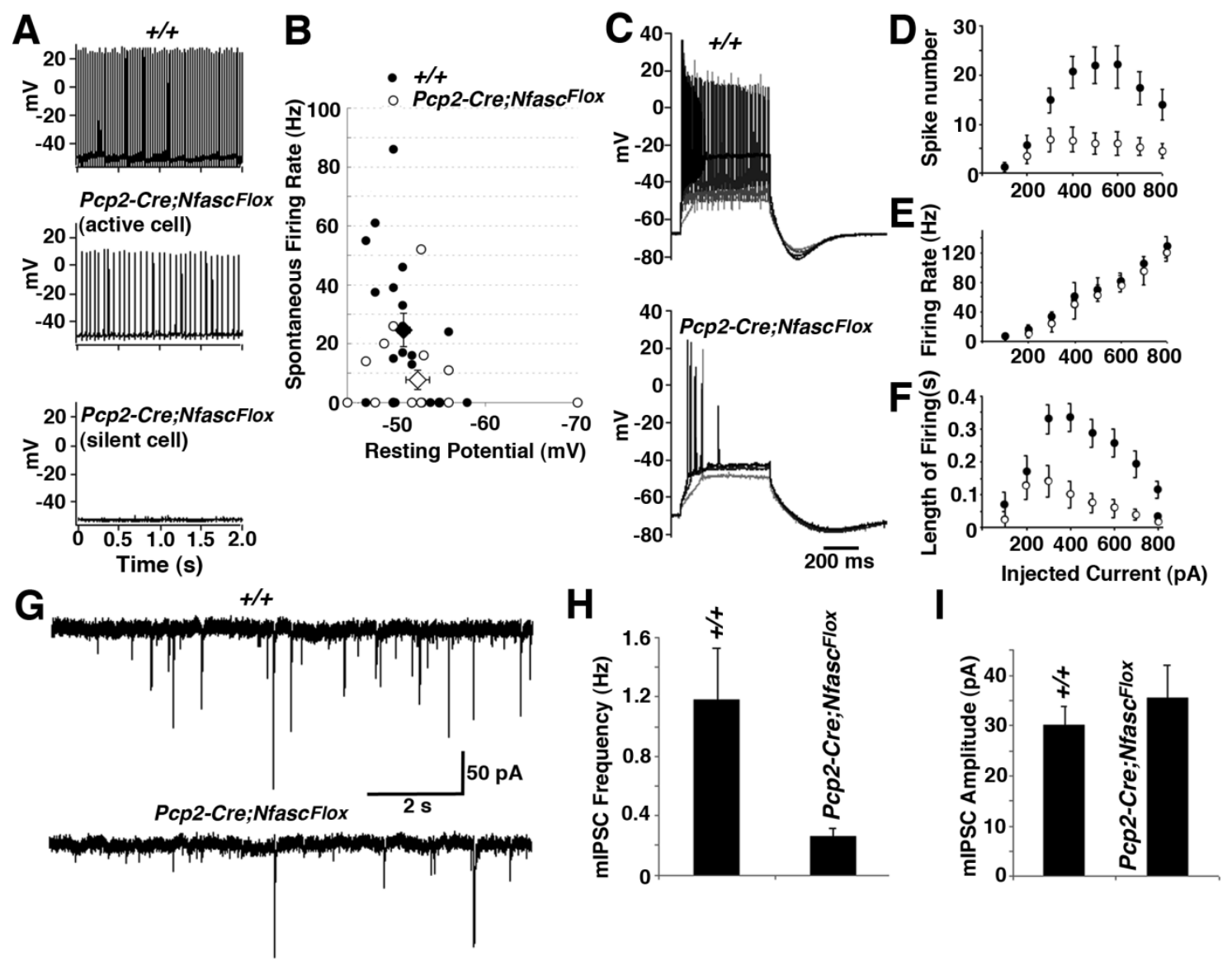

Figure 3.9 Purkinje neuron-specific ablation of Nfasc abolishes Purkinje neuron spontaneous activity and reduces inhibitory inputs to Purkinje neurons.

(A) Representative traces of spontaneous Purkinje neuron firing in wild type $(+/+)$ and Pcp2-Cre;Nfasc ${ }^{\text {Flox }}$ Purkinje neurons.

(B) Plot of the spontaneous firing rates of wild type (black circles) and Pcp2-

Cre; Nfasc ${ }^{\text {Flox }}$ mutant (white circles) Purkinje neurons. Larger diamonds show average spontaneous firing rates of Purkinje neurons in wild type (black diamond) and Pcp2Cre; Nfasc ${ }^{\text {Flox }}$ mutants (white diamond).

(C) Representative traces of the action potential train response of Purkinje neurons to injected current at increasing depolarizing steps.

(D-F) Output responses from wild type (black circles) and Pcp2-Cre;Nfasc ${ }^{\text {Flox }}$ (white circles) Purkinje neurons to increasing levels of injected current.

(G) Representative traces of mIPSC recordings from wild type and Pcp2-Cre;Nfasc Flox Purkinje neurons.

(H) Plot of mIPSC frequency reveals decreased frequency in Pcp2-Cre; fasc $^{\text {Flox }}$ mutant Purkinje neurons.

(I) Plot of mIPSC amplitude revealing no change between the wild type and Pcp2-

Cre; fasc $^{\text {Flox }}$ mutant Purkinje neurons. 
To test whether excitatory inputs were altered in response to the disrupted inhibitory input onto Purkinje neurons, we also tested the parallel fiber (PF) inputs onto Purkinje cells in Pcp2-Cre;Nfasc ${ }^{\text {Flox }}$ cerebella (Fig. 3.10). In voltage-clamp mode, PFEPSCs were recorded in Pcp2-Cre;Nfasc ${ }^{\text {Flox }}$ mutant cells and compared to wild type PFEPSCs. No significant difference between genotypes was noticed when the intensity of stimulation was increased, and the paired-pulse facilitation was similar in both groups for different tested delays, suggesting that PF connections are not affected pre- or postsynaptically by the abnormal inhibitory inputs and the disrupted AIS in mutant Purkinje neurons. Together, these results indicate that Purkinje neuron physiology is compromised with Nfasc ablation and inhibitory input onto Nfasc mutant Purkinje neurons is reduced. 

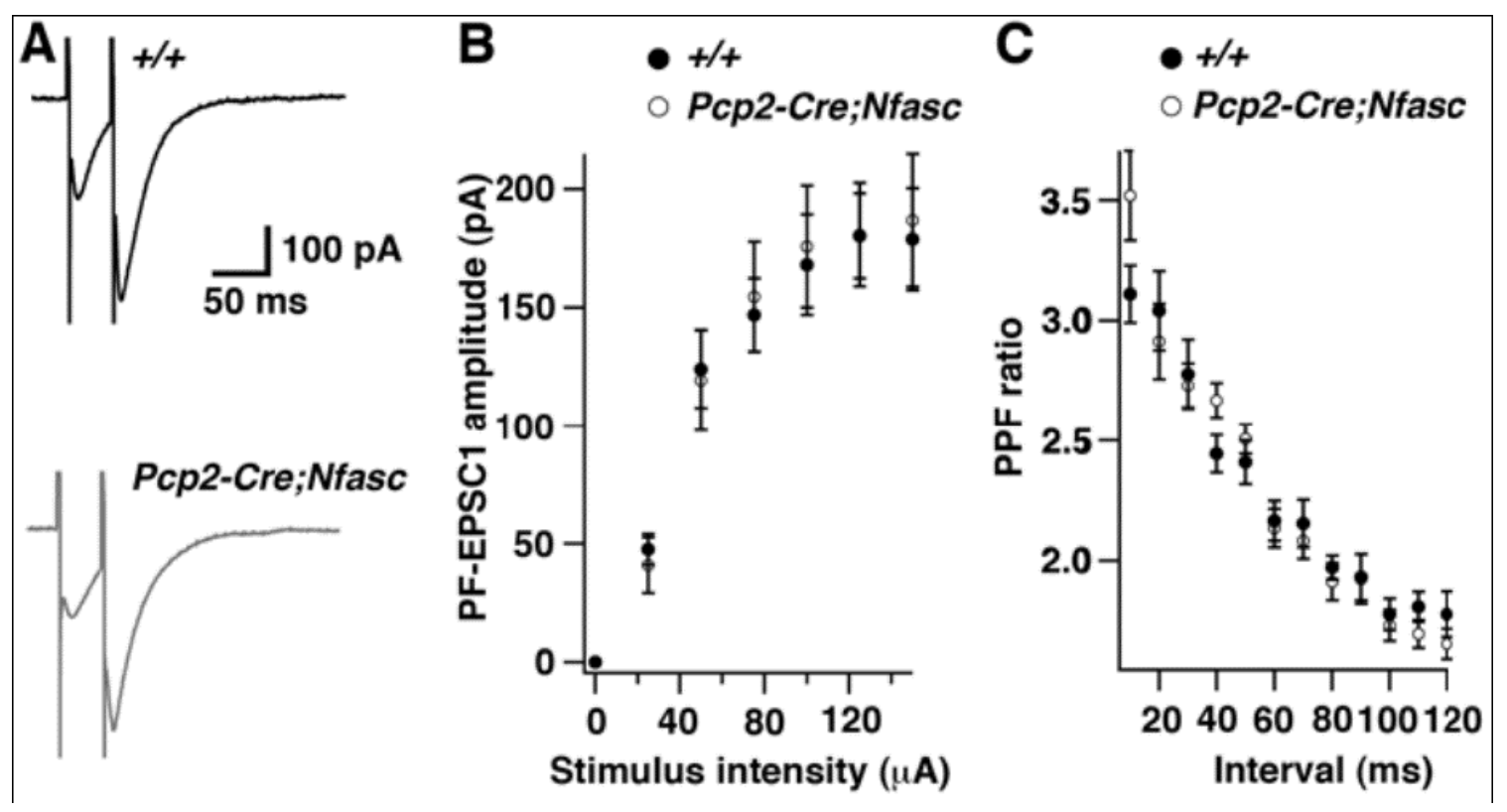

Figure 3.10 Parallel Fiber (PF) inputs are unchanged in Purkinje neuron-specific Nfasc mutants.

(A) Representative traces of PF-EPSCs in wild type (+/+) and Pcp2-Cre;Nfasc Flox Purkinje neurons.

(B) Plot of the PF-EPSC1 amplitude evoked in wild type (black circles, $\mathrm{n}=14$ ) and Pcp2Cre;Nfasc ${ }^{\text {Flox }}$ mutant (white circles, $\mathrm{n}=9$ ) Purkinje neurons with increasing intensity of stimulation.

(C) Paired pulse ratio of PF-EPSCs from wild type (black circles, $\mathrm{n}=16$ ) and Pcp2-

Cre;Nfasc ${ }^{\text {Flox }}$ (while circles, $\mathrm{n}=10$ ) Purkinje neurons to increasing stimulation intervals. 


\subsubsection{Loss of Nfasc in Purkinje and Basket Neurons Leads to Progressive Ataxia and Neurodegeneration}

The synaptic circuitry of the cerebellum relies on the Purkinje neurons as the sole output source. Thus, Purkinje neuron dysfunction is thought to be the major contributing factor in most ataxias and motor coordination deficits (Manto and Marmolino, 2009; Zoghbi, 2000). Since loss of Nfasc in Purkinje neurons leads to Purkinje AIS and pinceau disorganization, we wanted to determine whether Pcp2-Cre;Nfasc ${ }^{\text {Flox }}$ and ParvCre;Nfasc ${ }^{\text {Flox }}$ mice displayed any signs of motor incoordination and ataxia and Purkinje neuron degeneration. We first performed rotarod tests as a measure of motor coordination on Pcp2-Cre; $\mathrm{Nfasc}^{\text {Flox }}$ mice. These mice became progressively more ataxic and showed decreased latency to fall from the accelerating rotarod as they aged (Fig. 3.11B). We also utilized the Pcp2-Cre; $\mathrm{Nfasc}^{\mathrm{Flox}}$ mice in an open field test to examine their locomotor activity levels. We found that the Pcp2-Cre;Nfasc ${ }^{\text {Flox }}$ mice were always less active than the wild type mice and these differences became more pronounced with age, until the mutant mice barely moved at all during the 60-minute test by age 13weeks old (Fig. 3.11C). Interestingly, the ataxia is significant in these mutants weeks after the Purkinje AIS and pinceau are anatomically disrupted, based on our immunohistochemistry (refer Figs. 3.4 \& 3.5). The Parv-Cre; Nfasc ${ }^{\text {Flox }}$ mice were not utilized for rotarod or open field tests as they displayed extremely severe motor deficits, owing to loss of Nfasc in other areas of the nervous system where Parv-Cre is expressed. It is important to note that Pcp2-Cre could also be expressed in other areas of the nervous system and this may affect the behavior of the animal. Visually, at around P90, the Pcp2-Cre; Nfasc ${ }^{\text {Flox }}$ mice 
begin to display motor deficits, including a wide-based gait and stumbling in the cage. As these mice age, these phenotypes become more severe.

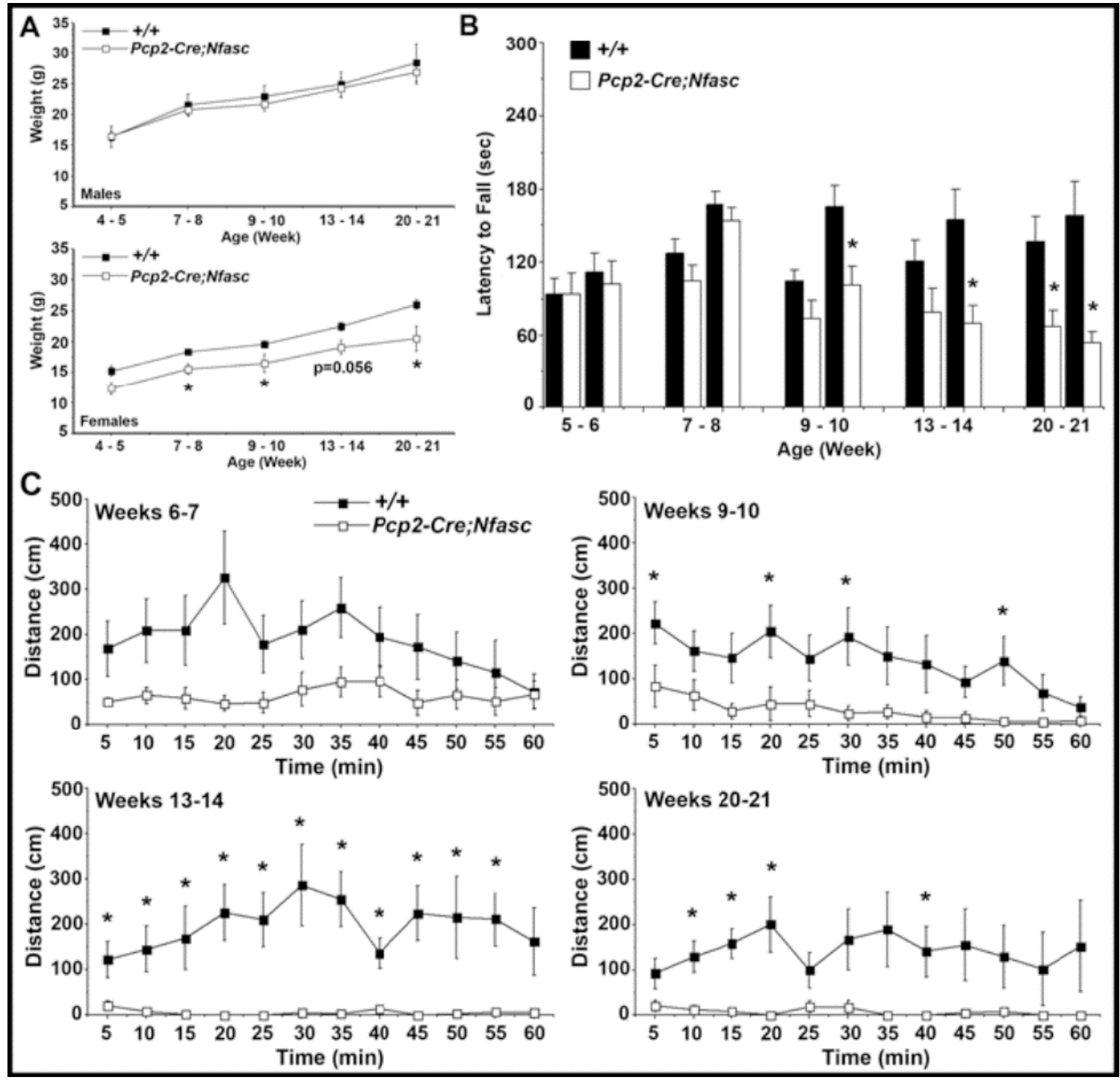

Figure 3.11 Purkinje neuron-specific loss of Nfasc results in ataxia.

(A) Weights of mice (in grams) during behavioral testing. Data shown are mean $( \pm$ SEM) for each group. $*<0.05$.

(B) Latency to fall from an accelerating rotarod. Pcp2-Cre; $f f a s c^{\text {Flox }}$ mice show decreased latency to fall as they age. Data shown are means $( \pm$ SEM) for each group. $*<0.05$.

(C) Total distance traveled in a novel environment. Pcp2-Cre;Nfasc ${ }^{\text {Flox }}$ mice are less active than wild type mice and their activity decreases with age. Data shown are means $( \pm$ SEM) for each group for a one-hour test session. ${ }^{*} \mathrm{p}<0.05$. 
To determine whether older Pcp2-Cre;Nfasc ${ }^{\text {Flox }}$ mice undergo neurodegeneration in the cerebellum, we carried out triple immunostaining of the cerebella from one year old (P365) wild type and Pcp2-Cre;Nfasc ${ }^{\text {Flox }}$ mutant littermates against Calb, Parv and $\mathrm{K}_{\mathrm{V}} 1.2$ to assess the loss of Purkinje and basket neurons, as well as the phenotypes at the pinceau in older mutants. As shown in Fig. 3.12A, B, the wild type cerebellum displays a well-ordered Purkinje neuron layer (Fig. 3.12Aa, d; Ba, d; white arrows), presence of basket interneurons (b) (Fig. 3.12Ab, d; Bb, d, red arrow) and normal shape pinceau (Fig. 3.12Ac, d; Bc, d; blue arrowhead). In contrast, the Pcp2-Cre;Nfasc ${ }^{\text {Flox }}$ mutant cerebellum has essentially lost all its Purkinje neurons (Fig. 3.12Ca, d; Da, d; white arrows) and the molecular layer has been reduced in size to almost one third of that of the wild type (compare Fig. 3.12Cd with 3.12Ad). The Calb immunoreactivity is essentially absent in Pcp2-Cre;Nfasc ${ }^{\text {Flox }}$ mutant cerebella, except in some background cell nuclei (Fig. 3.12Da, d; green arrows). Basket neurons (b) are present and their axon collaterals form bundles (Fig. 3.12Db; red arrowheads) with traces of $\mathrm{K}_{\mathrm{V}} 1.2$ immunoreactivity reflecting the disorganized pinceau (Fig. 3.12Dc; blue arrowheads). Similarly, we analyzed cerebella from 4 1/2 month old Parv-Cre;Nfasc ${ }^{\text {Flox }}$ mutant mice, which also revealed Purkinje neuron degeneration (Fig. 3.12Ga; Ha; white arrows). The basket neurons (b) are still present (Fig. 3.12Gb; Hb), but their axon collaterals are mistargeted and display severely disorganized pinceau structures (Fig. 3.12Gc; Hc; blue arrowheads). The ParvCre; Nfasc $^{\text {Flox }}$ mutant mice become extremely uncoordinated as they reach $\sim 6$ months of age and rarely survive past this age. At this point they also display more Purkinje neuron degeneration (data not shown). To quantify the levels of Purkinje neuron degeneration that occurs before the Parv-Cre;Nfasc ${ }^{\text {Flox }}$ mice die, we counted the number of Purkinje 
neurons in cerebellar loops I, II, III, IV, V, VIa, VIb, and VIII from several cerebellar sections from each of five separate mice for both wild type and Parv-Cre;Nfasc ${ }^{\text {Flox }}$ and averaged the total number of Purkinje cells per loop. We found that there were an average of 161.9 Purkinje cells per loop in wild type cerebella at 5 months $(n=43$ loops from 5 separate animals) and 97.3 Purkinje cells per loop in Parv-Cre; fasc $^{\text {Flox }}$ cerebella at 5 months ( $\mathrm{n}=79$ loops from 5 separate animals) $(\mathrm{p}=0.00000024)$. This is a $40 \%$ decrease in Purkinje neurons by the time the Parv-Cre;Nfasc ${ }^{\text {Flox }}$ reach 5 months of age. These data show that loss of Nfasc in Purkinje neurons leads to progressive ataxia and Purkinje neuron degeneration. Together, the data presented in this study reveal critical roles for Nfasc in both Purkinje and basket neurons for proper basket axon outgrowth, organization of the pinceau, maturation of the Purkinje AIS, and thus cerebellar function. 

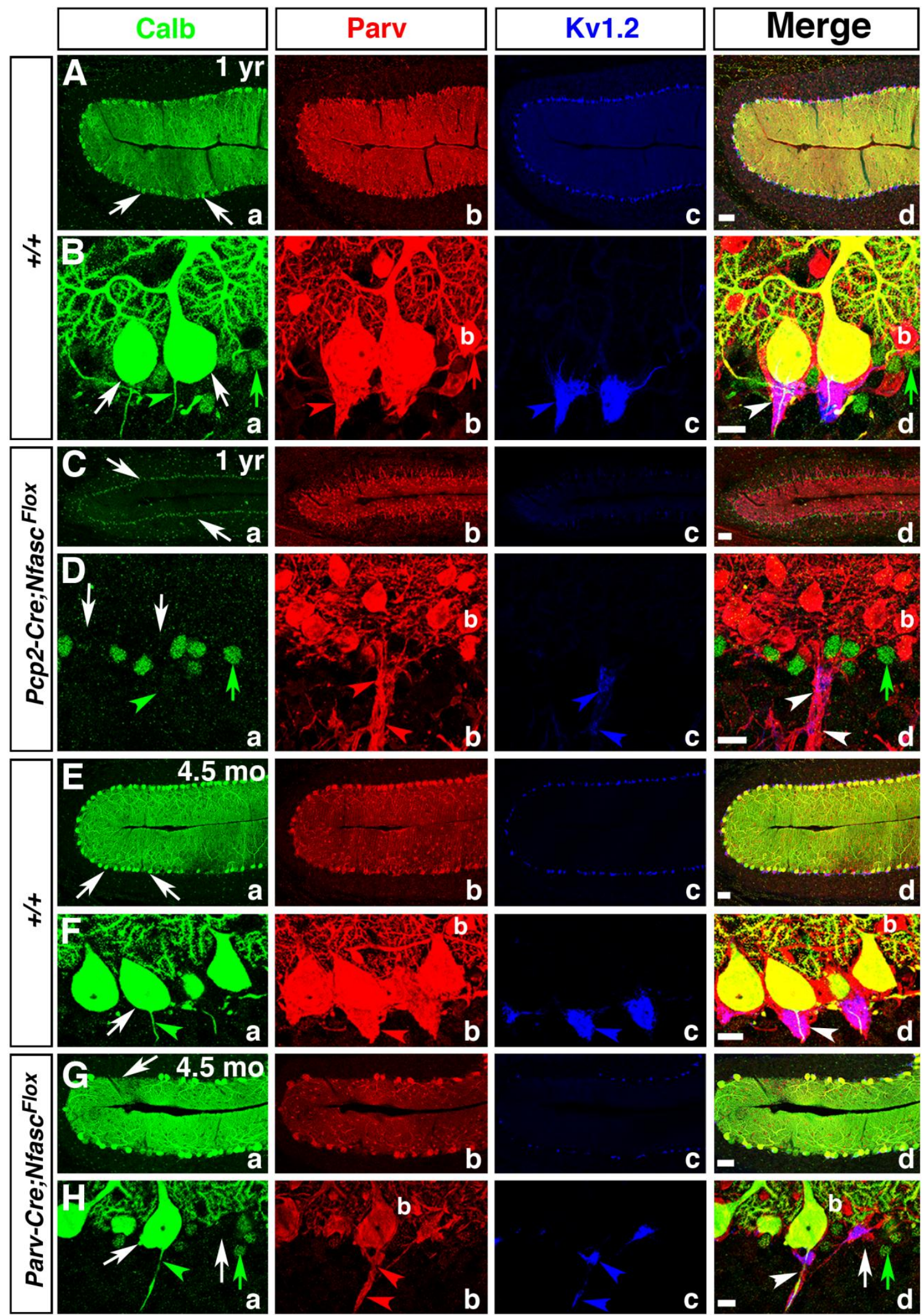

b
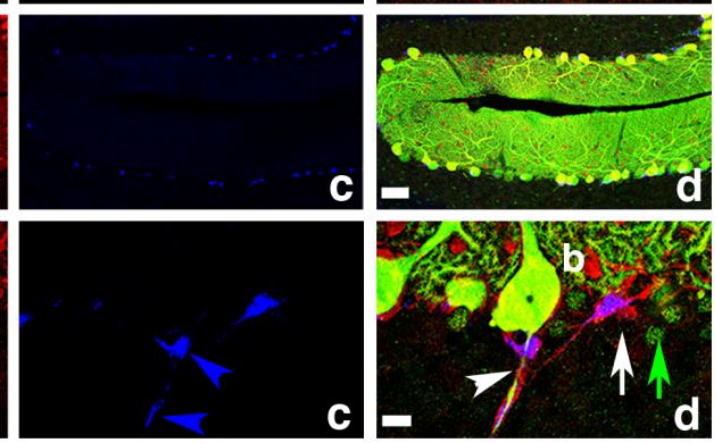
Figure 3.12 Ablation of Nfasc in Purkinje neurons leads to progressive Purkinje neuron degeneration.

One-year old wild type (A, B), one-year old Pcp2-Cre;Nfasc Flox (C, D), 4.5-month old wild type (E, F) and 4.5-month old Parv-Cre;Nfasc ${ }^{\text {Flox }}(\mathrm{G}, \mathrm{H})$ cerebellar sections immunostained against Calb (a, green), Parv (b, red), $\mathrm{K}_{\mathrm{V}} 1.2$ (c, blue) and merged (d). One-year and $4 \frac{1}{2}$ month wild type cerebella maintain a full complement of healthy Purkinje neuron layer (Aa, Ba, Ea, Fa, arrows), while one-year old Pcp2-Cre; Nfasc $^{\text {Flox }}$ cerebella show decreased molecular layer size with loss of Purkinje neurons (Ca, Da, arrows). The pinceau is stably maintained in one-year and $4 \frac{1}{2}$ month old wild type cerebella (Bb-d, Fb-d, arrows). In Pcp2-Cre; fasc $^{\text {Flox }}$ cerebella, the basket axons appear clumped together ( $\mathrm{Db}, \mathrm{d}$, arrowheads), but do not form a pinceau structure as all Purkinje neurons have died (Da, arrows). In 4 1⁄2 month Parv-Cre; $N$ fasc ${ }^{\text {Flox }}$ cerebella, some Purkinje cells have begun to degenerate ( $\mathrm{Ga}, \mathrm{d}$; Ha, d, arrows) and the pinceau structure remains disrupted with ectopic clusters of potassium channels (Hb-d, arrowheads). Scale bars: $50 \mu \mathrm{m}--\mathrm{A}, \mathrm{C}, \mathrm{E}, \mathrm{G} ; 10 \mu \mathrm{m}-\mathrm{B}, \mathrm{D}, \mathrm{F}, \mathrm{H}$. 


\subsection{Discussion}

The molecular organization of the cerebellar pinceau has intrigued neuroscientists since Cajal's drawings of the cerebellum first appeared (Ramon y Cajal, 1894). This intrigue has heightened with observations that normal pinceau organization is required for normal motor behaviors (Bobik et al., 2004; Xie et al., 2010). The physiological function of the pinceau in modulating Purkinje neuron output remains to be understood (Bobik et al., 2004; Huang et al., 2007). Here we used cell-specific Cre recombinase to ablate Nfasc, revealing that Nfasc is expressed in both Purkinje and basket neurons, and that Nfasc function is required in both neurons for pinceau organization. Importantly, we demonstrate that loss of Nfasc does not abolish pinceau synapse formation. Instead, basket axon collaterals are mistargeted leading to pinceau disorganization.

Electrophysiological analyses revealed a decreased probability of spontaneous firing and failure to maintain evoked action potential bursts in Nfasc mutant Purkinje neurons.

Together, our studies establish that Nfasc is required in both Purkinje and basket neurons for proper basket axon outgrowth and targeting for pinceau organization.

\subsubsection{Neurofascin in Adhesive Interactions Between Purkinje and Basket Neurons}

A key finding of this study is that Nfasc expression in both Purkinje and basket neurons may establish intercellular adhesion, either homophilically or heterophilically, to allow for stable interactions between the Purkinje soma/AIS and basket axon collaterals. While the role of Nfasc in AIS formation and stability is known (Hedstrom et al., 2008; Hedstrom et al., 2007; Zonta et al., 2011), Nfasc function in basket neurons is unknown. Our ultrastructural analysis showed that pinceau synapses formed in Parv-Cre;Nfasc ${ }^{\text {Flox }}$ 
mice, indicating that Nfasc is not critical for synapse formation (Fig. 3.8). CAMs similar to Nfasc have been shown to play a critical role in axon guidance (Shiga et al., 1993; Wiencken-Barger et al., 2004) and in establishing intercellular adhesion between extending axons and their target cells (Rader et al., 1993; Ushkaryov et al., 1992). Based on the abnormal branching of basket axon collaterals in Parv-Cre;Nfasc ${ }^{\text {Flox }}$ cerebella, Nfasc is needed for proper outgrowth and guidance of basket axons towards the Purkinje soma/AIS, and similarly, based on the extensive branching of the basket terminals around the Purkinje soma/AIS in Pcp2-Cre;Nfasc ${ }^{\text {Flox }}$ cerebella, Nfasc is involved in bidirectional, mostly heterophilic, interactions between the Purkinje soma/AIS and basket collaterals to stabilize the pinceau. Possible binding partners for Nfasc at the pinceau may include $\mathrm{NrCAM}$, which localizes to the Purkinje soma/AIS. However, NrCAM ablation does not significantly disrupt pinceau organization (Fig. 3.5), but coordinated loss of NrCAM and Nfasc may lead to pinceau disruption. Another potential binding partner of Nfasc at the AIS/pinceau is the $\beta$-subunit of $\mathrm{Na}_{\mathrm{V}}$ channels, which functions as a CAM, and has been shown to stimulate neurite outgrowth (Davis et al., 2004; Isom, 2002; Koticha et al., 2006; McEwen and Isom, 2004). Nfasc may also interact with ECM proteins to aid in pinceau organization. Elucidating the molecular nature of the Nfasc binding partners at the pinceau will allow for a better understanding of how Nfasc functions in basket and Purkinje neurons during cerebellar pinceau organization. 


\subsubsection{Loss of Nfasc in Basket and/or Purkinje Neurons and Consequences on Pinceau Organization}

Our comparative analyses revealed that pinceau organization was more severely affected in Parv-Cre;Nfasc ${ }^{\text {Flox }}$ than in Pcp2-Cre; ffasc $^{\text {Flox }}$ cerebella, leading to several possible interpretations. First, the increased loss of Nfasc could contribute to the more severe phenotype in Parv-Cre; Nfasc ${ }^{\text {Flox }}$ cerebella such that the compounded loss of Nfasc in basket and Purkinje neurons allows for mislocalization of other molecular components critical for pinceau organization. Thus, when Nfasc is lost in Parv-Cre;Nfasc ${ }^{\text {Flox }}$ cerebella, these components are more disrupted than in Pcp2-Cre;Nfasc ${ }^{\text {Flox }}$ cerebella and the pinceau is further disorganized. Alternatively, if Nfasc function was only required at the Purkinje soma/AIS, then loss of Nfasc in Purkinje and Purkinje/basket neurons should lead to an identical phenotype. However, in Pcp2-Cre; $f f a s c^{\text {Flox }}$ cerebella, a smaller pinceau, based on localization of $\mathrm{K}_{\mathrm{V}}$ channels and PSD95 can form. These molecules are more disrupted in Parv-Cre;Nfasc ${ }^{\text {Flox }}$ cerebella. This suggests Nfasc functions in a trans heterophilic manner during pinceau organization. Further, basket axon collaterals target the Purkinje AIS better in Pcp2-Cre;Nfasc ${ }^{\text {Flox }}$ than in Parv-Cre;Nfasc ${ }^{\text {Flox }}$ cerebella, suggesting that Nfasc in basket axons interacts with another molecule/s at the Purkinje soma/AIS. In Pcp2-Cre; $N f a s c^{\text {Flox }}$ cerebella, that molecule/s is lost from the AIS in the absence of Nfasc. Our immunohistochemical results show that $\mathrm{Na}_{\mathrm{V}} 1.6$ channel, the marker of the mature AIS (Van Wart and Matthews, 2006), fails to become enriched at the Purkinje AIS. Thus, the primary function of Nfasc in Purkinje neurons is to ensure maturation and stabilization of the AIS molecules, including molecules that serve as receptors or ligands for basket neuron-expressed Nfasc. When Nfasc is also absent in 
basket neurons, the axon collaterals fail to target properly and are unable to stably interact with the Purkinje AIS.

The signaling mechanisms triggered by Nfasc in basket neurons remain to be determined. Alternative splicing and post-translational modifications of CAMs affect their interactions with downstream signaling effectors. For example, spatiotemporal control of functionally distinct isoforms of NCAM and L1 regulate their interactions with FGF tyrosine kinase receptors to modulate axon growth and guidance (Walsh and Doherty, 1997). Further, several CAMs induce neurite outgrowth by interacting with FGF receptors in growth cones (Doherty et al., 2000; Hansen et al., 2008). Importantly, Nfasc interacts with FGF receptors for neurite outgrowth, such that the cytosolic domain of Nfasc is critical for FGF receptor activation, while the extracellular domain is critical for regulating FGF receptor signaling in vitro (Kirschbaum et al., 2009). Our studies provide in vivo evidence that Nfasc is required for basket axon collateral outgrowth and targeting, and this may require receptor-mediated signal transduction pathways.

\subsubsection{Basket Neuron Inhibitory Input to Purkinje Neurons and the Role of Pinceau}

Nfasc functions in the organization of inhibitory synapses by clustering gephyrin at the future AIS in the developing hippocampus and in adult dentate gyrus stabilizes GABAergic synaptic components (Kriebel et al., 2011). Interestingly, only a few basket axon collaterals synapse with the Purkinje soma/AIS at the pinceau, while the remaining collaterals just surround the AIS (Palay and Palay, 1974; Somogyi and Hamori, 1976; Sotelo, 2008). Our patch-clamp recordings revealed that Pcp2-Cre;Nfasc ${ }^{\text {Flox }}$ Purkinje neurons show reduced spontaneous firing rates and failure to maintain depolarization- 
evoked high-frequency spike firing. These observations suggest that an intact AIS is not required for action potential initiation, but is essential for maintaining firing in response to current injection and for spontaneous firing. Similar observations were made in mice that lack Nav1.6 channels (Khaliq et al., 2003). Thus, the abnormal spike firing properties in Pcp2-Cre; fasc $^{\text {Flox }}$ Purkinje neurons are likely related to the mislocalization of $\mathrm{Na}_{\mathrm{v}} 1.6$ channels (Fig. 3.4). Recent studies by Zonta et al. (2011) also showed that ablation of Nfasc in adult Purkinje neurons results in silent Purkinje neurons that fail to maintain spontaneous firing. Together, these data confirm that Purkinje AIS is critical for Purkinje neuron spontaneous activity and maintaining action potentials.

What role does the pinceau play in Purkinje neuron activity? Our studies uncovered a 5-6 fold reduction in mIPSC frequency, but not mIPSC amplitude, suggesting that the number of synaptic release sites, or their release probability, is decreased. This is consistent with our EM analysis, which revealed the presence of synapses between basket axons and Purkinje AIS, but fewer basket axon collaterals around the Purkinje AIS. Interestingly, mutations in Kcna2, which encodes basket axon collateral-specific $\mathrm{K}_{\mathrm{V}} 1.2$, also resulted in defective inhibitory input to Purkinje neurons (Xie et al., 2010). Together, these data suggest that pinceau plays an important role in modulating Purkinje neuron activity.

\subsubsection{Ataxia and Neurodegeneration in Nfasc Mutants}

Many genetic mutations are associated with Purkinje neuron degeneration and cerebellar dysfunction leading to ataxia (Perkins et al., 2010; Rinaldo and Hansel, 2010; Wang and Zoghbi, 2001). In this study, the Pcp2-Cre; ffasc $^{\text {Flox }}$ mice developed ataxia, 
consistent with the Purkinje neuron loss found in older Pcp2-Cre; fasc $^{\text {Flox }}$ cerebella, though possibly also due to ablation of $\mathrm{Nfasc}$ in other Pcp2-Cre-expressing neurons. Importantly, the ataxia does not develop in the Pcp2-Cre;Nfasc ${ }^{\text {Flox }}$ mice until weeks after the AIS and pinceau defects are found (refer Figs. 3.4, 3.5, \& 3.11). It is possible that the Purkinje neurons are able to compensate for disrupted function for some period of time and only once they begin to degenerate is their function truly compromised. In addition, the behavior of the animal is the result of the entire nervous system, and thus other parts of the motor circuitry may be able to compensate for cerebellar dysfunction for a short time. Thus, the precise mechanisms leading to ataxia in our mutant mice are not completely understood.

We are also left with the question of what might cause the Purkinje neuron death. Previous studies have shown that ion channels are essential for sustained high frequency firing by Purkinje neurons (Raman and Bean, 1997, 1999; Sacco et al., 2006; Zagha et al., 2008) and mutant forms of human channels are associated with cerebellar atrophy and spinocerebellar ataxias (Browne et al., 1994; Ophoff et al., 1996; Waters et al., 2006; Zhuchenko et al., 1997). A high density of ion channels at the Purkinje AIS is necessary for proper action potential propagation and as discussed above loss of Nfasc results in their mislocalization from the AIS, leading to Purkinje neuron dysfunction. Interestingly, mutations in Kcna2 also lead to ataxia suggesting that an intact Purkinje AIS is not sufficient to maintain normal Purkinje neuron firing, further highlighting an equally important role of the pinceau in ensuring proper Purkinje neuron output modulation (Xie et al., 2010). Additionally, Pcp2-Cre;Nfasc ${ }^{\text {Flox }}$ mice show progressive Purkinje neuron degeneration beginning around 7-8 months owing to secondary consequences. In several 
instances Purkinje neuron dysfunction has been attributed to glutamatergic excitotoxicity, so this could also contribute to Purkinje neuron loss (Perkins et al., 2010; Slemmer et al., 2005). Future studies in which Nfasc is only ablated in basket neurons may help differentiate Purkinje AIS versus basket Nfasc functions during pinceau organization and how inhibitory input to Purkinje neurons would be affected. In summary, we have provided in vivo evidence that cerebellar pinceau organization and function requires coordinated mechanisms involving distinct Nfasc functions in Purkinje and basket neurons. 


\section{Chapter 4}

\section{Discussion and Future Directions}

The work presented in this dissertation elucidates molecular components and mechanisms responsible for the organization and maintenance of axonal domains in myelinated axons, as well as the cerebellar pinceau. First, we showed that protein 4.1B is a critical component for paranode stabilization, helping to maintain the AGSJs. Second, we found that protein 4.1B is required for the organization of the JXP domain. Third, we revealed a role for Nfasc in the maturation of the AIS. Fourth, we confirmed that Nfasc localized to Purkinje neurons is critical for the stabilization of basket axons to form the cerebellar pinceau. Finally, we revealed a novel role for Nfasc in proper axon outgrowth during the formation of the pinceau. Together, these findings help define the mechanisms by which axonal domains in myelinated axons are formed and stabilized. However, many questions remain to be answered. Below is a discussion of the findings of this dissertation with a perspective of where it leaves the field, where we must go next, and how this may affect disease therapeutics. 


\subsection{Axo-glial septate junctions link myelinating glia to the axonal}

\section{cytoskeleton}

Over the past decade, many studies have contributed to our understanding of the mechanisms responsible for axonal domain organization in myelinated axons. These studies revealed that the strict localization of ion channels to unique axonal domains is required for action potential propagation. Importantly, these studies elucidated the fence function of the AGSJs (Bhat et al., 2001; Boyle et al., 2001; Pillai et al., 2009). Loss of the AGSJ fence also disrupts the organization of the axonal cytoskeleton (Garcia-Fresco et al., 2006). The work presented in this dissertation takes these studies a step further, showing that one of the links of the AGSJs to the underlying axonal cytoskeleton is protein 4.1B. When 4.1B is ablated, the paranodal AGSJs are able to form, but do not remain stabilized. At P30, the paranode is not compacted properly in the absence of 4.1B and eventually the AGSJs begin to fall apart. Thus, this work provides direct evidence for a molecular component involved in the regulation and stabilization of the paranodal AGSJs.

In addition, many studies have addressed the molecular organizers of the JXP domain. These studies found roles for Caspr2 and Tag1, but not PSD proteins, in the organization of the JXP (Horresh et al., 2008; Poliak et al., 2003; Rasband et al., 2002). Therefore, there was still a gap in the knowledge of the connection between the JXP membrane-bound proteins and the axonal cytoskeleton for stabilization of the JXP proteins. The work presented in this dissertation shows that protein $4.1 \mathrm{~B}$ is required for organization of the JXP domain. When 4.1B is ablated, the JXP fails to form, with no 
clustering of potassium channels, PSD95, or Caspr2. Thus, protein 4.1B acts as a molecular scaffold at both the paranode and JXP domains.

Within this dissertation we showed that paranode and JXP organization requires 4.1B up to four months by looking at the $\beta$-Act-Cre; $4.1 B^{\text {Flox }}$ mice. However, we still wanted to know how this disruption in domain organization affected the long-term health of the axon. Interestingly, our in-progress studies show that axons undergo axonal degeneration as they age in the absence of protein 4.1B. In these follow-up experiments, preliminary results show the presence of swellings in Purkinje axons of one year old $\beta$ Act-Cre $; 4.1 B^{\text {Flox }}$ cerebella (data not shown). This suggests that the axonal cytoskeleton is disrupted, resulting in axonal transport defects and accumulation of organelles in swellings along the axon, signs of axonal degeneration (Garcia-Fresco et al., 2006). Intriguingly, it appears that the CNS paranodes are not completely disrupted in the one year old $4.1 B$ mutants. This suggests that the AGSJs are able to undergo remodeling to re-cluster and form the paranode in the absence of 4.1B (Shepherd et al., 2012). However, the link from the AGSJs to the axonal cytoskeleton is still missing, resulting in disorganization of the axonal cytoskeleton and axonal swellings. The swellings reflect the role of the AGSJs in organizing the axonal cytoskeleton, which cannot maintain its proper structure without contacting the axonal membrane complexes.

In addition, the PNS paranodes appear to disorganize further as the $\beta$-ActCre $; 4.1 B^{\text {Flox }}$ mice age from four months to one year (data not shown). Overall, the paranodes appear more stretched out, with broken segments. These preliminary studies show that Caspr localization in 1-year $4.1 B$ mutant paranodes looks a lot like that of inducible ablation of fasc $^{N F 155}$ (Pillai et al., 2009). In this study, tamoxifen-induced 
ablation of $N f a s c^{N F 155}$ in the adult mouse leads to increasing loss of the paranode from the JXP side towards the nodal side until the paranode is completely disrupted. The phenotypes differ in that the JXP is completely disrupted in one year $\beta$-Act-Cre $; 4.1 B^{\text {Flox }}$ mice, whereas the JXP remains intact, albeit mislocalized towards the node, in the inducible ffasc $^{N F 155}$ knockouts (data not shown; Pillai et al., 2009). There are also places in the $\beta$-Act-Cre; $4.1 B^{\text {Flox }}$ axons where nodal AnkG protein appears to enter the broken gaps in paranodal Caspr localization (data not shown). Examination of 1-year $\beta$-ActCre $; 4.1 B^{\text {Flox }}$ spinal cord and sciatic nerves by EM will help us to definitively understand what is happening within the axon with long-term loss of protein 4.1B.

Together, the results presented in this dissertation regarding protein $4.1 \mathrm{~B}$ show that it functions much like the other 4.1 proteins to link the cellular membrane to the underlying cytoskeleton. Similar to the function of protein $4.1 \mathrm{R}$ in the maintenance of the shape of red blood cells (Shi et al., 1999), 4.1B is critical for maintaining the membrane organization of axonal domains in myelinated axons. Further, 4.1B function is conserved in 4.1 orthologs from vertebrates to invertebrates, where the protein Cora is critical for stabilizing the septate junction protein Nrx IV (Banerjee et al., 2006b; Baumgartner et al., 1996; Fehon et al., 1994; Lamb et al., 1998; Ward et al., 1998). Thus, protein 4.1B functions as a cytoskeletal adaptor to organize the JXP and to stabilize the paranodal AGSJs. 


\subsection{Future directions for elucidating new paranodal proteins important for AGSJ stabilization}

While we provide evidence for the role of protein 4.1B in the stabilization of AGSJs and organization of the JXP, the AGSJs are still able to form and do not immediately fall apart upon ablation of 4.1B. This suggests that other molecular players are involved in the stabilization of the AGSJs. We did not see a compensatory increase in protein 4.1R protein levels in $\beta$-Act-Cre $; 4.1 B^{\text {Flox }}$ sciatic nerves or spinal cords. However, this does not rule out the function of other 4.1 proteins in paranode stabilization. A recent paper showed that protein $4.1 \mathrm{G}$ plays an important role in the organization of the internode (Ivanovic et al., 2012). Protein 4.1G is localized to Schwann cells (Horresh et al., 2010), and this new study showed that $4.1 \mathrm{G}$ functions to maintain proper localization of ion channels at the JXP and inner mesaxon of the Schmidt-Lanterman incisures (Ivanovic et al., 2012). In addition to 4.1B, 4.1N and 4.1R have been shown to be expressed in PNS sensory neurons (Arroyo et al., 2004; Ogawa et al., 2006; Ohara et al., 2000; Poliak et al., 2001). Therefore, it is possible that even without a compensatory increase in the $4.1 \mathrm{~B}$ mutants, these other 4.1 proteins could play some role in axonal domain organization.

While 4.1 proteins are clearly important for axonal domain organization, there must be other components to the complex that links the AGSJs to the underlying axonal cytoskeleton. Because the c-terminus of Caspr is the only axonal intracellular component of the AGSJs, we assume unidentified proteins must interact with the Caspr c-terminus. Previous studies in the lab utilized a yeast-2-hybrid screen with the Caspr c-terminus to identify binding partners, and identified the protein Whirlin (Green and Bhat, 
unpublished studies). Whirlin contains three putative PDZ-binding motifs, suggesting it may be able to play a role in stabilization of membrane-bound complexes. In addition, whirlin has known roles for the coordination of defined cellular domains. For example, in the hair cells of the inner ear, whirlin interacts in complexes with the membraneassociated guanylate kinase (MAGUK) protein, p55, the MAGUK CASK, and 4.1 proteins, including $4.1 \mathrm{R}, 4.1 \mathrm{~N}$ and $4.1 \mathrm{~B}$, to regulate hair cell development and maintenance (Mburu et al., 2006; Okumura et al., 2010). Therefore, it is possible that whirlin, along with protein 4.1B, is needed for stabilization of the paranodal AGSJs. However, there still may be other proteins involved in this paranodal complex as well. AnkB and $\alpha \mathrm{II} / \beta \mathrm{II}$ spectrin are enriched at the paranode (Garcia-Fresco et al., 2006; Ogawa et al., 2006). Thus, it is possible these proteins form a complex with Caspr at the paranode to stabilize the AGSJs. Further evidence for this possibility comes from the ability of Caspr to co-immunoprecipitate with 4.1B, spectrin and actin (Garcia-Fresco et al., 2006).

In order to elucidate further novel binding partners for Caspr at the paranode, one could immunoprecipitate Caspr from spinal cord and sciatic nerve lysates and use mass spectrometry to identify binding partners and complex components. It will be important to use spinal cord and sciatic nerve tissue separately because the AGSJs may be stabilized through different mechanisms in the CNS versus PNS. In this dissertation we showed, for the first time, that different isoforms of protein 4.1B are expressed in the PNS, compared to the known isoforms in the CNS. These different 4.1B isoforms likely play different roles in the PNS versus CNS and suggest that the axonal domains are organized and stabilized through different mechanisms in the PNS and CNS. 
Finally, examination of $\beta$-Act-Cre; $4.1 B^{\text {Flox }}$ sciatic nerve EM images revealed a possible defect in the organization of the Schwann cell (data not shown). To investigate the possibility of a role for 4.1B in Schwann cell development, like that of $4.1 \mathrm{G}$, we have crossed the $4.1 B^{\text {Flox }}$ mice to Cnp-Cre, which is expressed in glial cells (Lappe-Siefke et al., 2003; Pillai et al., 2009). Initial examination of the sciatic nerves from the CnpCre $; 4.1 B^{\text {Flox }}$ mice at P30 reveal that Cnp-Cre expression is leaky in the axons and $4.1 \mathrm{~B}$ is missing in some axons and is present in others. Our immunohistochemical analyses shows that axons with 4.1B remaining appear normal, while axons missing 4.1B phenocopy the $\beta$-Act-Cre; $4.1 B^{F l o x}$ axons at this time point. However, we do not know if these axons are missing 4.1B in their myelinating glia. Because 4.1B expression is so abundant in the axon, it is impossible to differentiate glial versus axonal expression with immunohistochemistry alone. To parse this out, we could obtain Schwann cell cultures and run a western blot with the lysate from such a culture to see if $4.1 \mathrm{~B}$ is specifically expressed in Schwann cells. Another option is to use immuno-EM to look for the localization of immuno-labeled gold particles in the myelin sheath versus the axon. These studies will help elucidate how 4.1B is able to organize and stabilize the paranode and JXP domains.

Further exploration of the molecular components of the paranode will help us better understand how these structures are affected in disease states. For example, in MS we know that the nodal and paranodal domains are targeted by auto-antibodies, leading to disrupted node, paranode, and JXP domain organization (Coman et al., 2006; Howell et al., 2006; Mathey et al., 2007; Wolswijk and Balesar, 2003). However, key questions remain to be addressed that relate to repair and stabilization of these domains. The more 
we learn about how the paranode and other axonal domains are organized and stabilized, the more directed therapeutics can be developed in the future.

\subsection{Different mechanisms are responsible for AIS maturation versus initial formation}

The data presented in this dissertation provides evidence for distinct phases of AIS organization. First, AIS CAMs and ion channels become enriched at the AIS. This initial formation is followed by the maturation of the AIS, which includes the enrichment of the mature sodium channel isoform $\mathrm{Na}_{\mathrm{v}} 1.6$. Finally, the AIS is stabilized for long-term activity. The first step, AIS formation, is regulated by AnkG. Without AnkG, the rest of the CAMs and ion channels fail to become enriched at the AIS (Hedstrom et al., 2007; Zhou et al., 1998). The data presented in this dissertation reveal that the second step, AIS maturation, requires the function of Nfasc, such that loss of Nfasc results in failure of Nav1.6 to cluster at the AIS. We also confirmed that the stabilization of the AIS requires Nfasc (Zonta et al., 2011).

From these results, one can hypothesize that AIS organization occurs through specific and separate mechanisms that are required for distinct functions of the AIS. First, during AIS formation, a sieve forms that functions to regulate the proteins that are able to be transported into the axon. Next, during AIS maturation, the mature sodium channel isoform becomes clustered to the AIS for action potential initiation. One can speculate that AIS maturation is delayed so that the sieve at the AIS can be properly established first. Finally, the AIS is stabilized so that the sieve structure is maintained and proteins do not need to be constantly transported out of the axon. Thus, each step of AIS organization 
is regulated by a different mechanism and understanding these mechanisms may allow us to have a better understanding of how we might treat an injured AIS. In ischemic injury models, the AIS is specifically degraded when the spectrin and Ank proteins responsible for AIS organization and stabilization are proteolyzed by calpain (Schafer et al., 2009). This degradation could be prevented with the use of protease inhibitors. Because we know that maintaining the integrity of Ank and spectrins is important for maintaining AIS structure, a targeted approach for prevention could be elucidated. If we begin to understand the mechanisms responsible for AIS maturation and stabilization, we may be able to treat developmental- or disease-related AIS disorders.

\subsection{Future directions for elucidating the primary organizer of the AIS}

While AnkG is clearly required for initial clustering of the CAMs and ion channels that localize to the AIS, the molecular mechanisms responsible for AnkG enrichment at the AIS are unknown. As previously stated, it is thought that phosphorylated inhibitor of $\kappa \mathrm{B} \alpha(\mathrm{pI} \kappa \mathrm{B} \alpha)$ may function as a cofactor in AnkG trafficking to the AIS (Sanchez-Ponce et al., 2008; Schultz et al., 2006). One study showed that pI $\kappa \mathrm{B} \alpha$, activated inhibitory $\kappa \mathrm{B}$ kinase (IKK), and phosphorylated p65 are enriched at the AIS (Schultz et al., 2006). Phosphorylation of I $\mathrm{KB} \alpha$ results in activation of nuclear factor kappa-light-chain-enhancer of activated B cells (NF-kB), a transcription factor that helps neurons to avoid apoptosis (Barger et al., 1995; Bhakar et al., 2002; Kaltschmidt et al., 1999a; Kaltschmidt et al., 1999b). This phosphorylation depends on the activation of IKK (Mercurio et al., 1997). Thus, restriction of these activated proteins to the AIS suggests that the AIS plays a role in NF- $\kappa B$ signaling (Schultz et al., 2006). Interestingly, $\mathrm{pI \kappa B} \alpha$ 
associates with microtubules at the AIS and activated IKK associates with the membrane cytoskeleton at the AIS (Schultz et al., 2006). Another study showed that IKK activity and resulting phosphorylation of IкB $\alpha$ are necessary for AnkG localization to the AIS (Sanchez-Ponce et al., 2008). In addition, IKK activity is also required for axon formation (Sanchez-Ponce et al., 2008). Thus, it is possible that the initial mechanisms that specify the axon are involved in the clustering of AnkG at the AIS. The downstream targets of activated phosphatidylinositol 3-kinase (PI3-kinase), including Akt, glycogen

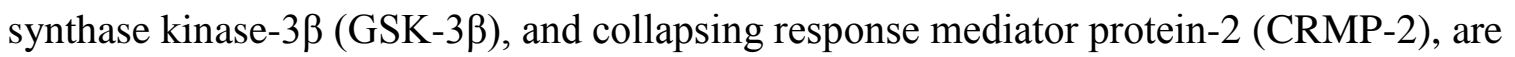
known to be involved in axon specification (Shi et al., 2003; Yoshimura et al., 2006a; Yoshimura et al., 2006b). There are several other pathways downstream of PI3-kinase that have been implicated in neuronal polarization. Interestingly, before axon specification, the actin cytoskeleton is unstable in the neurite that eventually becomes the axon (Bradke and Dotti, 1999). Further, application of actin destabilizing agents causes the formation of multiple axons (Bradke and Dotti, 1999). In addition, several studies have shown that the IKK and I $\mathrm{KB} \alpha$ pathways interact with other axon outgrowth pathways, including the PI3-kinase pathway (Alzuherri and Chang, 2003; Chen et al., 2001; Deng et al., 2004; Hacker and Karin, 2006; Perkins, 2007; Sanchez-Ponce et al., 2008; Wooten et al., 2000). The molecular players described here that are involved in axon specification function by organizing the axonal microtubule network. Therefore, it is possible these initial signals involved in microtubule organization also regulate the organization of $\mathrm{pI \kappa B} \alpha$ and IKK at the AIS and thus the activity of NF- $\mathrm{kB}$, which has been shown to be important for AnkG localization to the AIS. If similar mechanisms are responsible for axon specification and AIS initial organization, it may be difficult to 
differentiate the two. Future directions should be focused on downstream effects of NF$\kappa \mathrm{B}$ regulation that could be important in AIS organization. One way to do this would be to use RNASeq to look at how the transcriptome changes in neurons with disrupted IKK signaling. This may elucidate novel genes that are regulated by NF- $\kappa \mathrm{B}$ at the AIS.

It is likely that the organization of the axonal cytoskeleton at the AIS plays an important role in the initial assembly of the AIS. As previously mentioned, proteolysis of the spectrin/Ank cytoskeleton after ischemic injury results in disorganization of the AIS (Schafer et al., 2009). However, in vitro ablation of $\beta I V$-spectrin alone does not disrupt AIS organization (Hedstrom et al., 2007). Therefore, spectrin must play some other role at the AIS. It is possible that spectrin functions with actin to form and stabilize the sieve at the AIS. In addition, we wonder whether ablation of Nfasc disrupts the underlying axonal cytoskeleton when sodium channels and AnkG no longer localize to the AIS. To answer this question, future studies will examine the localization of $\beta I V$-spectrin at the Purkinje AIS of Pcp2-Cre;Nfasc ${ }^{\text {Flox }}$ and Parv-Cre;Nfasc ${ }^{\text {Flox }}$ cerebella. If $\beta \mathrm{IV}$-spectrin remains at the Purkinje AIS, while all other AIS components fail to remain enriched, then this will suggest that the axonal cytoskeleton at the AIS is organized by a different mechanism than the plasma membrane at the AIS. These results will answer the question of whether initial AIS organization is independent of the axonal cytoskeleton.

\subsection{Nfasc is required in both Purkinje and basket neurons for pinceau}

\section{organization}

The balance of excitation and inhibition in the brain is critical for maintaining proper neuronal function. The complex circuitry of the cerebellum requires a balance of 
communication between the cerebellar inputs (via climbing and mossy fibers), the cerebellar interneurons (granule, stellate, lugaro, and basket neurons), and the Purkinje neuron recurrent collaterals (Palay and Palay, 1974). Together, these inputs result in the Purkinje neuron receiving many inputs from both excitatory fibers and inhibitory interneurons (Palay and Palay, 1974). In addition, the Purkinje neuron regulates its own behavior by sending recurrent axon collaterals back to its soma and dendrites to function in feedback inhibition (Palay and Palay, 1974). The studies presented in this dissertation reveal that ablation of $N$ fasc results in disruption of these intricate circuits.

When Nfasc is ablated only in Purkinje neurons, the Purkinje AIS is not properly stabilized. As a result, the molecular cues responsible for binding with basket axon terminals are not properly stabilized at the Purkinje AIS/pinceau. Alternatively, Nfasc itself could be the cue required by basket axons to find the Purkinje soma/AIS and form the pinceau. We hypothesize that other molecular cues are also involved because some basket axons still reach the Purkinje soma/AIS area in both Nfasc mutants.

Importantly, when we ablated $N$ fasc from both Purkinje and basket neurons we found that the basket axon branches excessively and appears lost in its targeting (Fig 3.7). This suggests that Nfasc plays a role in regulating the outgrowth of the basket axon. It is possible that Nfasc is important for initiating signaling to the basket soma to communicate with the axon to stop branching. It is also possible that Nfasc interacts with the ECM or Purkinje soma to direct basket axon branching in the direction of the Purkinje AIS instead of into the molecular layer. As previously mentioned, several CAMs have been shown to function in axon outgrowth through the FGF receptor signaling pathway (Doherty et al., 2000; Hansen et al., 2008). This is one possible mechanism 
through which Nfasc may function at the pinceau. It is also possible that Nfasc functions through a novel pathway that other CAMs do not utilize. Studies have shown that members within the same receptor families do not always function the same way during development of the nervous system. For example, semaphorins are traditionally known to function as repulsive guidance cues during neuronal development (Mark et al., 1997). However, a more recent study found that semaphorin 7A functions to enhance axonal outgrowth through integrin receptors and the mitogen activated protein kinase (MAPK) pathway (Pasterkamp et al., 2003). Thus, it is possible that Nfasc functions through integrin receptors, or receptor tyrosine kinases such as the FGF receptor, to different intracellular signaling cascades, which may or may not include the MAPK pathway. Future studies, described below, are aimed to determine the precise mechanism of function Nfasc uses to regulate basket axon outgrowth.

\subsection{The pinceau modulates Purkinje neuron function}

Electrophysiological studies presented in this dissertation support the idea that the pinceau functions to inhibit Purkinje neuron activity. We found a decrease in mIPSC frequency, but not mIPSC amplitude, in Pcp2-Cre;Nfasc ${ }^{\text {Flox }}$ Purkinje neurons. This suggests that the number of basket synapses is decreased, but the quantal content is not affected. Because mIPSCs are measured in the presence of TTX to block action potentials, the decrease in mIPSC frequency could also be caused by a decrease in the frequency of spontaneous events in each basket terminal. One possible way to discriminate between these possibilities would be to do paired recordings in basket and Purkinje cells, which is a technically difficult experiment that relies on the chances of 
patching on a pair of cells that are in direct synaptic contact. By combining our electrophysiology data with the analysis of our immunohistochemistry, which shows decreased numbers of basket axon collaterals surrounding the Purkinje AIS in the Nfasc mutants, we hypothesize that a decrease in basket synapses likely results in the decreased mIPSC frequency phenotype. The function of the Purkinje neuron was also severely disrupted in Pcp2-Cre;Nfasc ${ }^{\text {Flox }}$ cerebella. However, the decrease in Purkinje neuron spontaneous and evoked firing is likely due to disruption in the Purkinje AIS, not disrupted inhibitory input onto the Purkinje neuron. The only way to differentiate these phenotypes would be to ablate $N f a s c$ in basket neurons alone and see how this affects Purkinje neuron activity.

Further support for the inhibitory role of the pinceau comes from histological studies that examined the localization of various ion channels at the pinceau. Interestingly, several studies found that there is a high density of several different voltage-gated potassium channel isoforms at the pinceau (Bobik et al., 2004; Laube et al., 1996). In addition, aquaporins, which localize to astrocytes, have been shown to be enriched at the pinceau (Bobik et al., 2004). Aquaporin channels are involved in potassium buffering, suggesting the aquaporins function with the high density of potassium channels at the pinceau (Gardner-Medwin, 1983, 1986). Together, these data suggest that the pinceau forms an electrical field potential that inhibits the Purkinje AIS (Bobik et al., 2004; Korn and Axelrad, 1980). In addition, the basket axon collaterals form septate-like junctions between themselves (Gobel, 1971). These so-called axoaxonic junctions can be seen in the EM data presented in this dissertation (refer Fig 3.8). Septate-like junctions function to separate and insulate domains between cells (Banerjee 
et al., 2006b). For example, the paranodal AGSJs function to separate the potassium channels in the JXP from the node and paranode (Bhat et al., 2001). Thus, the localization of potassium channels, aquaporin channels and axo-axonic junctions at the pinceau point to a mechanism in which an inhibitory electric field is produced that surrounds the Purkinje AIS and dampens Purkinje neuron function (Bobik et al., 2004). Importantly, the mechanism by which this electric field functions is not understood. It is possible that the high concentration of potassium ions at the pinceau help prevent depolarization of the AIS, thereby regulating action potential initiation. However, the precise function of potassium channels at the pinceau is unknown. Support for the role of potassium channels in pinceau function comes from a recent study in which a mutation in the potassium channel $\mathrm{K}_{\mathrm{V}} 1.2$ isoform was identified in a forward genetic screen (Xie et al., 2010). Interestingly, in these $\mathrm{K}_{\mathrm{V}} 1.2$ mutant mice, Purkinje neuron sIPSCs were increased resulting in decreased Purkinje neuron firing and ataxia. The authors suggest that the pinceau potassium channels are important for modulating basket axon firing and disruption in potassium channel function results in overactive basket neurons that overly inhibit Purkinje neuron function (Xie et al., 2010). The results from this study reiterate the importance of a precise balance of excitation and inhibition in cerebellar circuitry for regulating motor coordination.

\subsection{Purkinje neuron degeneration, ataxia, and cerebellar disease}

The cerebellum regulates motor coordination, so it is important to understand how it is functionally organized and regulated to better understand cerebellar diseases. Many disorders affect the function of the cerebellum. These include spinocerebellar ataxias and 
other movement disorders such as essential tremor. The spinocerebellar ataxias are caused by mutations in any one of 29-discovered genes, including the Ataxin gene (Brusco et al., 2004; Harding, 1993). Though these ataxias are all grouped under one name, they present with different phenotypes, which may include incoordination during fine motor tasks such as writing, buttoning a shirt, or eating, involuntary eye movements, clumsiness, poor speech, and tremors (Banfi and Zoghbi, 1994; Harding, 1993; Zoghbi, 1995, 2000). Importantly, pathology from these ataxias results from neurodegeneration of cerebellar Purkinje neurons (Manto and Marmolino, 2009; Zoghbi, 2000; Zoghbi and Orr, 2009). Genetically, spinocerebellar ataxias that affect the Ataxin gene are known as polyglutamine diseases, in that there is an increase of glutamines in the mutated genes (Zoghbi and Orr, 2009). In some cases of spinocerebellar ataxia, including spinocerebellar ataxia type 1 (SCA1), gain-of-function of Ataxin1 may be toxic to the Purkinje neuron leading to the pathogenesis (Zoghbi and Orr, 2009). Because there is still so much unknown about these disorders, it is critical to understand different mechanisms that lead to Purkinje neuron degeneration. Further, we need to understand which genes and proteins put the Purkinje neuron at risk for degeneration. The studies presented in this dissertation elucidate a route to Purkinje neuron degeneration whereby disorganization of axonal domains and altered function of inhibitory neurotransmission from the pinceau results in disrupted Purkinje neuron function. We hypothesize that the Purkinje neurons may be dying by way of glutamatergic excitotoxicity, due to the loss of basket neuron inhibitory input modulation and the decreased Purkinje neuron firing. Our preliminary follow-up studies suggest that vesicular glutamate transporter (vGlut), a marker of excitatory synapses, is increased in the cerebellar molecular layer of five 
month old Parv-Cre;Nfasc ${ }^{\text {Flox }}$ mice (data not shown). The presence of swellings along the Purkinje axon during earlier stages of Purkinje neuron dysfunction also point to the disruption of the axonal cytoskeleton (Garcia-Fresco et al., 2006). This is likely preventing normal axonal transport of materials required by the distal Purkinje axon to survive. Evidence for this was found in EM images that revealed the accumulation of organelles along the axon of three-month Parv-Cre;Nfasc ${ }^{\text {Flox }}$ Purkinje neurons (data not shown). Further, disruption of Purkinje neuron function in the $N$ fasc mutants may disrupt postsynaptic trophic support that the Purkinje neuron requires for survival. Together, these data highlight the importance of maintaining normal Purkinje neuron neurotransmission and regulated input onto the Purkinje neuron for Purkinje neuron survival and proper cerebellar function.

Another disorder that affects the ability of a patient to regulate motor coordination is essential tremor. The patient usually exhibits signs of tremors during movement, mostly in the arms and legs at first (Benito-Leon and Louis, 2007). Essential tremor can also affect the ability to walk and eventually may lead to dementia (Singer et al., 1994; Stolze et al., 2001). While some essential tremor cases are linked to genetic mutations, these represent only about half of the cases (Deng et al., 2007; Louis, 2001). Disease progression of essential tremor patients is thought to occur from an imbalance in the cerebellar network (Koster et al., 2002; Louis et al., 2007; Louis et al., 2009; Parisi et al., 2006; Singer et al., 1994). Autopsy of essential tremor patient cerebella revealed the presence of Purkinje axonal swellings and Purkinje cell death (Louis et al., 2007; Shill et al., 2008), much like what we see in our Nfasc mutants. Interestingly, recent findings have shown that abnormal basket axon branching appears to be a common phenotype of 
patients with essential tremor (Erickson-Davis et al., 2010). Further, there was an association in essential tremor patients with basket cell axons that were abnormally dense and tangled and Purkinje neuron degeneration (Erickson-Davis et al., 2010). This suggests that the proper branching of the cerebellar basket axon is critical for maintaining proper Purkinje neuron physiology and thus cerebellar function. Importantly, the authors point out that no transgenic mice exist to study essential tremor in vivo. Due to the abnormal basket axon branching found in the Parv-Cre; fasc $^{\text {Flox }}$ mice, this may provide a unique model system within which to study treatments for essential tremor in vivo. The authors also suggest that the increased tangles found in essential tremor patients may be the result of basket axons converging together from adjacent Purkinje neurons that may have died already (Erickson-Davis et al., 2010). Our study is the first to show that this phenomenon does occur in the cerebellum in vivo. We found that loss of Purkinje neurons resulted in the clustering of basket axons in clumps (Fig. 3.12). Interestingly, this phenotype was also found in the hippocampus, where chandelier axons, which normally target the pyramidal AIS with inhibitory GABAergic synapses, clustered and reorganized themselves in response to pyramidal cell death (Arellano et al., 2004). The conservation of this abnormal interneuron axon branching in different brain regions may represent a compensatory mechanism initiated to maintain function during neuronal dysfunction.

\subsection{Future directions for elucidating Nfasc binding partners and the mechanistic role for Nfasc in basket axon outgrowth}

The work presented here shows that Nfasc is critically required for pinceau organization and basket axon outgrowth. However, the mechanisms responsible for Nfasc 
function at the pinceau remain elusive. Future experiments should be focused on understanding the molecular signaling mechanisms within both the basket axon and Purkinje neuron during pinceau organization. First, the binding partner for Nfasc at the Purkinje AIS and/or basket terminals must be elucidated. Then the intracellular signals relayed from Nfasc interactions at the pinceau must be determined. Because it appears that basket axons in the cerebellum may function under similar mechanisms of control as chandelier cells in the cortex, understanding the mechanisms of pinceau organization may allow for a more general application to the formation of inhibitory circuit formation elsewhere in the brain (Ango et al., 2004; Huang, 2006; Huang et al., 2007; Kriebel et al., 2011; Buttermore et al., 2012). Understanding how these inhibitory interneurons are wired into neuronal circuits is critical since disruption of these circuits leads to severe neurological problems, including epilepsy, schizophrenia and autism (Denslow et al., 2001; Farber et al., 1998; Martin et al., 2000; Volk and Lewis, 2002; Wallace et al., 2001).

We assume that Nfasc functions in a heterophilic complex at the pinceau because we see different phenotypes when Nfasc is lost from only Purkinje neurons and when it is lost from both Purkinje and basket neurons. If Nfasc strictly formed a homophilic complex, then we would expect a similar phenotype in the two mutants. However, to definitely answer this question, we must obtain a basket neuron-specific Cre line that will only ablate Nfasc in basket neurons. Unfortunately, at this time no such Cre line exists. Interestingly, we did not observe defects in pinceau organization in Brevican mutant cerebella, suggesting that pinceau organization occurs normally in the absence of the ECM protein Brevican (John et al., 2006). Previous in vitro studies have shown that 
Nfasc is able to assemble a specialized ECM complex that includes Brevican at the AIS (Hedstrom et al., 2007). Brevican is clearly enriched around the Purkinje soma and AIS (Fig. 3.6) and ECM components have well-established roles in axon guidance throughout nervous system development (Moody et al., 1989; Porcionatto, 2006). It is possible that Nfasc in Brevican (Bcan) knockouts is sufficient to support pinceau formation, or that other ECM components may compensate for loss of Brevican. Additionally, other CAMs localized at the Purkinje soma and AIS, such as NrCAM, may play a role in pinceau formation as earlier studies have implicated $\mathrm{NrCAM}$ in proper circuit formation and axon guidance in vitro and in vivo (Demyanenko et al., 2011; Fitzli et al., 2000; Lustig et al., 1999). However, NrCAM mutants did not display any disruption in pinceau organization, suggesting that NrCAM does not serve as a receptor for Nfasc at the Purkinje AIS in pinceau organization. Answers to these remaining questions will come from cell specific knockout, or knock down studies that affect the functions of these proteins simultaneously.

To determine the binding partner for Nfasc, we will utilize immunoprecipitation of Nfasc from cerebellar lysate and have each of the proteins that were pulled down with Nfasc identified using Mass Spectrometry. This method requires a lot of work from a core facility with access to Mass Spectrometry and is costly, but would optimally give us a list of all the Nfasc binding partners in the cerebellum. After obtaining the list of Nfasc binding partners, immunohistochemistry of candidates would allow us to see which of these binding partners localizes to the Purkinje AIS or basket axon terminals at the pinceau. From there, the functional role of each protein in AIS and/or pinceau organization could be studied. 
To elucidate the intracellular signaling mechanism responsible for basket axon outgrowth, as regulated by Nfasc, we are already in the process of utilizing the revolutionary RNA Seq technique to compare gene expression in the cerebellum of each of our mutants, compared to wild type. RNA Seq involves the processing of RNA from tissue, which is then made into a cDNA library that can be sequenced using highthroughput sequencing machinery (Wang et al., 2009). Importantly, RNA Seq allows for quantitation of the level of expression of individual genes (Wang et al., 2009). By

extracting RNA from P10 and P20 wild type, Pcp2-Cre;Nfasc ${ }^{\text {Flox }}$, and Parv-Cre;Nfasc ${ }^{\text {Flox }}$ cerebella, we can see how the expression levels of different genes are affected by deletion of Nfasc in Purkinje and/or basket neurons. Our assumption is that the genes responsible for regulating basket axon branching will have altered expression, resulting in the abnormal branching observed in both Nfasc mutants (Fig. 3.7). Once the expression levels are compared between each of the tissues, candidate genes can be selected and immunohistochemistry for those proteins can be completed to see if protein levels are also changed in the mutants. These results will allow us to better understand the mechanisms responsible for basket axon branching and pinceau organization. Also, because Nfasc is important in nodal organization, we may discover additional novel genes that are important for the orchestration of axonal domains in myelinated axons.

\subsection{Conclusions}

The organization of myelinated axons into distinct molecular domains is critical for nervous system development and function. This organization is not only critical for the function of the neuron itself, such as at the AIS and the paranode, but also for setting 
up the signals that organize neuronal circuits, such as the importance of the Purkinje AIS in pinceau formation. Disruption of these domains and signals often leads to neuronal dysfunction and degeneration. Here, we have provided evidence for novel molecular signals responsible for the organization and stabilization of these structures. This knowledge will help us to better understand the consequences when these structures are disrupted in disease states, which may lead to more useful therapeutics for the repair or restoration of neuronal functions. 


\section{References}

Alessandri-Haber, N., Alcaraz, G., Deleuze, C., Jullien, F., Manrique, C., Couraud, F., Crest, M., and Giraud, P. (2002). Molecular determinants of emerging excitability in rat embryonic motoneurons. J. Physiol. 541, 25-39.

Alzuherri, H., and Chang, K.C. (2003). Calcineurin activates NF-kappaB in skeletal muscle C2C12 cells. Cell Signal. 15, 471-478.

Ango, F., di Cristo, G., Higashiyama, H., Bennett, V., Wu, P., and Huang, Z.J. (2004). Ankyrin-based subcellular gradient of neurofascin, an immunoglobulin family protein, directs GABAergic innervation at purkinje axon initial segment. Cell 119, 257-272.

Arellano, J.I., Munoz, A., Ballesteros-Yanez, I., Sola, R.G., and DeFelipe, J. (2004). Histopathology and reorganization of chandelier cells in the human epileptic sclerotic hippocampus. Brain 127, 45-64.

Arroyo, E.J., Sirkowski, E.E., Chitale, R., and Scherer, S.S. (2004). Acute demyelination disrupts the molecular organization of peripheral nervous system nodes. J. Comp. Neurol. $479,424-434$.

Arroyo, E.J., Xu, T., Poliak, S., Watson, M., Peles, E., and Scherer, S.S. (2001). Internodal specializations of myelinated axons in the central nervous system. Cell Tissue Res. 305, 53-66.

Baba, H., Akita, H., Ishibashi, T., Inoue, Y., Nakahira, K., and Ikenaka, K. (1999). Completion of myelin compaction, but not the attachment of oligodendroglial processes triggers $\mathrm{K}(+)$ channel clustering. J. Neurosci. Res. 58, 752-764.

Banerjee, S., Pillai, A.M., Paik, R., Li, J., and Bhat, M.A. (2006a). Axonal ensheathment and septate junction formation in the peripheral nervous system of Drosophila. J. Neurosci. 26, 3319-3329.

Banerjee, S., Sousa, A.D., and Bhat, M.A. (2006b). Organization and function of septate junctions: an evolutionary perspective. Cell. Biochem. Biophys. 46, 65-77.

Banfi, S., and Zoghbi, H.Y. (1994). Molecular genetics of hereditary ataxias. Bailliere's clinical neurology 3, 281-295.

Barger, S.W., Horster, D., Furukawa, K., Goodman, Y., Krieglstein, J., and Mattson, M.P. (1995). Tumor necrosis factors alpha and beta protect neurons against amyloid betapeptide toxicity: evidence for involvement of a kappa B-binding factor and attenuation of peroxide and Ca2+ accumulation. Proc. Natl. Acad. Sci. U. S. A. 92, 9328-9332.

Barski, J.J., Dethleffsen, K., and Meyer, M. (2000). Cre recombinase expression in cerebellar Purkinje cells. Genesis 28, 93-98. 
Bastianelli, E. (2003). Distribution of calcium-binding proteins in the cerebellum. Cerebellum 2, 242-262.

Baumgartner, S., Littleton, J.T., Broadie, K., Bhat, M.A., Harbecke, R., Lengyel, J.A., Chiquet-Ehrismann, R., Prokop, A., and Bellen, H.J. (1996). A Drosophila neurexin is required for septate junction and blood-nerve barrier formation and function. Cell 87 , 1059-1068.

Bekku, Y., Rauch, U., Ninomiya, Y., and Oohashi, T. (2009). Brevican distinctively assembles extracellular components at the large diameter nodes of Ranvier in the CNS. J. Neurochem. 108, 1266-1276.

Bellen, H.J., Lu, Y., Beckstead, R., and Bhat, M.A. (1998). Neurexin IV, caspr and paranodin--novel members of the neurexin family: encounters of axons and glia. Trends Neurosci. 21, 444-449.

Benatar, M. (2000). Neurological potassium channelopathies. QJM 93, 787-797.

Benito-Leon, J., and Louis, E.D. (2007). Clinical update: diagnosis and treatment of essential tremor. Lancet 369, 1152-1154.

Bennett, V., and Baines, A.J. (2001). Spectrin and ankyrin-based pathways: metazoan inventions for integrating cells into tissues. Physiol. Rev. 81, 1353-1392.

Bennett, V., and Lambert, S. (1999). Physiological roles of axonal ankyrins in survival of premyelinated axons and localization of voltage-gated sodium channels. J. Neurocytol. 28, 303-318.

Benton, M.D., and Raman, I.M. (2009). Stabilization of Ca current in Purkinje neurons during high-frequency firing by a balance of $\mathrm{Ca}$-dependent facilitation and inactivation. Channels 3, 393-401.

Berger, P., Niemann, A., and Suter, U. (2006). Schwann cells and the pathogenesis of inherited motor and sensory neuropathies (Charcot-Marie-Tooth disease). Glia 54, 243257.

Berghs, S., Aggujaro, D., Dirkx, R., Jr., Maksimova, E., Stabach, P., Hermel, J.M., Zhang, J.P., Philbrick, W., Slepnev, V., Ort, T., et al. (2000). betaIV spectrin, a new spectrin localized at axon initial segments and nodes of ranvier in the central and peripheral nervous system. J. Cell Biol. 151, 985-1002.

Berrebi, A.S., Oberdick, J., Sangameswaran, L., Christakos, S., Morgan, J.I., and Mugnaini, E. (1991). Cerebellar Purkinje cell markers are expressed in retinal bipolar neurons. J. Comp. Neurol. 308, 630-649. 
Bhakar, A.L., Tannis, L.L., Zeindler, C., Russo, M.P., Jobin, C., Park, D.S., MacPherson, S., and Barker, P.A. (2002). Constitutive nuclear factor-kappa B activity is required for central neuron survival. J. Neurosci. 22, 8466-8475.

Bhat, M.A. (2003). Molecular organization of axo-glial junctions. Curr. Opin. Neurobiol. $13,552-559$.

Bhat, M.A. (2004). The neurexin and NCP gene families. In Myelin Biology and Disorders, R.A. Lazarini, ed. (New York: Elsevier), pp. 579-597.

Bhat, M.A., Rios, J.C., Lu, Y., Garcia-Fresco, G.P., Ching, W., St Martin, M., Li, J., Einheber, S., Chesler, M., Rosenbluth, J., et al. (2001). Axon-glia interactions and the domain organization of myelinated axons requires neurexin IV/Caspr/Paranodin. Neuron 30, 369-383.

Biederer, T., Sara, Y., Mozhayeva, M., Atasoy, D., Liu, X., Kavalali, E.T., and Sudhof, T.C. (2002). SynCAM, a synaptic adhesion molecule that drives synapse assembly. Science 297, 1525-1531.

Black, J.A., and Waxman, S.G. (1988). The perinodal astrocyte. Glia 1, 169-183.

Bobik, M., Ellisman, M.H., Rudy, B., and Martone, M.E. (2004). Potassium channel subunit Kv3.2 and the water channel aquaporin-4 are selectively localized to cerebellar pinceau. Brain Res. 1026, 168-178.

Boiko, T., Rasband, M.N., Levinson, S.R., Caldwell, J.H., Mandel, G., Trimmer, J.S., and Matthews, G. (2001). Compact myelin dictates the differential targeting of two sodium channel isoforms in the same axon. Neuron 30, 91-104.

Boiko, T., Vakulenko, M., Ewers, H., Yap, C.C., Norden, C., and Winckler, B. (2007). Ankyrin-dependent and -independent mechanisms orchestrate axonal compartmentalization of L1 family members neurofascin and L1/neuron-glia cell adhesion molecule. J. Neurosci. 27, 590-603.

Boiko, T., Van Wart, A., Caldwell, J.H., Levinson, S.R., Trimmer, J.S., and Matthews, G. (2003). Functional specialization of the axon initial segment by isoform-specific sodium channel targeting. J. Neurosci. 23, 2306-2313.

Boyle, M.E., Berglund, E.O., Murai, K.K., Weber, L., Peles, E., and Ranscht, B. (2001). Contactin orchestrates assembly of the septate-like junctions at the paranode in myelinated peripheral nerve. Neuron 30, 385-397.

Bradke, F., and Dotti, C.G. (1999). The role of local actin instability in axon formation. Science 283, 1931-1934. 
Brakebusch, C., Seidenbecher, C.I., Asztely, F., Rauch, U., Matthies, H., Meyer, H., Krug, M., Bockers, T.M., Zhou, X., Kreutz, M.R., et al. (2002). Brevican-deficient mice display impaired hippocampal CA1 long-term potentiation but show no obvious deficits in learning and memory. Mol. Cell. Biol. 22, 7417-7427.

Brechet, A., Fache, M.P., Brachet, A., Ferracci, G., Baude, A., Irondelle, M., Pereira, S., Leterrier, C., and Dargent, B. (2008). Protein kinase CK2 contributes to the organization of sodium channels in axonal membranes by regulating their interactions with ankyrin $\mathrm{G}$. J. Cell. Biol. 183, 1101-1114.

Browne, D.L., Gancher, S.T., Nutt, J.G., Brunt, E.R., Smith, E.A., Kramer, P., and Litt, M. (1994). Episodic ataxia/myokymia syndrome is associated with point mutations in the human potassium channel gene, KCNA1. Nat. Genet. 8, 136-140.

Brownlees, J., Ackerley, S., Grierson, A.J., Jacobsen, N.J., Shea, K., Anderton, B.H., Leigh, P.N., Shaw, C.E., and Miller, C.C. (2002). Charcot-Marie-Tooth disease neurofilament mutations disrupt neurofilament assembly and axonal transport. Hum. Mol. Genet. 11, 2837-2844.

Brummendorf, T., Kenwrick, S., and Rathjen, F.G. (1998). Neural cell recognition molecule L1: from cell biology to human hereditary brain malformations. Curr. Opin. Neurobiol. 8, 87-97.

Brusco, A., Gellera, C., Cagnoli, C., Saluto, A., Castucci, A., Michielotto, C., Fetoni, V., Mariotti, C., Migone, N., Di Donato, S., et al. (2004). Molecular genetics of hereditary spinocerebellar ataxia: mutation analysis of spinocerebellar ataxia genes and CAG/CTG repeat expansion detection in 225 Italian families. Arch. Neurol. 61, 727-733.

Burkarth, N., Kriebel, M., Kranz, E.U., and Volkmer, H. (2007). Neurofascin regulates the formation of gephyrin clusters and their subsequent translocation to the axon hillock of hippocampal neurons. Mol. Cell. Neurosci. 36, 59-70.

Buttermore, E.D., Dupree, J.L., Cheng, J., An, X., Tessarollo, L., and Bhat, M.A. (2011). The Cytoskeletal Adaptor Protein Band 4.1B Is Required for the Maintenance of Paranodal Axoglial Septate Junctions in Myelinated Axons. J. Neurosci. 31, 8013-8024.

Buttermore, E.D. Piochon, C., Wallace, M.L., Philpot, B.D., Hansel, C., and Bhat, M.A. (2012). Pinceau organization in the cerebellum requires distinct functions of Neurofascin in Purkinje and basket neurons during postnatal development. J. Neurosci. In press.

Catterall, W.A. (1981). Localization of sodium channels in cultured neural cells. J. Neurosci. $1,777-783$.

Celio, M.R., Spreafico, R., De Biasi, S., and Vitellaro-Zuccarello, L. (1998). Perineuronal nets: past and present. Trends Neurosci. 21, 510-515. 
Charles, P., Tait, S., Faivre-Sarrailh, C., Barbin, G., Gunn-Moore, F., Denisenko-

Nehrbass, N., Guennoc, A.M., Girault, J.A., Brophy, P.J., and Lubetzki, C. (2002).

Neurofascin is a glial receptor for the paranodin/Caspr-contactin axonal complex at the axoglial junction. Curr. Biol. 12, 217-220.

Chen, F., Castranova, V., and Shi, X. (2001). New insights into the role of nuclear factorkappaB in cell growth regulation. Am. J. Pathol. 159, 387-397.

Chen, K., Merino, C., Sigrist, S.J., and Featherstone, D.E. (2005). The 4.1 protein coracle mediates subunit-selective anchoring of Drosophila glutamate receptors to the postsynaptic actin cytoskeleton. J. Neurosci. 25, 6667-6675.

Chen, Z., Chen, S., Chen, L., Zhou, J., Dai, Q., Yang, L., Li, X., and Zhou, L. (2009). Long-term increasing co-localization of SCN8A and ankyrin-G in rat hippocampal cornu ammonis 1 after pilocarpine induced status epilepticus. Brain Res. 1270, 112-120.

Cifuentes-Diaz, C., Chareyre, F., Garcia, M., Devaux, J., Carnaud, M., Levasseur, G., Niwa-Kawakita, M., Harroch, S., Girault, J.A., Giovannini, M., et al. (2011). Protein 4.1B contributes to the organization of peripheral myelinated axons. PLoS ONE 6, e25043.

Cobb, S.R., Buhl, E.H., Halasy, K., Paulsen, O., and Somogyi, P. (1995). Synchronization of neuronal activity in hippocampus by individual GABAergic interneurons. Nature 378, 75-78.

Coetzee, T., Fujita, N., Dupree, J., Shi, R., Blight, A., Suzuki, K., and Popko, B. (1996a). Myelination in the absence of galactocerebroside and sulfatide: normal structure with abnormal function and regional instability. Cell 86, 209-219.

Coetzee, T., Li, X., Fujita, N., Marcus, J., Suzuki, K., Francke, U., and Popko, B. (1996b). Molecular cloning, chromosomal mapping, and characterization of the mouse UDP-galactose:ceramide galactosyltransferase gene. Genomics 35, 215-222.

Collard, J.F., Cote, F., and Julien, J.P. (1995). Defective axonal transport in a transgenic mouse model of amyotrophic lateral sclerosis. Nature 375, 61-64.

Coman, I., Aigrot, M.S., Seilhean, D., Reynolds, R., Girault, J.A., Zalc, B., and Lubetzki, C. (2006). Nodal, paranodal and juxtaparanodal axonal proteins during demyelination and remyelination in multiple sclerosis. Brain 129, 3186-3195.

Court, F.A., Sherman, D.L., Pratt, T., Garry, E.M., Ribchester, R.R., Cottrell, D.F., Fleetwood-Walker, S.M., and Brophy, P.J. (2004). Restricted growth of Schwann cells lacking Cajal bands slows conduction in myelinated nerves. Nature 431, 191-195.

Craner, M.J., Newcombe, J., Black, J.A., Hartle, C., Cuzner, M.L., and Waxman, S.G. (2004). Molecular changes in neurons in multiple sclerosis: altered axonal expression of 
Nav1.2 and Nav1.6 sodium channels and $\mathrm{Na}+/ \mathrm{Ca} 2+$ exchanger. Proc. Natl. Acad. Sci. U. S. A. $101,8168-8173$.

Cruz, D.A., Weaver, C.L., Lovallo, E.M., Melchitzky, D.S., and Lewis, D.A. (2009). Selective alterations in postsynaptic markers of chandelier cell inputs to cortical pyramidal neurons in subjects with schizophrenia. Neuropsychopharmacology 34,2112 2124.

Davis, J.Q., Lambert, S., and Bennett, V. (1996). Molecular composition of the node of Ranvier: identification of ankyrin-binding cell adhesion molecules neurofascin (mucin+/third FNIII domain-) and NrCAM at nodal axon segments. J. Cell Biol. 135, 1355-1367.

Davis, T.H., Chen, C., and Isom, L.L. (2004). Sodium channel beta1 subunits promote neurite outgrowth in cerebellar granule neurons. J. Biol. Chem. 279, 51424-51432.

Demyanenko, G.P., Riday, T.T., Tran, T.S., Dalal, J., Darnell, E.P., Brennaman, L.H., Sakurai, T., Grumet, M., Philpot, B.D., and Maness, P.F. (2011). NrCAM deletion causes topographic mistargeting of thalamocortical axons to the visual cortex and disrupts visual acuity. J. Neurosci. 31, 1545-1558.

Deng, H., Le, W., and Jankovic, J. (2007). Genetics of essential tremor. Brain 130, 14561464.

Deng, J., Xia, W., Miller, S.A., Wen, Y., Wang, H.Y., and Hung, M.C. (2004).

Crossregulation of NF-kappaB by the APC/GSK-3beta/beta-catenin pathway. Mol.

Carcinog. 39, 139-146.

Denisenko-Nehrbass, N., Oguievetskaia, K., Goutebroze, L., Galvez, T., Yamakawa, H., Ohara, O., Carnaud, M., and Girault, J.A. (2003). Protein 4.1B associates with both Caspr/paranodin and Caspr2 at paranodes and juxtaparanodes of myelinated fibres. Eur. J. Neurosci. 17, 411-416.

Denslow, M.J., Eid, T., Du, F., Schwarcz, R., Lothman, E.W., and Steward, O. (2001). Disruption of inhibition in area CA1 of the hippocampus in a rat model of temporal lobe epilepsy. J. Neurophysiol. 86, 2231-2245.

Dodd, J., Morton, S.B., Karagogeos, D., Yamamoto, M., and Jessell, T.M. (1988). Spatial regulation of axonal glycoprotein expression on subsets of embryonic spinal neurons. Neuron 1, 105-116.

Doherty, P., Williams, G., and Williams, E.J. (2000). CAMs and axonal growth: a critical evaluation of the role of calcium and the MAPK cascade. Mol. Cell. Neurosci. 16, 283295. 
Dupree, J.L., Girault, J.A., and Popko, B. (1999). Axo-glial interactions regulate the localization of axonal paranodal proteins. J. Cell Biol. 147, 1145-1152.

Dupree, J.L., and Popko, B. (1999). Genetic dissection of myelin galactolipid function. J. Neurocytol. 28, 271-279.

Dzhashiashvili, Y., Zhang, Y., Galinska, J., Lam, I., Grumet, M., and Salzer, J.L. (2007). Nodes of Ranvier and axon initial segments are ankyrin G-dependent domains that assemble by distinct mechanisms. J. Cell Biol. 177, 857-870.

Einheber, S., Bhat, M.A., and Salzer, J.L. (2006). Disrupted Axo-Glial Junctions Result in Accumulation of Abnormal Mitochondria at Nodes of Ranvier. Neuron Glia Biol. 2, 165-174.

Einheber, S., Zanazzi, G., Ching, W., Scherer, S., Milner, T.A., Peles, E., and Salzer, J.L. (1997). The axonal membrane protein Caspr, a homologue of neurexin IV, is a component of the septate-like paranodal junctions that assemble during myelination. J. Cell Biol. 139, 1495-1506.

Elder, G.A., Friedrich, V.L., Jr., and Lazzarini, R.A. (2001). Schwann cells and oligodendrocytes read distinct signals in establishing myelin sheath thickness. J. Neurosci. Res. 65, 493-499.

Erickson-Davis, C.R., Faust, P.L., Vonsattel, J.P., Gupta, S., Honig, L.S., and Louis, E.D. (2010). "Hairy baskets" associated with degenerative Purkinje cell changes in essential tremor. J. Neuropathol. Exp. Neurol. 69, 262-271.

Eshed, Y., Feinberg, K., Carey, D.J., and Peles, E. (2007). Secreted gliomedin is a perinodal matrix component of peripheral nerves. J. Cell Biol. 177, 551-562.

Eshed, Y., Feinberg, K., Poliak, S., Sabanay, H., Sarig-Nadir, O., Spiegel, I., Bermingham, J.R., Jr., and Peles, E. (2005). Gliomedin mediates Schwann cell-axon interaction and the molecular assembly of the nodes of Ranvier. Neuron 47, 215-229.

Fabrizi, G.M., Cavallaro, T., Angiari, C., Cabrini, I., Taioli, F., Malerba, G., Bertolasi, L., and Rizzuto, N. (2007). Charcot-Marie-Tooth disease type 2E, a disorder of the cytoskeleton. Brain 130, 394-403.

Faivre-Sarrailh, C., Banerjee, S., Li, J., Hortsch, M., Laval, M., and Bhat, M.A. (2004). Drosophila contactin, a homolog of vertebrate contactin, is required for septate junction organization and paracellular barrier function. Development 131, 4931-4942.

Faivre-Sarrailh, C., Gauthier, F., Denisenko-Nehrbass, N., Le Bivic, A., Rougon, G., and Girault, J.A. (2000). The glycosylphosphatidyl inositol-anchored adhesion molecule $\mathrm{F} 3 /$ contactin is required for surface transport of paranodin/contactin-associated protein (caspr). J. Cell Biol. 149, 491-502. 
Farber, N.B., Newcomer, J.W., and Olney, J.W. (1998). The glutamate synapse in neuropsychiatric disorders. Focus on schizophrenia and Alzheimer's disease. Prog. Brain Res. 116, 421-437.

Fehon, R.G., Dawson, I.A., and Artavanis-Tsakonas, S. (1994). A Drosophila homologue of membrane-skeleton protein 4.1 is associated with septate junctions and is encoded by the coracle gene. Development 120, 545-557.

Feinberg, K., Eshed-Eisenbach, Y., Frechter, S., Amor, V., Salomon, D., Sabanay, H., Dupree, J.L., Grumet, M., Brophy, P.J., Shrager, P., et al. (2010). A glial signal consisting of gliomedin and NrCAM clusters axonal $\mathrm{Na}+$ channels during the formation of nodes of Ranvier. Neuron 65, 490-502.

Fitzli, D., Stoeckli, E.T., Kunz, S., Siribour, K., Rader, C., Kunz, B., Kozlov, S.V., Buchstaller, A., Lane, R.P., Suter, D.M., et al. (2000). A direct interaction of axonin-1 with $\mathrm{NgCAM}$-related cell adhesion molecule (NrCAM) results in guidance, but not growth of commissural axons. J. Cell Biol. 149, 951-968.

Freund, T.F., and Buzsaki, G. (1996). Interneurons of the hippocampus. Hippocampus 6 , 347-470.

Fried, S.I., Lasker, A.C., Desai, N.J., Eddington, D.K., and Rizzo, J.F., 3rd (2009). Axonal sodium-channel bands shape the response to electric stimulation in retinal ganglion cells. J. Neurophysiol. 101, 1972-1987.

Fukata, Y., Itoh, T.J., Kimura, T., Menager, C., Nishimura, T., Shiromizu, T., Watanabe, H., Inagaki, N., Iwamatsu, A., Hotani, H., et al. (2002a). CRMP-2 binds to tubulin heterodimers to promote microtubule assembly. Nat. Cell Biol. 4, 583-591.

Fukata, Y., Kimura, T., and Kaibuchi, K. (2002b). Axon specification in hippocampal neurons. Neurosci. Res. 43, 305-315.

Garcia-Fresco, G.P., Sousa, A.D., Pillai, A.M., Moy, S.S., Crawley, J.N., Tessarollo, L., Dupree, J.L., and Bhat, M.A. (2006). Disruption of axo-glial junctions causes cytoskeletal disorganization and degeneration of Purkinje neuron axons. Proc. Natl. Acad. Sci. U. S. A. 103, 5137-5142.

Gardner-Medwin, A.R. (1983). Analysis of potassium dynamics in mammalian brain tissue. J. Physiol. 335, 393-426.

Gardner-Medwin, A.R. (1986). A new framework for assessment of potassium-buffering mechanisms. Ann. N. Y. Acad. Sci. 481, 287-302.

Garrido, J.J., Giraud, P., Carlier, E., Fernandes, F., Moussif, A., Fache, M.P., Debanne, D., and Dargent, B. (2003). A targeting motif involved in sodium channel clustering at the axonal initial segment. Science 300, 2091-2094. 
Garver, T.D., Ren, Q., Tuvia, S., and Bennett, V. (1997). Tyrosine phosphorylation at a site highly conserved in the L1 family of cell adhesion molecules abolishes ankyrin binding and increases lateral mobility of neurofascin. J. Cell Biol. 137, 703-714.

Ghabriel, M.N., and Allt, G. (1981). Incisures of Schmidt-Lanterman. Prog. Neurobiol. $17,25-58$.

Girault, J.A., Labesse, G., Mornon, J.P., and Callebaut, I. (1998). Janus kinases and focal adhesion kinases play in the 4.1 band: a superfamily of band 4.1 domains important for cell structure and signal transduction. Mol. Med. 4, 751-769.

Gobel, S. (1971). Axo-axonic septate junctions in the basket formations of the cat cerebellar cortex. J. Cell Biol. 51, 328-333.

Gold, D.A., Baek, S.H., Schork, N.J., Rose, D.W., Larsen, D.D., Sachs, B.D., Rosenfeld, M.G., and Hamilton, B.A. (2003). RORalpha coordinates reciprocal signaling in cerebellar development through sonic hedgehog and calcium-dependent pathways. Neuron 40, 1119-1131.

Gollan, L., Sabanay, H., Poliak, S., Berglund, E.O., Ranscht, B., and Peles, E. (2002). Retention of a cell adhesion complex at the paranodal junction requires the cytoplasmic region of Caspr. J. Cell Biol. 157, 1247-1256.

Grubb, M.S., and Burrone, J. (2010a). Activity-dependent relocation of the axon initial segment fine-tunes neuronal excitability. Nature 465, 1070-1074.

Grubb, M.S., and Burrone, J. (2010b). Building and maintaining the axon initial segment. Curr. Opin. Neurobiol. 20, 481-488.

Grubb, M.S., Shu, Y., Kuba, H., Rasband, M.N., Wimmer, V.C., and Bender, K.J. (2011). Short- and long-term plasticity at the axon initial segment. J. Neurosci. 31, 16049-16055.

Grumet, M. (1997). Nr-CAM: a cell adhesion molecule with ligand and receptor functions. Cell Tissue Res. 290, 423-428.

Hacker, H., and Karin, M. (2006). Regulation and function of IKK and IKK-related kinases. Sci. STKE 2006, re13.

Hamilton, B.A., Frankel, W.N., Kerrebrock, A.W., Hawkins, T.L., FitzHugh, W., Kusumi, K., Russell, L.B., Mueller, K.L., van Berkel, V., Birren, B.W., et al. (1996). Disruption of the nuclear hormone receptor RORalpha in staggerer mice. Nature 379, 736-739. 
Hansen, S.M., Berezin, V., and Bock, E. (2008). Signaling mechanisms of neurite outgrowth induced by the cell adhesion molecules NCAM and N-cadherin. Cell Mol. Life Sci. 65, 3809-3821.

Harding, A.E. (1993). Clinical features and classification of inherited ataxias. Adv. Neurol. 61, 1-14.

Hartline, D.K. (2008). What is myelin? Neuron Glia Biol. 4, 153-163.

Hedstrom, K.L., Ogawa, Y., and Rasband, M.N. (2008). AnkyrinG is required for maintenance of the axon initial segment and neuronal polarity. J. Cell Biol. 183, 635-640.

Hedstrom, K.L., and Rasband, M.N. (2006). Intrinsic and extrinsic determinants of ion channel localization in neurons. J. Neurochem. 98, 1345-1352.

Hedstrom, K.L., Xu, X., Ogawa, Y., Frischknecht, R., Seidenbecher, C.I., Shrager, P., and Rasband, M.N. (2007). Neurofascin assembles a specialized extracellular matrix at the axon initial segment. J. Cell Biol. 178, 875-886.

Hildebrand, C., Remahl, S., Persson, H., and Bjartmar, C. (1993). Myelinated nerve fibres in the CNS. Prog. Neurobiol. 40, 319-384.

Hill, A.S., Nishino, A., Nakajo, K., Zhang, G., Fineman, J.R., Selzer, M.E., Okamura, Y., and Cooper, E.C. (2008). Ion channel clustering at the axon initial segment and node of Ranvier evolved sequentially in early chordates. PLoS Genet. 4, e1000317.

Hille, B. (2001). Ion Channels of Excitable Membranes. (Sinauer Associates, Sunderland, Massachusettes.)

Hippenmeyer, S., Vrieseling, E., Sigrist, M., Portmann, T., Laengle, C., Ladle, D.R., and Arber, S. (2005). A developmental switch in the response of DRG neurons to ETS transcription factor signaling. PLoS Biol. 3, e159.

Honke, K., Hirahara, Y., Dupree, J., Suzuki, K., Popko, B., Fukushima, K., Fukushima, J., Nagasawa, T., Yoshida, N., Wada, Y., et al. (2002). Paranodal junction formation and spermatogenesis require sulfoglycolipids. Proc. Natl. Acad. Sci. U. S. A. 99, 4227-4232.

Hoover, K.B., and Bryant, P.J. (2000). The genetics of the protein 4.1 family: organizers of the membrane and cytoskeleton. Curr. Opin. Cell Biol. 12, 229-234.

Horresh, I., Bar, V., Kissil, J.L., and Peles, E. (2010). Organization of myelinated axons by Caspr and Caspr2 requires the cytoskeletal adapter protein 4.1B. J. Neurosci. 30, 2480-2489. 
Horresh, I., Poliak, S., Grant, S., Bredt, D., Rasband, M.N., and Peles, E. (2008). Multiple molecular interactions determine the clustering of Caspr 2 and Kv1 channels in myelinated axons. J. Neurosci. 28, 14213-14222.

Howell, O.W., Palser, A., Polito, A., Melrose, S., Zonta, B., Scheiermann, C., Vora, A.J., Brophy, P.J., and Reynolds, R. (2006). Disruption of neurofascin localization reveals early changes preceding demyelination and remyelination in multiple sclerosis. Brain $129,3173-3185$.

Hu, W., Tian, C., Li, T., Yang, M., Hou, H., and Shu, Y. (2009). Distinct contributions of $\mathrm{Na}(\mathrm{v}) 1.6$ and $\mathrm{Na}(\mathrm{v}) 1.2$ in action potential initiation and backpropagation. Nat. Neurosci. $12,996-1002$.

Huang, Z.J. (2006). Subcellular organization of GABAergic synapses: role of ankyrins and L1 cell adhesion molecules. Nat. Neurosci. 9, 163-166.

Huang, Z.J., Di Cristo, G., and Ango, F. (2007). Development of GABA innervation in the cerebral and cerebellar cortices. Nat. Rev. Neurosci. 8, 673-686.

Huxley, A.F., and Stampfli, R. (1949). Evidence for saltatory conduction in peripheral myelinated nerve fibres. J. Physiol. 108, 315-339.

Inagaki, N., Chihara, K., Arimura, N., Menager, C., Kawano, Y., Matsuo, N., Nishimura, T., Amano, M., and Kaibuchi, K. (2001). CRMP-2 induces axons in cultured hippocampal neurons. Nat. Neurosci. 4, 781-782.

Ishibashi, T., Dupree, J.L., Ikenaka, K., Hirahara, Y., Honke, K., Peles, E., Popko, B., Suzuki, K., Nishino, H., and Baba, H. (2002). A myelin galactolipid, sulfatide, is essential for maintenance of ion channels on myelinated ax on but not essential for initial cluster formation. J. Neurosci. 22, 6507-6514.

Isom, L.L. (2002). The role of sodium channels in cell adhesion. Front. Biosci. 7, 12-23.

Ivanovic, A., Horresh, I., Golan, N., Spiegel, I., Sabanay, H., Frechter, S., Ohno, S., Terada, N., Mobius, W., Rosenbluth, J., et al. (2012). The cytoskeletal adapter protein 4.1G organizes the internodes in peripheral myelinated nerves. J. Cell Biol. 196, 337-344.

Jeffress, L.A. (1948). A place theory of sound localization. J. Comp. Physiol. Psychol. $41,35-39$.

Jekely, G. (2007). Eukaryotic membranes and cytoskeleton, Vol 607 (New York: Springer).

Jenkins, S.M., and Bennett, V. (2001). Ankyrin-G coordinates assembly of the spectrinbased membrane skeleton, voltage-gated sodium channels, and L1 CAMs at Purkinje neuron initial segments. J. Cell Biol. 155, 739-746. 
Jessen, K.R., and Mirsky, R. (2005). The origin and development of glial cells in peripheral nerves. Nat. Rev. Neurosci. 6, 671-682.

John, N., Krugel, H., Frischknecht, R., Smalla, K.H., Schultz, C., Kreutz, M.R., Gundelfinger, E.D., and Seidenbecher, C.I. (2006). Brevican-containing perineuronal nets of extracellular matrix in dissociated hippocampal primary cultures. Mol. Cell. Neurosci. $31,774-784$.

Johnston, J., Griffin, S.J., Baker, C., Skrzypiec, A., Chernova, T., and Forsythe, I.D. (2008). Initial segment Kv2.2 channels mediate a slow delayed rectifier and maintain high frequency action potential firing in medial nucleus of the trapezoid body neurons. J. Physiol. 586, 3493-3509.

Kaltschmidt, B., Sparna, T., and Kaltschmidt, C. (1999a). Activation of NF-kappa B by reactive oxygen intermediates in the nervous system. Antioxid. Redox Signal. 1, 129-144.

Kaltschmidt, B., Uherek, M., Wellmann, H., Volk, B., and Kaltschmidt, C. (1999b). Inhibition of NF-kappaB potentiates amyloid beta-mediated neuronal apoptosis. Proc. Natl. Acad. Sci. U. S. A. 96, 9409-9414.

Kang, Q., Wang, T., Zhang, H., Mohandas, N., and An, X. (2009). A Golgi-associated protein $4.1 \mathrm{~B}$ variant is required for assimilation of proteins in the membrane. J. Cell Sci. 122, 1091-1099.

Kaphzan, H., Buffington, S.A., Jung, J.I., Rasband, M.N., and Klann, E. (2011). Alterations in intrinsic membrane properties and the axon initial segment in a mouse model of Angelman syndrome. J. Neurosci. 31, 17637-17648.

Kaplan, M.R., Cho, M.H., Ullian, E.M., Isom, L.L., Levinson, S.R., and Barres, B.A. (2001). Differential control of clustering of the sodium channels $\mathrm{Na}(\mathrm{v}) 1.2$ and $\mathrm{Na}(\mathrm{v}) 1.6$ at developing CNS nodes of Ranvier. Neuron 30, 105-119.

Khaliq, Z.M., Gouwens, N.W., and Raman, I.M. (2003). The contribution of resurgent sodium current to high-frequency firing in Purkinje neurons: an experimental and modeling study. J. Neurosci. 23, 4899-4912.

Khaliq, Z.M., and Raman, I.M. (2006). Relative contributions of axonal and somatic Na channels to action potential initiation in cerebellar Purkinje neurons. J. Neurosci. 26, 1935-1944.

Kimura, T., Watanabe, H., Iwamatsu, A., and Kaibuchi, K. (2005). Tubulin and CRMP-2 complex is transported via Kinesin-1. J. Neurochem. 93, 1371-1382.

Kirschbaum, K., Kriebel, M., Kranz, E.U., Potz, O., and Volkmer, H. (2009). Analysis of non-canonical fibroblast growth factor receptor 1 (FGFR1) interaction reveals regulatory and activating domains of neurofascin. J. Biol. Chem. 284, 28533-28542. 
Klugmann, M., Schwab, M.H., Puhlhofer, A., Schneider, A., Zimmermann, F., Griffiths, I.R., and Nave, K.A. (1997). Assembly of CNS myelin in the absence of proteolipid protein. Neuron 18, 59-70.

Kole, M.H., Ilschner, S.U., Kampa, B.M., Williams, S.R., Ruben, P.C., and Stuart, G.J. (2008). Action potential generation requires a high sodium channel density in the axon initial segment. Nat. Neurosci. 11, 178-186.

Kolodkin, A.L., and Tessier-Lavigne, M. (2011). Mechanisms and molecules of neuronal wiring: a primer. Cold Spring Harb. Perspect. Biol. 3.

Komada, M., and Soriano, P. (2002). [Beta]IV-spectrin regulates sodium channel clustering through ankyrin-G at axon initial segments and nodes of Ranvier. J. Cell Biol. $156,337-348$.

Konishi, M. (2003). Coding of auditory space. Annu. Rev. Neurosci. 26, 31-55.

Korn, H., and Axelrad, H. (1980). Electrical inhibition of Purkinje cells in the cerebellum of the rat. Proc. Natl. Acad. Sci. U. S. A. 77, 6244-6247.

Koster, B., Deuschl, G., Lauk, M., Timmer, J., Guschlbauer, B., and Lucking, C.H. (2002). Essential tremor and cerebellar dysfunction: abnormal ballistic movements. J. Neurol. Neurosurg. Psychiatry 73, 400-405.

Koticha, D., Babiarz, J., Kane-Goldsmith, N., Jacob, J., Raju, K., and Grumet, M. (2005). Cell adhesion and neurite outgrowth are promoted by neurofascin NF155 and inhibited by NF186. Mol. Cell. Neurosci. 30, 137-148.

Koticha, D., Maurel, P., Zanazzi, G., Kane-Goldsmith, N., Basak, S., Babiarz, J., Salzer, J., and Grumet, M. (2006). Neurofascin interactions play a critical role in clustering sodium channels, ankyrin G and beta IV spectrin at peripheral nodes of Ranvier. Dev. Biol. 293, 1-12.

Kriebel, M., Metzger, J., Trinks, S., Chugh, D., Harvey, R.J., Harvey, K., and Volkmer, H. (2011). The Cell Adhesion Molecule Neurofascin Stabilizes Axo-axonic GABAergic Terminals at the Axon Initial Segment. J. Biol. Chem. 286, 24385-24393.

Kuba, H., Ishii, T.M., and Ohmori, H. (2006). Axonal site of spike initiation enhances auditory coincidence detection. Nature 444, 1069-1072.

Kuba, H., Oichi, Y., and Ohmori, H. (2010). Presynaptic activity regulates $\mathrm{Na}(+)$ channel distribution at the axon initial segment. Nature 465, 1075-1078.

Labasque, M., Devaux, J.J., Leveque, C., and Faivre-Sarrailh, C. (2011). Fibronectin type III-like domains of neurofascin-186 protein mediate gliomedin binding and its clustering at the developing nodes of Ranvier. J. Biol. Chem. 286, 42426-42434. 
Lacas-Gervais, S., Guo, J., Strenzke, N., Scarfone, E., Kolpe, M., Jahkel, M., De Camilli, P., Moser, T., Rasband, M.N., and Solimena, M. (2004). BetaIVSigma1 spectrin stabilizes the nodes of Ranvier and axon initial segments. J. Cell Biol. 166, 983-990.

Lai, C., Brow, M.A., Nave, K.A., Noronha, A.B., Quarles, R.H., Bloom, F.E., Milner, R.J., and Sutcliffe, J.G. (1987). Two forms of 1B236/myelin-associated glycoprotein, a cell adhesion molecule for postnatal neural development, are produced by alternative splicing. Proc. Natl. Acad. Sci. U. S. A. 84, 4337-4341.

Lamb, R.S., Ward, R.E., Schweizer, L., and Fehon, R.G. (1998). Drosophila coracle, a member of the protein 4.1 superfamily, has essential structural functions in the septate junctions and developmental functions in embryonic and adult epithelial cells. Mol. Biol. Cell 9, 3505-3519.

Lambert, S., Davis, J.Q., and Bennett, V. (1997). Morphogenesis of the node of Ranvier: co-clusters of ankyrin and ankyrin-binding integral proteins define early developmental intermediates. J. Neurosci. 17, 7025-7036.

Lappe-Siefke, C., Goebbels, S., Gravel, M., Nicksch, E., Lee, J., Braun, P.E., Griffiths, I.R., and Nave, K.A. (2003). Disruption of Cnp1 uncouples oligodendroglial functions in axonal support and myelination. Nat. Genet. 33, 366-374.

Laube, G., Roper, J., Pitt, J.C., Sewing, S., Kistner, U., Garner, C.C., Pongs, O., and Veh, R.W. (1996). Ultrastructural localization of Shaker-related potassium channel subunits and synapse-associated protein 90 to septate-like junctions in rat cerebellar Pinceaux. Brain Res. Mol. Brain Res. 42, 51-61.

Lemaillet, G., Walker, B., and Lambert, S. (2003). Identification of a conserved ankyrinbinding motif in the family of sodium channel alpha subunits. J. Biol. Chem. 278, 2733327339.

Levin, S.I., Khaliq, Z.M., Aman, T.K., Grieco, T.M., Kearney, J.A., Raman, I.M., and Meisler, M.H. (2006). Impaired motor function in mice with cell-specific knockout of sodium channel Scn8a (NaV1.6) in cerebellar purkinje neurons and granule cells. J. Neurophysiol. 96, 785-793.

Lewis, D.A., Hashimoto, T., and Volk, D.W. (2005). Cortical inhibitory neurons and schizophrenia. Nat. Rev. Neurosci. 6, 312-324.

Li, C., Tropak, M.B., Gerlai, R., Clapoff, S., Abramow-Newerly, W., Trapp, B., Peterson, A., and Roder, J. (1994). Myelination in the absence of myelin-associated glycoprotein. Nature 369, 747-750.

Li, X.G., Somogyi, P., Tepper, J.M., and Buzsaki, G. (1992). Axonal and dendritic arborization of an intracellularly labeled chandelier cell in the CA1 region of rat hippocampus. Exp. Brain Res. 90, 519-525. 
Lonigro, A., and Devaux, J.J. (2009). Disruption of neurofascin and gliomedin at nodes of Ranvier precedes demyelination in experimental allergic neuritis. Brain 132, 260-273.

Lopantsev, V., Tempel, B.L., and Schwartzkroin, P.A. (2003). Hyperexcitability of CA3 pyramidal cells in mice lacking the potassium channel subunit Kv1.1. Epilepsia 44, 15061512.

Lorincz, A., and Nusser, Z. (2008). Cell-type-dependent molecular composition of the axon initial segment. J. Neurosci. 28, 14329-14340.

Louis, E.D. (2001). Etiology of essential tremor: should we be searching for environmental causes? Mov. Disord. 16, 822-829.

Louis, E.D., Faust, P.L., Vonsattel, J.P., Honig, L.S., Rajput, A., Robinson, C.A., Rajput, A., Pahwa, R., Lyons, K.E., Ross, G.W., et al. (2007). Neuropathological changes in essential tremor: 33 cases compared with 21 controls. Brain 130, 3297-3307.

Louis, E.D., Yi, H., Erickson-Davis, C., Vonsattel, J.P., and Faust, P.L. (2009). Structural study of Purkinje cell axonal torpedoes in essential tremor. Neurosci. Lett. 450, 287-291.

Lu, B., Wang, K.H., and Nose, A. (2009). Molecular mechanisms underlying neural circuit formation. Curr. Opin. Neurobiol. 19, 162-167.

Lubetzki, C., Williams, A., and Stankoff, B. (2005). Promoting repair in multiple sclerosis: problems and prospects. Curr. Opin. Neurol. 18, 237-244.

Lustig, M., Sakurai, T., and Grumet, M. (1999). Nr-CAM promotes neurite outgrowth from peripheral ganglia by a mechanism involving axonin-1 as a neuronal receptor. Dev. Biol. 209, 340-351.

Lustig, M., Zanazzi, G., Sakurai, T., Blanco, C., Levinson, S.R., Lambert, S., Grumet, M., and Salzer, J.L. (2001). Nr-CAM and neurofascin interactions regulate ankyrin G and sodium channel clustering at the node of Ranvier. Curr. Biol. 11, 1864-1869.

Maier, O., Baron, W., and Hoekstra, D. (2007). Reduced raft-association of NF155 in active MS-lesions is accompanied by the disruption of the paranodal junction. Glia 55, 885-895.

Maier, O., van der Heide, T., van Dam, A.M., Baron, W., de Vries, H., and Hoekstra, D. (2005). Alteration of the extracellular matrix interferes with raft association of neurofascin in oligodendrocytes. Potential significance for multiple sclerosis? Mol. Cell. Neurosci. 28, 390-401.

Malhotra, J.D., Kazen-Gillespie, K., Hortsch, M., and Isom, L.L. (2000). Sodium channel beta subunits mediate homophilic cell adhesion and recruit ankyrin to points of cell-cell contact. J. Biol. Chem. 275, 11383-11388. 
Maniar, T.A., Kaplan, M., Wang, G.J., Shen, K., Wei, L., Shaw, J.E., Koushika, S.P., and Bargmann, C.I. (2012). UNC-33 (CRMP) and ankyrin organize microtubules and localize kinesin to polarize axon-dendrite sorting. Nat. Neurosci. 15, 48-56.

Manto, M., and Marmolino, D. (2009). Cerebellar ataxias. Curr. Opin. Neurol. 22, 419429.

Marcus, J., Dupree, J.L., and Popko, B. (2002). Myelin-associated glycoprotein and myelin galactolipids stabilize developing axo-glial interactions. J. Cell Biol. 156, 567577.

Marcus, J., Honigbaum, S., Shroff, S., Honke, K., Rosenbluth, J., and Dupree, J.L. (2006). Sulfatide is essential for the maintenance of CNS myelin and axon structure. Glia $53,372-381$.

Mark, M.D., Lohrum, M., and Puschel, A.W. (1997). Patterning neuronal connections by chemorepulsion: the semaphorins. Cell Tissue Res. 290, 299-306.

Martin, E.R., Menold, M.M., Wolpert, C.M., Bass, M.P., Donnelly, S.L., Ravan, S.A., Zimmerman, A., Gilbert, J.R., Vance, J.M., Maddox, L.O., et al. (2000). Analysis of linkage disequilibrium in gamma-aminobutyric acid receptor subunit genes in autistic disorder. Am. J. Med. Genet. 96, 43-48.

Masuda, M., Yageta, M., Fukuhara, H., Kuramochi, M., Maruyama, T., Nomoto, A., and Murakami, Y. (2002). The tumor suppressor protein TSLC1 is involved in cell-cell adhesion. J. Biol. Chem. 277, 31014-31019.

Mathey, E.K., Derfuss, T., Storch, M.K., Williams, K.R., Hales, K., Woolley, D.R., AlHayani, A., Davies, S.N., Rasband, M.N., Olsson, T., et al. (2007). Neurofascin as a novel target for autoantibody-mediated axonal injury. J. Exp. Med. 204, 2363-2372.

Maurel, P., Einheber, S., Galinska, J., Thaker, P., Lam, I., Rubin, M.B., Scherer, S.S., Murakami, Y., Gutmann, D.H., and Salzer, J.L. (2007). Nectin-like proteins mediate axon Schwann cell interactions along the internode and are essential for myelination. J. Cell Biol. 178, 861-874.

Mburu, P., Kikkawa, Y., Townsend, S., Romero, R., Yonekawa, H., and Brown, S.D. (2006). Whirlin complexes with p55 at the stereocilia tip during hair cell development. Proc. Natl. Acad. Sci. U. S. A. 103, 10973-10978.

McEwen, D.P., and Isom, L.L. (2004). Heterophilic interactions of sodium channel beta1 subunits with axonal and glial cell adhesion molecules. J. Biol. Chem. 279, 52744-52752.

Meadows, L.S., Malhotra, J., Loukas, A., Thyagarajan, V., Kazen-Gillespie, K.A., Koopman, M.C., Kriegler, S., Isom, L.L., and Ragsdale, D.S. (2002). Functional and 
biochemical analysis of a sodium channel beta1 subunit mutation responsible for generalized epilepsy with febrile seizures plus type 1. J. Neurosci. 22, 10699-10709.

Melendez-Vasquez, C., Carey, D.J., Zanazzi, G., Reizes, O., Maurel, P., and Salzer, J.L. (2005). Differential expression of proteoglycans at central and peripheral nodes of Ranvier. Glia 52, 301-308.

Menegoz, M., Gaspar, P., Le Bert, M., Galvez, T., Burgaya, F., Palfrey, C., Ezan, P., Arnos, F., and Girault, J.A. (1997). Paranodin, a glycoprotein of neuronal paranodal membranes. Neuron 19, 319-331.

Mercurio, F., Zhu, H., Murray, B.W., Shevchenko, A., Bennett, B.L., Li, J., Young, D.B., Barbosa, M., Mann, M., Manning, A., et al. (1997). IKK-1 and IKK-2: cytokine-activated IkappaB kinases essential for NF-kappaB activation. Science 278, 860-866.

Meyrand, P., Weimann, J.M., and Marder, E. (1992). Multiple axonal spike initiation zones in a motor neuron: serotonin activation. J. Neurosci. 12, 2803-2812.

Michailov, G.V., Sereda, M.W., Brinkmann, B.G., Fischer, T.M., Haug, B., Birchmeier, C., Role, L., Lai, C., Schwab, M.H., and Nave, K.A. (2004). Axonal neuregulin-1 regulates myelin sheath thickness. Science 304, 700-703.

Montag, D., Giese, K.P., Bartsch, U., Martini, R., Lang, Y., Bluthmann, H., Karthigasan, J., Kirschner, D.A., Wintergerst, E.S., Nave, K.A., et al. (1994). Mice deficient for the myelin-associated glycoprotein show subtle abnormalities in myelin. Neuron 13, 229246.

Moody, S.A., Quigg, M.S., and Little, C.D. (1989). Extracellular matrix components of the peripheral pathway of chick trigeminal axons. J. Comp. Neurol. 283, 38-53.

Nakada, C., Ritchie, K., Oba, Y., Nakamura, M., Hotta, Y., Iino, R., Kasai, R.S., Yamaguchi, K., Fujiwara, T., and Kusumi, A. (2003). Accumulation of anchored proteins forms membrane diffusion barriers during neuronal polarization. Nat. Cell Biol. 5, 626632.

Nave, K.A., Sereda, M.W., and Ehrenreich, H. (2007). Mechanisms of disease: inherited demyelinating neuropathies--from basic to clinical research. Nat. Clin. Pract. Neurol. 3, 453-464.

Nordquist, D.T., Kozak, C.A., and Orr, H.T. (1988). cDNA cloning and characterization of three genes uniquely expressed in cerebellum by Purkinje neurons. J. Neurosci. 8 , 4780-4789.

Ogawa, Y., Horresh, I., Trimmer, J.S., Bredt, D.S., Peles, E., and Rasband, M.N. (2008). Postsynaptic density- 93 clusters Kv1 channels at axon initial segments independently of Caspr2. J. Neurosci. 28, 5731-5739. 
Ogawa, Y., Schafer, D.P., Horresh, I., Bar, V., Hales, K., Yang, Y., Susuki, K., Peles, E., Stankewich, M.C., and Rasband, M.N. (2006). Spectrins and ankyrinB constitute a specialized paranodal cytoskeleton. J. Neurosci. 26, 5230-5239.

Ogita, H., and Takai, Y. (2006). Nectins and nectin-like molecules: roles in cell adhesion, polarization, movement, and proliferation. IUBMB life 58, 334-343.

Oguievetskaia, K., Cifuentes-Diaz, C., Girault, J.A., and Goutebroze, L. (2005). Cellular contacts in myelinated fibers of the peripheral nervous system. Med. Sci. (Paris) 21, 162169.

Ohara, R., Yamakawa, H., Nakayama, M., and Ohara, O. (2000). Type II brain 4.1 (4.1B/KIAA0987), a member of the protein 4.1 family, is localized to neuronal paranodes. Brain. Res. Mol. Brain. Res. 85, 41-52.

Okumura, K., Mochizuki, E., Yokohama, M., Yamakawa, H., Shitara, H., Mburu, P., Yonekawa, H., Brown, S.D., and Kikkawa, Y. (2010). Protein 4.1 expression in the developing hair cells of the mouse inner ear. Brain. Res. 1307, 53-62.

Ophoff, R.A., Terwindt, G.M., Vergouwe, M.N., van Eijk, R., Oefner, P.J., Hoffman, S.M., Lamerdin, J.E., Mohrenweiser, H.W., Bulman, D.E., Ferrari, M., et al. (1996). Familial hemiplegic migraine and episodic ataxia type- 2 are caused by mutations in the Ca2+ channel gene CACNL1A4. Cell 87, 543-552.

Palay, S.L., and Palay, V.C. (1974). Cerebellar Cortex (New York: Springer-Verlag).

Pan, Z., Kao, T., Horvath, Z., Lemos, J., Sul, J.Y., Cranstoun, S.D., Bennett, V., Scherer, S.S., and Cooper, E.C. (2006). A common ankyrin-G-based mechanism retains KCNQ and $\mathrm{NaV}$ channels at electrically active domains of the axon. J. Neurosci. 26, 2599-2613.

Parisi, S.L., Heroux, M.E., Culham, E.G., and Norman, K.E. (2006). Functional mobility and postural control in essential tremor. Arch. Phys. Med. Rehabil. 87, 1357-1364.

Parra, M., Gascard, P., Walensky, L.D., Gimm, J.A., Blackshaw, S., Chan, N., Takakuwa, Y., Berger, T., Lee, G., Chasis, J.A., et al. (2000). Molecular and functional characterization of protein 4.1B, a novel member of the protein 4.1 family with high level, focal expression in brain. J. Biol. Chem. 275, 3247-3255.

Pasterkamp, R.J., Peschon, J.J., Spriggs, M.K., and Kolodkin, A.L. (2003). Semaphorin 7A promotes axon outgrowth through integrins and MAPKs. Nature 424, 398-405.

Pedraza, L., Huang, J.K., and Colman, D.R. (2001). Organizing principles of the axoglial apparatus. Neuron 30, 335-344.

Peles, E., Nativ, M., Lustig, M., Grumet, M., Schilling, J., Martinez, R., Plowman, G.D., and Schlessinger, J. (1997). Identification of a novel contactin-associated transmembrane 
receptor with multiple domains implicated in protein-protein interactions. EMBO J 16, 978-988.

Perkins, E.M., Clarkson, Y.L., Sabatier, N., Longhurst, D.M., Millward, C.P., Jack, J., Toraiwa, J., Watanabe, M., Rothstein, J.D., Lyndon, A.R., et al. (2010). Loss of beta-III spectrin leads to Purkinje cell dysfunction recapitulating the behavior and neuropathology of spinocerebellar ataxia type 5 in humans. J. Neurosci. 30, 4857-4867.

Perkins, N.D. (2007). Integrating cell-signalling pathways with NF-kappaB and IKK function. Nat. Rev. Mol. Cell Biol. 8, 49-62.

Peters, L.L., Weier, H.U., Walensky, L.D., Snyder, S.H., Parra, M., Mohandas, N., and Conboy, J.G. (1998). Four paralogous protein 4.1 genes map to distinct chromosomes in mouse and human. Genomics 54, 348-350.

Pillai, A.M., Thaxton, C., Pribisko, A.L., Cheng, J.G., Dupree, J.L., and Bhat, M.A. (2009). Spatiotemporal ablation of myelinating glia-specific neurofascin (Nfasc NF155) in mice reveals gradual loss of paranodal axoglial junctions and concomitant disorganization of axonal domains. J. Neurosci. Res. 87, 1773-1793.

Poliak, S., Gollan, L., Martinez, R., Custer, A., Einheber, S., Salzer, J.L., Trimmer, J.S., Shrager, P., and Peles, E. (1999). Caspr2, a new member of the neurexin superfamily, is localized at the juxtaparanodes of myelinated axons and associates with $\mathrm{K}+$ channels. Neuron 24, 1037-1047.

Poliak, S., Gollan, L., Salomon, D., Berglund, E.O., Ohara, R., Ranscht, B., and Peles, E. (2001). Localization of Caspr2 in myelinated nerves depends on axon-glia interactions and the generation of barriers along the axon. J. Neurosci. 21, 7568-7575.

Poliak, S., and Peles, E. (2003). The local differentiation of myelinated axons at nodes of Ranvier. Nat. Rev. Neurosci. 4, 968-980.

Poliak, S., Salomon, D., Elhanany, H., Sabanay, H., Kiernan, B., Pevny, L., Stewart, C.L., Xu, X., Chiu, S.Y., Shrager, P., et al. (2003). Juxtaparanodal clustering of Shakerlike $\mathrm{K}+$ channels in myelinated axons depends on Caspr2 and TAG-1. J. Cell Biol. 162, 1149-1160.

Porcionatto, M.A. (2006). The extracellular matrix provides directional cues for neuronal migration during cerebellar development. Brazilian J. Med. Biol. Res. 39, 313-320.

Pruss, T., Kranz, E.U., Niere, M., and Volkmer, H. (2006). A regulated switch of chick neurofascin isoforms modulates ligand recognition and neurite extension. Mol. Cell. Neurosci. 31, 354-365. 
Pruss, T., Niere, M., Kranz, E.U., and Volkmer, H. (2004). Homophilic interactions of chick neurofascin in trans are important for neurite induction. Eur. J. Neurosci. 20, 31843188.

Purves, D., Augustine, G.J., Fitzpatrick, D., Hall, W.C., LaMantia, A.-S., McNamara, J.O., and Williams, S.M. (2004). Neuroscience, 3 edn (Sunderland, MA: Sinauer Associates).

Rader, C., Stoeckli, E.T., Ziegler, U., Osterwalder, T., Kunz, B., and Sonderegger, P. (1993). Cell-cell adhesion by homophilic interaction of the neuronal recognition molecule axonin-1. Eur. J. Biochem. 215, 133-141.

Raman, I.M., and Bean, B.P. (1997). Resurgent sodium current and action potential formation in dissociated cerebellar Purkinje neurons. J. Neurosci. 17, 4517-4526.

Raman, I.M., and Bean, B.P. (1999). Properties of sodium currents and action potential firing in isolated cerebellar Purkinje neurons. Ann. N. Y. Acad. Sci. 868, 93-96.

Ramon y Cajal, S. (1894). The Croonian Lecture. La fine structure des centres nerveux. Proc. R. Soc. Lond. B. 55, 444-468

Ramon y Cajal, S. (1911). Histologie du système nerveux de l'hommes et des vertébrés. (Maloine, Paris).

Rasband, M.N. (2006). Neuron-glia interactions at the node of Ranvier. Results. Probl. Cell Differ. 43, 129-149.

Rasband, M.N. (2008). Na+ channels get anchored...with a little help. J. Cell Biol. 183, 975-977.

Rasband, M.N. (2010). The axon initial segment and the maintenance of neuronal polarity. Nat. Rev. Neurosci. 11, 552-562.

Rasband, M.N., Park, E.W., Zhen, D., Arbuckle, M.I., Poliak, S., Peles, E., Grant, S.G., and Trimmer, J.S. (2002). Clustering of neuronal potassium channels is independent of their interaction with PSD-95. J. Cell Biol. 159, 663-672.

Rasband, M.N., Tayler, J., Kaga, Y., Yang, Y., Lappe-Siefke, C., Nave, K.A., and Bansal, R. (2005). CNP is required for maintenance of axon-glia interactions at nodes of Ranvier in the CNS. Glia 50, 86-90.

Ratcliffe, C.F., Westenbroek, R.E., Curtis, R., and Catterall, W.A. (2001). Sodium channel beta1 and beta3 subunits associate with neurofascin through their extracellular immunoglobulin-like domain. J. Cell Biol. 154, 427-434. 
Rhodes, K.J., Strassle, B.W., Monaghan, M.M., Bekele-Arcuri, Z., Matos, M.F., and Trimmer, J.S. (1997). Association and colocalization of the Kvbeta1 and Kvbeta2 betasubunits with Kv1 alpha-subunits in mammalian brain $\mathrm{K}+$ channel complexes. J. Neurosci. 17, 8246-8258.

Rinaldo, L., and Hansel, C. (2010). Ataxias and cerebellar dysfunction: involvement of synaptic plasticity deficits? Funct. Neurol. 25, 135-139.

Rios, J.C., Melendez-Vasquez, C.V., Einheber, S., Lustig, M., Grumet, M., Hemperly, J., Peles, E., and Salzer, J.L. (2000). Contactin-associated protein (Caspr) and contactin form a complex that is targeted to the paranodal junctions during myelination. $J$. Neurosci. 20, 8354-8364.

Rios, J.C., Rubin, M., St Martin, M., Downey, R.T., Einheber, S., Rosenbluth, J., Levinson, S.R., Bhat, M., and Salzer, J.L. (2003). Paranodal interactions regulate expression of sodium channel subtypes and provide a diffusion barrier for the node of Ranvier. J. Neurosci. 23, 7001-7011.

Rodriguez, M., and Scheithauer, B. (1994). Ultrastructure of multiple sclerosis. Ultrastruct. Pathol. 18, 3-13.

Rolls, M.M., Satoh, D., Clyne, P.J., Henner, A.L., Uemura, T., and Doe, C.Q. (2007). Polarity and intracellular compartmentalization of Drosophila neurons. Neural Dev. 2, 7.

Roots, B.I. (2008). The phylogeny of invertebrates and the evolution of myelin. Neuron Glia Biol. 4, 101-109.

Rosenbluth, J. (1976). Intramembranous particle distribution at the node of Ranvier and adjacent axolemma in myelinated axons of the frog brain. J. Neurocytol. 5, 731-745.

Rosenbluth, J. (1981). Axoglial junctions in the mouse mutant Shiverer. Brain Res. 208, 283-297.

Rosenbluth, J. (1995). Glial membrane and axoglial junctions. In: Kettenmann H, Ranson BR , editors. Neuroglia. New York: Oxford University Press. p 613-633. (New York: Oxford University Press).

Rosenbluth, J., Dupree, J.L., and Popko, B. (2003). Nodal sodium channel domain integrity depends on the conformation of the paranodal junction, not on the presence of transverse bands. Glia 41, 318-325.

Sacco, T., De Luca, A., and Tempia, F. (2006). Properties and expression of Kv3 channels in cerebellar Purkinje cells. Mol. Cell. Neurosci. 33, 170-179.

Saito, F., Moore, S.A., Barresi, R., Henry, M.D., Messing, A., Ross-Barta, S.E., Cohn, R.D., Williamson, R.A., Sluka, K.A., Sherman, D.L., et al. (2003). Unique role of 
dystroglycan in peripheral nerve myelination, nodal structure, and sodium channel stabilization. Neuron 38, 747-758.

Sakaba, T. (2008). Two Ca(2+)-dependent steps controlling synaptic vesicle fusion and replenishment at the cerebellar basket cell terminal. Neuron 57, 406-419.

Sakisaka, T., and Takai, Y. (2004). Biology and pathology of nectins and nectin-like molecules. Curr. Opin. Cell Biol. 16, 513-521.

Salzer, J.L. (2003). Polarized domains of myelinated axons. Neuron 40, 297-318.

Salzer, J.L., Holmes, W.P., and Colman, D.R. (1987). The amino acid sequences of the myelin-associated glycoproteins: homology to the immunoglobulin gene superfamily. J. Cell Biol. 104, 957-965.

Sanchez-Ponce, D., Tapia, M., Munoz, A., and Garrido, J.J. (2008). New role of IKK alpha/beta phosphorylated I kappa B alpha in axon outgrowth and axon initial segment development. Mol. Cell. Neurosci. 37, 832-844.

Sasaki, S., and Maruyama, S. (1992). Increase in diameter of the axonal initial segment is an early change in amyotrophic lateral sclerosis. J. Neurol. Sci. 110, 114-120.

Schafer, D.P., Bansal, R., Hedstrom, K.L., Pfeiffer, S.E., and Rasband, M.N. (2004). Does paranode formation and maintenance require partitioning of neurofascin 155 into lipid rafts? J. Neurosci. 24, 3176-3185.

Schafer, D.P., Custer, A.W., Shrager, P., and Rasband, M.N. (2006). Early events in node of Ranvier formation during myelination and remyelination in the PNS. Neuron Glia Biol. 2, 69-79.

Schafer, D.P., Jha, S., Liu, F., Akella, T., McCullough, L.D., and Rasband, M.N. (2009). Disruption of the axon initial segment cytoskeleton is a new mechanism for neuronal injury. J. Neurosci. 29, 13242-13254.

Schnapp, B., Peracchia, C., and Mugnaini, E. (1976). The paranodal axo-glial junction in the central nervous system studied with thin sections and freeze-fracture. Neurosci. 1 , 181-190.

Schultz, C., Konig, H.G., Del Turco, D., Politi, C., Eckert, G.P., Ghebremedhin, E., Prehn, J.H., Kogel, D., and Deller, T. (2006). Coincident enrichment of phosphorylated IkappaBalpha, activated IKK, and phosphorylated p65 in the axon initial segment of neurons. Mol. Cell. Neurosci. 33, 68-80.

Seidl, A.H., Rubel, E.W., and Harris, D.M. (2010). Mechanisms for adjusting interaural time differences to achieve binaural coincidence detection. J. Neurosci. 30, 70-80. 
Shepherd, M.N., Pomicter, A.D., Velazco, C.S., Henderson, S.C., and Dupree, J.L. (2012). Paranodal reorganization results in the depletion of transverse bands in the aged central nervous system. Neurobiol. Aging 33, 203 e213-224.

Sherman, D.L., Tait, S., Melrose, S., Johnson, R., Zonta, B., Court, F.A., Macklin, W.B., Meek, S., Smith, A.J., Cottrell, D.F., et al. (2005). Neurofascins are required to establish axonal domains for saltatory conduction. Neuron 48, 737-742.

Shi, S.H., Jan, L.Y., and Jan, Y.N. (2003). Hippocampal neuronal polarity specified by spatially localized mPar3/mPar6 and PI 3-kinase activity. Cell 112, 63-75.

Shi, Z.T., Afzal, V., Coller, B., Patel, D., Chasis, J.A., Parra, M., Lee, G., Paszty, C., Stevens, M., Walensky, L., et al. (1999). Protein 4.1R-deficient mice are viable but have erythroid membrane skeleton abnormalities. J. Clin. Invest. 103, 331-340.

Shiga, T., Shirai, T., Grumet, M., Edelman, G.M., and Oppenheim, R.W. (1993).

Differential expression of neuron-glia cell adhesion molecule (Ng-CAM) on developing axons and growth cones of interneurons in the chick embryo spinal cord: an immunoelectron microscopic study. J. Comp. Neurol. 329, 512-518.

Shill, H.A., Adler, C.H., Sabbagh, M.N., Connor, D.J., Caviness, J.N., Hentz, J.G., and Beach, T.G. (2008). Pathologic findings in prospectively ascertained essential tremor subjects. Neurol. 70, 1452-1455.

Shingai, T., Ikeda, W., Kakunaga, S., Morimoto, K., Takekuni, K., Itoh, S., Satoh, K., Takeuchi, M., Imai, T., Monden, M., et al. (2003). Implications of nectin-like molecule2/IGSF4/RA175/SgIGSF/TSLC1/SynCAM1 in cell-cell adhesion and transmembrane protein localization in epithelial cells. J. Biol. Chem. 278, 35421-35427.

Shy, M.E. (2006). Peripheral neuropathies caused by mutations in the myelin protein zero. J. Neurol. Sci. 242, 55-66.

Simons, M., Kramer, E.M., Thiele, C., Stoffel, W., and Trotter, J. (2000). Assembly of myelin by association of proteolipid protein with cholesterol- and galactosylceramiderich membrane domains. J. Cell Biol. 151, 143-154.

Simons, M., and Trotter, J. (2007). Wrapping it up: the cell biology of myelination. Curr. Opin. Neurobiol. 17, 533-540.

Singer, C., Sanchez-Ramos, J., and Weiner, W.J. (1994). Gait abnormality in essential tremor. Mov. Disord. 9, 193-196.

Slemmer, J.E., De Zeeuw, C.I., and Weber, J.T. (2005). Don't get too excited: mechanisms of glutamate-mediated Purkinje cell death. Prog. Brain. Res. 148, 367-390. 
Smith, K.J., Blakemore, W.F., Murray, J.A., and Patterson, R.C. (1982). Internodal myelin volume and axon surface area. A relationship determining myelin thickness? J. Neurol. Sci. 55, 231-246.

Sobotzik, J.M., Sie, J.M., Politi, C., Del Turco, D., Bennett, V., Deller, T., and Schultz, C. (2009). AnkyrinG is required to maintain axo-dendritic polarity in vivo. Proc. Natl. Acad. Sci. U. S. A. 106, 17564-17569.

Somogyi, P., and Hamori, J. (1976). A quantitative electron microscopic study of the Purkinje cell axon initial segment. Neurosci. 1, 361-365.

Somogyi, P., Nunzi, M.G., Gorio, A., and Smith, A.D. (1983). A new type of specific interneuron in the monkey hippocampus forming synapses exclusively with the axon initial segments of pyramidal cells. Brain Res. 259, 137-142.

Somogyi, P., Tamas, G., Lujan, R., and Buhl, E.H. (1998). Salient features of synaptic organisation in the cerebral cortex. Brain Res. Brain Res. Rev. 26, 113-135.

Song, A.H., Wang, D., Chen, G., Li, Y., Luo, J., Duan, S., and Poo, M.M. (2009). A selective filter for cytoplasmic transport at the axon initial segment. Cell 136, 1148-1160.

Sotelo, C. (2008). Development of "Pinceaux" formations and dendritic translocation of climbing fibers during the acquisition of the balance between glutamatergic and gammaaminobutyric acidergic inputs in developing Purkinje cells. J. Comp. Neurol. 506, 240262.

Sousa, A.D., and Bhat, M.A. (2007). Cytoskeletal transition at the paranodes: the Achilles' heel of myelinated axons. Neuron Glia Biol. 3, 169-178.

Southwood, C., He, C., Garbern, J., Kamholz, J., Arroyo, E., and Gow, A. (2004). CNS myelin paranodes require Nkx6-2 homeoprotein transcriptional activity for normal structure. J. Neurosci. 24, 11215-11225.

Stokin, G.B., Lillo, C., Falzone, T.L., Brusch, R.G., Rockenstein, E., Mount, S.L., Raman, R., Davies, P., Masliah, E., Williams, D.S., et al. (2005). Axonopathy and transport deficits early in the pathogenesis of Alzheimer's disease. Science 307, 12821288.

Stolze, H., Petersen, G., Raethjen, J., Wenzelburger, R., and Deuschl, G. (2001). The gait disorder of advanced essential tremor. Brain 124, 2278-2286.

Sudarov, A., Turnbull, R.K., Kim, E.J., Lebel-Potter, M., Guillemot, F., and Joyner, A.L. (2011). Ascl1 genetics reveals insights into cerebellum local circuit assembly. J. Neurosci. 31, 11055-11069. 
Sun, C.X., Robb, V.A., and Gutmann, D.H. (2002). Protein 4.1 tumor suppressors: getting a FERM grip on growth regulation. J. Cell Sci. 115, 3991-4000.

Susuki, K., Raphael, A.R., Ogawa, Y., Stankewich, M.C., Peles, E., Talbot, W.S., and Rasband, M.N. (2011). Schwann cell spectrins modulate peripheral nerve myelination. Proc. Natl. Acad. Sci. U. S. A. 108, 8009-8014.

Susuki, K., and Rasband, M.N. (2008). Molecular mechanisms of node of Ranvier formation. Curr. Opin. Cell Biol. 20, 616-623.

Susuki, K., Rasband, M.N., Tohyama, K., Koibuchi, K., Okamoto, S., Funakoshi, K., Hirata, K., Baba, H., and Yuki, N. (2007). Anti-GM1 antibodies cause complementmediated disruption of sodium channel clusters in peripheral motor nerve fibers. J. Neurosci. 27, 3956-3967.

Sutherland, M.L., Williams, S.H., Abedi, R., Overbeek, P.A., Pfaffinger, P.J., and Noebels, J.L. (1999). Overexpression of a Shaker-type potassium channel in mammalian central nervous system dysregulates native potassium channel gene expression. Proc. Natl. Acad. Sci. U. S. A. 96, 2451-2455.

Tait, S., Gunn-Moore, F., Collinson, J.M., Huang, J., Lubetzki, C., Pedraza, L., Sherman, D.L., Colman, D.R., and Brophy, P.J. (2000). An oligodendrocyte cell adhesion molecule at the site of assembly of the paranodal axo-glial junction. J. Cell Biol. 150, 657-666.

Takai, Y., Irie, K., Shimizu, K., Sakisaka, T., and Ikeda, W. (2003). Nectins and nectinlike molecules: roles in cell adhesion, migration, and polarization. Cancer Sci. 94, 655667.

Tao-Cheng, J.H., and Rosenbluth, J. (1983). Axolemmal differentiation in myelinated fibers of rat peripheral nerves. Brain Res. 285, 251-263.

Tasaki, I. (1959). Physiologic properties of the myelin sheath and of the node of Ranvier. Prog. Neurobiol. 4, 159-172.

Thaxton, C., and Bhat, M.A. (2009). Myelination and regional domain differentiation of the axon. Results Probl. Cell Differ. 48, 1-28.

Thaxton, C., Pillai, A.M., Pribisko, A.L., Dupree, J.L., and Bhat, M.A. (2011). Nodes of Ranvier act as barriers to restrict invasion of flanking paranodal domains in myelinated axons. Neuron 69, 244-257.

Thaxton, C., Pillai, A.M., Pribisko, A.L., Labasque, M., Dupree, J.L., Faivre-Sarrailh, C., and Bhat, M.A. (2010). In vivo deletion of immunoglobulin domains 5 and 6 in neurofascin (Nfasc) reveals domain-specific requirements in myelinated axons. J. Neurosci. 30, 4868-4876. 
Traka, M., Dupree, J.L., Popko, B., and Karagogeos, D. (2002). The neuronal adhesion protein TAG-1 is expressed by Schwann cells and oligodendrocytes and is localized to the juxtaparanodal region of myelinated fibers. J. Neurosci. 22, 3016-3024.

Traka, M., Goutebroze, L., Denisenko, N., Bessa, M., Nifli, A., Havaki, S., Iwakura, Y., Fukamauchi, F., Watanabe, K., Soliven, B., et al. (2003). Association of TAG-1 with Caspr2 is essential for the molecular organization of juxtaparanodal regions of myelinated fibers. J. Cell Biol. 162, 1161-1172.

Trapp, B.D. (1990). Myelin-associated glycoprotein. Location and potential functions. Ann. N. Y. Acad. Sci. 605, 29-43.

Trapp, B.D., Andrews, S.B., Wong, A., O'Connell, M., and Griffin, J.W. (1989). Colocalization of the myelin-associated glycoprotein and the microfilament components, Factin and spectrin, in Schwann cells of myelinated nerve fibres. J. Neurocytol. 18, 47-60.

Trapp, B.D., and Nave, K.A. (2008). Multiple sclerosis: an immune or neurodegenerative disorder? Annu. Rev. Neurosci. 31, 247-269.

Trapp, B.D., and Quarles, R.H. (1984). Immunocytochemical localization of the myelinassociated glycoprotein. Fact or artifact? J. Neuroimmunol. 6, 231-249.

Trapp, B.D., Quarles, R.H., and Suzuki, K. (1984). Immunocytochemical studies of quaking mice support a role for the myelin-associated glycoprotein in forming and maintaining the periaxonal space and periaxonal cytoplasmic collar of myelinating Schwann cells. J. Cell Biol. 99, 594-606.

Ushkaryov, Y.A., Petrenko, A.G., Geppert, M., and Sudhof, T.C. (1992). Neurexins: synaptic cell surface proteins related to the alpha-latrotoxin receptor and laminin. Science 257, 50-56.

Van Wart, A., and Matthews, G. (2006). Impaired firing and cell-specific compensation in neurons lacking nav1.6 sodium channels. J. Neurosci. 26, 7172-7180.

Volk, D.W., and Lewis, D.A. (2002). Impaired prefrontal inhibition in schizophrenia: relevance for cognitive dysfunction. Physiol. Behav. 77, 501-505.

Volkmer, H., Hassel, B., Wolff, J.M., Frank, R., and Rathjen, F.G. (1992). Structure of the axonal surface recognition molecule neurofascin and its relationship to a neural subgroup of the immunoglobulin superfamily. J. Cell Biol. 118, 149-161.

Wallace, R.H., Marini, C., Petrou, S., Harkin, L.A., Bowser, D.N., Panchal, R.G., Williams, D.A., Sutherland, G.R., Mulley, J.C., Scheffer, I.E., et al. (2001). Mutant GABA(A) receptor gamma2-subunit in childhood absence epilepsy and febrile seizures. Nat. Genet. 28, 49-52. 
Walsh, F.S., and Doherty, P. (1997). Neural cell adhesion molecules of the immunoglobulin superfamily: role in axon growth and guidance. Annu. Rev. Cell Dev. Biol. 13, 425-456.

Wang, H., Kunkel, D.D., Martin, T.M., Schwartzkroin, P.A., and Tempel, B.L. (1993). Heteromultimeric $\mathrm{K}+$ channels in terminal and juxtaparanodal regions of neurons. Nature $365,75-79$.

Wang, V.Y., and Zoghbi, H.Y. (2001). Genetic regulation of cerebellar development. Nat. Rev. Neurosci. 2, 484-491.

Wang, Z., Gerstein, M., and Snyder, M. (2009). RNA-Seq: a revolutionary tool for transcriptomics. Nat. Rev. Genet. 10, 57-63.

Ward, R.E.t., Lamb, R.S., and Fehon, R.G. (1998). A conserved functional domain of Drosophila coracle is required for localization at the septate junction and has membraneorganizing activity. J. Cell Biol. 140, 1463-1473.

Watanabe, H., Nagata, E., Kosakai, A., Nakamura, M., Yokoyama, M., Tanaka, K., and Sasai, H. (2000). Disruption of the epilepsy KCNQ2 gene results in neural hyperexcitability. J. Neurochem. 75, 28-33.

Waters, M.F., Minassian, N.A., Stevanin, G., Figueroa, K.P., Bannister, J.P., Nolte, D., Mock, A.F., Evidente, V.G., Fee, D.B., Muller, U., et al. (2006). Mutations in voltagegated potassium channel KCNC3 cause degenerative and developmental central nervous system phenotypes. Nat. Genet. 38, 447-451.

Waxman, S.G., and Ritchie, J.M. (1993). Molecular dissection of the myelinated axon. Ann. Neurol. 33, 121-136.

Wiencken-Barger, A.E., Mavity-Hudson, J., Bartsch, U., Schachner, M., and Casagrande, V.A. (2004). The role of L1 in axon pathfinding and fasciculation. Cereb. Cortex 14, 121131.

Winckler, B., Forscher, P., and Mellman, I. (1999). A diffusion barrier maintains distribution of membrane proteins in polarized neurons. Nature 397, 698-701.

Wolswijk, G., and Balesar, R. (2003). Changes in the expression and localization of the paranodal protein Caspr on axons in chronic multiple sclerosis. Brain 126, 1638-1649.

Wood, R.L. (1959). Intercellular attachment in the epithelium of Hydra as revealed by electron microscopy. J. Biophys. Biochem. Cytol. 6, 343-352.

Wooten, M.W., Seibenhener, M.L., Neidigh, K.B., and Vandenplas, M.L. (2000). Mapping of atypical protein kinase $\mathrm{C}$ within the nerve growth factor signaling cascade: relationship to differentiation and survival of PC12 cells. Mol. Cell. Biol. 20, 4494-4504. 
Xie, G., Harrison, J., Clapcote, S.J., Huang, Y., Zhang, J.Y., Wang, L.Y., and Roder, J.C. (2010). A new Kv1.2 channelopathy underlying cerebellar ataxia. J. Biol. Chem. 285, 32160-32173.

Yageta, M., Kuramochi, M., Masuda, M., Fukami, T., Fukuhara, H., Maruyama, T., Shibuya, M., and Murakami, Y. (2002). Direct association of TSLC1 and DAL-1, two distinct tumor suppressor proteins in lung cancer. Cancer Res. 62, 5129-5133.

Yamakawa, H., Ohara, R., Nakajima, D., Nakayama, M., and Ohara, O. (1999). Molecular characterization of a new member of the protein 4.1 family (brain 4.1) in rat brain. Brain Res. Mol. Brain Res. 70, 197-209.

Yang, Y., Lacas-Gervais, S., Morest, D.K., Solimena, M., and Rasband, M.N. (2004). BetaIV spectrins are essential for membrane stability and the molecular organization of nodes of Ranvier. J. Neurosci. 24, 7230-7240.

Yi, C., McCarty, J.H., Troutman, S.A., Eckman, M.S., Bronson, R.T., and Kissil, J.L. (2005). Loss of the putative tumor suppressor band 4.1B/Dall gene is dispensable for normal development and does not predispose to cancer. Mol. Cell. Biol. 25, 1005210059.

Yoshimura, T., Arimura, N., and Kaibuchi, K. (2006a). Signaling networks in neuronal polarization. J. Neurosci. 26, 10626-10630.

Yoshimura, T., Arimura, N., Kawano, Y., Kawabata, S., Wang, S., and Kaibuchi, K. (2006b). Ras regulates neuronal polarity via the PI3-kinase/Akt/GSK-3beta/CRMP-2 pathway. Biochem. Biophys. Res. Commun. 340, 62-68.

Yu, R.C., and Bunge, R.P. (1975). Damage and repair of the peripheral myelin sheath and node of Ranvier after treatment with trypsin. J. Cell Biol. 64, 1-14.

Zagha, E., Lang, E.J., and Rudy, B. (2008). Kv3.3 channels at the Purkinje cell soma are necessary for generation of the classical complex spike waveform. J. Neurosci. 28, 12911300 .

Zhang, L., and Goldman, J.E. (1996). Generation of cerebellar interneurons from dividing progenitors in white matter. Neuron 16, 47-54.

Zhang, X., and Bennett, V. (1998). Restriction of 480/270-kD ankyrin G to axon proximal segments requires multiple ankyrin G-specific domains. J. Cell Biol. 142, 15711581.

Zhang, X., Davis, J.Q., Carpenter, S., and Bennett, V. (1998). Structural requirements for association of neurofascin with ankyrin. J. Biol. Chem. 273, 30785-30794. 
Zhang, Y., Bekku, Y., Dzhashiashvili, Y., Armenti, S., Meng, X., Sasaki, Y., Milbrandt, J., and Salzer, J.L. (2012). Assembly and maintenance of nodes of ranvier rely on distinct sources of proteins and targeting mechanisms. Neuron 73, 92-107.

Zhou, D., Lambert, S., Malen, P.L., Carpenter, S., Boland, L.M., and Bennett, V. (1998). AnkyrinG is required for clustering of voltage-gated $\mathrm{Na}$ channels at axon initial segments and for normal action potential firing. J. Cell Biol. 143, 1295-1304.

Zhuchenko, O., Bailey, J., Bonnen, P., Ashizawa, T., Stockton, D.W., Amos, C., Dobyns, W.B., Subramony, S.H., Zoghbi, H.Y., and Lee, C.C. (1997). Autosomal dominant cerebellar ataxia (SCA6) associated with small polyglutamine expansions in the alpha 1A-voltage-dependent calcium channel. Nat. Genet. 15, 62-69.

Zoghbi, H.Y. (1995). Spinocerebellar ataxia type 1. Clin. Neurosci. 3, 5-11.

Zoghbi, H.Y. (2000). Spinocerebellar ataxias. Neurobiol. Dis. 7, 523-527.

Zoghbi, H.Y., and Orr, H.T. (2009). Pathogenic mechanisms of a polyglutaminemediated neurodegenerative disease, spinocerebellar ataxia type 1. J. Biol. Chem. 284, 7425-7429.

Zonta, B., Desmazieres, A., Rinaldi, A., Tait, S., Sherman, D.L., Nolan, M.F., and Brophy, P.J. (2011). A critical role for Neurofascin in regulating action potential initiation through maintenance of the axon initial segment. Neuron 69, 945-956.

Zonta, B., Tait, S., Melrose, S., Anderson, H., Harroch, S., Higginson, J., Sherman, D.L., and Brophy, P.J. (2008). Glial and neuronal isoforms of Neurofascin have distinct roles in the assembly of nodes of Ranvier in the central nervous system. J. Cell Biol. 181, 1169-1177. 